University of CALIFornia

Los Angeles

\title{
Search for R-Parity Violating Supersymmetry in the Multilepton Final State
}

\author{
A dissertation submitted in partial satisfaction \\ of the requirements for the degree \\ Doctor of Philosophy in Physics
}

by

Alon Jacques Attal 
(C) Copyright by

Alon Jacques Attal

2006 
The dissertation of Alon Jacques Attal is approved.

$\begin{array}{r}\text { Mark Moldwin } \\ \hline \text { Katsushi Arisaka } \\ \hline \text { Ben Nefkens } \\ \hline \text { Jay Hauser, Committee Chair }\end{array}$

University of California, Los Angeles

2006 
To my parents. 


\section{Table of Contents}

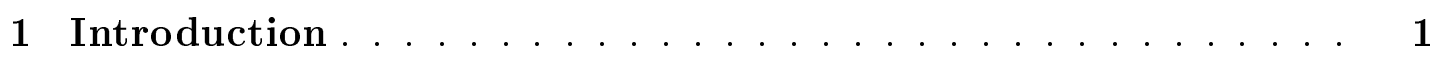

2 Theory \& Motivation ................. 5

2.1 What is a particle? .................. 5

2.2 Spin \& the Pauli Exclusion Principle . . . . . . . . . . 6

2.3 Standard Model of Particle Physics . . . . . . . . . . . 7

2.4 Asymptotic Freedom \& Color Confinement . . . . . . . . . . . 9

2.5 Particle Interactions . . . . . . . . . . . . 10

2.6 Proton Antiproton Collisions . . . . . . . . . . . . 11

2.7 Symmetry ............................... 13

2.8 Higgs Field ... . . . . . . . . . . . 15

2.9 Spontaneous Symmetry Breaking (Higgs Mechanism) . . . . . . . 17

2.10 Motivation for Physics Beyond the Standard Model . . . . . . . . 19

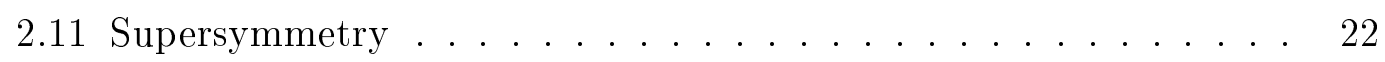

2.11.1 Supersymmetry Breaking . . . . . . . . . . 23

2.11.2 Supersymmetric Particle Spectrum . . . . . . . . . 24

2.11 .3 Minimal Supergravity . . . . . . . . . . 26

2.11 .4 R-parity ........................ 28

2.11 .5 R-parity Violation ................. 29

2.11.6 4-Lepton Signature . . . . . . . . . . . . . 30

2.11.7 R-parity Violating Supersymmetry . . . . . . . . . 30 
2.11.8 Dependence on Minimal Supergravity Parameters . . . . . 33

2.12 Other Theories Resulting in 4 or More Leptons . . . . . . . . 37

2.12.1 Doubly-Charged Higgs Pair Production . . . . . . . . . 37

2.12.2 Non-Minimal Supersymmetric Models . . . . . . . . . . . 37

3 Fermilab Tevatron \& CDF Detector . . . . . . . . . . . 42

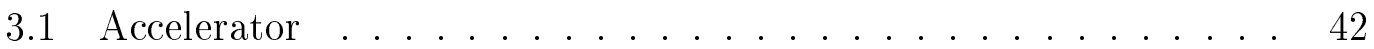

3.2 CDF Coordinate System . . . . . . . . . . . . 47

3.3 CDF Detector ....................... 47

3.3.1 Cerenkov Luminosity Counter . . . . . . . . . . . 48

3.3.2 Tracking Systems . . . . . . . . . . . . . 51

3.3.3 Calorimeters ................. 54

3.3.4 Muon Detectors ................ 58

3.4 Event Triggers and Data Acquisition . . . . . . . . . 60

3.4.1 The Shift Crew . . . . . . . . . . . . 61

3.4.2 Good Run Requirements . . . . . . . . . . . 61

3.4.3 CDF Data Reconstruction Software . . . . . . . . . 64

3.4.4 Event Simulation ............... 66

4 Particle Identification . . . . . . . . . . . . . . 68

4.1 Electron Identification . . . . . . . . . . . 69

4.2 Muon Identification . . . . . . . . . . . . . . . . 70

4.3 Lepton Identification Cuts . . . . . . . . . . . . 71

4.3.1 Electron Identification Cuts ........... 72 
4.3.2 Muon Identification Cuts ............ 75

4.4 Lepton Identification Efficiencies . . . . . . . . . . . . 76

4.5 Jets Misidentified as Leptons . . . . . . . . . . . . 79

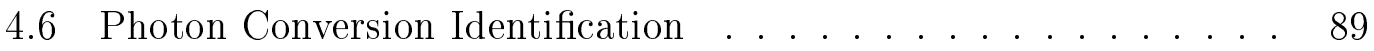

4.7 Cosmic Ray Muon Identification . . . . . . . . . . . . . . . . 90

5 Simulated and CDF Run II Data Collections . . . . . . . . . . 92

5.1 R-Parity Violating Supersymmetric Event Simulation . . . . . . . 92

5.2 Data Collection ...................... 94

5.3 Background MC Samples . . . . . . . . . . . . . 95

5.3.1 Drell-Yan Background ............ 96

5.3.2 Backgrounds From Misidentified Jets . . . . . . . . . 97

5.3.3 Backgrounds From Photon Conversions . . . . . . . . 97

5.3.4 Diboson Backgrounds .............. 98

5.3.5 Top Quark Background .............. 99

5.3.6 Heavy Flavor Backgrounds . . . . . . . . . . . . . 99

5.4 Background and Signal Predictions . . . . . . . . . . . 99

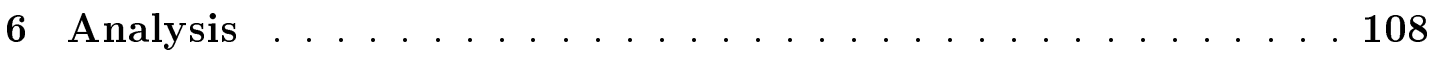

6.1 Event Selection . . . . . . . . . . . . 109

6.2 Signal Samples . . . . . . . . . . . . . . 110

6.3 Validation ........................... 112

6.4 Uncertainties ........................ 114

6.4.1 Statistical Uncertainty .............. 114 
6.4.2 Systematic Uncertainty.............. 118

7 Results . . . . . . . . . . . . . . . . . 132

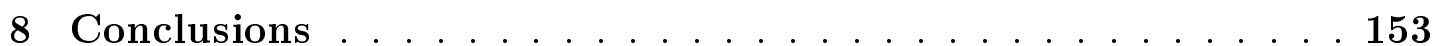

A Almost Discovering Supersymmetry ............. 154

A.1 Introduction . . . . . . . . . . . . . . 154

A.2 Discovery? . . . . . . . . . . . . . . 156

A.3 The $\Delta \phi$ Control Sample . . . . . . . . . . . . . . . . . 157

A.4 The Trilepton Invariant Mass Requirement . . . . . . . . . . . . . 158

A.5 $Z / \gamma^{*}+\gamma$ Background Prediction . . . . . . . . . 159

A.6 Electron Impact Parameter Cut . . . . . . . . . . . . . . 162

A.7 Conclusions . . . . . . . . . . . . . . . 162

B Conversion Removal Efficiency . . . . . . . . . . . . . 164

B.1 Introduction . . . . . . . . . . . . . . . . . . 164

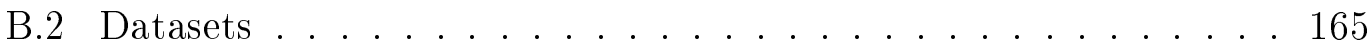

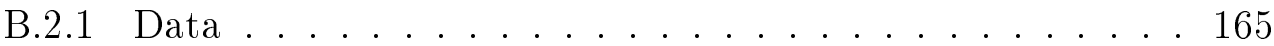

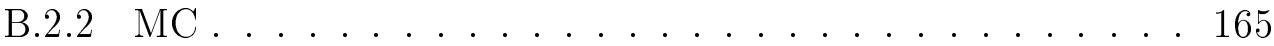

B.3 Photon Conversion Selection _. . . . . . . . . . . 165

B.4 Background .......................... 168

B.4.1 Background Subtraction ........... 176

B.5 Efficiency Measurement . . . . . . . . . . . . 178

B.6 Scale Factor Measurement and Implementation _ . . . . . . . . 183 
B.7 Uncertainty . . . . . . . . . . . . . . . . . . . . 184

B.7.1 $p_{T}$ Threshold . . . . . . . . . . . . . . . 185

B.7.2 $\Delta Z$ Cut $\ldots \ldots \ldots \ldots \ldots \ldots \ldots \ldots$

B.7.3 Background Subtraction . . . . . . . . . . . . 185

B.7.4 CES Reconstruction Efficiency . . . . . . . . . . . 185

B.7.5 Eta Dependence . . . . . . . . . . . . . . . 186

B.7.6 Total Uncertainty . . . . . . . . . . . . . . . 186

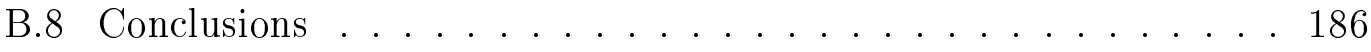

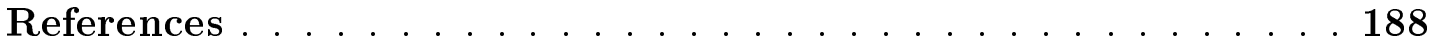




\section{List of Figures}

2.1 Definition of helicity, where $\hat{v}$ is the particle direction and $\hat{s}$ is the component of spin along $\hat{v} \ldots \ldots \ldots 7$

2.26 -fold symmetry of a snowflake . . . . . . . . . . 15

2.3 One dimensional scalar field $(\phi)$ potential. . . . . . . . . 18

2.4 Strength of each SM force $\left(\alpha_{i}\right)$ as a function of energy scale $(\mu) . \quad$. 22

2.5 Cancellation of divergent corrections to the Higgs mass. . . . . . . 23

2.6 Strength of each SM force $\left(\alpha_{i}\right)$ as a function of energy scale $(\mu)$ after including supersymmetric particles. . . . . . . . . 26

2.7 Superpartner masses for a particular choice of mSUGRA parameters. 28

2.8 Muon decay through a) the weak force and b) via an $\not R_{p}$ violating vertex. Muon decay is also possible with $\lambda_{122}$ by replacing the $\tilde{e}$ with a $\tilde{\mu}$ in the second diagram. . . . . . . . . . . 32

2.9 Chargino-neutralino and chargino pair production in proton-antiproton

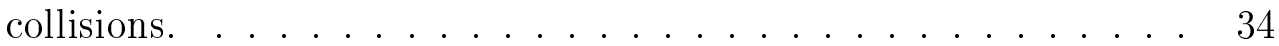

2.10 "Cascade" decays of neutralinos (top) and charginos (bottom). . . 35

2.11 Two possible Feynman diagrams for $\not R_{p}$ SUSY decay of the LSP via each of a) the $\lambda_{121}$ coupling, and b) the $\lambda_{122}$ coupling. . . . . . 36

2.12 Mass of the lightest neutralino as a function of $M_{1 / 2}$ for both signs of the higgsino mass parameter $\mu, M_{0}=250 \mathrm{GeV} / c^{2}, \tan \beta=5$, and $A_{0}=0 \ldots \ldots \ldots \ldots \ldots \ldots$ 
2.13 Mass of the lightest neutralino as a function of $\tan \beta$ for both signs of the higgsino mass parameter $\mu, M_{0}=250 \mathrm{GeV} / c^{2}, M_{1 / 2}=260$ $\mathrm{GeV} / c^{2}$, and $A_{0}=0 \ldots \ldots \ldots \ldots$

2.14 Mass of the lightest neutralino as a function of $M_{0}$ for both signs of the higgsino mass parameter $\mu, M_{1 / 2}=260 \mathrm{GeV} / c^{2}, \tan \beta=5$, and $A_{0}=0 \ldots \ldots \ldots \ldots \ldots$

2.15 Mass of the lightest neutralino as a function of $\tan \beta$ for both signs of the higgsino mass parameter $\mu, M_{0}=250 \mathrm{GeV} / c^{2}, M_{1 / 2}=260$ $\mathrm{GeV} / c^{2}$, and $\tan \beta=5 \ldots \ldots \ldots \ldots$

2.16 LSP mass as a function of $\mathrm{M}_{1 / 2}, \mathrm{M}_{0}$ (top), and $\tan \beta$ (bottom). The mass is highly dependent on $\mathrm{M}_{1 / 2}$ but has very little dependence on $\mathrm{M}_{0}$ or $\tan \beta$. The following parameters are used: $M_{0}=250$ $\mathrm{GeV} / c^{2}, \tan \beta=5, \mu>0$, and $A_{0}=0 \ldots \ldots$

3.1 Fermilab Tevatron accelerator chain. . . . . . . . . . .

3.2 Integrated luminosity collected by the CDF detector for Run 2 . The top line corresponds to luminosity which is produced at the Tevatron and the bottom line corresponds to luminosity where the collision events are successfully recorded by CDF and available for

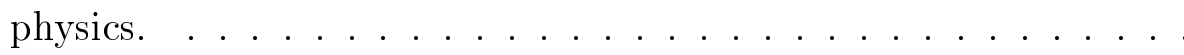

3.3 Schematic view of the northwest quadrant of the CDF detector. . 
3.4 Cerenkov Luminosity Counter (CLC) consisting of 3 layers of Cerenkov counters. The middle figure shows a cross section of the Cerenkov counters when looking from the side. The upper figure zooms in on the far end of the counters followed by the photomultiplier tubes. The bottom figure shows a cross section of the CLC when looking from the direction of the beam pipe. . . . . . . . . 50

3.5 Schematic of one quadrant of the CDF tracking system. The inner part consists of a silicon microstrip detector including Layer 00 (not shown), Silicon Vertex Detector (SVX), and Intermediate Silicon Layers (ISL). The outer part consists of the Central Outer Tracker

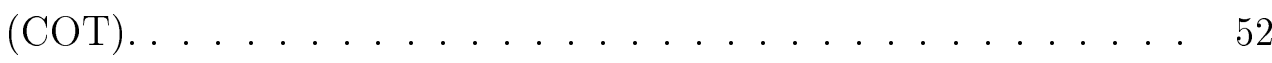

3.6 Silicon tracking system. On the left is a cross section of the silicon detectors when looking in the direction of the beam pipe. On the right is an $r-z$ view of the silicon detectors, not drawn to scale. $\quad 54$

3.7 Schematic of a single COT cell within superlayer $2 \ldots \ldots \ldots \ldots$

3.8 Schematic of one quadrant of the plug calorimeter including the EM calorimeter, hadronic calorimeter, and shower maximum detector, labeled "position detector," embedded in the EM section. The plug calorimeter covers $1.1<|\eta|<3.6$ and has the full $2 \pi \phi$

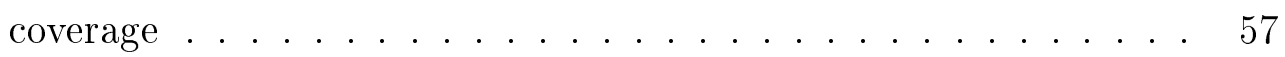

3.9 Strip layout within one wedge of the plug shower maximum (PES) detector. . . . . . . . . . . . . . . . 58

3.10 Location of the muon detectors in the $\eta$ - $\phi$ plane. . . . . . . 59

3.11 Block diagram depicting the CDF trigger system and path of online data culminating in storage on tapes. . . . . . . . . . . 62 
3.12 Block diagram of the CDF event builder in which the raw data from the front-end electronics is collected and sent to the level 3

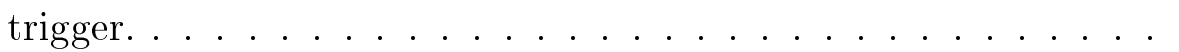

4.1 Invariant mass distribution for $Z \rightarrow \mu^{+} \mu^{-}$events peaked at the $Z$ boson mass of $91 \mathrm{GeV} / c^{2} \ldots \ldots \ldots \ldots$

4.2 Probability for a CEM cluster with $E_{T}>4 \mathrm{GeV}$ to be misidentified as a loose central electron (LCE), as measured in four samples with high concentrations of jets. . . . . . . . . . . 82

4.3 Probability for a CEM cluster with $E_{T}>4 \mathrm{GeV}$ to be misidentified as a tight central electron (TCE), as measured in four samples with high concentrations of jets. . . . . . . . . . . .

4.4 Probability for a PEM cluster with $E_{T}>8 \mathrm{GeV}$ to be misidentified as a stand-alone plug electron with $1.1<|\eta|<1.3$, as measured in four samples with high concentrations of jets. . . . . . .

4.5 Probability for a PEM cluster with $E_{T}>8 \mathrm{GeV}$ to be misidentified as a stand-alone plug electron with $|\eta|>1.3$, as measured in four samples with high concentrations of jets. . . . . . . . . .

4.6 Probability for a PEM cluster with $E_{T}>15 \mathrm{GeV}$ to be misidentified as a phoenix electron, as measured in four samples with high concentrations of jets. . . . . . . . . . . . 86

4.7 Probability for an isolated track with $p_{T}>4 \mathrm{GeV} / c$ to be misidentified as a CMUP muon, as measured in four samples with high concentrations of jets. ................. 
4.8 Probability for an isolated track with $p_{T}>4 \mathrm{GeV} / c$ to be misidentified as a CMX muon, as measured in four samples with high concentrations of jets. . . . . . . . . . . . 88

4.9 Probability for an isolated track with $p_{T}>10 \mathrm{GeV} / c$ to be misidentified as a CMIO muon, as measured in four samples with high concentrations of jets. . . . . . . . . . . . .

4.10 Photon conversion $\left(\gamma \rightarrow e^{+} e^{-}\right)$in the presence of an atomic nucleus $(\mathrm{Z}) \ldots \ldots \ldots \ldots \ldots \ldots \ldots \ldots$

5.1 Generated $\mathrm{p}_{T}$ distribution of the four leptons from the $\tilde{\chi}_{1}^{0}$ decays for $\tilde{\chi}_{1}^{0}$ mass $=99.4 \mathrm{GeV} / c^{2}$. In each event, the leptons are ordered in $p_{T}$ with largest plotted on top and smallest plotted on bottom. The mean of the distributions are: 45, 32, 21, and $12 \mathrm{GeV} / c . \ldots 103$

5.2 Generated $\eta$ distribution of the four leptons from the $\tilde{\chi}_{1}^{0}$ decays for $\tilde{\chi}_{1}^{0}$ mass $=99.4 \mathrm{GeV} / c^{2}$. In each event, the leptons are ordered in $p_{T}$ with largest plotted on top and smallest plotted on bottom. . . 104

6.1 Background contributions as a function of the number of reconstructed leptons in each event before the event selection cuts are applied. Each event must have at least one high- $p_{T}$ TCE, CMUP, or CMX lepton. The signal $\left(M_{0}=250, M_{1 / 2}=260, \tan \beta=5\right.$, $A_{0}=0$, and $\left.\mu>0\right)$ and background samples are normalized to $346 \mathrm{pb}^{-1}$

6.2 Invariant mass, and $\mathrm{p}_{T}$ distributions for the events within the trilepton control samples. The $\mathbb{R}_{p}$ SUSY signal $\left(M_{0}=250\right.$, $M_{1 / 2}=260, \tan \beta=5, A_{0}=0$, and $\left.\mu>0\right)$ is stacked on top of the backgrounds. . . . . . . . . . . . . . . . 124 
6.3 2nd and 3rd lepton $\mathrm{p}_{T}$ distributions for the events within the trilepton control samples. The $\not R_{p}$ SUSY signal $\left(M_{0}=250, M_{1 / 2}=260\right.$, $\tan \beta=5, A_{0}=0$, and $\left.\mu>0\right)$ is stacked on top of the backgrounds. 125

6.4 $\Delta \phi$ (top plot) and trilepton invariant mass (bottom plot) distributions for the events within the trilepton control samples. The $\not R$ SUSY signal $\left(M_{0}=250, M_{1 / 2}=260, \tan \beta=5, A_{0}=0\right.$, and $\mu>0$ ) is stacked on top of the backgrounds. . . . . . . . 126

6.5 Missing transverse energy distribution for the events within the trilepton control samples. The $\not R_{p}$ SUSY signal $\left(M_{0}=250, M_{1 / 2}=\right.$ $260, \tan \beta=5, A_{0}=0$, and $\mu>0$ ) is multiplied by a factor of 10 for easier visibility and plotted independently of the backgrounds.

6.6 Scatter plot of the predicted background events ( $y$ axis) versus the number of observed events in data ( $x$ axis) for each control sample. The points correspond to the control samples while the vertical error bars corresponds to the combined uncertainty on the data and MC. Due to lack of statistics, the trilepton control samples are not broken down by lepton type. The line is a visual guide signifying perfect agreement between data and MC. . . . . 128

6.7 Agreement between data and simulation for ee and $\mu \mu$ control samples. . . . . . . . . . . . . . . . . 129

6.8 Agreement between data and simulation in $\mathrm{e} \mu$ and $\geq 3$-lepton control samples. . . . . . . . . . . . . . . 130 
7.1 Combined invariant mass of the three identified leptons for the events within the trilepton signal samples. The $\not_{p}$ SUSY signal $\left(M_{0}=250, M_{1 / 2}=260, \tan \beta=5, A_{0}=0\right.$, and $\left.\mu>0\right)$ is stacked on top of the backgrounds. . . . . . . . . . . . . . 135

7.2 Event displays for trilepton signal event Run=163009, Event $=110391$. The top plot shows a cross section of the CDF detector including all tracks above $0.5 \mathrm{GeV} / c$ and calorimeter energy within the EM (purple) and the hadronic (blue) sections. The bottom plot displays the energy within each calorimeter cluster in the $\eta-\phi$ plane. The grid represents the calorimeter towers. . . . . . . . . . 140

7.3 Missing transverse energy distribution of the events within the trilepton signal samples. The $\not R_{p}$ SUSY signal $\left(M_{0}=250, M_{1 / 2}=\right.$ $260, \tan \beta=5, A_{0}=0$, and $\mu>0$ ) is plotted independently of the backgrounds. . . . . . . . . . . . . . . . . 141

7.4 Invariant mass of the first two leptons (top) and 1st lepton $p_{T}$ (bottom) distributions for the events within the trilepton signal samples. The $R_{p}$ SUSY signal $\left(M_{0}=250, M_{1 / 2}=260, \tan \beta=5\right.$, $A_{0}=0$, and $\left.\mu>0\right)$ is stacked on top of the backgrounds. . . 142

7.5 2nd lepton $\mathrm{p}_{T}$ (top) and 3rd lepton $\mathrm{p}_{T}$ (bottom) distributions for the events within the trilepton signal samples. The $\not R_{p}$ SUSY signal $\left(M_{0}=250, M_{1 / 2}=260, \tan \beta=5, A_{0}=0\right.$, and $\left.\mu>0\right)$ is stacked on top of the backgrounds. . . . . . . . . . . . . 143

7.6 $\Delta \phi$ of the first two leptons (top) and sum of the lepton $\mathrm{p}_{T}$ (bottom) distributions for the events within the trilepton signal samples. The $\not R_{p}$ SUSY signal $\left(M_{0}=250, M_{1 / 2}=260, \tan \beta=5, A_{0}=0\right.$, and $\mu>0)$ is stacked on top of the backgrounds. . . . . . 144 
7.7 $\mathbb{R}_{p}$ SUSY mass lower limits for $\lambda_{121}$ and $\mu>0$. Expected limits: $\mathrm{M}\left(\tilde{\chi}_{1}^{0}\right)>99.8 \mathrm{GeV} / c^{2}, \mathrm{M}\left(\tilde{\chi}_{1}^{ \pm}\right)>182.1 \mathrm{GeV} / c^{2}$. Observed limits: $\mathrm{M}\left(\tilde{\chi}_{1}^{0}\right)>94.8 \mathrm{GeV} / c^{2}, \mathrm{M}\left(\tilde{\chi}_{1}^{ \pm}\right)>172.6 \mathrm{GeV} / c^{2} \ldots \ldots \ldots 148$

$7.8 \not R_{p}$ SUSY mass lower limits for $\lambda_{121}$ and $\mu<0$. Expected limits: $\mathrm{M}\left(\tilde{\chi}_{1}^{0}\right)>95.5 \mathrm{GeV} / c^{2}, \mathrm{M}\left(\tilde{\chi}_{1}^{ \pm}\right)>181.3 \mathrm{GeV} / c^{2}$. Observed limits: $\mathrm{M}\left(\tilde{\chi}_{1}^{0}\right)>88.4 \mathrm{GeV} / c^{2}, \mathrm{M}\left(\tilde{\chi}_{1}^{ \pm}\right)>167.4 \mathrm{GeV} / c^{2} \ldots \ldots \ldots 149$

$7.9 \quad \not R_{p}$ SUSY mass lower limits for $\lambda_{122}$ and $\mu>0$. Expected limits: $\mathrm{M}\left(\tilde{\chi}_{1}^{0}\right)>103.6 \mathrm{GeV} / c^{2}, \mathrm{M}\left(\tilde{\chi}_{1}^{ \pm}\right)>189.2 \mathrm{GeV} / c^{2}$. Observed limits: $\mathrm{M}\left(\tilde{\chi}_{1}^{0}\right)>108.4 \mathrm{GeV} / c^{2}, \mathrm{M}\left(\tilde{\chi}_{1}^{ \pm}\right)>198.7 \mathrm{GeV} / c^{2} . \ldots \ldots . . \ldots 150$

$7.10 \not R_{p}$ SUSY mass lower limits for $\lambda_{122}$ and $\mu<0$. Expected limits: $\mathrm{M}\left(\tilde{\chi}_{1}^{0}\right)>100.2 \mathrm{GeV} / c^{2}, \mathrm{M}\left(\tilde{\chi}_{1}^{ \pm}\right)>190.4 \mathrm{GeV} / c^{2}$. Observed limits: $\mathrm{M}\left(\tilde{\chi}_{1}^{0}\right)>103.3 \mathrm{GeV} / c^{2}, \mathrm{M}\left(\tilde{\chi}_{1}^{ \pm}\right)>196.5 \mathrm{GeV} / c^{2} . \ldots \ldots \ldots 151$

7.11 Observed 95\% CL limits on the $\tilde{\chi}_{1}^{0}$ and $\tilde{\chi}_{1}^{ \pm}$masses for $M_{0}=$ $250 \mathrm{GeV} / c^{2}, \tan \beta=5, A_{0}=0 . \ldots \ldots \ldots \ldots \ldots \ldots$

A.1 Preliminary $\Delta \phi$ distribution including all trilepton events that fail

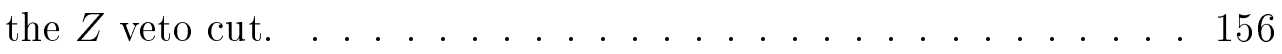

A.2 $Z / \gamma^{*}+\gamma \rightarrow \ell^{+} \ell^{-}+\gamma$ production at the Tevatron. The $\gamma$ can be produced through a) initial state radiation or b) final state radiation.160

A.3 Trilepton invariant mass for simulated $Z / \gamma^{*}+\gamma$ events. . . . . . 160

B.1 Method of selecting conversion candidates and determining correct and incorrect side. . . . . . . . . . . . 166

B.2 $\Delta Z$ between the seed electron and the CES partner cluster for the correct (circles) and incorrect (red line) side. The top plot shows the data and the bottom shows the MC. . . . . . . . 167 
B.3 Energy sharing between electron and positron in a photon conversions. A value of 0.5 corresponds to the electron and positron having equal energies. . . . . . . . . . . . . . 168

B.4 Background subtracted $\Delta \cot \theta$ and $D_{x y}$ for Data and MC. Each plot is filled once for each conversion candidate that has a nearby track. For both variables the width in $\mathrm{MC}$ is narrower than the width in

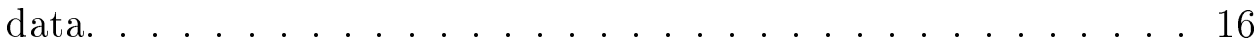

B.5 Background subtracted Data and MC distributions of photon conversion candidates: seed electron transverse energy (top left), CES partner cluster energy (top right), seed electron $E / p$ (bottom left), and seed electron fractional isolation (bottom right). The MC is normalized to the data by the number of events. The plots are filled once per conversion candidate. . . . . . . . . . . . . 170

B.6 Seed electron $\eta$ for data and MC. Plot is filled once for each candidate conversion event. . . . . . . . . . . . 171

B.7 Background subtracted radius of conversion for Data and MC. Plot is filled once for each seed electron that is tagged as a conversion. $\mathrm{MC}$ is normalized to data based on the number of events that convert at the COT endplate. . . . . . . . . . . . 172

B.8 A "brem" event where an electron radiates a photon. The photon will be on the correct side. . . . . . . . . . . . . 173

B.9 Two "trident" events where an electron radiates a photon that

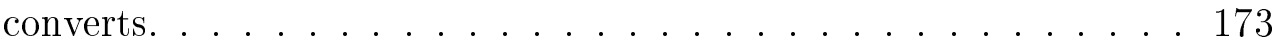


B.10 $\Delta Z$ between the seed electron and the CES partner cluster for the correct (circles) and incorrect (red line) side. The top plot shows MC Brem. events and the bottom shows MC trident. . . . . . . 174

B.11 $\Delta Z$ for events where the partner cluster has both an unbiased CES cluster and a track based CES cluster. The black line displays the correct side and the red line displays the incorrect side. . . . . . 177

B.12 Conversion removal efficiency for data and MC as a function of the

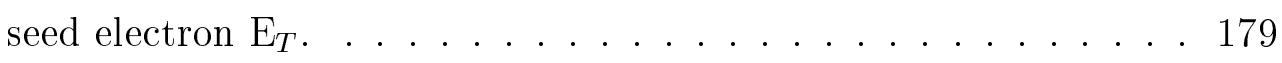

B.13 CES clusters for photon conversions belonging to the very low $\mathrm{p}_{T}$ sample: the blue line represents the seed electron, the red lines represent different scenario for the CES partner cluster. . . . . . . 180

B.14 Conversion electron $\mathrm{p}_{T}$ for the very low $\mathrm{p}_{T}$ sample. . . . . . . 181

B.15 Efficiency for the very low $\mathrm{p}_{T}$ sample as a function of the partner track minimum $\mathrm{p}_{T} \ldots \ldots \ldots \ldots$. . . . . . . . . . 182

B.16 Conversion removal scale factor as a function of the seed electron $\mathrm{E}_{T}$. Only the first $4 \mathrm{E}_{T}$ bins are used in the fit. The last bin is not used since the mean $\mathrm{E}_{T}=25 \mathrm{GeV}$ does not equal the bin center and would bias the fit. . . . . . . . . . . . 184

B.17 Conversion removal scale factor as a function of the seed electron $\mathrm{E}_{T}$. Only the first $4 \mathrm{E}_{T}$ bins are used in the fit. The last bin is not used since the mean $\mathrm{E}_{T}=25 \mathrm{GeV}$ does not equal the bin center and would bias the fit. . . . . . . . . . . 187 


\section{List of TABles}

2.1 Elementary particles of the standard model. . . . . . . . . . 8

2.2 There are 4 forces in nature, all of them except for gravity is contained in the SM. The signal strength depends on energy scale and is only listed to give an approximate idea of how they compare to each other. The graviton has not been discovered yet. . . . . . .

2.3 SM particles (left) and their corresponding superpartners in the $\operatorname{MSSM}($ right). . . . . . . . . . . . . . .

2.4 Production cross sections of superpartner pairs in proton-antiproton collisions at $\sqrt{s}=1.96 \mathrm{TeV}$ for $M_{0}=250, M_{1 / 2}=260, \tan \beta=5$, $A_{0}=0$, and $\mu>0$. Processes with smaller cross sections are not listed. ...................... 33

2.5 Limits on $\lambda_{121}$ from previous $\not R_{p}$ SUSY searches. . . . . . . . . 37

2.6 Limits on $\lambda_{122}$ from previous $\not R_{p}$ SUSY searches. . . . . . . . . 37

4.1 Lepton identification requirements for central electrons. . . . . . . 74

4.2 Lepton identification requirements for plug electrons. . . . . . . 75

4.3 Lepton identification requirements for stubbed muons. . . . . . . 77

4.4 Lepton identification requirements for stubless muons. . . . . . . 78

4.5 Parameterizations and fit values for the probabilities for jet candidates to be misidentified as leptons, for each lepton type. $\mathrm{E}_{T}$ and $p_{T}$ are measured in units of $\mathrm{GeV}$ and $\mathrm{GeV} / c$, respectively. . . . 
5.1 The mSUGRA input parameters (left) and resulting output quantities (right) for each MC sample generated for this analysis. $A_{0}=$ 0 for all points. Masses are calculated with ISAJET v7.51. The last column is the $\mathrm{NLO} \sigma\left(q \bar{q} \rightarrow \tilde{\chi}_{2}^{0} \tilde{\chi}_{1}^{ \pm}\right)+\sigma\left(q \bar{q}^{\prime} \rightarrow \tilde{\chi}_{1}^{ \pm} \tilde{\chi}_{1}^{\mp}\right)$ as determined

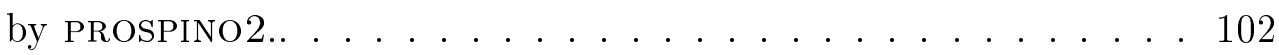

5.2 Trigger paths used in this analysis and their efficiencies. . . . . . 105

5.3 Background MC samples used in this analysis. The first column is the background process generated from the proton antiproton collision. The second column is the MC event generator used to simulate the events. The third column is the cross section times branching ratio of the particular process, and the last column is the integrated luminosity of the sample. . . . . . . . . 106

5.4 Lepton identification scale factors $\left(\epsilon_{\text {Data }}^{I D} / \epsilon_{M C}^{I D}\right)$ for each lepton type and $E_{T}$ or $p_{T}$ range. Scale factors are used to correct the MC event predictions due to differences with the data sample. . . . . . . 107

6.1 List of the dilepton control samples used in this analysis. . . . . . 115

6.2 List of the $\geq 3$-lepton control samples used in this analysis. . . . . 116

6.3 Numbers of predicted and observed trilepton events containing two opposite-sign same-flavor leptons that form an invariant mass between 76 and $106 \mathrm{GeV} / \mathrm{c}^{2}$. The control sample is broken down by lepton channel and background type. . . . . . . . . . . 117

6.4 Numbers of predicted and observed trilepton events containing two opposite-sign same-flavor leptons that have $160^{\circ}<\Delta \phi<200^{\circ}$. The control sample is broken down by lepton channel and background type. . . . . . . . . . . . . . . 117 
6.5 Systematic uncertainties associated with lepton identification for each lepton type and $\mathrm{p}_{T}$ range. . . . . . . . . . . . 122

6.6 Systematic uncertainties associated with the theoretical cross section for each process. The numbers include uncertainties from both $Q^{2}$ variation and variation between PDF sets. . . . . . . . 123

6.7 PYTHIA parameters used in determining the ISR and FSR systematic uncertainties for the $\not R_{p}$ SUSY signal samples. . . . . . . . 123

6.8 Contribution from each source of systematic uncertainty to the total uncertainty for both $\not R_{p}$ SUSY and background in each signal sample. . . . . . . . . . . . . . . . 131

7.1 Comparison of expected numbers of background events and $\not R_{p}$ SUSY prediction within the trilepton signal sample. The $\not R_{p}$ SUSY samples are generated with the following parameters: $M_{0}=250$, $M_{1 / 2}=260, \tan \beta=5, A_{0}=0$, and $\mu>0$....... 136

7.2 Comparison of expected backgrounds and $R_{p}$ SUSY prediction within the four or more lepton signal sample. The $R_{p}$ SUSY samples are generated with the following parameters: $M_{0}=250$, $M_{1 / 2}=260, \tan \beta=5, A_{0}=0$, and $\mu>0 \ldots \ldots 137$

7.3 Kinematic properties of the first three events in the trilepton signal samples. (*) The CMIO does not pass through the calorimeter and may be a misidentified electron or jet. . . . . . . . . . . . . 138

7.4 Kinematic properties of the last three events in the trilepton signal

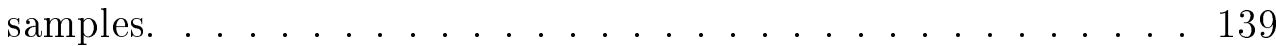


7.5 Expected and observed cross section upper limits determined at the $95 \%$ confidence level combining the 3 lepton and $\geq 4$ lepton signal samples for $\lambda_{121} . \quad \ldots \ldots \ldots \ldots \ldots$. . . . . . . . 145

7.6 Expected and observed cross section upper limits determined at the $95 \%$ confidence level combining the 3 lepton and $\geq 4$ lepton signal samples for $\lambda_{122} . \ldots \ldots \ldots \ldots$. . . . . . . . 146

7.7 Benefits of splitting the signal sample into two, one with exactly three leptons and one with four or more leptons. Expected and observed cross section limits are determined at 95\% CL for $M_{0}=$ $250 \mathrm{GeV} / c^{2}, M_{1 / 2}=260 \mathrm{GeV} / c^{2}, \mu>0$, and $A_{0}=0 \ldots \ldots 147$

7.8 Expected and observed 95\% CL limits on the $\tilde{\chi}_{1}^{0}$ and $\tilde{\chi}_{1}^{ \pm}$masses for $M_{0}=250 \mathrm{GeV} / c^{2}, \tan \beta=5, A_{0}=0 \ldots \ldots \ldots \ldots$

A.1 Preliminary comparison between the background prediction and data for all trilepton events that fail the $Z$ veto cut. $\ell$ denotes electrons or muons. . . . . . . . . . . . . . . . . . 157

A.2 Preliminary comparison between the background prediction and data within the trilepton signal samples. . . . . . . . . . . 158

A.3 Preliminary comparison between the background prediction and data for trilepton events which fail the $\Delta \phi$ requirement, yet pass the $Z$ veto cut. . . . . . . . . . . . . . . . . 159

A.4 Remaining events from Table A.2 after rejecting events with $76<$ $\mathrm{M}_{\ell^{+} \ell^{-} e}<106 \mathrm{GeV} / c^{2} \ldots \ldots \ldots \ldots \ldots 1$ 
B.1 Number of brem and trident events in DY MC that have a seed electron + CES partner cluster. We split the events into correct (conversion candidate) and incorrect side (background candidate) depending on the relative position of the partner cluster and whether the seed electron is tagged as a conversion. A conversion candidate (or background) is considered tagged if it passes the conversion removal cuts. . . . . . . . . . . 175

B.2 Conversion removal efficiency in Data and MC. . . . . . . . . 178

B.3 Conversion removal efficiency in Data and $\mathrm{MC}$ when a min $\mathrm{p}_{T}$ cut is applied. . . . . . . . . . . . . . . . . 181

B.4 Efficiency of each conversion requirement. Cuts are applied se-

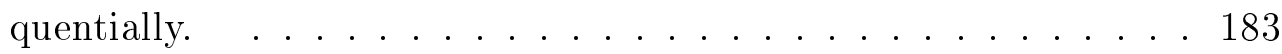




\section{ACKNOWLEDGMENTS}

The completion of the work presented in this document would have never been realized if it were not for the help and inspiration of many others along the way. First of all, I would like to thank my advisor Jay Hauser for having the common sense to provide relevant advice and feedback when it was needed and to leave me alone when it wasn't.

I owe a great deal of gratitude to Mike Lindgren for taking me under his wing early on and showing me that working doesn't have to be serious all the time. I would also like to thank Beate Heinemann for being a wealth of information and encouragement.

It was my pleasure to work closely with the SUSY-Multilepton group: Anadi Canepa, Sourabh Dube, Else Lytken, John Strologas, Giulia Manca, Melisa Rossi, Martin Griffiths, Oscar González López, Vladimir Rekovic, and John Zhou. They made my time at Fermilab much more enjoyable.

I would like to acknowledge everyone that I worked with from UCLA, especially Brian Mohr for hanging out with me either at the office or in Chicago for a night of blues. Also Charles Plager and Peter Dong for our fun and zany conversations.

Lastly, I would like to thank my parents Susan and Lucien Attal for supporting me unconditionally. My appreciation for them is much more than I could ever express. 


\section{VITA}

Born, Los Angeles, California.

1999

B.S., Physics

University of California, San Diego

1999

Alyne and Leon Camp Graduate Fellowship

University of California, Los Angeles

2001

M.S., Physics

University of California, Los Angeles

\section{Publications and Presentations}

A. Attal, Search for Anomalous Production of Multi-lepton Events at CDF. Presented at the Joint Meeting of Pacific Region Particle Physics Communities, Honolulu, Hawaii, October 2006.

A. Attal, Search for R-Parity Violating Supersymmetry. Presented at the UCLA High Energy Physics Seminar, Los Angeles, California, May 2006.

A. Attal, Search for R-Parity Violating SUSY in the Four Lepton Final State at CDF. Presented at the Pheno Symposium, Madison, Wisconsin, April 2006.

A. Attal, Search for R-Parity Violating SuperSymmetry in the Four-Lepton Final 
State at the Tevatron. Presented at the meeting of the American Physical Society, Tampa, Florida, April 2005.

A. Attal, "Plug Shower Maximum Detector for CDF Run II" in Proceedings of the Tenth International Conference on Calormietry in Particle Physics:557-562, March 2002.

A. Attal, Plug Shower Maximum Detector for CDF Run II. Presented at the Tenth International Conference on Calorimetry in Particle Physics, Pasadena, California, March 2002.

xxvi 


\title{
Abstract of the Dissertation \\ Search for R-Parity Violating Supersymmetry in the Multilepton Final State
}

by

\author{
Alon Jacques Attal \\ Doctor of Philosophy in Physics \\ University of California, Los Angeles, 2006 \\ Professor Jay Hauser, Chair
}

This thesis presents a search for physics beyond the standard model of elementary particles in events containing three or more charged leptons in the final state. The search is based on an R-parity violating supersymmetric model that assumes supersymmetric particles are pair produced at hadron colliders and the R-parity violating coupling is small enough so that these particles "cascade" decay into the lightest supersymmetric particle. The lightest supersymmetric particle may only decay into two charged leptons (electrons or muons) plus a neutrino through a lepton number violating interaction. Proton-antiproton collision events produced with $\sqrt{s}=1.96 \mathrm{TeV}$ are collected between March 2002 and August 2004 with an integrated luminosity of $346 \mathrm{pb}^{-1}$. R-parity violating supersymmetry is sought for in two data samples, one with exactly three leptons and one with four or more leptons. The trilepton sample has a modest background primarily from Drell-Yan events where an additional lepton is a result of photon conversions or jet misidentification while the four or more lepton sample has an extremely low background. In the three lepton samples 6 events are observed while in the four or more lepton sample zero events are observed. These results are consistent with 
the standard model expectation and are interpreted as mass limits on the lightest neutralino $\left(\tilde{\chi}_{1}^{0}\right)$ and lightest chargino $\left(\tilde{\chi}_{1}^{ \pm}\right)$particles. The $\tilde{\chi}_{1}^{0}$ mass is constrained to be heavier than 97.7 to $110.4 \mathrm{GeV} / c^{2}$, while the $\tilde{\chi}_{1}^{ \pm}$mass is constrained to be heavier than 185.3 to $202.7 \mathrm{GeV} / c^{2}$, depending on the supersymmetry scenario. 


\section{CHAPTER 1}

\section{Introduction}

In my mind nature is the grandest form of art in existence. From galaxies, to trees, to atoms and particles, nature contains beauty at all scales. The more we study and understand nature, the more beautiful it becomes, adding to our appreciation. Take an oak tree for example. At first glance it may look daunting and majestic with its broad trunk, many branches, and abundance of leaves. Upon closer examination, we see deep grooves in the trunk, the rich complexity in the leaves of each vein running along the surface or its distinctive shape, and depending on the time of year there may be acorns on the branches as well. When I think that each individual acorn has the potential to become another oak tree, it amazes me. How can all the oak's beauty be contained in such a small package? It seems like the more closely we look, the more there is to discover. To quote Calvin \& Hobbes [1], "There's treasure everywhere!" For me it was quite natural to study nature at the smallest scale there is, the particle level. As far as we know, everything in the universe is composed of particles. Therefore, by studying the properties and interactions of particles, we gain an appreciation for everything.

At the heart of particle physics is the Standard Model (SM). Contained in the SM are three fundamental forces: the strong nuclear force, the weak nuclear force, and electromagnetism and three types of particles: leptons, quarks, and bosons. The SM will be discussed in more detail in Section 2.3. While the SM is enormously successful in its power to predict experimental quantities, it does not 
explain everything and an essential piece of it is still missing in action. The piece which is being actively sought after is the Higgs boson particle, the part of the SM that explains how particles acquire mass. While there is no guarantee that the Higgs exists, there is an elegance about how it ties up the loose ends of the SM which draws physicists to believe it is out there and worth spending years to try and find. But even with the Higgs, the SM lacks completeness. There are clues that there may be physics beyond the SM. It is like a performance car which is well tuned, smooth running, yet there seems to be a faint "hum" emanating from the rear. While the source of the humming sound could be due to grooves in the road, there is also the possibility that it is a sign of a subtle defect in the car's frame. Clearly, we should not ignore the clue, we should strive to understand the nature of it. Even if we do not find the source of the hum, we may find something else undiscovered, and we will definitely improve our appreciation for the car. After all, the more you look...

Gone are the times where one or two scientists can do a high energy physics experiment which has not been done before in a single room office laboratory overnight. We have entered a new era in particle physics, one of worldwide collaborations, kilometer long accelerators, and mammoth detectors. So when our car, that we named the Standard Model, has an element of mystery that is driving us crazy, we take it to one of these particle acceleration facilities. The first thing a good mechanic does, is assess the clues to think of possible reasons for the hum. Once a list of theories have been compiled, the mechanic will test each of them until one comes back positive. In high energy physics, physicists work in the same way. Theorists establish possible scenarios which would explain the perplexities of the SM and experimentalists test these theories using our huge laboratories. In the process we will expand our appreciation for particles and everything built from particles. 
This thesis tests a theory which describes physics not contained by the Standard Model. The theory is called SuperSymmetry (SUSY), described in Section 2.11. While there are several supersymmetric theories, SUSY generally predicts a new particle for every particle contained in the SM. We can test the hypothesis that there are additional particles by searching for experimental deviations from the SM. One such deviation is the number of leptons which can be detected by the Collider Detector at Fermilab ( $\mathrm{CDF}$ ) experiment located at the Fermi National Accelerator Laboratory (FNAL) in Batavia, IL. For example, the SM predicts that a certain number of leptons will be produced in particle collisions while SUSY predicts a larger number of leptons will be produced. If the lepton production is measured to be consistent with the SUSY prediction, and not the $\mathrm{SM}$, then this is evidence of new particles. In this thesis I search for collision events where we detect three or four leptons, either electrons or muons, to verify or partially rule out whether SUSY or other new physics models predicting multilepton production explain some of the questions physicists have with the SM.

We all know, or at least can imagine, how frustrating it can be to take our cars to the shop or go to a doctor with a particular symptom in mind only to hear "there's nothing wrong." The reason for this frustration is that the statement is not very accurate and is often simply wrong. It does not explain anything about the symptom or why it is there. A more accurate statement would explain what possible causes have been ruled out and to what precision. In physics, we place a great deal of effort in understanding and reporting the accuracy of our measurements. To obtain the maximum accuracy, we need to understand as much of the experiment as possible and double check for mistakes. Simply searching for three and four leptons in the data without preparation would be both a waste of time and provide misleading results. This thesis describes each step taken 
in a search for R-Parity Violating SUSY. The outline is as follows: Chapter 2 describes in more detail the SM, SUSY, and particle physics in general; Chapter 3 describes how collision events are produced at FNAL and how they are processed by the CDF detector; Chapter 4 explains how information taken from the CDF detector is used to identify particles, specifically leptons; Chapter 5 goes into detail about which data samples are used, how SUSY events are simulated, and what SM processes mimic SUSY events; Chapter 6 describes how the search for SUSY events within the data samples is done; Chapter 7 explains the results of the search; and in Chapter 8 are the conclusions. 


\section{CHAPTER 2}

\section{Theory \& Motivation}

This chapter provides a brief explanation of particle physics, why physicists believe that there is more to be discovered, why it is interesting to search for extensions beyond the Standard Model, and a short discussion of supersymmetry. High energy particle physics is based on a number of concepts such as general

physics, mathematics, quantum mechanics, and special relativity. To cover all of these topics is not practical considering the desired length of this dissertation. For those interested in learning more including a historical perspective, there are excellent introductory books on quantum mechanics [2] and elementary particle physics $[3,4,5]$.

\subsection{What is a particle?}

As far as we know, particles are the most fundamental, indivisible constituents of the universe. Because of their minuscule size, particles have to be described by quantum mechanics (physics at small distances). This results in particle behavior that can be counterintuitive, such as particle/wave duality, where a particle exhibits properties of both classical particles like billiard balls and waves like ripples in water. Individual particles are identified by their mass, charge, spin, 
and color ${ }^{1}$, among others. While many different types of particles exist, particles of the same type are indistinguishable from each other since they have exactly the same properties.

\subsection{Spin \& the Pauli Exclusion Principle}

Of particular interest in this study is the spin quantum number. Spin refers to the angular momentum of the particle along the axis of rotation in units of $\hbar$. A distinction is made between particles with half integer spin, known as fermions and particles with whole integer spin known as bosons. For a spin 1 particle, rotating it by $360^{\circ}$ returns it to its initial state, while for a spin $\frac{1}{2}$ particle, it must be rotated by $720^{\circ}$ to return to its initial state. According to the Pauli exclusion principle, identical fermions can not be in the same place at the same time. As a consequence, fermions are essentially building blocks and can be stacked "on top" of each other. Alternatively, bosons may freely travel right through each other, making them ideal messengers.

Related to spin is the concept of helicity. For spin $\frac{1}{2}$ particles, the $\mathrm{z}$ component of the spin can either be $+\frac{1}{2}$ or $-\frac{1}{2}$. This is why in chemistry two electrons occupy each orbital, one for spin up and one for spin down. Likewise, a spin 1 particle can have three values for the $\mathrm{z}$ component of spin: 0 and \pm 1 . If the direction of a particle $(\hat{v})$ is parallel to the direction of spin $(\hat{z})$, then the particle has positive helicity (right-handed), otherwise if they are anti-parallel, it has negative helicity (left-handed), see Fig. 2.1.

\footnotetext{
${ }^{1}$ color refers to the charge of the strong nuclear interaction and not classical colors of light like red, blue, and yellow
} 


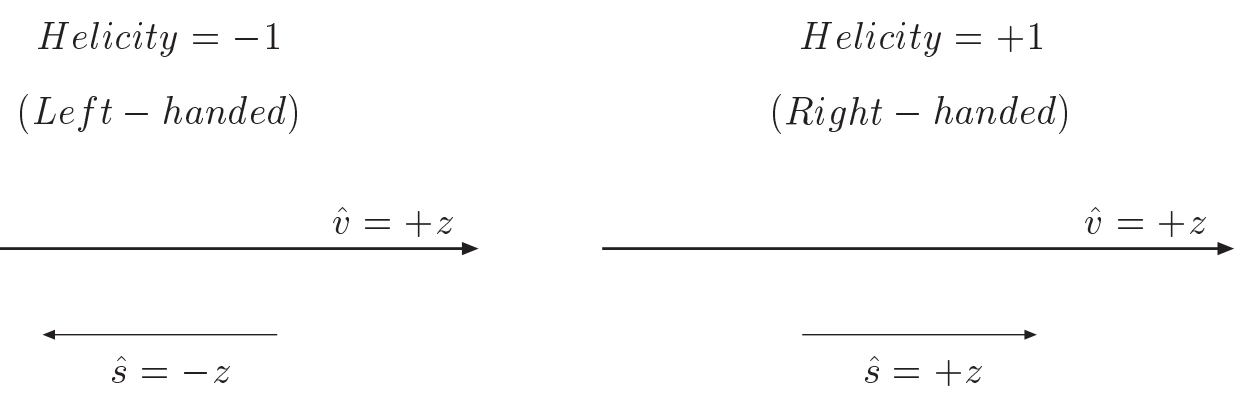

Figure 2.1: Definition of helicity, where $\hat{v}$ is the particle direction and $\hat{s}$ is the component of spin along $\hat{v}$. Figure adapted from [5].

\subsection{Standard Model of Particle Physics}

The standard model of particle physics, referred to in this publication as the SM, contains all of the known elementary particles and their interactions (excluding gravity). A particle is considered elementary if it is not composed of any other particles. The SM includes three families of quarks and leptons, both of which are fermions. In the first family, the up quark $(u)$ has an electric charge of $+\frac{2}{3} e$ while the down quark $(d)$ has a charge of $-\frac{1}{3} e$, where in this context $e$ refers to the charge of an electron equal to $1.6 \times 10^{-19} \mathrm{C}$. All quarks carry one unit of color, either red, blue, or green. The electron $(e)$ has an electric charge of $-1 e$ and the electron neutrino $\left(\nu_{e}\right)$ is electrically neutral and is much lighter than the electron. The other families are identical to the first one except for mass, with the first family being the lightest and the third the heaviest. In addition there are also four bosons which mediate interactions between fermions: photon $(\gamma)$, gluon $(g)$, $W^{ \pm}$, and $Z$. The particles of the SM are listed in Table 2.1.

The SM also contains antiparticles for each particle. Antiparticles are identical to their particle relatives except they have opposite charges. For example, the antiparticle of an electron is a positron with the same mass but positively charged 


\begin{tabular}{lccc|c}
\hline & \multicolumn{3}{c|}{ Family } & Messengers \\
& I & II & III & (bosons) \\
\hline Quarks & $u$ & $c$ & $t$ & $\gamma$ \\
(fermions) & $d$ & $s$ & $b$ & $\mathrm{~g}$ \\
\cline { 1 - 2 } Leptons & $e$ & $\mu$ & $\tau$ & $W$ \\
(fermions) & $\nu_{e}$ & $\nu_{\mu}$ & $\nu_{\tau}$ & $Z$ \\
\hline
\end{tabular}

Table 2.1: Elementary particles of the standard model.

and the anti-up quark has $-\frac{2}{3} e$ charge and has one unit of anti-color, either antired, anti-blue, or anti-green. In some instances, like the photon, the particle has exactly the same properties as the antiparticle. To distinguish between particles and antiparticles, a bar is often placed over the symbol. For example, $\bar{b}$ represents an anti- $b$ quark.

There are four forces, or interactions, that are listed in Table 2.2, of which three are contained within the SM: electromagnetic, weak and strong. At the particle level, each of these forces can be described by an exchange of a boson. The electromagnetic force between charged particles is mediated by photons. Electromagnetism is responsible for phenomena at the particle level such as binding electrons to nuclei to form atoms and for phenomena at the macroscopic level such as lightning. The strong force between colored particles is mediated by gluons and is responsible for binding quarks together to form protons and neutrons and for binding protons and neutrons together to form atomic nuclei. The weak force that affects all leptons and quarks is mediated by the $W$ and $Z$ bosons. The radioactive process called beta decay in which an electron is emitted, is a signature of the weak force. 


\begin{tabular}{lcc}
\hline Force & Mediator & Approximate Strength \\
\hline Strong & gluon $(\mathrm{g})$ & $10^{1}$ \\
Electromagnetic & photon $(\gamma)$ & $10^{-2}$ \\
Weak & $\mathrm{W}^{ \pm}, \mathrm{Z}^{0}$ & $10^{-5}$ \\
Gravity & graviton $(\mathrm{G})$ & $10^{-40}$ \\
\hline
\end{tabular}

Table 2.2: There are 4 forces in nature, all of them except for gravity is contained in the SM. The signal strength depends on energy scale and is only listed to give an approximate idea of how they compare to each other. The graviton has not been discovered yet.

\subsection{Asymptotic Freedom \& Color Confinement}

The theory of the strong interaction is known as quantum chromodynamics (QCD). Without going into much detail about QCD, there are some other important properties of the strong interaction that are worth mentioning, namely asymptotic freedom and quark confinement, since they will be important later in this thesis. The strength of the strong interaction is larger at long distances and smaller at short distances, which is contrary to the electromagnetic interaction where electric charges are more attracted or repelled to each other depending on their distance from each other and the signs of their charges. Therefore, quarks that are close to each other act like free particles, but if one attempts to separate them it becomes increasingly difficult, as if stretching a rubber band. This phenomena is called asymptotic freedom. For this reason, quarks are never found individually. Instead, they are grouped into color neutral particles: quarkantiquark pairs (mesons) or in quark triplets (baryons). Collectively, any particle containing a quark is known as a hadron. Color neutral particles are produced in 
mesons by having one quark with a certain color and another quark with the anticolor or in baryons by having each quark with a different color. This property is known as color confinement.

\subsection{Particle Interactions}

In particle physics there are three basic types of interactions involving the fundamental forces of the SM: decays, scattering, and bound states. The particles before and after the interaction are referred to as the initial and final state respectively. In a vacuum, particles have energy $(E)$ given by $E^{2}=p^{2} c^{2}+m^{2} c^{4}$, where $p$ and $m$ are the particle's momentum ${ }^{2}$ and mass, respectively, and $c$ is the speed of light. When they interact, individual particles can be created or destroyed as long as certain quantities are conserved such as energy, momentum, and charge. Decays occur when a single heavy particle decays into two or more lighter particles. A particle's decay rate $(\Gamma)$ is defined as the probability per unit time that the particle will decay and its lifetime is $1 / \Gamma$. If the particle does not decay it is referred to as stable.

Scattering involves interactions between two or more particles where the final state particles are not bound together. A useful quantity in scattering is the cross section $(\sigma)$ which has units of 1 /Area and is a measure of how likely particles interact given their proximity to each other, energy, and momentum. The cross section also depends on the particles in the final state. Given two initial particles, there may be many possible outcomes each with its own cross section. The larger the cross section for each final state, the higher the probability that the particular interaction will occur.

\footnotetext{
${ }^{2}$ Momentum $(\vec{p})$ is a vector, meaning it contains both magnitude $p$ and direction $\hat{p}$ information.
} 
Bound states involve two or more particles, where the energy required to separate them is larger than the energy needed to hold them together. Protons are bound states of two $u$ quarks and one $d$ quark held together by the strong interaction. Another common bound state is the hydrogen atom where an electron and a proton are bound together by the electromagnetic interaction.

\subsection{Proton Antiproton Collisions}

Since this analysis is conducted at the Fermilab Tevatron, special attention is focused on interactions of high energy protons and antiprotons. To answer the question of what occurs during this process, the first thing to consider is the structure of the protons and antiprotons. Due to the principles of asymptotic freedom and quark confinement, described in Sec. 2.4, explaining the structure of these two particles is more complicated than it seems. For one thing, protons and antiprotons are more than the product of the basic "valence" quarks which consist of two $u$ quarks and one $d$ quark for the proton and two $\bar{u}$ quarks and one $\bar{d}$ quark for the antiproton. From QCD, it is known that there are a "sea" of gluons within the proton or antiproton that travel between each of the valence quarks or between other gluons. In addition there is a "sea" of quarks which can be produced within the gluon interactions. The quarks and gluons within this sea are not permanent like the valence quarks but are constantly being produced and annihilated. Even heavier quarks such as the $s$ may be found inside the proton but with low probability. Each of these constituents, also known as partons, contain only a fraction of the total proton or antiproton momentum and therefore it is convenient to introduce the dimensionless variable $x$, defined as $x \equiv p_{z} / p$, which measures the fraction of the beam's momentum $(p)$ which is contained in the component of the constituent's momentum along the direction of the beam 
$\left(p_{z}\right)$ [6]. For example, if $x$ is $1 / 3$ for one of the valence quarks, it means that the quark has a third of the momentum of the proton in the direction of the beam. To calculate cross sections involving hadrons, particle physicists use parton distribution functions (PDFs), which describe the probability to find a certain constituent within a hadron as a function of $x$.

For hadron interactions, the inclusive cross section is higher at small values of $x$ than it is for higher values [6]. Therefore, most collisions between high energy hadrons are "soft," meaning that only a small amount of momentum is transferred from the partons within the proton and antiproton into new particles. It is useful to distinguish between the beam direction $(z)$ and the transverse direction $(T)$ perpendicular to the beam direction. For the most part, minimum bias events are produced in proton-antiproton collisions characterized by a multiplicity of low- $p_{T}(<2 \mathrm{GeV} / c)$ charged and neutral particles. Every once in a while a collision will occur with high $x$ partons leading to a "hard" interaction, characterized by high- $p_{T}$ particles in the final state. During the hard scattering a quark or gluon may be emitted from the proton or antiproton. Due to the nature of the strong force, an isolated quark or gluon essentially has infinite potential energy. This potential energy is used to produce quark-antiquark pairs out of the vacuum which can combine with the free quark or gluon to produce color neutral hadrons, in a process called hadronization. So instead of seeing a free quark or gluon in a particle detector, what is observed is a group of hadrons, traveling in approximately the same direction. Collectively, these particles form what is called a jet. The composition of the jet depends on the momentum and type of the initial quark or gluon. Of all the hadrons produced within jets, the most common are low mass mesons called pions, which are spin 0 particles formed from $u$ and $d$ quarks and can have electric charge $\left(\pi^{ \pm}\right)$or be neutral $\left(\pi^{0}\right)$. 
As a result of a hard interaction, the proton and antiproton disintegrate and their remnants may interact with each other. This is known as the underlying event. The $p_{T}$ of the particles produced in the underlying event depends on the values of $x$ of the remaining partons.

Since quarks have both electric and color charges, they may interact via the electromagnetic, weak, or strong interactions, although the latter dominates, while gluons can only interact through the strong force. While less common, particles can also be created through the electromagnetic or weak interactions. For example, two oppositely charged quarks annihilate to form a $\gamma^{*}$ or $Z$ boson, or two quarks where the sum of the electric charges is \pm 1 annihilate to form a $W$ boson. The $\left(^{*}\right)$ of $\gamma^{*}$ indicates that the photon is a virtual particle. Virtual particles, which are never observed directly, can be created even when there is insufficient energy to produce them normally. Virtual particles are also called offmass shell particles since their masses may differ from experimentally measured values [7].

\subsection{Symmetry}

Symmetry is an important aspect of both art and nature. An object is said to have symmetry if it can be transformed while leaving it in the same state that it started in. For example, a snowflake has rotational symmetry, since turning it by $60^{\circ}$ does not change its appearance ${ }^{3}$, see Fig. 2.2. Another example of a symmetry is mirror symmetry, or parity. An object has parity, if its mirror image behaves exactly the same as the object itself. There are also mathematical symmetries, for instance a trivial example is to multiply any number by 1 .

\footnotetext{
${ }^{3} \mathrm{~A}$ typical snowflake only has approximate rotational symmetry due to small differences between the branches. Also there are many types of snowflakes, like needle snowflakes, that do not have 6-fold symmetry.
} 
In physics, symmetries play an important role in understanding particles and their interactions due to a connection between symmetry and conserved quantities discovered by Emmy Noether in 1917 [8]. Noether's theorem states that for every continuous symmetry in nature there is a corresponding conserved quantity. For example, rotational symmetry leads to a conservation of angular momentum.

In quantum mechanics, the equations of physics include $\bar{\psi} \psi$, where $\psi$ is a complex wave function which depends on space and time, and $\bar{\psi}$ is the complex conjugate of $\psi$. There may be additional symmetries if this wave function can be modified in such a way that the laws of physics remain invariant. For example, if the wave function is transformed by a global gauge, corresponding to a complex rotation:

$$
\psi \rightarrow e^{i \alpha} \psi
$$

the product $\bar{\psi} \psi$ is unchanged. The class of phase transformations $e^{i \alpha}$, forms the unitary group, $\mathrm{U}(1)$ [9]. If instead the wave function is transformed by a local gauge:

$$
\psi \rightarrow e^{i \alpha(x)} \psi
$$

the situation becomes more complicated since both $\psi$ and $e^{i \alpha(x)}$ depend on position. In order to preserve the laws of physics under local gauge invariance, a massless field must be introduced. This is important since the invariance under a local U(1) transformation corresponds to the electromagnetic interaction, where the massless field is in fact the photon, and the associated conserved current corresponds to the electric charge.

In a similar fashion we can relate the other forces in the SM to phase transformation symmetries: the weak interactions by a $\mathrm{SU}(2)$ symmetry and the strong interactions by a $\mathrm{SU}(3)$ symmetry. The $\mathrm{SU}(\mathrm{n})$ group contains all $\mathrm{n} \times \mathrm{n}$ unitary matrices with determinant 1 and possessing $n^{2}-1$ degrees of freedom. For each 


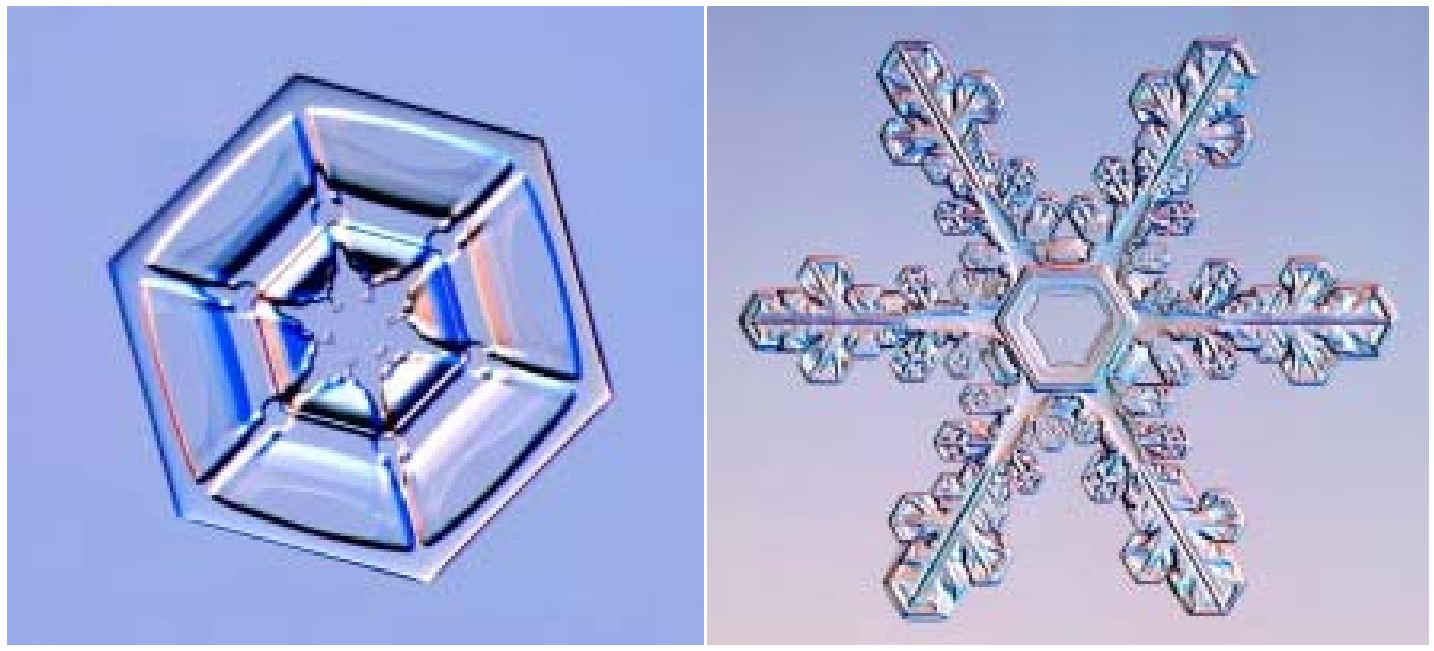

Figure 2.2: The snowflake has rotational symmetry, such that rotating by $60^{\circ}$ leaves it invariant. (Reprinted by permission of snowcrystals.com).

degree of freedom there is a corresponding messenger particle and therefore there are 3 weak gauge bosons $\left(2^{2}-1\right)$ and 8 gluons $\left(3^{2}-1\right)$.

\section{Parity Violation}

Parity involves taking the mirror image of an object. In particle physics, this corresponds to turning a right-handed particle into a left-handed particle. While the electromagnetic and strong forces preserve parity invariance, it is violated by the weak force [10]. In weak interactions, left-handed and right-handed particles are treated differently and as a result only left-handed neutrinos and right-handed antineutrinos have been observed in experiments.

\subsection{Higgs Field}

One of the great successes of the SM is its compactness while still being able to describe almost everything that goes on at the particle level. Yet there still 
remains a piece which is yet to be verified by experiment. It has to do with particle masses, ranging from the mass of the neutrino $\left(<0.75 \mathrm{eV} / \mathrm{c}^{2}[11]\right)$ to the mass of the top quark $\left(\sim 175 \mathrm{GeV} / \mathrm{c}^{2}[12]\right)$. According to the SM, if there are only three types of interactions, excluding gravity, then all the particles should be massless, otherwise local gauge invariance would be broken. Therefore there must be other particles/interactions, that explain why particles have mass and why some particles have different masses than others, while preserving local gauge invariance.

One possible solution is that of the Higgs field [13, 14]. To help explain the Higgs field, it is useful to compare it to an electric field, which is well understood. Electric fields are emitted by charged particles and decrease in strength the farther one moves away from the charge. If another charged particle moves within the field, it will be either be attracted or repelled to it by a force proportional to the strength of the charge. The Higgs field is different because it is not localized around "charged" particles, instead it has the same value everywhere in space. Similar in the way that the electric field only affects charged particles, the Higgs field only affects massive particles with a strength proportional to the mass of the particle. In fact it is the interaction of the Higgs field with certain particles that results in mass. To get a better feeling for how the Higgs field works, imagine walking through a pool of water. It is much more difficult to walk through water than it is through air since it is a denser substance. It is as if you have more mass walking through water than walking through air. Analogously, it is the Higgs field which makes it more difficult for massive particles to travel through it, essentially adding mass. For really light particles, the Higgs field will appear as air, but for very heavy particles, the Higgs field will seem to be a thick sticky fluid. 
As mentioned before, the electric field actually consists of bosonic particles called photons. And just like the electric field is associated with a photon there must be a particle associated with the Higgs field too, called the Higgs boson. Since the Higgs boson interacts with the Higgs field, it too will have mass. While the Higgs field provides an elegant way of explaining particle mass while preserving local gauge invariance, it does not predict what the particle masses should be. So far, all the known masses have been measured through experiments. The same will be true for the Higgs boson, which must at least be heavy enough to explain why it has not been discovered yet.

\subsection{Spontaneous Symmetry Breaking (Higgs Mechanism)}

In Section 2.7, the role of symmetries in particle physics was described. It turns out that spontaneous symmetry breaking can have important implications as well. Symmetry breaking describes a process that results in an object no longer possessing a symmetry it once had. For example, consider a plastic ruler that has left-right symmetry since one side is exactly the same as the other. But, if the ruler is pressed simultaneously on both ends, it may bend in one direction, destroying its left-right symmetry. This is known as spontaneous symmetry breaking.

In particle physics, the concept of spontaneous symmetry breaking is used by the Higgs mechanism to explain how particles can be massive while preserving local gauge invariance [5]. To understand the Higgs mechanism, consider a one

dimensional potential which depends on the spin 0 field $\phi$, shown in Fig. 2.3. The potential has the form,

$$
V(\phi)=-\frac{1}{2} \mu^{2} \phi^{2}+\frac{1}{4} \lambda^{2} \phi^{4},
$$




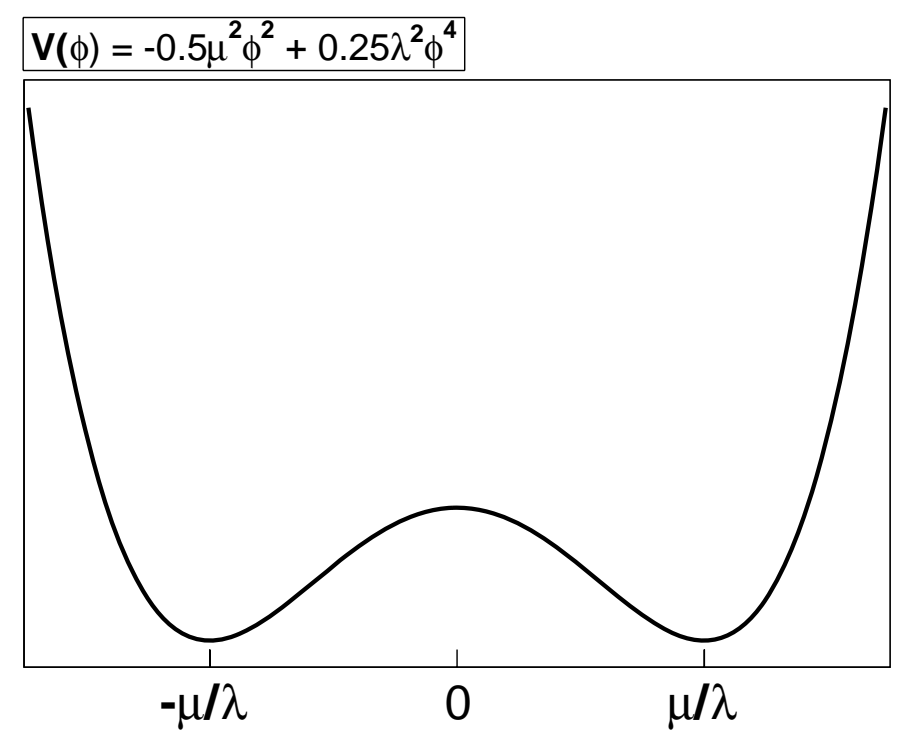

Figure 2.3: One dimensional scalar field $(\phi)$ potential, adapted from [5].

which has two minima at $\phi= \pm \mu / \lambda$ and a local maximum at $\phi=0$. Now imagine a ball that moves along the shape of the potential. If the ball sits exactly at the center at $\phi=0$, then there is a left-right symmetry since $\mathrm{V}(\phi)=\mathrm{V}(-\phi)$. But if the ball is pushed to one side and does not have enough energy to make it back to the top at $\phi=0$, it will fall to one of the two minima and the symmetry will be spontaneously broken, just like in the example of the ruler. In particle physics, the ball corresponding to the scalar field $\phi$, is an actual particle. The lowest energy the particle can have is $\mathrm{V}(\phi= \pm \mu / \lambda)=-\frac{1}{4} \mu^{4} / \lambda^{2}$, also known as its vacuum expectation value (VEV).

The next step is to generalize the potential in Equation 2.3 from one to two dimensions. The two dimensional potential is described by,

$$
V(\phi)=-\frac{1}{2} \mu^{2}\left(\phi_{1}^{2}+\phi_{2}^{2}\right)+\frac{1}{4} \lambda^{2}\left(\phi_{1}^{2}+\phi_{2}^{2}\right)^{2}
$$

This is analogous to replacing the plastic ruler with a round plastic straw, which has continuous rotational symmetry since turning it by any angle along its length 
leaves it invariant. If the the straw is pressed at both ends, than it can bend in not one of two directions, but one of many directions, spontaneously breaking the symmetry. Now that there are two dimensions, there are two new particles, one which will correspond to the Higgs particle and another which corresponds to a particle called the Goldstone boson. According to the Higgs mechanism, the Goldstone bosons are "eaten" in order to provide particle mass. While this may seem strange, this is what indeed happens in quantum field theory, where the end result includes terms equivalent to particle mass, only one of the two fields introduced in the Higgs potential remain, and the whole thing is locally gauge invariant.

To summarize, the Higgs mechanism describes how spontaneous symmetry breaking results in massive particles. This concept is vital to the SM, since the electromagnetic force and the weak force were shown by Glashow, Weinberg, and

Salam to be two parts of a single electroweak force [15], which is spontaneously broken at a certain energy known as the electroweak scale. Electroweak symmetry breaking allows the mediators of the weak force $\left(W^{ \pm}, Z^{0}\right)$ to be massive while all the other mediators are massless. Another important concept introduced in the Higgs mechanism is the VEV. All other SM bosons, except for the Higgs have a $\mathrm{VEV}=0$. This explains why the Higgs field exists everywhere even when there is no Higgs particle present. The same is not true of the electromagnetic, weak, and strong forces. For example, no electric field is observed unless there is a charged particle present.

\subsection{Motivation for Physics Beyond the Standard Model}

The Higgs boson may be the last piece of the SM, but perhaps there are other phenomena that can not be explained. In this section, I describe a few of the 
clues that suggest there may be physics beyond the SM [16]:

- Quantum Gravity: The biggest reason particle physicists believe that there is "new" physics is because the SM does not include gravity. It is suspected that a new type of particle interaction emerges around the Planck Scale $\left(\sim 10^{19} \mathrm{GeV}\right)$ that unites the theories of gravity and quantum mechanics. So far, the concept of string theory [17] seems like the most promising method of introducing a quantum theory of gravity. As the theory exists today, in order for string theory to describe our physical universe, supersymmetry is required, which is introduced in the next section.

- Hierarchy Problem: According to quantum field theory, the Higgs mass depends quadratically on the scale where new physics appears $(\Lambda)$. If $\Lambda$ is equal to the Planck Scale, then we would expect the Higgs mass to be too heavy to adequately explain the masses of known particles such as electrons and $\mathrm{Z}$ bosons, unless the theory is "fine tuned" so that by some improbable coincidence the particle mass spectrum comes out correctly. This is known as the hierarchy problem. For the mass of the Higgs to be consistent with the SM, a more natural value of $\Lambda$ would be much closer to the electroweak scale $(\sim 100 \mathrm{GeV})$.

- Dark Matter: On a cosmological scale, objects that travel around galaxies have angular velocities that depend on the amount of mass $(\mathrm{M})$ within the orbit and the distance from the center of the galaxy (r). Yet, in most galaxies the angular velocity does not coincide with what is expected after measuring $\mathrm{M}$ and $\mathrm{r}$. The most logical explanation is the presence of dark matter, which is not visible by telescope, and therefore not included in the calculation of M. More recent evidence for the existence of dark matter 
comes from anisotropy measurements of the cosmic microwave background (CMB) by the WMAP satellite [18]. According to a cosmological model known as the "Big-Bang", the universe was once hot and dense and has expanded and cooled to what we now observe [19]. The CMB is proposed to be the relic background radiation from the Big-Bang [20]. The measurements by WMAP indicate that $22 \%$ of the universe is composed of dark matter. None of the particles within the SM has the necessary characteristics to describe this phenomena.

- Particle Asymmetry: In the present universe there are more particles than antiparticles in contradiction with the Big-Bang model where the universe was once composed of equal quantities of matter and antimatter. For this asymmetry to manifest, there must be interactions which treat particles and antiparticles differently, known as $\mathrm{CP}$ violation. Although the weak interaction includes $\mathrm{CP}$ violation, it is not enough to account for the current particle asymmetry.

- Particle Families: The SM does not explain why there are three families of quarks and leptons. Just like the 6-fold symmetry of a snowflake indicates that there is an underlying property of the crystals that make up a snowflake, the existence of three particle families points to some underlying structure as well.

- Grand Unification: The strengths of the three forces contained within the SM depend on the energy scale, as shown in Fig. 2.4. In addition, the strength also depend on the types of particles that interact with the appropriate force. If all three were to cross at a single point, it could be an indication that each one is a separate piece of a larger force. The combination of the three forces is known as grand unification theory (GUT). 


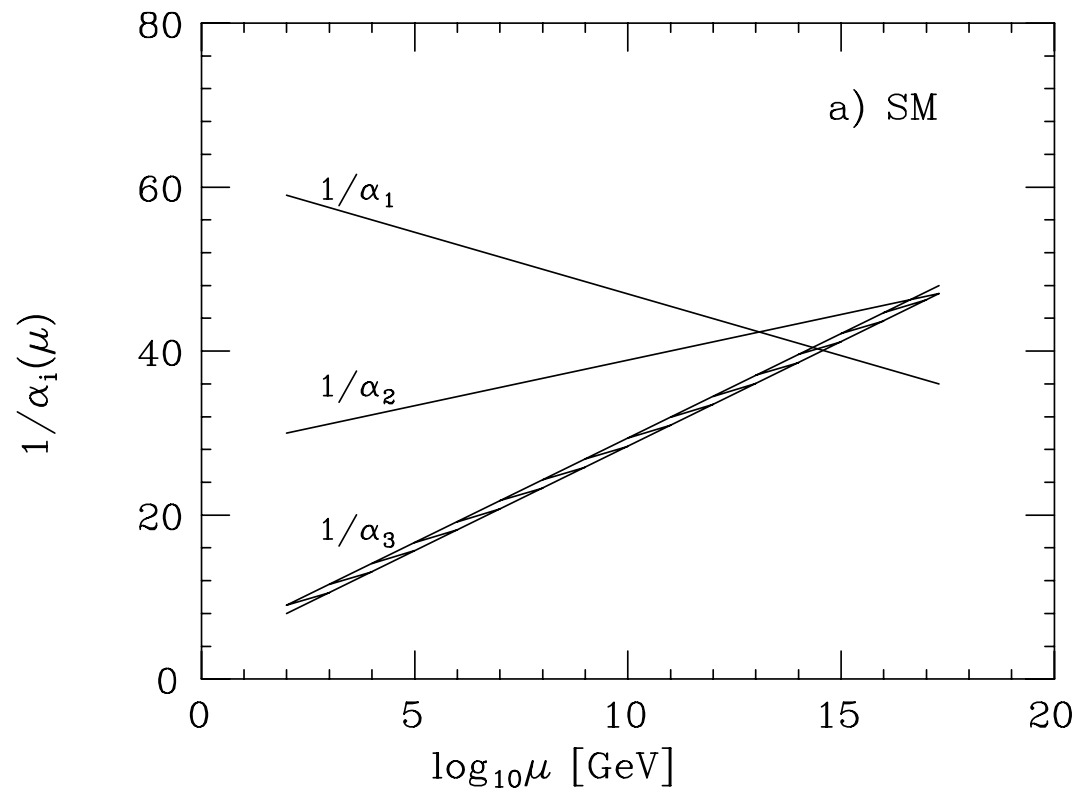

Figure 2.4: Strength of each SM force $\left(\alpha_{i}\right)$ as a function of energy scale $(\mu)$.

With only the particles included in the SM, the forces come close but do not all intersect at the same point. This near intersection could indicate that there are undiscovered particles which affect how the coupling strengths of the three interactions and cause them to intersect.

\subsection{Supersymmetry}

Of all the proposed theories that predict new physics beyond the SM, Supersymmetry (SUSY) [21] is one of the most sought after. In this Section, I will describe what SUSY is, its implications, its motivation, and how it can be used to predict new particles that may be discovered by current particle physics detectors.

SUSY is a theoretical symmetry relating fermions to bosons. More precisely, it describes a transformation that changes the spin of a particle by $\frac{1}{2}$ unit. For 
example, if an electron undergoes a supersymmetric transformation, it would result in a particle with exactly the same properties, except that its spin would now be 0 instead of $\frac{1}{2}$. Using this principle, SUSY predicts a new particle, known as a superpartner, for every (non Higgs) particle in the SM except with a spin differing by $\frac{1}{2}$. In the minimal supersymmetric model (MSSM), to have a selfconsistent supersymmetric theory, there must also be a total of four Higgs bosons, two neutral and two charged, instead of a single Higgs proposed by the SM. The MSSM has the least number of particles, but there are other SUSY variations that make different assumptions.

A direct result of having a fermionic superpartner for every boson in the SM and a bosonic superpartner for every fermion in the SM, is the exact cancellation of the hierarchy problem. With the addition of the superpartners, there are new interactions with the Higgs that also lead to the same dependence, but with the opposite sign. By adding the two terms together, they cancel and therefore the hierarchy problem vanishes, as shown in Fig. 2.5.

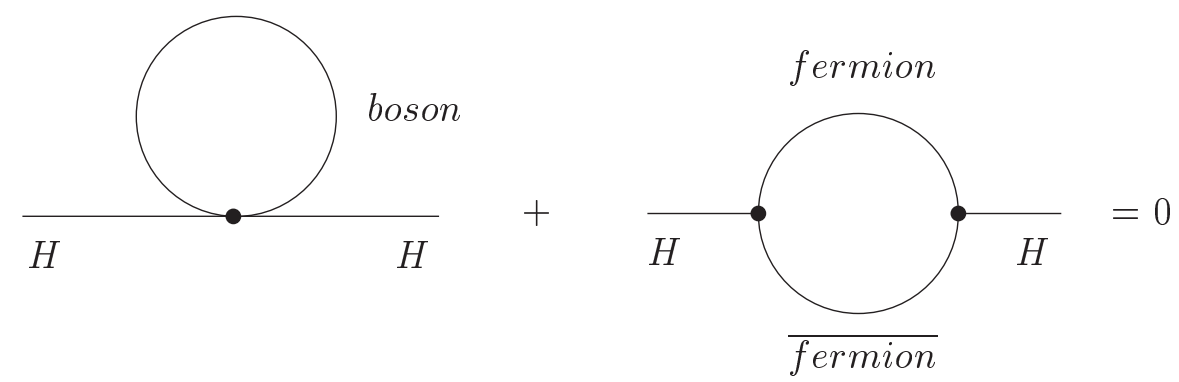

Figure 2.5: Cancellation of divergent corrections to the Higgs mass.

\subsubsection{Supersymmetry Breaking}

While SUSY provides an elegant solution to the hierarchy problem, to date no superpartners have been observed. This is not a trivial problem since most par- 
ticles with small masses $\left(<100 \mathrm{GeV} / c^{2}\right)$ are easily detectable at current particle accelerators. Either SUSY does not exist in nature, or there must be an explanation that results in SUSY particles being much heavier than their SM partners. This can occur if SUSY is a broken symmetry, where only superpartners and not SM particles acquire mass through the breaking mechanism. If SUSY is broken, then two of the implications are: the mass of each superpartner is unknown and becomes a free parameter of the theory, and the divergences which used to cancel under unbroken SUSY to solve the hierarchy problem no longer cancel exactly. As long as the SUSY breaking mechanism is "soft" [22], then the superpartner masses will be on the order of several $\mathrm{TeV}$ or less and the quadratic dependence of the Higgs mass corrections on $\Lambda$ is still avoided.

One motivation for massive superpartners is its possible contribution to the unification of the SM forces at the grand unification energy scale. With additional particles that interact via the forces of the SM, the dependence of the coupling strengths as a function of energy scale changes. Depending on the superpartner masses, the running of the SM couplings can be modified in such a way that they cross at the same energy scale, as shown in Fig. 2.6.

\subsubsection{Supersymmetric Particle Spectrum}

The particles within the MSSM are listed in Table 2.3. All superpartners are denoted by a tilde $(\sim)$ above the particle. The names of the superpartners depend on whether they are spin 0 or spin $\frac{1}{2}$. Each spin 0 superpartner is named by taking its SM partner and adding an s- to the front, for instance, the superpartner of an electron is a selectron $(\tilde{e})$. For the spin $\frac{1}{2}$ superpartners, the particles are named by adding or replacing the last syllable with an -ino. For example, the superpartner of a gluon is a gluino $(\tilde{g})$. The superpartners of the electroweak 


\begin{tabular}{|c|c|c|}
\hline SM particles & \multicolumn{2}{|c|}{ Superpartners } \\
\hline leptons (spin $\left.\frac{1}{2}\right)$ & \multicolumn{2}{|c|}{ sleptons $(\operatorname{spin} 0)$} \\
\hline$e, \mu, \tau$ & \multicolumn{2}{|c|}{$\tilde{e}, \tilde{\mu}, \tilde{\tau}$} \\
\hline$\nu_{e}, \nu_{\mu}, \nu_{\tau}$ & \multicolumn{2}{|c|}{$\tilde{\nu}_{e}, \tilde{\nu}_{\mu}, \tilde{\nu}_{\tau}$} \\
\hline quarks ( $\left.\operatorname{spin} \frac{1}{2}\right)$ & \multicolumn{2}{|c|}{ squarks (spin 0) } \\
\hline$u, c, t$ & \multicolumn{2}{|c|}{$\tilde{u}, \tilde{c}, \tilde{t}$} \\
\hline$d, s, b$ & \multicolumn{2}{|c|}{$\tilde{d}, \tilde{s}, \tilde{b}$} \\
\hline neutral bosons $(\operatorname{spin} 0,1)$ & \multicolumn{2}{|c|}{ gluino, neutralinos $\left(\operatorname{spin} \frac{1}{2}\right)$} \\
\hline$g$ & \multicolumn{2}{|c|}{$\tilde{g}$} \\
\hline$\gamma$ & $\tilde{\gamma}$ & \multirow{3}{*}{$\Rightarrow \tilde{\chi}_{1-4}^{0}$} \\
\hline$Z$ & $\tilde{Z}$ & \\
\hline$H_{u}^{0}, H_{d}^{0}$ & $\tilde{H}_{u}^{0}, \tilde{H}_{d}^{0}$ & \\
\hline charged bosons $(\operatorname{spin} 0,1)$ & \multicolumn{2}{|c|}{$\operatorname{charginos}\left(\operatorname{spin} \frac{1}{2}\right)$} \\
\hline$W^{ \pm}$ & $\tilde{W}^{ \pm}$ & \multirow{2}{*}{$\Rightarrow \tilde{\chi}_{1-2}^{ \pm}$} \\
\hline$H^{ \pm}$ & $\tilde{H}^{ \pm}$ & \\
\hline
\end{tabular}

Table 2.3: SM particles (left) and their corresponding superpartners in the MSSM (right).

gauge bosons and Higgs bosons mix to produce four neutral mass particles, or neutralinos $\left(\tilde{\chi}_{1-4}^{0}\right)$ and two charged particles, known as charginos $\left(\tilde{\chi}_{1-2}^{ \pm}\right)$. In general, the superpartners of fermions in right- and left-handed helicity states do not have to have the same mass, i.e. $\mathrm{M}\left(\tilde{e}_{R}\right) \neq \mathrm{M}\left(\tilde{e}_{L}\right)$ and can mix with each other, i.e. $\tilde{t}_{R, L} \rightarrow \tilde{t}_{1,2}$. 


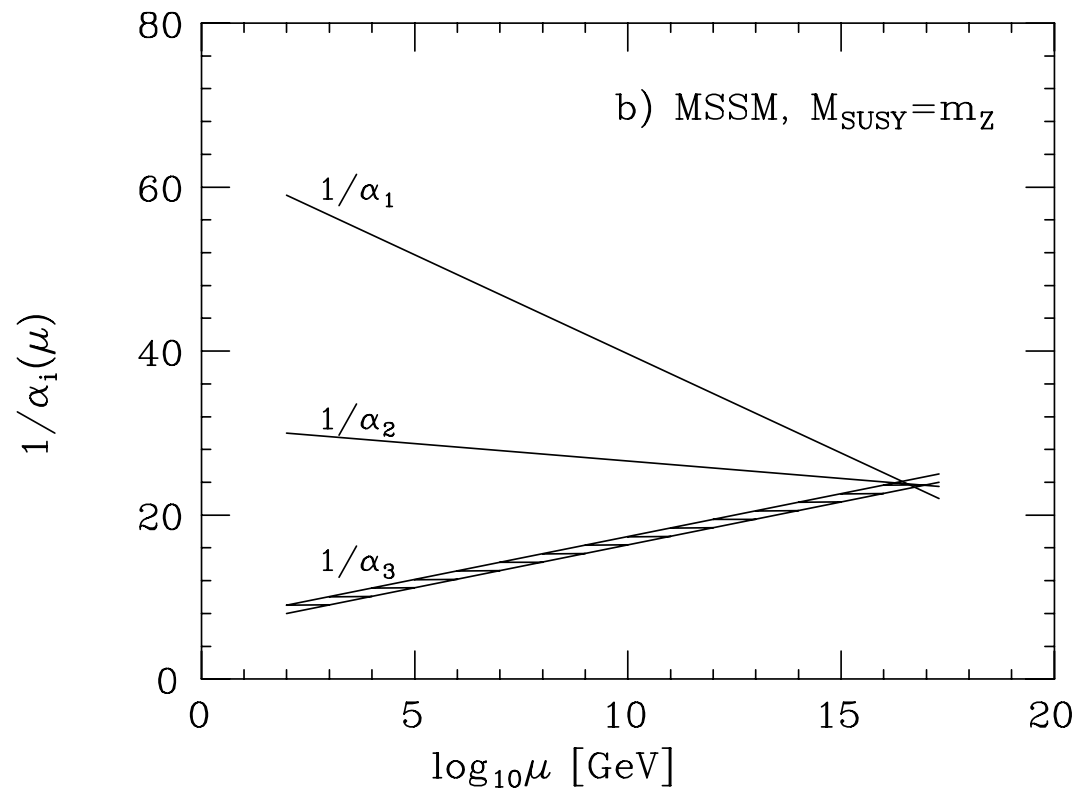

Figure 2.6: Strength of each SM force $\left(\alpha_{i}\right)$ as a function of energy scale $(\mu)$ after including supersymmetric particles.

\subsubsection{Minimal Supergravity}

In the MSSM there are over 100 free parameters, since the superpartner masses and couplings, mass mixing angles, and details of SUSY breaking are introduced by hand. Three of the parameters, $M_{1}, M_{2}$, and $M_{3}$, specify the gaugino masses associated with the $\mathrm{U}(1), \mathrm{SU}(2)$, and $\mathrm{SU}(3)$ subgroups at the breaking scale. There are five parameters corresponding to superpartner masses of the SM fermions. The parameter $\tan \beta$ specifies the ratio of the two Higgs fields, $\mathrm{H}_{u}$ and $\mathrm{H}_{d}$. In addition, there are "A-parameters" which describe the trilinear couplings: Higgs-squark-squark and Higgs-slepton-slepton. There is also the higgsino mass parameter $(\mu)^{4}, \mu$, which appears in Eq. 2.4. The remaining parameters can be found in [23].

\footnotetext{
${ }^{4}$ Unfortunately, the same symbol, $\mu$, is used for both the muon particle and the higgsino mass parameter.
} 
This analysis will focus on one framework known as minimal supergravity [24] (mSUGRA), since it attempts to connect gravity with the other forces of the SM and there are only 5 free parameters. In mSUGRA it is assumed that SUSY is a local symmetry while MSSM considers SUSY to be global. Just like in local gauge invariance, to go from a global to a local symmetry, new particles must be introduced. In the mSUGRA framework, these fields correspond to the graviton, the mediator of the gravitational force, and its superpartner, the gravitino. In mSUGRA, SUSY is spontaneously broken, thus giving the gravitino additional mass using the same Higgs mechanism that is responsible for breaking the electroweak symmetry and giving mass to the weak bosons. Additionally, mSUGRA provides a dynamical explanation for electroweak symmetry breaking.

In the mSUGRA framework the parameters are constrained such that only five are needed to determine all SUSY masses and coupling strengths:

- $M_{1 / 2}$ - the universal gaugino mass

- $M_{0}$ - the universal scalar mass

- $\tan \beta$ - the ratio of the Higgs vacuum expectation value

- $A_{0}$ - the universal trilinear coupling

- $\operatorname{sign}(\mu)$ - the sign, either positive or negative, of the higgsino mass parameter

These parameters specify all the information at the Planck scale: $M_{1 / 2}$ unifies the gaugino mass parameters at the Planck scale, $M_{0}$ unifies the five sfermion mass parameters at the Planck scale, and $A_{0}$ unifies the A-parameters at the Planck scale. The parameters of $\tan \beta$ and $\mu$ are universal for all SUSY theories regardless of the breaking mechanism, while only the sign of $\mu$ is important for mSUGRA. From these initial conditions, superpartner masses at the electroweak scale can 
be determined using the renormalization group equations. Fig. 2.7 shows the superpartner masses for one particular choice of mSUGRA parameters.

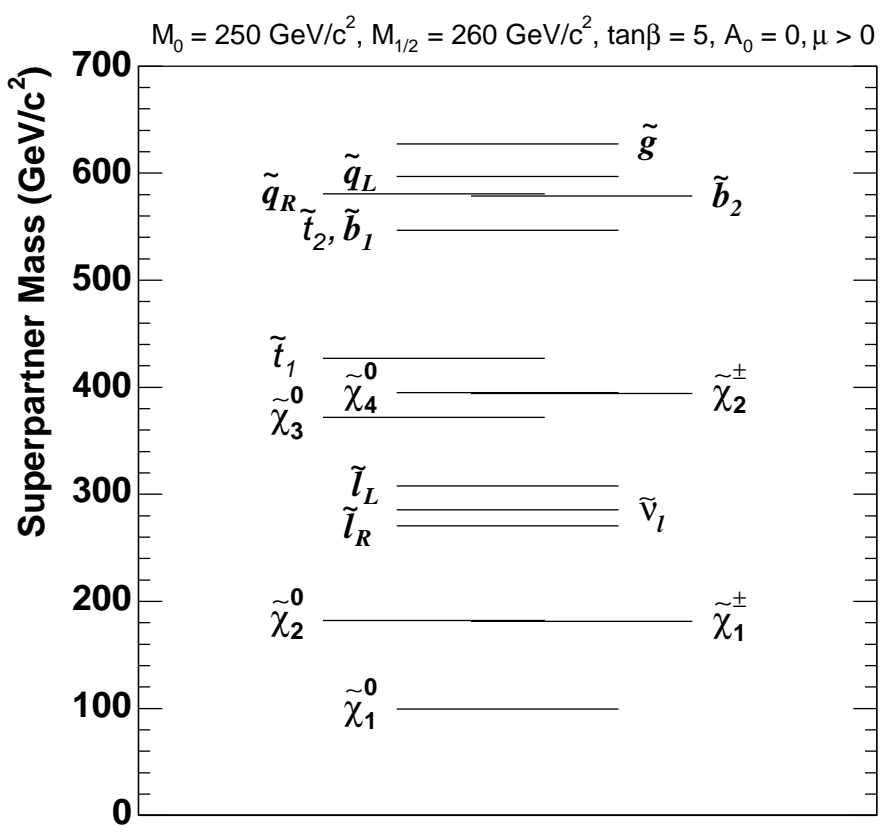

Figure 2.7: Superpartner masses for a particular choice of mSUGRA parameters.

\subsubsection{R-parity}

The quantum number R-parity is defined by Equation 2.5:

$$
R_{p}=(-1)^{[3(B-L)+2 S]}
$$

where $\mathrm{B}$ is the baryon number, $\mathrm{L}$ is the lepton number, and $\mathrm{S}$ is the particle spin. $\mathrm{B}$ and $\mathrm{L}$ are defined such that all baryons have a value of $\mathrm{B}=+1$ and all leptons have a value of $\mathrm{L}=+1$. For antiparticles, the signs of $\mathrm{B}$ and $\mathrm{L}$ become negative. $R_{p}$ is introduced since its value is +1 for every SM particle and -1 for all the superpartners. As mentioned in Section 2.7, every symmetry has a 
corresponding conserved quantity. If $R_{p}$ is this conserved quantity then $\mathrm{B}-\mathrm{L}$ would also be conserved. Although the reasons behind it are not understood, both B and L are conserved in the SM. As a consequence the proton is stable, since if $\mathrm{B}$ and $\mathrm{L}$ are violated, then it could decay into a positron and a pi-zero $\left(p \rightarrow e^{+}+\pi^{0}\right)$. With $R_{p}$ conservation, the proton is guaranteed to be stable from supersymmetric decays as well. For all interactions, if we multiply the $R_{p}$ quantum numbers of all the particles in the initial state it must equal the product of the $R_{p}$ quantum numbers of all the particles in the final state. This restricts SM particle/superpartner interactions such that superpartners must be pair produced and the lightest supersymmetric particle (LSP) is stable. In this scenario, the LSP is an excellent candidate for dark matter.

\subsubsection{R-parity Violation}

While $R_{p}$ is motivated by dark matter and proton stability, there is no guarantee that if SUSY exists $R_{p}$ will be conserved. There are three terms within the general superpotential W [25], which describes specific ways in which $R_{p}$ may be violated:

$$
W=\lambda_{i j k} L_{i} L_{j} \bar{E}_{k}+\lambda_{i j k}^{\prime} L_{i} Q_{j} \bar{D}_{k}+\lambda_{i j k}^{\prime \prime} \bar{U}_{i} \bar{D}_{j} \bar{D}_{k},
$$

where $\lambda_{i j k}, \lambda_{i j k}^{\prime}$, and $\lambda_{i j k}^{\prime \prime}$ denote the coupling strengths; $i, j$, and $k$ refer to the family indices ranging from 1-3; $L_{i}$ and $Q_{i}$ are the left-handed lepton and quark doublet superfields and $\bar{E}_{i}, \bar{D}_{i}$, and $\bar{U}_{i}$ are the right-handed singlet superfields for charged leptons, down, and up type quarks, respectively. In quantum field theory, the superfields describe both the SM and SUSY particles.

The first two terms in Equation 2.6 violate lepton number and the third violates baryon number. For the proton to remain stable, either $\lambda^{\prime}$ or $\lambda^{\prime \prime}$ must be 0 . Additional constraints on the values of the coupling strengths will be discussed 
in the next section.

\subsubsection{4-Lepton Signature}

Since there are many new free parameters associated with the masses of the predicted superpartners and their couplings, there currently is no absolute test whether SUSY is a symmetry of nature. Typically, searches for new physics look for an excess of events with a specific signature, as compared to the SM. Since production cross sections of SUSY particles are expected to be orders of magnitude smaller than for SM processes at the Tevatron, a signature is desired which reduces the SM background while preserving new physics signals. Such methods include searching for leptons with large momentum transverse to the beam axis $\left(p_{T}\right)$, like-sign leptons, or signatures with transverse missing energy $\left(\mathscr{E}_{T}\right)$, where $\mathscr{E}_{T}$ is a result of particles that rarely interact with other forms of matter such as neutrinos or the LSP in $R_{p}$ conserving SUSY, making them nearly impossible to detect. This analysis describes a signature-based search for proton-antiproton collisions producing a final state of four or more leptons inconsistent with the SM. There are several new physics models that produce this signature including $\ell_{p}$ SUSY (Sec. 2.11.7), doubly-charged Higgs pair production (Sec. 2.12.1), and non-minimal supersymmetric models (Sec. 2.12.2). This thesis focuses on the $\not_{p}$ SUSY model while attempting to maintain sensitivity to as many new physics models as possible.

\subsubsection{R-parity Violating Supersymmetry}

Of the three terms within Eq. 2.6, only the first term may lead exclusively to a final state of four or more leptons when superpartners are produced in protonantiproton collisions. Exactly how this final state is produced will be described 
later in the section. Therefore I investigate a scenario in which $\lambda_{i j k} \neq 0$ while $\lambda_{i j k}^{\prime}=\lambda_{i j k}^{\prime \prime}=0$. With baryon number conservation, there is no possibility for proton decay and superpartners must be pair produced at hadron colliders. In addition, the search is restricted to the first two lepton families since tau leptons are less efficiently detected. For this reason, this analysis only considers $\lambda_{i j k}$ terms corresponding to the first two lepton families $\left(\lambda_{121}, \lambda_{122}\right)$.

Since $\ell_{p}$ SUSY allows new interactions between particles and superpartners, there may be existing experimental results which limit the possible values of $\lambda_{12 k}$. For example, the muon can decay either through the $\lambda_{121}$ or $\lambda_{122}$ couplings into an electron and two neutrinos, shown in Fig. 2.8. If $\lambda_{12 k}$ is higher than the value specified in Eq. 2.7 then it would result in different muon decay properties from what is experimentally observed:

$$
\lambda_{12 k}<0.05 \times \frac{\mathrm{M}(\tilde{e}, \tilde{\mu})}{100 \mathrm{GeV} / c^{2}} \approx 0.14
$$

This constraint is known as charged current universality in lepton decay. There is also a lower bound to our sensitivity on $\lambda_{12 k}$ due to the lifetime of the LSP, in Eq. 2.8, where $\alpha_{E M}$ is the coupling strength of the electromagnetic interaction:

$$
\Gamma_{\tilde{\gamma}}=\frac{\alpha_{E M} \lambda_{i j k}^{2} \mathrm{M}(L S P)^{5}}{128 \pi^{2} \mathrm{M}(\tilde{e}, \tilde{\mu})^{4}}
$$

If $\lambda_{12 k} \lesssim 3 \times 10^{-3}$ then the LSP will decay far from the proton-antiproton interaction region. Putting the two limits together, the range of $\lambda_{12 k}$ values relevant to our search is given by:

$$
3 \times 10^{-3} \lesssim \lambda_{12 k} \lesssim 0.14
$$

Using PROSPINO2 [26], cross sections for different types of superpartner pair production are investigated. See Table 2.4 for a listing of various particle production cross sections for a particular choice in the mSUGRA parameter space. 

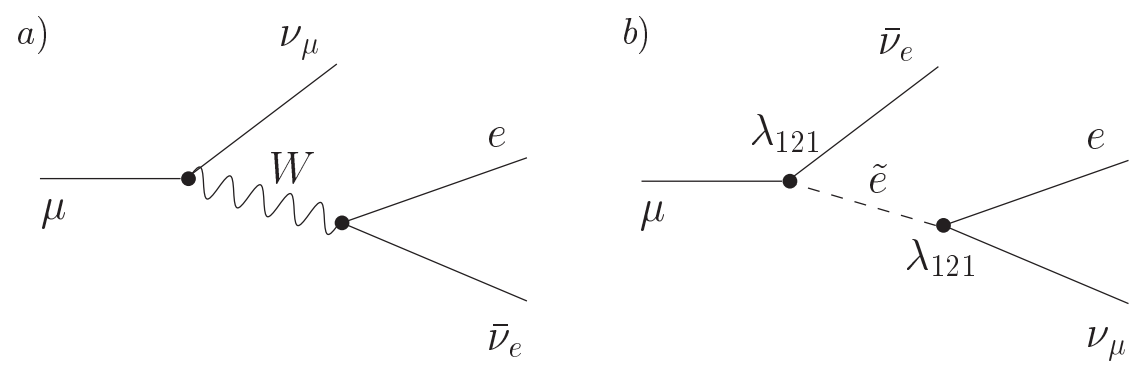

Figure 2.8: Muon decay through a) the weak force and b) via an $\mathbb{R}_{p}$ violating vertex. Muon decay is also possible with $\lambda_{122}$ by replacing the $\tilde{e}$ with a $\tilde{\mu}$ in the second diagram.

Chargino-neutralino production $\left(q \bar{q} \rightarrow \tilde{\chi}_{2}^{0} \tilde{\chi}_{1}^{ \pm}\right)$and chargino pair production $\left(q \bar{q}^{\prime} \rightarrow\right.$ $\left.\tilde{\chi}_{1}^{ \pm} \tilde{\chi}_{1}^{\mp}\right)$ are the dominant processes, and are produced either through quarkantiquark annihilation or through squark exchange, as shown in Fig. 2.9. Charginos and neutralinos then decay into the LSP, which is the $\tilde{\chi}_{1}^{0}$ for all the mSUGRA parameters considered, through the Feynman diagrams in Fig. 2.10. It is possible for the $\tilde{\chi}_{2}^{0}$ and $\tilde{\chi}_{1}^{ \pm}$particles to decay via the $\lambda_{12 k}$ interaction term, although the allowed values listed in Eq. 2.9 are sufficiently small so that these processes are unlikely and can be neglected.

As a result of the $\tilde{\chi}_{2}^{0}$ and $\tilde{\chi}_{1}^{ \pm}$"cascade" decays, there will be two $\tilde{\chi}_{1}^{0}$ particles with additional leptons and jets, depending on the details of the decays. The LSP can only decay through the allowed $k_{p}$ interaction into two opposite sign leptons (either one may be an electron or a muon) plus a neutrino, shown in Fig. 2.11. Therefore there will be at least four leptons in the event. For the $\lambda_{121}\left(\lambda_{122}\right)$ coupling at least two of the four will be electrons (muons). This signature of four or more leptons, will also be referred to as the signal throughout this thesis. The signal of four or more leptons provides an excellent method of searching for $k_{p}$ SUSY, since there are very few mechanisms within the SM that result in the 


\begin{tabular}{lc}
\hline Process & Cross Section (fb) \\
\hline$p \bar{p} \rightarrow \tilde{\chi}_{2}^{0} \tilde{\chi}_{1}^{ \pm}+\mathrm{X}$ & 73.5 \\
$p \bar{p} \rightarrow \tilde{\chi}_{1}^{ \pm} \tilde{\chi}_{1}^{\mp}+\mathrm{X}$ & 54.5 \\
$p \bar{p} \rightarrow \tilde{\chi}_{1}^{0} \tilde{\chi}_{1}^{ \pm}+\mathrm{X}$ & 3.58 \\
$p \bar{p} \rightarrow \tilde{\chi}_{1}^{0} \tilde{\chi}_{1}^{0}+\mathrm{X}$ & 0.97 \\
$p \bar{p} \rightarrow \tilde{\chi}_{2}^{0} \tilde{\chi}_{2}^{0}+\mathrm{X}$ & 0.91 \\
$p \bar{p} \rightarrow \tilde{\tau}_{1} \tilde{\tau}_{1}+\mathrm{X}$ & 0.07 \\
$p \bar{p} \rightarrow \tilde{\nu}_{e} \tilde{\nu}_{e}+\mathrm{X}$ & 0.06 \\
$p \bar{p} \rightarrow \tilde{\chi}_{1}^{0} \tilde{q}+\mathrm{X}$ & 0.05 \\
$p \bar{p} \rightarrow \tilde{l}_{L} \tilde{l}_{L}+\mathrm{X}$ & 0.04 \\
\hline
\end{tabular}

Table 2.4: Production cross sections of superpartner pairs in proton-antiproton collisions at $\sqrt{s}=1.96 \mathrm{TeV}$ for $M_{0}=250, M_{1 / 2}=260, \tan \beta=5, A_{0}=0$, and $\mu>0$. Processes with smaller cross sections are not listed.

same signature (see Section 5.3).

Previous analyses have searched for this type of $k_{p}$ SUSY model using a signature of four leptons [27] or three leptons plus $E_{T}$ [28]. So far, all searches for $k_{p}$ SUSY, including only lepton number violation, have observed results consistent with the SM. In order for this theory to remain valid while being in agreement with previous results, the superpartners must be heavier than a certain value. As of this writing, current production cross section and mass limits on the LSP are listed in Table 2.5 assuming $\lambda_{121}$ is the only $\not_{p}$ coupling term and in Table 2.6 assuming $\lambda_{122}$ is the only $h_{p}$ coupling term. 

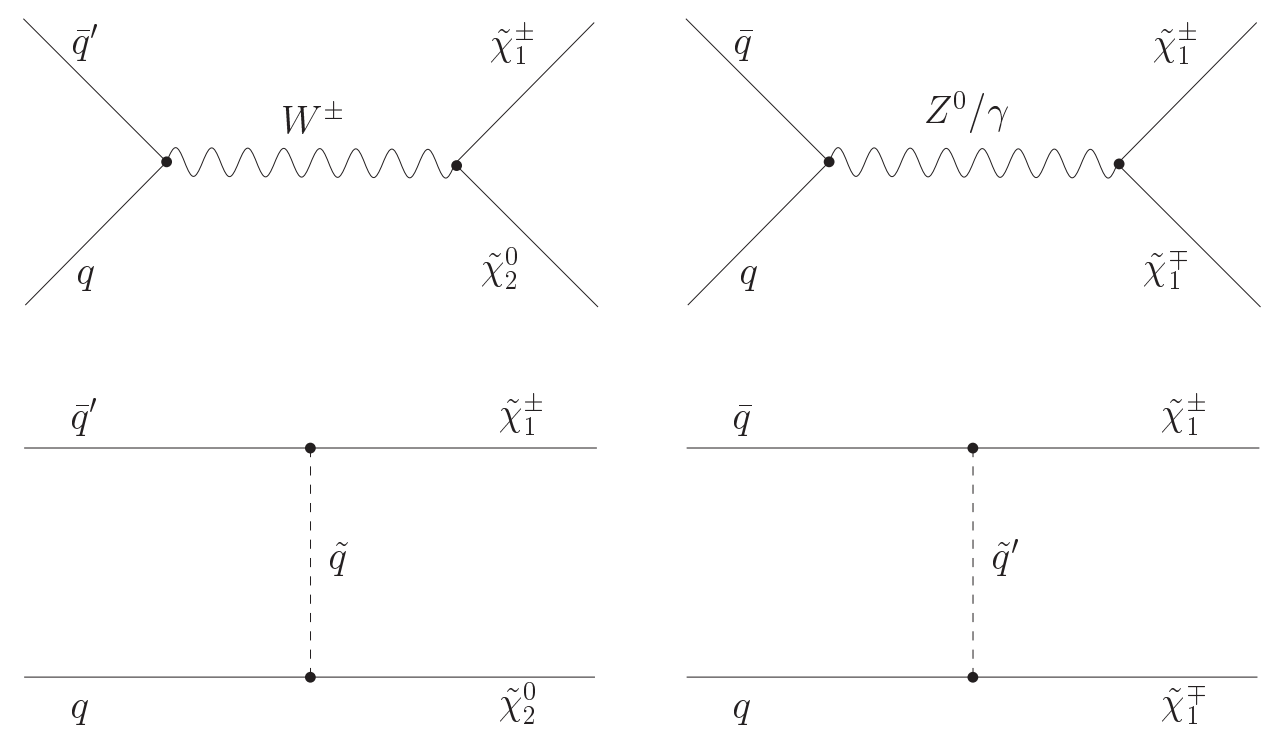

Figure 2.9: Chargino-neutralino and chargino pair production in proton-antiproton collisions.

\subsubsection{Dependence on Minimal Supergravity Parameters}

The mSUGRA parameters affect the masses and coupling strengths between the superpartners. To study the impact of varying the parameters, superpartner masses and decay modes were determined for values of $M_{0}$ between 100-400 $\mathrm{GeV} / c^{2}, M_{1 / 2}$ between $100-400 \mathrm{GeV} / c^{2}, \tan \beta$ between $5-50, A_{0}$ between -400 and 400 , and both signs of the higgsino mass parameter, using ISAJET [30]. In this analysis, the most important parameter is $M_{1 / 2}$, which is related to the masses of all the superpartners with spin $\frac{1}{2}$ including the $\tilde{\chi}_{1}^{0}, \tilde{\chi}_{2}^{0}$, and $\tilde{\chi}_{1}^{ \pm}$. As $M_{1 / 2}$ increases, so do the masses of the charginos and neutralinos, shown in Fig. 2.12, causing the pair production cross sections to decrease. Since the $\tilde{\chi}_{1}^{0}$ decays directly into two charged leptons and a neutrino, higher values of $M_{1 / 2}$ also result in leptons with larger energies that are easier to detect. The chargino and neutralino masses also depend on the sign of $\mu$ to a much lesser degree. These masses tend to be 

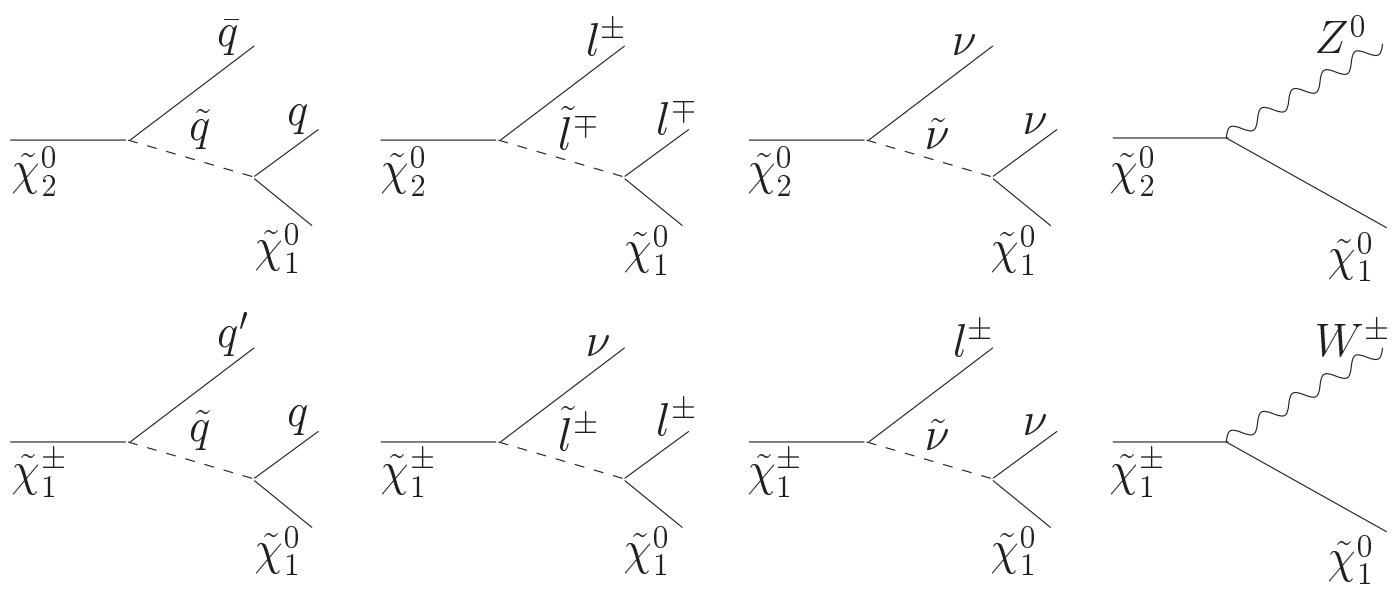

Figure 2.10: "Cascade" decays of neutralinos (top) and charginos (bottom).

higher for $\mu<0$ scenarios than in $\mu>0$ scenarios when all other mSUGRA parameters are kept constant. The $\tilde{\chi}_{1}^{0}$ mass dependence on $\tan \beta$ is shown in Fig. 2.13. For values of $\tan \beta \lesssim 2$ or $\gtrsim 50$, mSUGRA predicts unphysical results such as particles traveling faster than the speed of light (tachyons). $M_{0}$ and $A_{0}$ have very little affect on the chargino and neutralino masses. The neutralino dependence on $M_{0}$ is shown in Fig. 2.14 and the dependence on $A_{0}$ is shown in Fig. 2.15. In $\not_{p}$ SUSY, there is no strong motivation to pick a certain value of $M_{0}$ or $A_{0}$. To avoid reintroducing the hierarchy problems, squark and slepton masses should be less than several $\mathrm{TeV} / c^{2}$, which motivates a value of $M_{0}$ to be less than several $\mathrm{TeV} / c^{2}$ as well. The selected ranges of $M_{0}$ and $A_{0}$ were chosen so that the dependence on $\tilde{\chi}_{1}^{0}$ could be determined and to be centered around the values used in previous analyses.

In addition to changing the superpartner masses, the mSUGRA parameters can also affect the "cascade" decays of the charginos and neutralinos. As shown in Fig. ??, there are several modes for these decays to occur, while only some of them produce charged leptons. For example, at small $\tan \beta(\sim 5), \tilde{\chi}_{2}^{0}$ particles 
a)
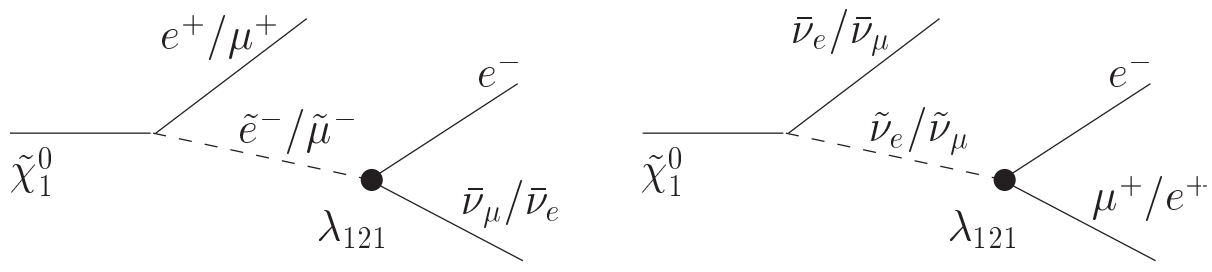

b)
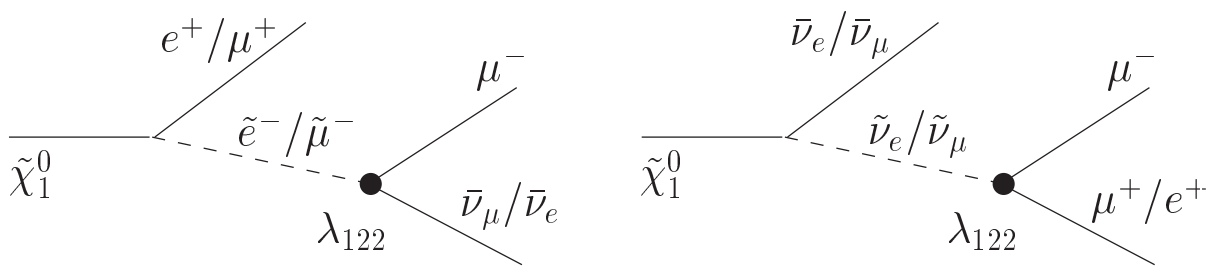

Figure 2.11: Two possible Feynman diagrams for $\not R_{p}$ SUSY decay of the LSP via each of a) the $\lambda_{121}$ coupling, and b) the $\lambda_{122}$ coupling.

primarily decay through the quark/squark diagram while at large $\tan \beta(\sim 40)$, $\tilde{\chi}_{2}^{0}$ particles often decay into tau leptons, which can then decay into hadrons, electrons, or muons. When the branching ratio into charged leptons increases, there is a greater probability of detecting a certain number of them, and therefore a better chance of discovering $\not_{p}$ SUSY. In general, the leptons resulting from "cascade" decays are less energetic than the leptons which are produced from the LSP decay. For this reason, the number of charged leptons produced from "cascade" decays has a much lesser impact on the final result than the mass of the LSP.

For the investigated region of parameter space, the $\tilde{\chi}_{1}^{0}$ was always the LSP. Therefore the signature of four or more charged leptons is independent of the values of mSUGRA parameters. 


\begin{tabular}{|c|c|c|c|c|c|}
\hline Experiment & $\mathcal{L}\left(\mathrm{pb}^{-1}\right)$ & $\sigma \times \mathrm{BR}$ limit $(\mathrm{pb})$ & $\mathrm{M}\left(\tilde{\chi}_{1}^{0}\right)\left(\mathrm{GeV} / c^{2}\right)$ & $\tan \beta$ & $\mu$ \\
\hline CDF Run I & 87.5 & 0.56 & 65 & 2 & $<0$ \\
\hline D0 Run II & 360 & 0.116 & 117 & 5 & $<0$ \\
\hline D0 Run II & 360 & 0.115 & 119 & 5 & $>0$ \\
\hline
\end{tabular}

Table 2.5: Limits on $\lambda_{121}$ from previous $\not R_{p}$ SUSY searches.

\begin{tabular}{|c|c|c|c|c|c|}
\hline Experiment & $\mathcal{L}\left(\mathrm{pb}^{-1}\right)$ & $\sigma \times \mathrm{BR}$ limit $(\mathrm{pb})$ & $\mathrm{M}\left(\tilde{\chi}_{1}^{0}\right)\left(\mathrm{GeV} / c^{2}\right)$ & $\tan \beta$ & $\mu$ \\
\hline D0 Run II & 360 & $\sim 0.239$ & 115 & 5 & $<0$ \\
\hline D0 Run II & 360 & $\sim 0.190$ & 118 & 5 & $>0$ \\
\hline
\end{tabular}

Table 2.6: Limits on $\lambda_{122}$ from previous $\not R_{p}$ SUSY searches.

\subsection{Other Theories Resulting in 4 or More Leptons}

\subsubsection{Doubly-Charged Higgs Pair Production}

In supersymmetric models with left-right symmetry [31], there are additional Higgs bosons with twice as much electric charge as an electron, known as doublycharged Higgs $\left(H^{ \pm \pm}\right)$. In this model, the $H^{ \pm \pm}$primarily decays into two charged leptons when $m_{H^{ \pm \pm}}<2 m_{H^{ \pm}}$and $m_{H^{ \pm \pm}}<m_{W^{ \pm}}$. If a pair of doubly-charged Higgs is produced through $p \bar{p} \rightarrow Z / \gamma^{*} \rightarrow H^{++} H^{--}$, then there could be four leptons in the final state. Since the $H^{ \pm \pm}$decays directly into two charged leptons, the final state will not contain any $\mathbb{E}_{T}$ or jets, unless produced by another mechanism.

\subsubsection{Non-Minimal Supersymmetric Models}

Minimal supersymmetric models are often considered since they are the simplest, containing the least number of superpartners. In the $R_{p}$ conserving MSSM, the "golden" mode for discovery at the Tevatron is the trilepton plus missing energy 


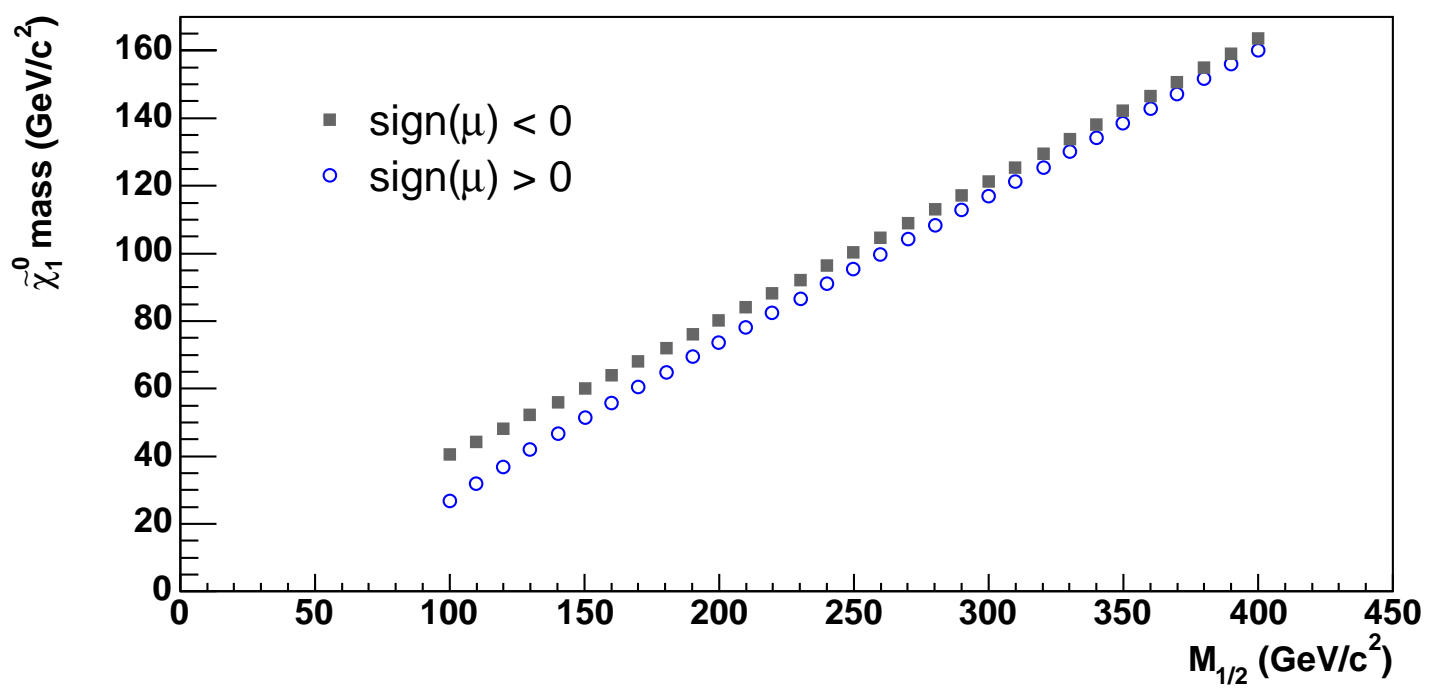

Figure 2.12: Mass of the lightest neutralino as a function of $M_{1 / 2}$ for both signs of the higgsino mass parameter $\mu, M_{0}=250 \mathrm{GeV} / c^{2}, \tan \beta=5$, and $A_{0}=0$.

signature. This final state is produced by chargino-neutralino production (see Fig. 2.9 ), where the leptons result from "cascade" decays of the $\tilde{\chi}_{2}^{0}$ and $\tilde{\chi}_{1}^{ \pm}$(see Fig. 2.10), and the $\boldsymbol{E}_{T}$ is a result of the two LSPs escaping detection. In extensions of the MSSM [32], which are referred to as non-minimal supersymmetric models (NMSSM), there are additional neutral Higgs bosons and their superpartners. As a result, there are additional neutralinos as well that may have much lighter masses than those shown in Fig. 2.7. With lighter neutralinos, the NMSSM may allow for large $\tilde{\chi}_{\geq 3}^{0} \tilde{\chi}_{1}^{ \pm}$production instead of $\tilde{\chi}_{2}^{0} \tilde{\chi}_{1}^{ \pm}$production for the MSSM. Through the "cascade" decays there may be four or more charged leptons in the final state. In some cases there is no lower mass limit on the LSP, compared to the MSSM where $\mathrm{M}\left(\tilde{\chi}_{1}^{0}\right) \gtrsim 45 \mathrm{GeV} / c^{2}$ and as a result there may be significantly less $\mathbb{E}_{T}$ in the final state. 


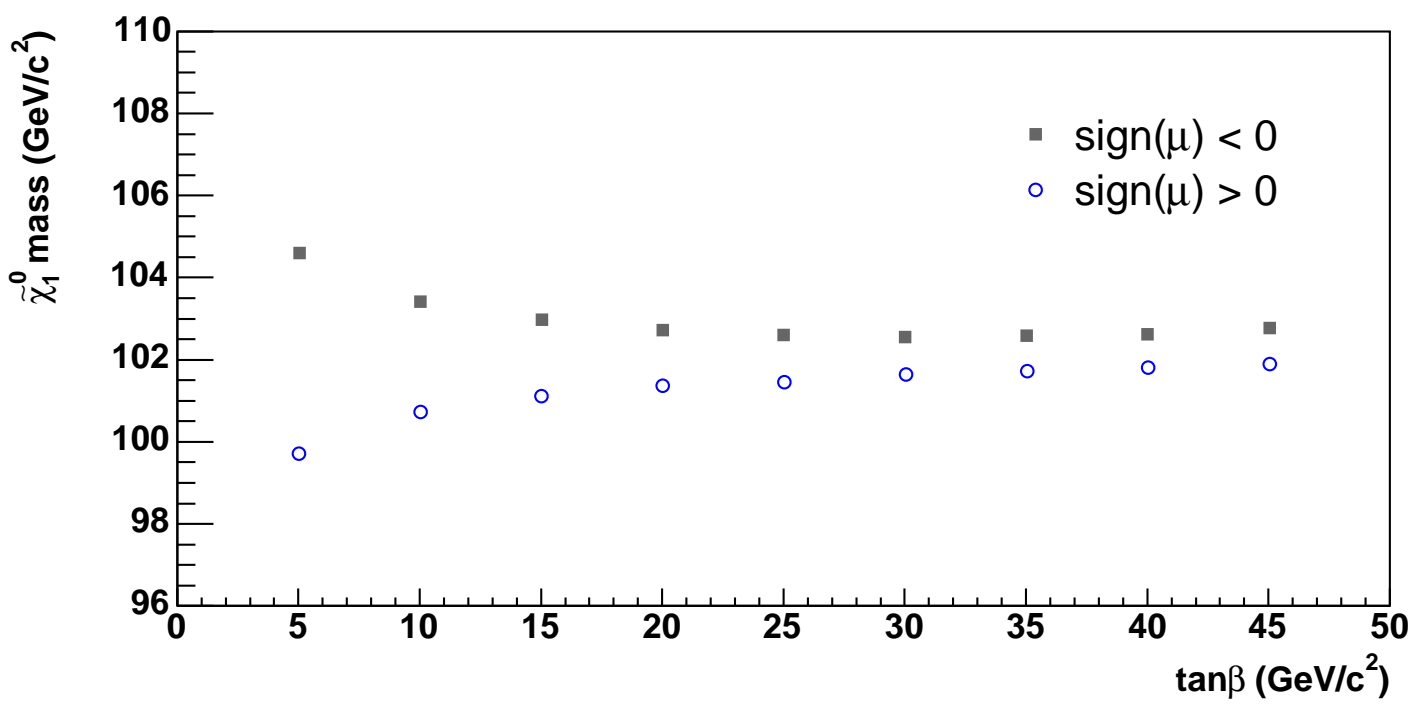

Figure 2.13: Mass of the lightest neutralino as a function of $\tan \beta$ for both signs of the higgsino mass parameter $\mu, M_{0}=250 \mathrm{GeV} / c^{2}, M_{1 / 2}=260 \mathrm{GeV} / c^{2}$, and $A_{0}=0$.

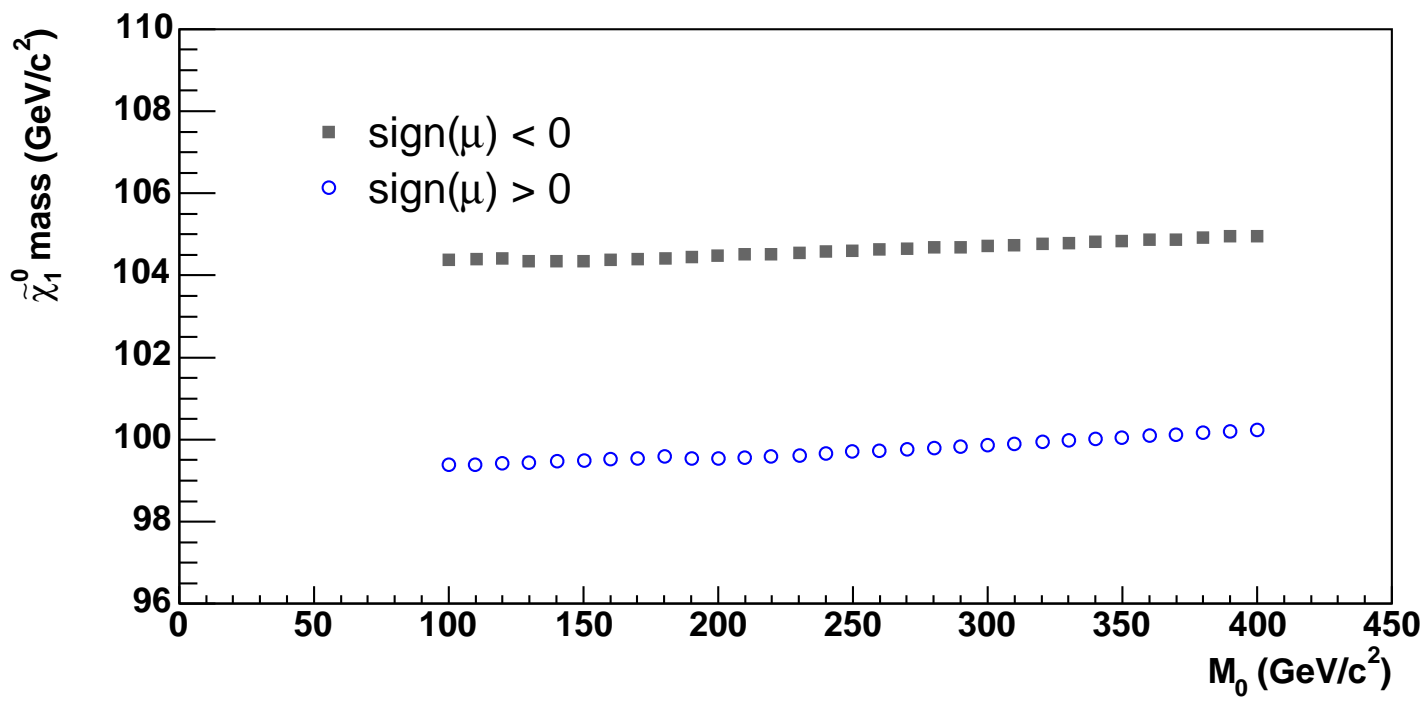

Figure 2.14: Mass of the lightest neutralino as a function of $M_{0}$ for both signs of the higgsino mass parameter $\mu, M_{1 / 2}=260 \mathrm{GeV} / c^{2}, \tan \beta=5$, and $A_{0}=0$. 


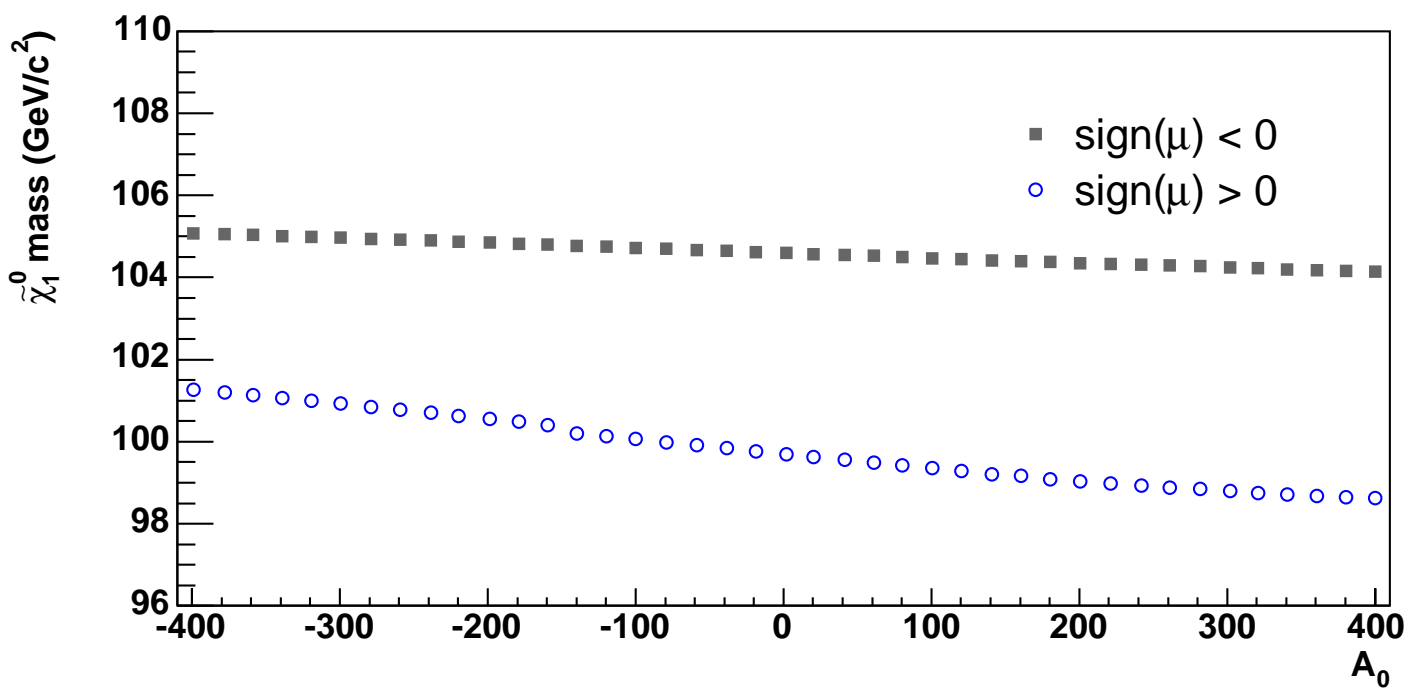

Figure 2.15: Mass of the lightest neutralino as a function of $\tan \beta$ for both signs of the higgsino mass parameter $\mu, M_{0}=250 \mathrm{GeV} / c^{2}, M_{1 / 2}=260 \mathrm{GeV} / c^{2}$, and $\tan \beta=5$. 

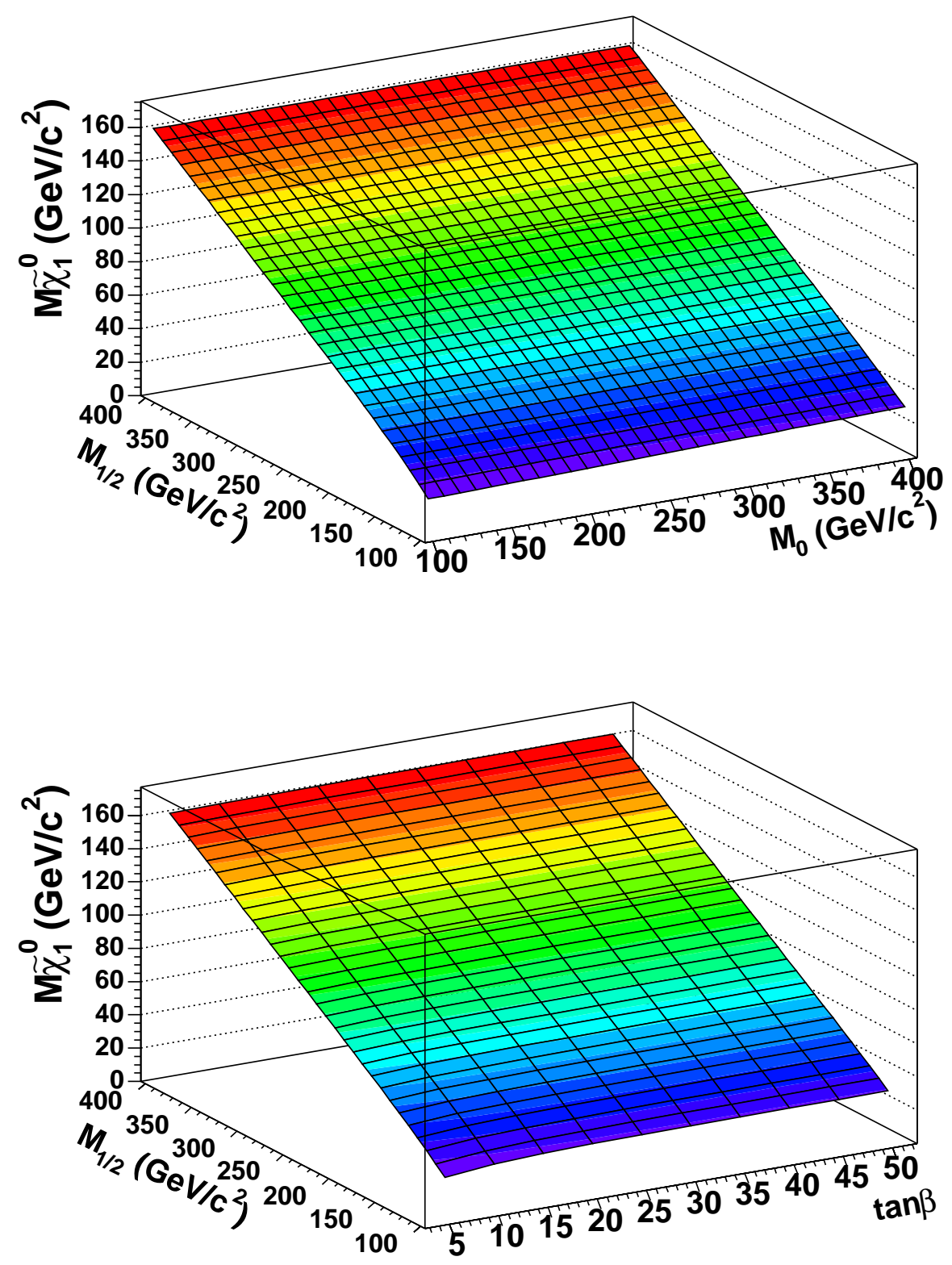

Figure 2.16: LSP mass as a function of $\mathrm{M}_{1 / 2}, \mathrm{M}_{0}$ (top), and $\tan \beta$ (bottom). The mass is highly dependent on $\mathrm{M}_{1 / 2}$ but has very little dependence on $\mathrm{M}_{0}$ or $\tan \beta$. The following parameters are used: $M_{0}=250 \mathrm{GeV} / c^{2}, \tan \beta=5, \mu>0$, and $A_{0}$ $=0$. 


\section{CHAPTER 3}

\section{Fermilab Tevatron \& CDF Detector}

High energy physics experiments require methods to produce and detect a wide range of particles with a wide range of energies. Particle energy is measured in units of electron volts $(\mathrm{eV})$ defined as the energy necessary to accelerate one electron across one volt. At the Fermi National Acceleration Laboratory (Fermilab), equal energy beams of protons and antiprotons collide with a center of mass energy of $\sqrt{s}=1.96 \mathrm{TeV}(1 \mathrm{TeV}=1$ trillion $\mathrm{eV})$. Collisions occur at two precise locations, one of which is at the center of the CDF detector, also known as the interaction point. This chapter describes the accelerator chain, proton and antiproton collisions, and CDF detector.

\subsection{Accelerator}

The purpose of the Fermilab accelerator chain is to create antiprotons and to accelerate both protons and antiprotons to an energy of $980 \mathrm{GeV}$. The accelerator chain, shown in Figure 3.1, consists of the following components: CockcroftWalton, Linac, Booster, Main Injector, Antiproton Source, Recycler, and the Tevatron.

Hydrogen atoms are taken from a bottle of compressed hydrogen gas that lasts roughly one year under normal operation. These atoms consist of an electron orbiting a proton and are electrically neutral. The atoms are then ionized to 


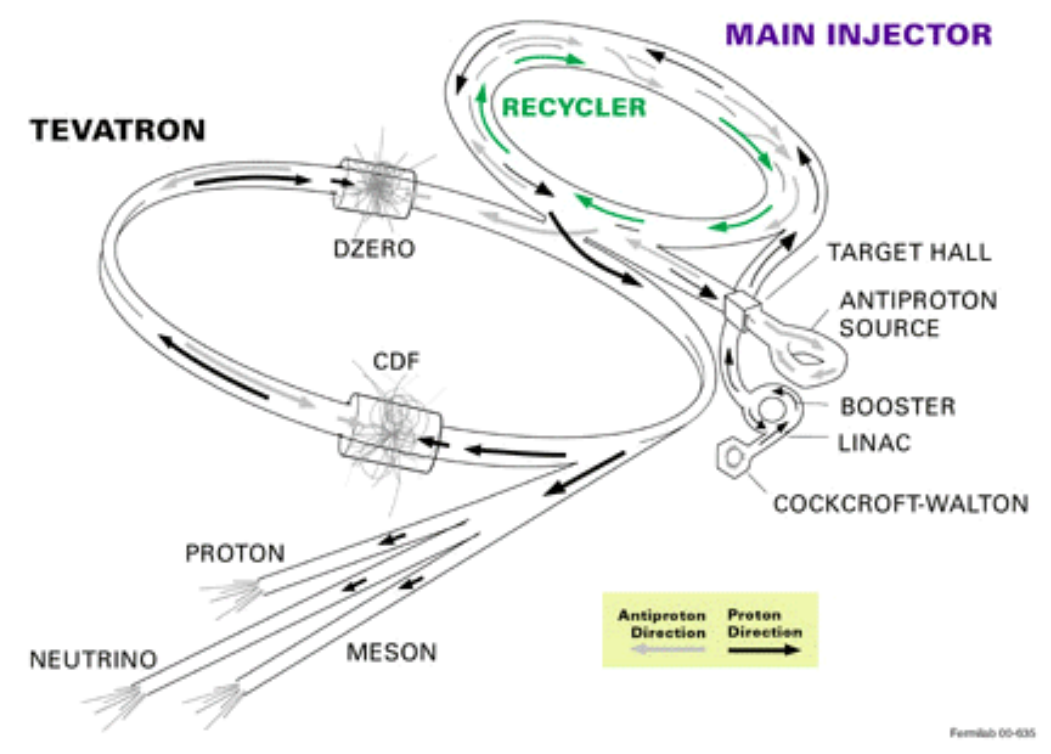

Figure 3.1: Fermilab Tevatron accelerator chain.

form $\mathrm{H}^{+}$by first stripping off the electron so that only the proton remains and placing these protons within an electric field. The protons are attracted toward a metal surface containing cesium. If the proton 'steals' two electrons from the cesium, it will attain a net negative charge, forcing them away from the surface. $\mathrm{H}^{-}$atoms are then accelerated to $750 \mathrm{keV}$ by the Cockcroft-Walton, a direct current voltage source.

The next stage is a 130 meter long linear accelerator named the Linac. The Linac accelerates $\mathrm{H}^{-}$atoms from $750 \mathrm{keV}$ to $400 \mathrm{MeV}$ through 12 radio frequency (RF) cavities. Inside each RF cavity, an alternating electric field is applied at either 201.25 or $805 \mathrm{MHz}$. In one direction the $\mathrm{H}^{-}$atoms are accelerated and in the other they are decelerated. To prevent deceleration, the atoms are shielded from the electric field within drift tubes when the electric field points in the wrong direction. A typical pulse in the Linac contains roughly 6 trillion particles. From the Linac, the $\mathrm{H}^{-}$atoms are passed through a carbon foil to strip off the two 
electrons, leaving only the proton, before entering the Booster. The Booster is a synchrotron 475 meters in circumference. A synchrotron is a circular accelerator that uses two types of magnets to synchronize the particle orbits. Dipole magnets steer the beam of protons so that they travel in a circle while quadrupole magnets focus the beam by successively squeezing it along both axes perpendicular to the direction of motion. A single RF cavity accelerates the protons once per turn as they travel around the Booster approximately 16,000 times. Protons leaving the Booster have an energy of $8 \mathrm{GeV}$.

Next in the accelerator chain is the Main Injector. It has two primary functions: to provide $120 \mathrm{GeV}$ protons for the Antiproton Source and to accelerate protons and antiprotons from 8 to $150 \mathrm{GeV}$. The Main Injector is a synchrotron $3.3 \mathrm{~km}$ in circumference. Since protons and antiprotons have opposite charge, the same magnets can be used to accelerate both particles moving in opposite directions simultaneously.

Protons from the Main Injector that are directed toward the Antiproton Source collide with a stationary nickel target. The collision between the incoming protons and the atoms within the target create many types of particles some of which will be antiprotons. For each million protons that collide into the target, only about 20 antiprotons will be collected. The outgoing particles will have a wide range of directions and energies. A lithium lens is used to focus these particles toward a pulsed magnet, separating the antiprotons from all of the other particles. The Debuncher then speeds up the slower moving antiprotons and slows down the faster ones so that they all travel at roughly the same speed. This makes it much easier to transport the antiprotons from one accelerator to another. Finally the antiprotons are sent to the Accumulator to store them until it is time to start proton-antiproton collisions. At that time, the antiprotons will 
be sent to the Main Injector.

Since antiprotons are difficult to accumulate, it is desirable to efficiently use the ones that are created. The Recycler's initial purpose was to reuse antiprotons that remain after final collisions. Currently the Recycler is used to store antiprotons from the Accumulator in order to maximize the rate that antiprotons can be stored.

The Tevatron is the final component of the acceleration process. At the Tevatron, protons are accelerated by RF cavities in the clockwise direction and antiprotons in the counterclockwise direction from 150 to $980 \mathrm{GeV}$. The protons and antiprotons are concentrated in 36 bunches each in the $6.28 \mathrm{~km}$ synchrotron. In the Tevatron, superconducting dipole magnets incorporating a niobium-titanium alloy wire and operating at a magnetic field of 4.2 Tesla are used to bend the particles. Collisions occur at two precise locations, either CDF or D0, every 396 ns. To maximize the number of collisions, magnets called low-beta quadrapoles focus the beams directly before impact. The instantaneous luminosity describes the rate at which collisions occur for two oncoming particle beams, given by:

$$
\frac{d \mathcal{L}}{d t}=R \frac{N_{p} N_{\bar{p}}}{A}
$$

where $R$ is the rate of collision, $N_{p}$ and $N_{\bar{p}}$ are the number of particles in the proton and antiproton bunches respectively, and A is the cross sectional area in which the two beams collide. The instantaneous luminosity is highest after the protons and antiprotons are initially injected into the Tevatron and brought into focus, and decreases with time due to interactions between the beams. The duration of collisions is known as a store, which ends when the beam is voluntarily or involuntarily aborted. To date, the duration of the longest store at the Tevatron is 53.75 hours occurring on July of 2006. To measure the total amount of data 


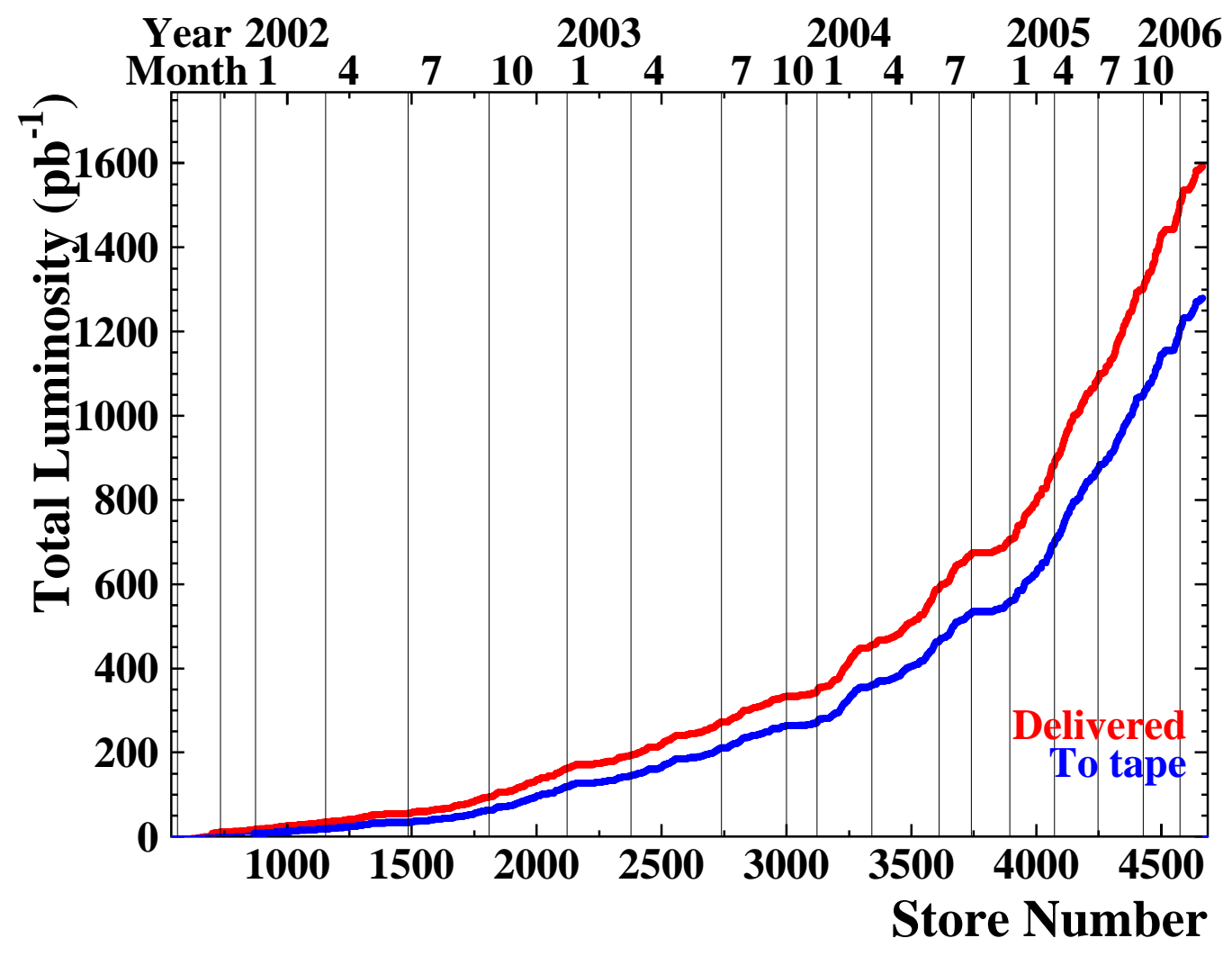

Figure 3.2: Integrated luminosity collected by the CDF detector for Run 2. The top line corresponds to luminosity which is produced at the Tevatron and the bottom line corresponds to luminosity where the collision events are successfully recorded by CDF and available for physics.

collected over a period of time, the instantaneous luminosity is integrated over time:

$$
\mathcal{L}=\int \frac{d \mathcal{L}}{d t} d t
$$

Figure 3.2 shows the integrated luminosity for CDF Run 2. For this analysis, stores between 1120 and 3745 are used corresponding to data taken between March 2002 and August 2004. 


\subsection{CDF Coordinate System}

In order to specify the particle position within the detector, it is useful to introduce a coordinate system with the origin at the interaction point. In Cartesian coordinates, the positive $z$ axis is defined in the proton direction (from west to east), the positive $x$ axis is defined pointing away from the center of the Tevatron (from south to north), and positive $y$ is defined to be pointing upwards. Because of the detector's cylindrical nature, cylindrical coordinates are also used, with $r$ being the radial distance away from the interaction point and $\phi$ being the azimuthal angle going in the counter-clockwise direction when looking along positive $z$. The polar angle $(\theta)$ is used to define the transverse momentum $\left(p_{T}=p \sin \theta\right)$ with $\theta=0$ along the $z$ axis and $\theta=\pi / 2$ along the $y$ axis. Another useful concept

is that of particle rapidity, defined as $Y \equiv \frac{1}{2} \log \frac{E+p_{z}}{E-p_{z}}$ where $E$ is the particle energy and $p_{z}$ is the momentum along the $\mathrm{z}$ axis. For particles traveling close to or at the speed of light, the rapidity can be approximated by the pseudorapidity, defined as $\eta \equiv-\log [\tan (\theta / 2)]$. Pseudorapidities of $0,1,2,3$, and 4, correspond to $\theta$ s of $90^{\circ}, 40.4^{\circ}, 15.4^{\circ}, 5.7^{\circ}$, and $2.1^{\circ}$, respectively.

\subsection{CDF Detector}

The CDF II detector, shown in Figure 3.3, is roughly cylindrical in shape with forward/backward and azimuthal symmetry centered around the interaction point. Many of the components have been upgraded from the CDF I detector because of an increased proton-antiproton collision rate and a higher center of mass energy. The purpose of the CDF detector is to reconstruct particles resulting from proton-antiproton scattering interactions. Because of the multitude of particles per event and types of particles, this task requires various subdetectors. A basic 
overview of the detector is given here with more detail presented in the following sections. Along the $z$ axis is the beam pipe where protons travel from west to east and antiprotons travel from east to west, crossing at the interaction point every 396 ns. Directly surrounding the interaction point is the silicon microstrip tracking detectors, composed of Layer 00, Silicon Vertex Detector (SVX), and Intermediate Silicon Layers (ISL). Directly outside the silicon is the Central Outer Tracker (COT). Together the silicon detectors and the COT are used to measure the track $\vec{p}$ of charged particles. The tracking system is contained within a large solenoidal magnet that produces a field of 1.4 Tesla. Above and below the magnet are the central calorimeters, and on either side of the COT are the plug calorimeters. The calorimeters are used to measure particle energies and their positions. Each calorimeter is composed of an electromagnetic (EM), shower maximum position detector, and hadronic sections. In the far reaches of the detector are muon drift chambers used to identify muons which travel through the calorimeter with minimal interaction. Shielding is used to absorb particles before they reach the muon subdetectors and to protect the entire detector from radiation damage. In between the beam pipe and the plug calorimeters is the Cerenkov luminosity counter, which is used to measure the instantaneous luminosity at CDF.

\subsubsection{Cerenkov Luminosity Counter}

The Cerenkov Luminosity Counter [33, 34], or CLC, determines how much data has been collected at the CDF detector by measuring the instantaneous luminosity. It is designed to measure luminosities up to $\sim 2 \times 10^{32} \mathrm{~cm}^{-2} \mathrm{~s}^{-1}$ with an accuracy of a few percent. The CLC is composed of individual Cerenkov counters arranged in three concentric layers between the beam pipe and plug calorimeter, covering $3.7<|\eta|<4.7$ as shown in Figure 3.4. The counters which look oddly 


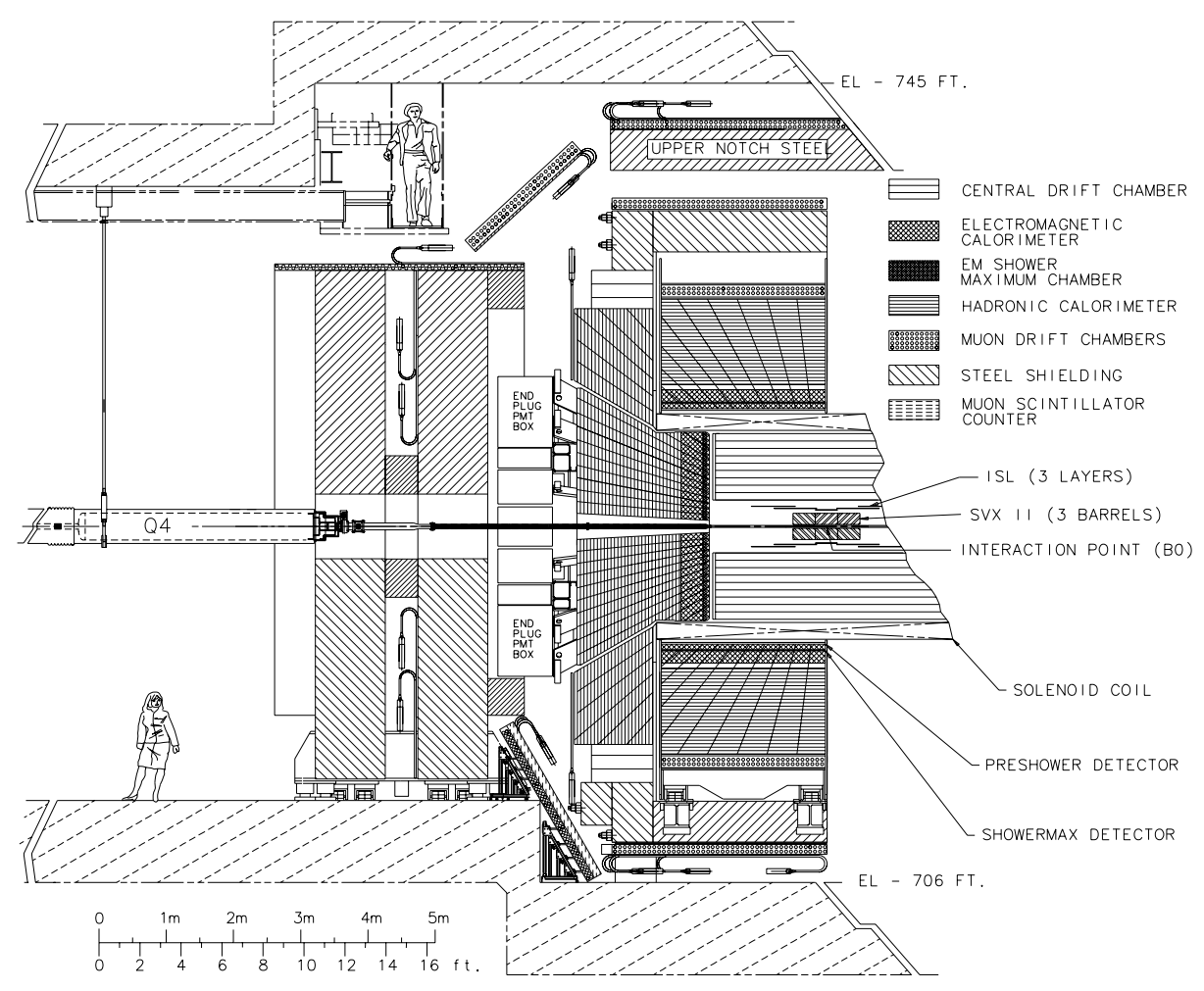

Figure 3.3: Schematic view of the northwest quadrant of the CDF detector. 


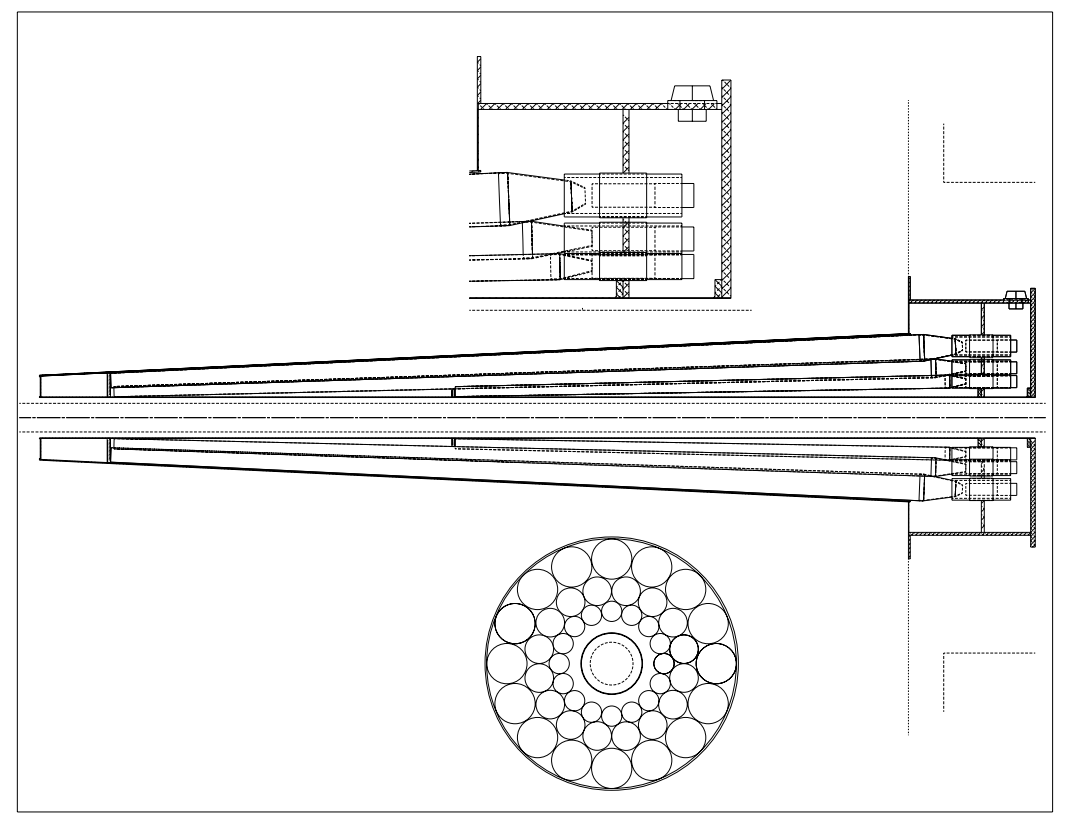

Figure 3.4: Cerenkov Luminosity Counter (CLC) consisting of 3 layers of Cerenkov counters. The middle figure shows a cross section of the Cerenkov counters when looking from the side. The upper figure zooms in on the far end of the counters followed by the photomultiplier tubes. The bottom figure shows a cross section of the CLC when looking from the direction of the beam pipe.

like torpedoes have lengths of $180 \mathrm{~cm}$ for the outer two layers and $110 \mathrm{~cm}$ for the innermost layer. Each layer contains 16 counters.

These Cerenkov counters are filled with isobutane gas that has an index of refraction greater than 1, slowing down the effective speed of light. In the gas, charged particles such as electrons may travel faster than photons creating an effect similar to a sonic boom, except with light instead of sound. As the electron interacts with the gas, it produces light that is focused towards photomultiplier tubes (PMT). The PMTs then convert the light into an electrical signal that can be read out with electronics. In this way the CLC detects fast particles that travel through it. The measured number of particles is related to the average number of interactions per proton-antiproton beam crossing $(\bar{n})$ [34], which can then be 
used to calculate the instantaneous luminosity $\mathcal{L}$ by Equation 3.3, where $f_{B C}$ is the rate of beam crossings at the Tevatron and $\sigma_{p \bar{p}}$ is the total proton-antiproton cross section.

$$
\sigma_{p \bar{p}} \mathcal{L}=\bar{n} f_{B C}
$$

\subsubsection{Tracking Systems}

There are two subdetectors, an inner and outer part shown in Figure 3.5, dedicated to measuring the paths of charged particles, commonly known as tracks. These tracking systems are within a 1.4 Tesla magnet with the field lines traveling toward $+z$. The magnetic field bends positive particles clockwise and negative particles counter-clockwise (looking in the direction of the field). For each track helix there are five important parameters to be measured [35], where the minimum approach refers to the closest point of the track with respect to the primary vertex:

$$
\begin{array}{ll}
\cot \theta & \text { Cotangent of the polar angle at minimum approach. } \\
\text { curvature } & \text { Track curvature, proportional to } 1 / p_{T} . \\
z_{0} & z \text { coordinate at minimum approach. } \\
d_{0} & \text { Impact parameter }- \text { the distance in the } x-y \text { plane from } \\
& \text { the beam position at minimum approach. } \\
\phi_{0} & \text { Azimuthal angle at minimum approach. }
\end{array}
$$

The innermost section of the tracking system is composed of doped silicon microstrips arranged in cylindrical double sided layers with radii from 1.35 to 28 $\mathrm{cm}$ centered around the interaction point. A reverse bias current is placed on each microstrip by applying an electric field within the silicon. When a charged 


\section{CDF Tracking Volume}

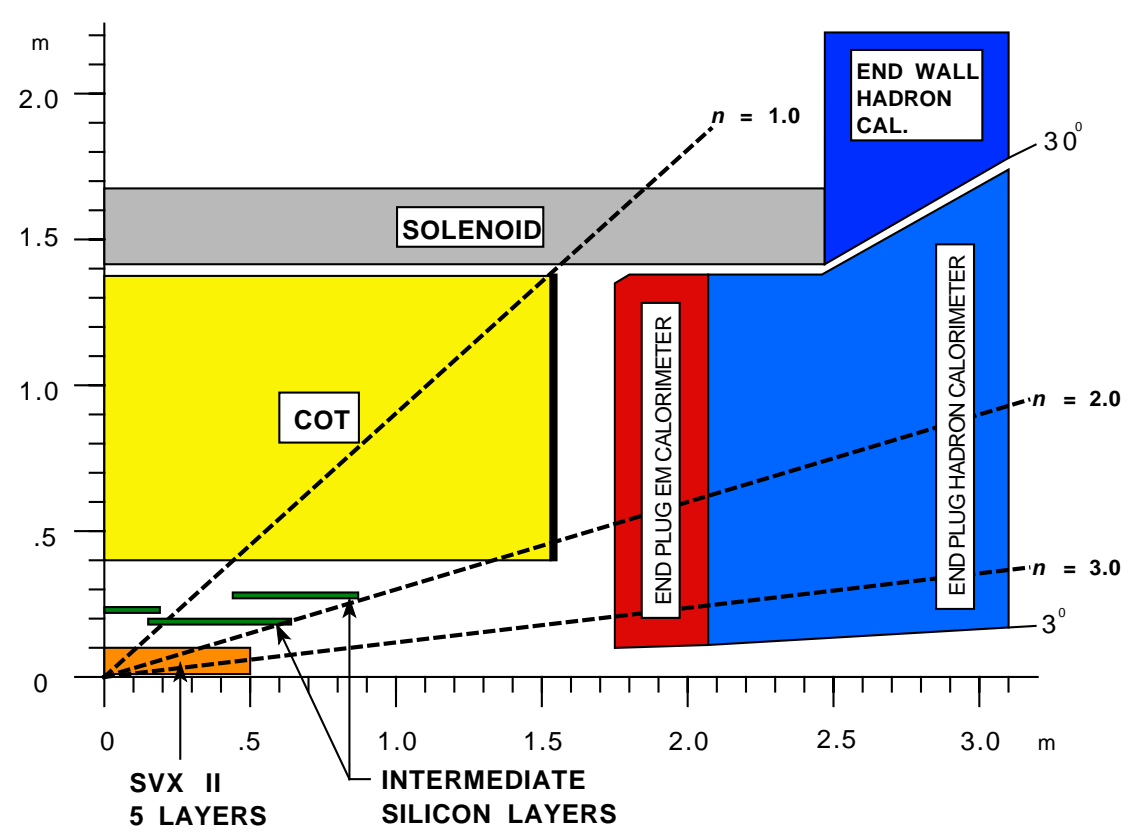

Figure 3.5: Schematic of one quadrant of the CDF tracking system. The inner part consists of a silicon microstrip detector including Layer 00 (not shown), Silicon Vertex Detector (SVX), and Intermediate Silicon Layers (ISL). The outer part consists of the Central Outer Tracker (COT).

particle goes through the silicon it ionizes the atoms, producing electrons and holes - the remaining silicon atom missing one electron and having a net positive charge. In the electric field the electrons travel to one side and the holes in the other leaving an electric signal that can be recorded. In this way the microstrips can measure the time at which a particle goes through it. Due to the narrow width of the strips, this detector has a much finer resolution than other methods of tracking, making it capable of measuring many tracks within a small space.

The silicon tracking system [36], shown in Figure 3.6, consists of three main components: Layer 00 (L00), the five layer Silicon Vertex detector (SVX), and the three layer Intermediate Silicon Layers (ISL). Since the layers are double sided, 
they can measure tracks in the $x-y$ and $r-\phi$ planes. The SVX strip pitch is $\sim 60 \mu \mathrm{m}$ in $\phi$ and $60-141 \mu \mathrm{m}$ in $z$ depending on layer. Together the silicon detectors can measure the $d_{0}$ with a precision of 40 microns and $z_{0}$ to 70 microns.

The outermost section of the tracking system is a drift chamber called the Central Outer Tracker (COT) [37]. The COT is a much larger cylindrical tracking system that uses an argon-ethane gas mixture instead of silicon to measure the paths of charged particles. Potential wires, shaper wires, sense wires, and field panels are placed at various electrostatic potentials in order to create a $1.9 \mathrm{kV} / \mathrm{cm}$ electric field, with the field lines pointing away from the sense wires. As charged particles travel through the COT, the atoms within the gas are ionized and the free electrons drift toward the sense wires in the electric field. Since the electron drift velocity is known, the position of the track can be accurately measured by simply recording the time of the resulting current on the sense wires. It is important that the maximum drift time, which is $177 \mathrm{~ns}$, be smaller than the 396 ns between beam crossings.

The active region of the COT covers $310 \mathrm{~cm}$ along the beam axis and radii between 43 and $132 \mathrm{~cm}$, located just outside the ISL. It is separated into 8 superlayers with the sense wires in the odd layers tilted by $2^{\circ}$ to measure the track in $\eta$ as well as in the $r-\phi$ plane. In total there are over 30,000 sense wires divided into 96 layers (12 per superlayer). Positions of the individual wires within a cell of superlayer 2 are shown in Figure 3.7. The COT can measure tracks with a resolution of $\delta p_{T} / p_{T} \sim 0.15 \%$, where $p_{T}$ is inversely proportional to the track curvature. 


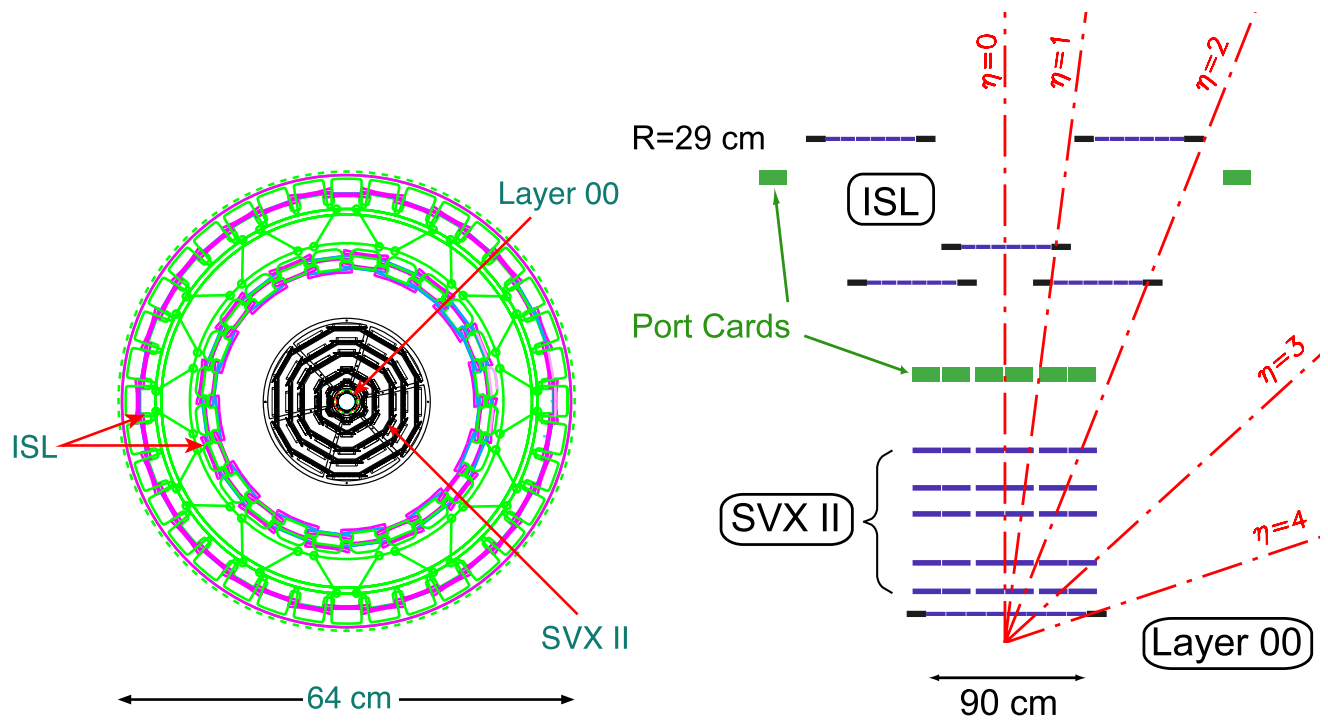

Figure 3.6: Silicon tracking system. On the left is a cross section of the silicon detectors when looking in the direction of the beam pipe. On the right is an $r-z$ view of the silicon detectors, not drawn to scale.

\subsubsection{Calorimeters}

Calorimeters are detectors that measure the energy of incoming particles. They work by slowing down the particles, effectively reducing their energy, by a process called showering. The energy lost is converted into light that is propagated with wavelength shifting fibers, converted into an electronic signal via PMTs, and then recorded with custom electronics. There are two types of showers, electromagnetic (EM) and hadronic, depending on the type of particle. EM showers occur when an electron or photon come into contact with the calorimeter causing the electrons to radiate photons (bremsstrahlung) and photons to convert into electron-positron pairs (pair production or photon conversions). When enough of the energy is deposited, shower maximum is reached, and the electrons will predominately lose their remaining energy through ionization, while photons mainly lose energy through Compton scattering. For EM showers there are two quan- 


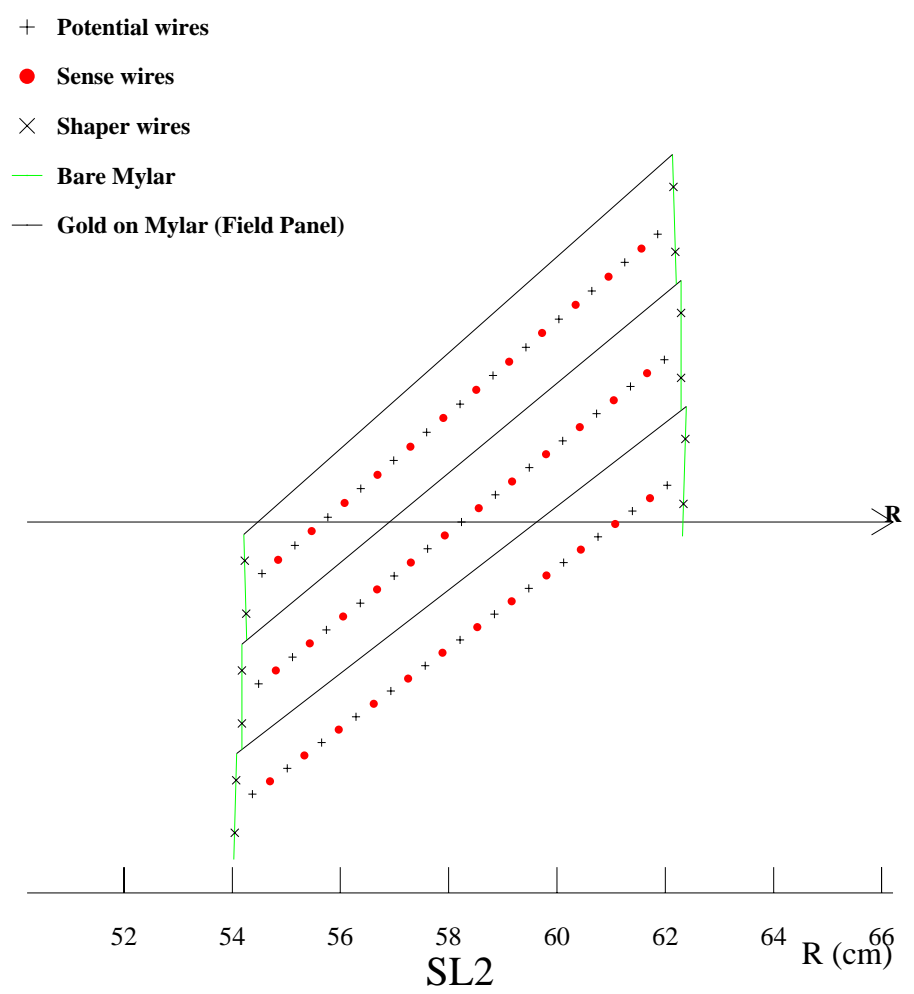

Figure 3.7: Schematic of a single COT cell within superlayer 2.

tities of particular interest: the radiation length $\left(X_{0}\right)$, which is the distance an electron travels before losing 1/e (roughly one third) of its original energy; and the Moliere radius, the transverse radius of the shower at shower maximum. Both quantities are proportional to the atomic number $(\mathrm{Z})$ of the material. Hadrons typically interact with the material's nuclei via the strong interaction and therefore their showering properties will be different. An important distinction is that hadronic showers penetrate deeper into the material than EM showers.

At CDF there are two main calorimeters: the central calorimeter covering $|\eta|<1.1$, and the plug calorimeter covering $1.1<|\eta|<3.6$. They are separated into three components: the EM calorimeter, the shower maximum detector 
located $\sim 6 X_{0}$ within the EM calorimeter and dedicated to making precise position measurements, and the hadronic calorimeter located directly after the EM section. The calorimeters consist of alternating layers of scintillators and a heavy material either lead (central EM, plug EM), steel (central hadronic), or iron (plug hadronic). A scintillator is a transparent material that produces visible light when high energy particles travel through it. These are sampling calorimeters since the energy measured is only a fraction of the total energy. In order to determine the total shower energy, the amount of measured energy in the calorimeter is scaled up by a value either calculated from test beam measurements, from precise particle measurements such as reconstructing the mass of the $\mathrm{Z}$ boson, or from other calibrations.

The EM and hadronic calorimeters have azimuthal symmetry and are segmented into $15^{\circ} \phi$ wedges, except for the wedges in the plug calorimeter between $1.10<|\eta|<2.11$ that cover $7.5^{\circ}$ each. The calorimeters are also segmented into $\eta$ towers, with towers $0-9$ in the central region and 10-21 in the plug region (Fig. 3.8). The central EM calorimeter (CEM) [38] has 20-31 sampling layers depending on the tower. The CEM covers $18 X_{0}$ and has an energy resolution of $13.5 \% / \sqrt{E} \oplus 1 \%$, where $E$ is given in $\mathrm{GeV}$. The first layer of the CEM is called the central preshower $(\mathrm{CPR})$ and is read out separately. The central hadronic calorimeter (CHA) [39] is positioned directly behind the CEM and consists of 48 sampling layers with a resolution of $\sigma(E) / E=0.5 / \sqrt{E}(\mathrm{GeV})$. In between the CHA and the plug calorimeter is the wall hadronic calorimeter (WHA), built with alternating layers of scintillator and steel. The plug EM calorimeter (PEM) [40] contains 23 sampling layers including the preshower layer (PPR) and is 21 $X_{0}$ long. The energy resolution of the PEM is $14.4 \% / \sqrt{E} \oplus 0.7 \%(\mathrm{GeV})$. The plug hadronic calorimeter (PHA) [41] is also contains of 23 scintillator layers and has a resolution of $\sim 80 \% / \sqrt{E} \oplus 5 \%(\mathrm{GeV})$. 


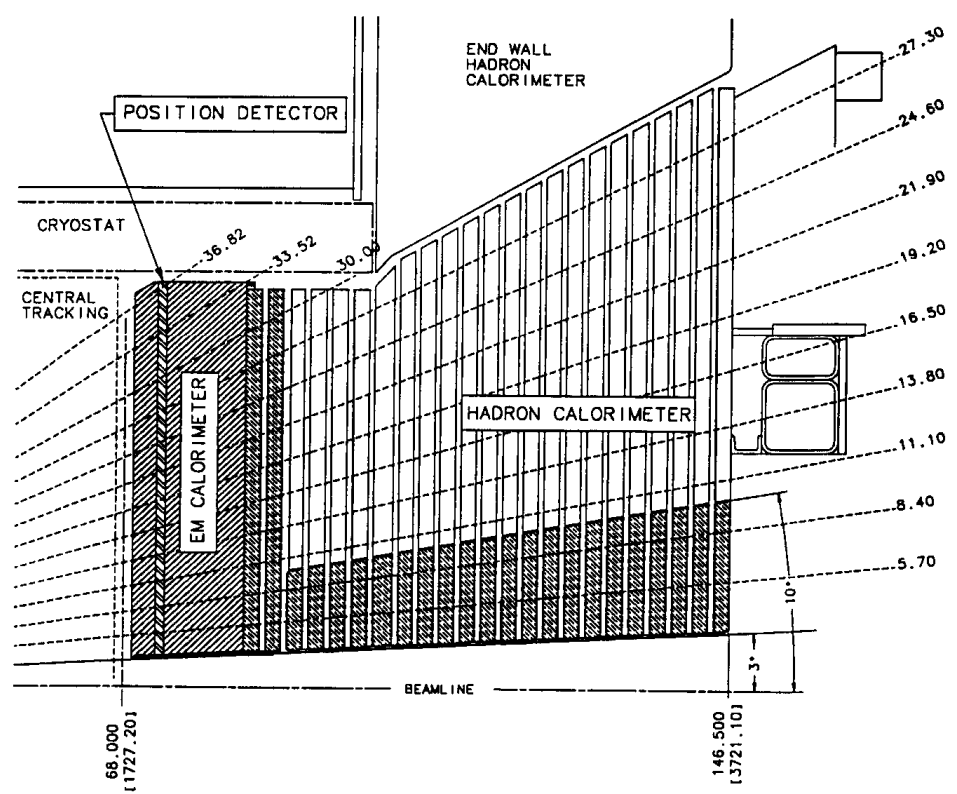

Figure 3.8: Schematic of one quadrant of the plug calorimeter including the EM calorimeter, hadronic calorimeter, and shower maximum detector, labeled "position detector," embedded in the EM section. The plug calorimeter covers $1.1<|\eta|<3.6$ and has the full $2 \pi \phi$ coverage.

The electromagnetic shower maximum detectors are designed specifically to accurately measure the shower position and transverse energy profile in two dimensions. The central shower maximum detector (CES) measures charge deposition along 128 strips and 64 wires running perpendicular to each other in each of the $24 \phi$ wedges. The strips measures the position along the $x$ axis and the wires along the $z$ axis with a resolution of $2 \mathrm{~mm}$ for electrons and photons with $E$ $=50 \mathrm{GeV}$. The plug shower maximum detector (PES) [42] is composed of eight $45^{\circ}$ wedges per plug with two layers of $5 \mathrm{~mm}$ wide scintillating strips, as shown in Fig. 3.9. In total there are 6400 strips, measuring the shower position with a precision of approximately $2 \mathrm{~mm}$. 


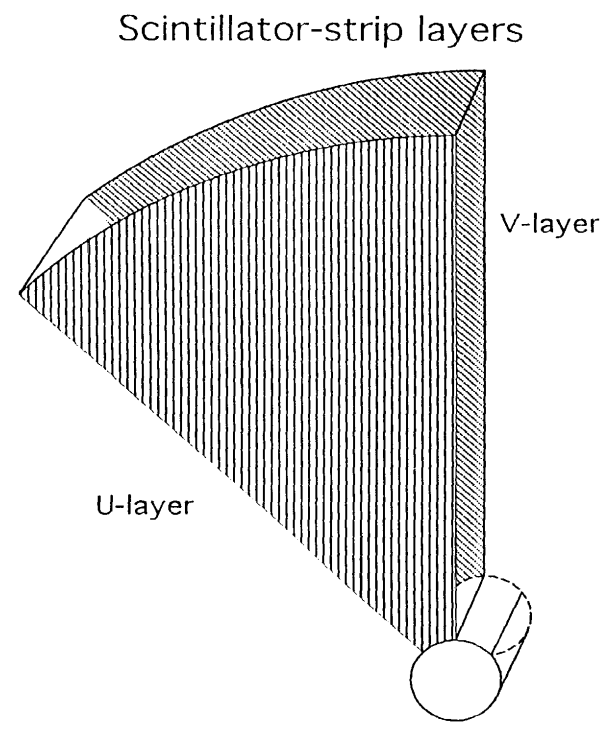

Figure 3.9: Strip layout within one wedge of the plug shower maximum (PES) detector.

\subsubsection{Muon Detectors}

Located behind the hadronic calorimeter are four separate muon detectors: CMU, CMP, CMX, and IMU [43]. The material between these detectors and the interaction point is meant to absorb all other electromagnetic particles besides muons, since muons are only minimally interacting and travel straight through heavy materials. The amount of material is measured in pion interaction lengths $(\lambda)$, the distance for which a pion has a 1/e probability of not interacting with nuclei.

The muon detectors are composed of drift chambers and scintillation counters covering an $|\eta|$ range out to 1.5, as shown in Figure 3.10. The CMU detector includes a four layer drift chamber directly behind the hadronic calorimeter with $\sim 5.5 \lambda$ in front of it. The layers are divided into rectangular drift cells each with a single sense wire to detect charged particles. The detector covers $|\eta|<$ 0.6 and can resolve muons with a minimum $\mathrm{p}_{T}$ of $1.4 \mathrm{GeV} / \mathrm{c}$. Another set of 


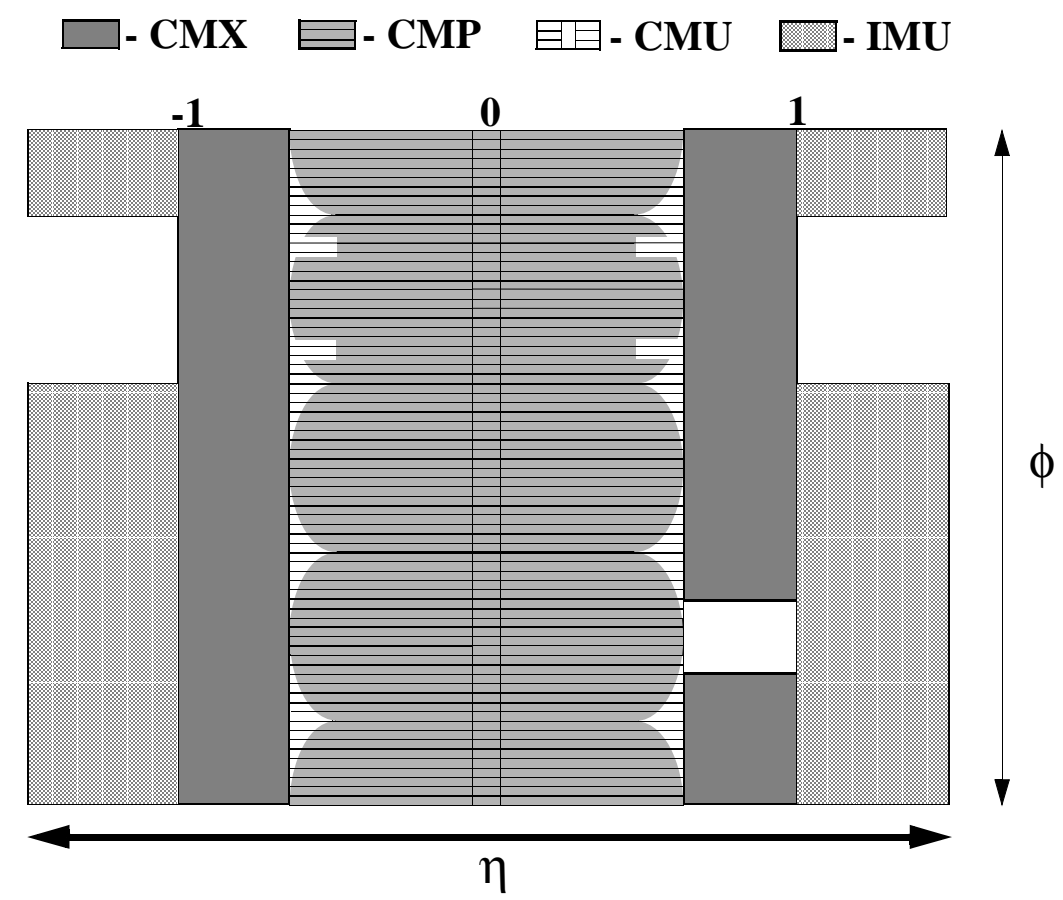

Figure 3.10: Location of the muon detectors in the $\eta-\phi$ plane.

muon drift chambers (CMP) sit behind an additional $60 \mathrm{~cm}$ of steel, adding 2.3 $\lambda$. Similar to the CMU, the CMP is also composed of individual drift cells in four layers and covers $|\eta|<0.6$. On the outside of the CMP drift chambers is a single layer of scintillation counters. All together, the CMP can detect muons down to $\mathrm{p}_{T}$ of $2.2 \mathrm{GeV} / \mathrm{c}$. The CMX detector, composed of conical sections of drift chambers and scintillation counters, extends the muon $|\eta|$ coverage from 0.6 to 1.0. This subdetector sits behind a minimum of $6.2 \lambda$ and can measure muons with a minimum $\mathrm{p}_{T}$ of $1.4 \mathrm{GeV} / \mathrm{c}$. Finally the IMU, which is completely new for CDF Run II, is also composed of drift cells and scintillator counters behind 6.2-20 $\lambda$ extending the $|\eta|$ coverage out to 1.5. However this subdetector is not used since the phoenix tracking algorithm employed for forward tracking does not recognize muon tracks, which are required for muon identification. 


\subsection{Event Triggers and Data Acquisition}

At the Tevatron, collisions occur at a rate of roughly $2.5 \mathrm{MHz}$. Since data can only be written to tape at a rate of $\sim 50 \mathrm{~Hz}$, there needs to be a system that decides which events to store. In order to select these events, a three level CDF trigger system [54] is employed, as shown in Figure 3.11. Each level uses more restrictive criteria, reducing the event rate such that it can be handled by the next level. Level 1 uses custom designed hardware to find tracks using the extremely fast tracker (XFT), muon stubs, calorimeter towers above a certain energy threshold, and missing transverse energy. The XFT is a device for finding tracks with a minimum $p_{T}$ of $1.5 \mathrm{GeV} / \mathrm{c}$ in the four axial layers of the COT. Events are kept in a 42 clock cycle storage pipeline while awaiting the level 1 trigger decision, with each clock cycle lasting 132 ns. The level 1 trigger decision takes approximately $5 \mu$ s and accepts slightly less than 20,000 events per second. If the event is accepted, it is entered into a 4 event buffer awaiting the level 2 trigger decision. If an event is accepted by level 1 while all four level 2 buffers are full, then the event is lost. If no events are lost, this is known as "deadtimeless". The level 2 trigger is also composed of custom hardware and can perform more complex event reconstruction such as calorimeter clustering and higher quality track reconstruction. The level 2 trigger decision lasts about $20 \mu$ s and accepts 150-300 events per second. If the event is accepted by the level 2 trigger, the raw data from the front-end electronics associated with each subdetector is sent to VME crates which contain several buffers (VRBs). Once all the data is collected for a certain event, computers (SCPUs) send the data to the level 3 computer farm. Assembling all the raw data from a single event is known as event building and is shown in Fig. 3.12. The level 3 trigger fully reconstructs the event in order to determine whether to accept it. Only 50 events per second pass the final 
trigger decision. Events satisfying the level 3 requirements are stored in datasets on tapes, classified according to what trigger(s) it passed.

\subsubsection{The Shift Crew}

Under normal detector operation, four people are required to run the experiment at a time and are responsible for collecting data as efficiently as possible. Collectively, these four individuals are known as the shift crew. This shift crew consists of one person called the "DAQ ACE" who is at the helm of the experiment taking the data runs and managing the DAQ resources, another person called the "monitoring ACE" who ensures that each subdetector is running under the correction conditions, a person called the "consumer operator" who monitors the data quality of each subdetector, and a "scientific coordinator" who directs shift crew activities such that they coincide with the goals and day to day needs of the CDF collaboration. When problems arise, it is the job of the shift crew to notify the appropriate experts so that it is resolved as soon as possible. Since direct contact with the detector is off limits during Tevatron operation, sufficient time between stores is allocated for experts to access the detector and apply the appropriate solutions if needed. If data is taken while one of the subdetectors is operating in a compromised state, the shift crew keeps record of which subdetectors are involved and for what data runs. The shift crew is also responsible for conducting detector calibrations.

\subsubsection{Good Run Requirements}

Even if data passes the trigger requirements, it might not be suitable for an analysis. For this reason, good run requirements are established to determine which data runs should be used and which ones should not. As an example, this 


\section{Dataflow of CDF "Deadtimeless" Trigger and DAQ}

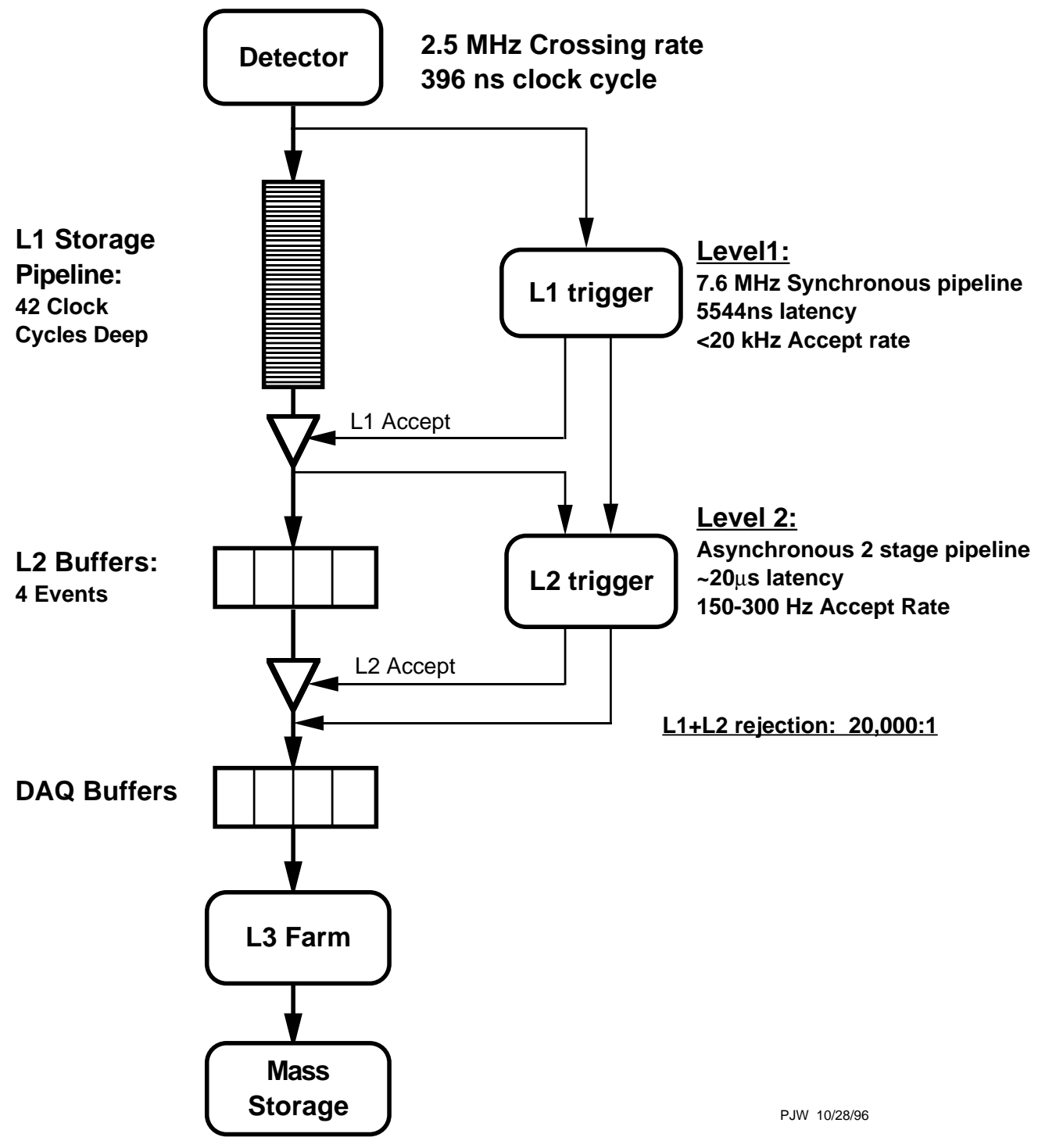

Figure 3.11: Block diagram depicting the CDF trigger system and path of online data culminating in storage on tapes. 


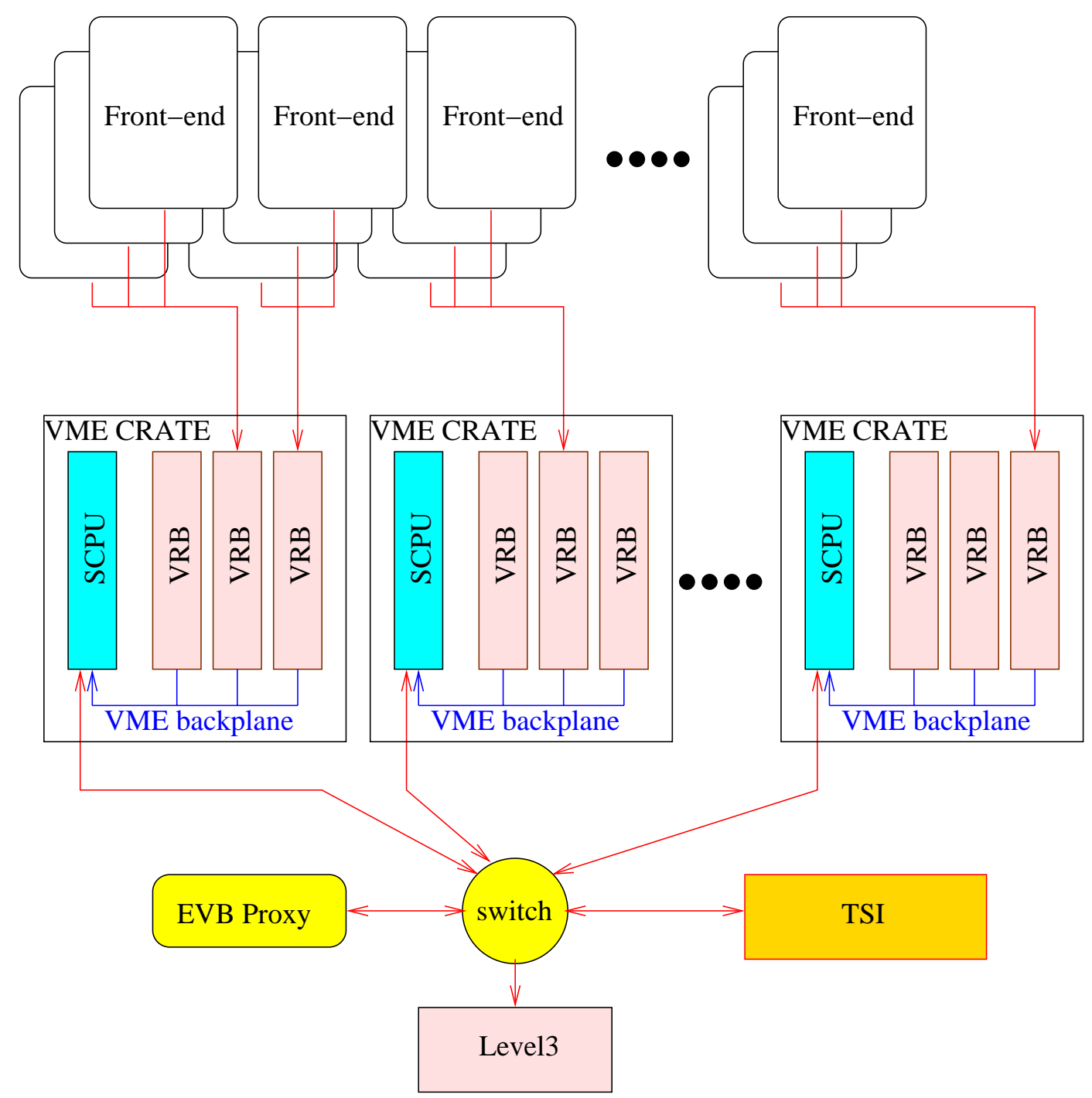

Figure 3.12: Block diagram of the CDF event builder in which the raw data from the front-end electronics is collected and sent to the level 3 trigger. 
thesis searches for events with electrons and muons and therefore requires runs where the tracking detectors, the calorimeters, the muon detectors, the CLC, and their readout are all working properly. Since the datasets include all runs which pass the trigger, it is up to the experimenter to exclude runs which fail the good run criteria.

\subsubsection{CDF Data Reconstruction Software}

The data from level 3 corresponds to single channels: energy within a single calorimeter tower, individual COT wire hits, etc. This information is stored in "D banks" corresponding to the appropriate subdetector. For example, all CEM towers with energy above a certain threshold are stored in CEMD banks, and COT hits are stored in COTD banks. One component of the CDF software, written mainly in $\mathrm{C}++$, uses the information from the $\mathrm{D}$ banks to reconstruct more useful quantities, such as tracks, muon stubs, calorimeter clusters, and missing energy. Basic reconstruction is described below.

There are several methods of finding tracks. Typically, tracking algorithms start with $\mathrm{r}-\phi$ hits within the COT using a five dimensional fit to find a track helix. Once a helix is found, silicon hits and other hits can be attached if they are within a given proximity. These tracks are known as "outside-in." Another type of tracking algorithm, used outside of the central region $(|\eta|<1)$, is called phoenix tracking. Instead of looking for tracks starting from hit positions, phoenix tracking start with a primary vertex and an electromagnetic shower maximum cluster. Using the energy of the cluster, the curvature of two possible seed tracks are determined, for the two possible electric charges. If there are enough hits along one of the possible seeds, a phoenix track is formed. Tracks are stored in collections, for example there are the CdfTrackColl and the PheonixTrackColl, that contain 
all tracks of the appropriate type for a given event.

Tracks are also used to form primary vertices if enough of them all point back to the same location. The vertex quality depends on how many tracks are used to form the vertex and the type of tracks. The primary vertices are used as a measure of the position of a proton-antiproton collision.

Muon stubs are created from three or four correlated hits on the layers of an individual muon detector. Information related to the stubs are stored in a collection called MuonStubColl. For muon stubs that roughly align with the $\vec{p}$ of a track, the information is combined to form a muon candidate collections called CdfMuonColl.

In the electromagnetic calorimeter, clusters are formed by identifying seed towers containing energy above a given threshold and attaching surrounding towers that also contain sufficient energy. For the shower maximum detectors, clusters are also formed using seed methods, except this is done for each of the two layers separately. The information related to calorimeter clusters are stored in collections for each subdetector: CdfEmClusterColl, CesClusterColl, CprClusterColl, and PlugStripColl. If a track roughly matches in position with a calorimeter cluster, the information is combined to form an electron candidate collections called CdfEmObjectColl.

Neutrinos almost always travel right through the detector without any direct observation. Nonetheless, they can be inferred through a quantity called missing transverse energy $\left(\mathbb{E}_{T}\right)$. $\mathbb{E}_{T}$ is defined by:

$$
E_{T}=\left|\sum_{i} E_{T}^{i} \hat{n}_{i}\right|
$$

where $\mathrm{E}_{T}^{i}$ is the transverse energy contained within calorimeter tower $i$ and $\hat{n}_{i}$ is the transverse component of the unit vector pointing from the interaction point 
to the same tower. $\not_{T}$ is corrected for the $p_{T}$ of any muons in the event since muons do not deposit all their energy in the calorimeter. Since the particle beams travel along the $z$ axis, $\mathbb{E}_{T}$ is expected to be small unless a neutrino or any other particle escapes detection. When measuring $\mathbb{E}_{T}$, values can slightly differ from 0 , in the range of $\lesssim 5-10 \mathrm{GeV}$ due to particle showers where some of the energy goes into gaps in the calorimeter or due to undetected particles that travel near the beam line.

All of the banks and collections are stored for each proton-antiproton collision in the event record. The software used to create the event record is the same for every analysis performed at CDF. In addition, there are software tools that organize the information from the collections, into a more useful format using object-oriented programming. For example, organizing all the useful electron related quantities into a single electron collection facilitates analyses that look for electrons in the final state.

The last piece of analysis software is written by the experimenter to perform the specific tasks which are required for the individual analysis.

\subsubsection{Event Simulation}

Computer simulated proton-antiproton events are important in testing our understanding of what happens in actual events, for studying a particular type of interaction, and making physics predictions. Monte Carlo (MC) event generators are used to create simulated events that are as similar as possible to actual events. Therefore these generators must include a good understanding of particle

physics for a wide range of particles over a wide range of energies. Despite the challenge of developing such a complicated piece of software, several generators exist, including PYTHIA [44] and ISAJET [30]. 
Additional simulation software is needed to describe how the particles interact with the detector. This requires an accurate description of the detector geometry and material content, provided in the CDF software. GEANT [45] is used to describe how the particles interact with the detector. Since a full simulation of an electromagnetic shower would be overly time and resource consuming, the quicker GFLASH package [46] is used to parameterize the showers. Once the event is simulated, the same banks and collections found in actual events are created as well as banks storing the generated particle information. 


\section{CHAPTER 4}

\section{Particle Identification}

During each collision, many particles traverse the CDF detector simultaneously. Depending on a particle's mass, charge, and lifetime, it may be detected by one or more of the subdetectors. To identify particles, the output from multiple subdetectors is combined in a similar fashion to the way in which we combine the information from our five senses to help recognize objects encountered in day to day life. Particle identification is not simple since several particles may exhibit similar behavior. In addition, not all particles are useful for this analysis. We are primarily interested in charged leptons consistent with the decay of a heavy suspersymmetric particle or other massive object that has not been discovered yet.

To get a feeling for the challenges involved in particle identification, one may try the following exercise. Imagine being visited by an alien from another planet that has no knowledge of animals on Earth, that asks how it can identify a dog. At first thought, this may seem like an easy task since most dogs can instantly be recognized simply by looking at it, but this recognition comes from years of familiarity and is not easily communicated. One might start out by listing a dog's properties such as its four legs, tail, etc. Unfortunately this description is not only true for dogs, but for cats, horses, buffalo, and many other animals. In order to exclude other animals, more specific characteristics about dogs must be added to the description such as the way a dog barks. Yet the more specific the 
criteria, the more likely it is to exclude certain kinds of dogs that do not meet the description. This is exactly the difficulty in particle identification: listing a set of criteria that is true for a high percentage of one particular particle but very rarely for any other. On some level particle identification is easier since there are less variations with particle interactions than there are types and characteristics of dogs.

This chapter explains how information from several subdetectors is combined to identify particles consistent with the goals of this analysis. Since particle identification is an important component of many analyses conducted at CDF, most of the information presented in this chapter has been developed through a collaborative effort.

\subsection{Electron Identification}

The basic electron requirements include a track in the COT and/or SVX subdetectors combined with an EM shower in either the central or plug calorimeters. If a sample is collected containing all objects that satisfy these two requirements, the sample would consist of electrons, charged pions, charged kaons, and neutral particles $\left(\pi^{0} \mathrm{~s}, \eta \mathrm{s}\right.$, etc. $)$ that overlap with charged tracks, among others. All particles within this sample that are not electrons are considered backgrounds (for a more complete definition of backgrounds, see Sec. 5.3.) To increase the electron purity of the sample, other identification (ID) cuts are imposed, described in Sec. 4.3.

Electrons are classified by subdetector, either central or plug, and by category of ID cuts passed. For central electrons, there are both tight (TCE) and loose (LCE) electron categories as a tool for optimizing the analysis. If the number 
of background events are a significant fraction of the total number of events in the electron sample, then the TCE cuts are used in order to minimize the background contamination, otherwise the LCE cuts are used to maximize the number of electrons in the electron sample. The distinction between tight and loose ID cuts is only made for central electrons. Plug electrons are considered to be loose, based on the lower purity of plug electron samples.

Another distinction is made for plug electrons based on track type: standalone or Phoenix. The phoenix electrons (plug electrons with phoenix tracks) have a higher ID efficiency and are used to identify plug electrons with $E_{T}>$ $15 \mathrm{GeV}$ while stand-alone plug electrons have lower backgrounds and are therefore used to identify plug electrons with $E_{T}<15 \mathrm{GeV}$.

\subsection{Muon Identification}

Since muons are roughly 200 times heavier than electrons, high-momentum muons tend to travel right through the detector, only losing a small amount of their initial energy. The basic muon requirements include a track in the COT, an electromagnetic cluster associated with a minimum ionizing particle, and a stub in one of the muon drift chambers. Muons are classified by the subdetector they pass through: CMUP (CMU and CMP) or CMX. Additionally, CMIO muons are accepted without the EM cluster and stub requirements if the track is projected to pass through an area not covered by the muon chambers, most often around $\eta=0$. Since a CMIO muon is essentially just a track, electrons and jets that travel through gaps within the calorimeter may be misidentified as CMIO muons. Therefore, CMIO muons that travel through the calorimeter, which are most likely to be muons, are distinguished in the event selection (Sec. 6.1) from CMIO muons that travel through the gaps and may include electrons, jets, and muons. 
Similar to electrons, identification cuts are used to increase the purity of the muon samples (see Sec. 4.3). Since there is no stub requirement for CMIO muons, there is a much higher chance for a jet to be mistaken for this type of muon and therefore some of the other cuts are more stringent than for CMUP and CMX muons.

\subsection{Lepton Identification Cuts}

The selection cuts which are used in this analysis to increase the purity of both electron and muon samples are described below:

$E_{T}$ : Transverse energy of the electron measured in the EM calorimeter. Backgrounds decrease as $\mathrm{E}_{T}$ increases.

$p_{T}$ : Transverse momentum of the most energetic track matched to the electron or muon. Typically there is only one associated track.

$Z_{0}$ : Position of the primary vertex on the $z$ axis of the detector. There is greater detector coverage if the electron originates closer to the center.

\# COT Segments: A track quality cut requiring a minimum number of axial and stereo layers with at least 5 hits in each layer. Energetic electrons and muons are expected to have excellent track quality.

Isolation: Maximum value of $E_{T}^{\text {iso }} / \mathrm{E}_{T}\left(E_{T}^{\text {iso }} / \mathrm{p}_{T}\right)$ allowed for electrons (muons) in units with $c=1$. $\mathrm{E}_{T}^{i s o}$ is the transverse energy within a cone of radius $\Delta R=$ $\sqrt{\Delta \eta^{2}+\Delta \phi^{2}}<0.4$ not due to the lepton. This cut reduces the number of jets, 
since they tend to be much less collimated than electrons and muon.

Track $d_{0}$ : Distance in the $x-y$ plane between the track and the primary vertex as measured by either the SVX or COT detectors. Since the resolution of the silicon detectors is better than the that of the COT, a tighter $d_{0}$ cut is used for tracks with SVX hits than for tracks detected exclusively with the COT.

Fiduciality: This term refers to areas of a subdetector in which there is optimal performance. Performance can be diminished, for example, at an edge of a subdetector. Electrons, CMUP muons, and CMX muons are required to be fiducial. CMIO muons are required not to be fiducial to the CMU and CMP, or CMX subdetectors

\subsubsection{Electron Identification Cuts}

The selection criteria used for identification of tight and loose central electrons are listed in Table 4.1, while the identification requirements for plug electrons are listed in Table 4.2. The electron identification cuts are described below:

Had/Em: Ratio of the total energy in the hadronic calorimeter divided by the energy in the EM calorimeter. This cut helps to differentiate electrons and photons from jets, since jet showers tend to penetrate deeper into the calorimeter.

E/p: Transverse energy of the electron divided by the transverse momentum

of the highest $p_{T}$ track. Since $E^{2}-p^{2} c^{2}=m^{2} c^{4}$, this ratio is close to one (in units with $c=1$ ) for particles where their momentum is much greater than their mass, such as electrons. For jets, $E$ corresponds to the energy of all the component hadrons, while $p$ is measured for a single charged particle within the jet, 
and therefore $E / p$ is often much larger than one.

$\mathrm{L}_{s h r}$ : This variable compares the energy deposition between towers of an observed CEM cluster to that of a "true" electron or photon shower as determined from test beam measurements [55]. Test beam measurements involve bombarding the subdetector with electrons or other particles of known energy before the subdetector is inserted in its final resting place at $C D F$. $\mathrm{L}_{s h r}$ is defined as:

$$
L_{s h r}=0.14 \frac{\sum_{i}\left(E_{i}-P_{i}\right)}{\sqrt{\left.0.14^{2} E_{E M}+\Sigma_{i}\left(\Delta P_{i}\right)^{2}\right)}}
$$

where the sum is over the towers adjacent to the seed tower in the same calorimeter wedge. $E_{i}$ is the measured energy contained in the tower while $P_{i}$ is the predicted energy for similar energy configurations determined by previous tests. $E_{E M}$ is the total energy in the electron EM cluster.

CES $\mathrm{Q} * \Delta X, \Delta Z$ : Differences in the position of the extrapolated track with the energy centroid measured by the strips $(\Delta X)$ or wires $(\Delta Z)$ of the CES. Q is the sign of the electric charge $( \pm 1)$ determined by the track curvature in the magnetic field.

CES $\chi^{2}$ : "Goodness of fit" between the lateral shower shape of the energy in the CES subdetector and the electron shower shape measured during test beam measurements. There are two $\chi^{2}$ variables, one for the strips and one for the wires.

PEM $\chi^{2}$ : A comparison between the observed energy in the $3 \times 3$ PEM towers containing a shower to the expected energy shower shape from test beam measurements. This variable is the plug equivalent of the central $\mathrm{L}_{s h r}$ cut. 


\begin{tabular}{|c|c|c|}
\hline \multicolumn{3}{|c|}{ Central Electron Identification Requirements } \\
\hline ID Cut & Tight (TCE) & Loose (LCE) \\
\hline $\mathrm{E}_{T}$ & \multicolumn{2}{|l|}{$\geq 5 \mathrm{GeV}$} \\
\hline $\mathrm{p}_{T}$ & \multicolumn{2}{|c|}{$\geq 4 \mathrm{GeV} / \mathrm{c}$} \\
\hline Track $\left|Z_{0}\right|$ & \multicolumn{2}{|l|}{$<60 \mathrm{~cm}$} \\
\hline Track $\left|d_{0}\right|$ & \multirow{2}{*}{\multicolumn{2}{|c|}{$<0.2 \mathrm{~cm}$ for \#SVX Hits $=0$}} \\
\hline & & \\
\hline COT Axial Segments & \multicolumn{2}{|c|}{$\geq 3$ (with 5 hits each) } \\
\hline COT Stereo Segments & \multicolumn{2}{|c|}{$\geq 2($ with 5 hits each $)$} \\
\hline $\mathrm{Had} / \mathrm{Em}$ & \multicolumn{2}{|c|}{$<0.055+0.00045 * \mathrm{E}$} \\
\hline$E_{T}^{i s o} / \mathrm{p}_{T}$ & \multicolumn{2}{|l|}{$<0.1$} \\
\hline $\mathrm{E} / \mathrm{p}$ & $<2$ or $\mathrm{E}_{T}>100 \mathrm{GeV}$ & - \\
\hline $\mathrm{L}_{s h r}$ & $<0.2$ & - \\
\hline $\mathrm{Q} * \Delta X$ & $-3<\mathrm{Q} * \Delta X<1.5 \mathrm{~cm}$ & - \\
\hline$|\Delta Z|$ & $<3 \mathrm{~cm}$ & - \\
\hline$\chi_{\text {strip }}^{2}$ & $<10$ & - \\
\hline
\end{tabular}

Table 4.1: Lepton identification requirements for central electrons.

PES 5/9: A PES cluster has 9 strips centered around the strip with the highest energy. This variable determines how localized the energy shower is by measuring the ratio of energies of the middle 5 strips divided by all 9 strips. Since electrons are expected to have narrower showers in the transverse direction than for jets, the PES $5 / 9$ variable is expected to have smaller values for electrons than for jets. 


\begin{tabular}{|l|c|c|}
\hline \multicolumn{2}{|l|}{ Plug Electron Identification Requirements } \\
\hline ID Cut & Phoenix & Stand-Alone \\
\hline $\mathrm{E}_{T}$ & $>15 \mathrm{GeV}$ & $5-15 \mathrm{GeV}$ \\
\cline { 2 - 3 }$|\eta|$ & $1.2-2.0$ \\
Track $\left|Z_{0}\right|$ & $<60 \mathrm{~cm}$ \\
Had/Em & $<0.05$ \\
PES $5 / 9$ & 0.65 for both U \& V layers \\
$\chi_{P E M}^{2}$ & $<10$ \\
$E_{T}^{i s o} / \mathrm{p}_{T}$ & $<0.1$ \\
$\mathrm{E} / \mathrm{p}$ & - & $<3$ \\
\cline { 2 - 3 }
\end{tabular}

Table 4.2: Lepton identification requirements for plug electrons.

\subsubsection{Muon Identification Cuts}

The selection criteria used for identification of stubbed muons are listed in Table 4.3, while the identification requirements for stubless muons are listed in Table 4.4. The muon identification variables are described below:

Em Energy, Had Energy, Total Energy: Since high- $p_{T}$ muons are minimum ionizing particles, they are expected to deposit less energy in the calorimeter than electrons or jets, where the energy deposition is a function of the muon $p_{T}$. Therefore, there are maximum requirements on the amount of energy in the EM and Hadronic calorimeters associated with the muon as a function of $p_{T}$. A total energy cut requires the combined energy in the EM and Hadronic calorimeters associated with the muon to be above a small value.

Stub $\Delta X$ and $\chi^{2}$ Cuts: A set of correlated hits within one of the muon sub- 
detectors is referred to as a stub. To identify muons, the stub is required to be consistent with the corresponding COT track by applying a $\Delta X$ cut. Similar to the CES $\Delta X$, the muon $\Delta X$ measures the difference between the extrapolated COT track and the stub position. For lower momentum muons, interactions with the detector can cause the particle to be deflected away from the initial direction making the $\Delta X$ cut less efficient. Therefore, muons with $p_{T}<20 \mathrm{GeV} / c$ may satisfy either the $\Delta X$ cut or a $\chi^{2}$ cut that uses the distance between the extrapolated COT track and the muon stub, but also takes multiple scattering into account [56].

Exit Radius $(\rho)$ : The radius at which the track crosses the $|z|$ position of the COT end-cap. This cut is applied for CMX muons which fire the single high- $p_{T}$ muon trigger, since many muons with a small $\rho$ value $(<140 \mathrm{~cm})$ fail the CMX trigger. Applying a cut on this variable improves the agreement between the data and the simulation.

\subsection{Lepton Identification Efficiencies}

While the use of lepton ID cuts improves the sample purity, the price paid is a decrease in lepton ID efficiency $\left(\epsilon_{I D}\right)$, where $\epsilon_{I D}$ is the percentage of fiducial leptons that are identified in the detector. To measure the $\epsilon_{I D}$, one must have a sample of high purity leptons before making any lepton ID cuts. The $Z \rightarrow$ $\ell^{+} \ell^{-}$sample is most often used for this purpose since it has very little background. The sample is collected from a single lepton high- $p_{T}$ trigger by requiring one well identified electron or muon plus a second opposite sign object, also known as the

probe lepton, where the combined invariant mass is in the range of a $Z$ boson (an invariant mass distribution for $Z \rightarrow \mu^{+} \mu^{-}$events is shown in Fig. 4.1). The 


\begin{tabular}{|c|c|c|}
\hline \multicolumn{3}{|c|}{ Stubbed Muon Identification Requirements } \\
\hline ID Cut & CMUP Muons & CMX Muons \\
\hline $\mathrm{p}_{T}$ & \multicolumn{2}{|c|}{$\geq 5 \mathrm{GeV} / \mathrm{c}$} \\
\hline Track $\left|Z_{0}\right|$ & \multicolumn{2}{|c|}{$<60 \mathrm{~cm}$} \\
\hline \multirow[t]{2}{*}{ EM Energy } & \multicolumn{2}{|c|}{$<2 \mathrm{GeV}$ for $\mathrm{p}_{T}<100 \mathrm{GeV} / \mathrm{c}$} \\
\hline & \multirow{4}{*}{\multicolumn{2}{|c|}{$\begin{array}{c}<2+\left(\mathrm{p}_{T^{-}}-100\right) * 0.0115 \mathrm{GeV} \text { for } \mathrm{p}_{T}>100 \mathrm{GeV} / \mathrm{c} \\
<3.5+\mathrm{p}_{T} / 8 \mathrm{GeV} \text { for } \mathrm{p}_{T}<20 \mathrm{GeV} / \mathrm{c} \\
<6 \mathrm{GeV} \text { for } 20<\mathrm{p}_{T}<100 \mathrm{GeV} / \mathrm{c} \\
<6+\left(\mathrm{p}_{T}-100\right) * 0.028 \mathrm{GeV} \text { for } \mathrm{p}_{T}>100 \mathrm{GeV} / \mathrm{c}\end{array}$}} \\
\hline \multirow[t]{3}{*}{ Had Energy } & & \\
\hline & & \\
\hline & & \\
\hline Total Energy & \multicolumn{2}{|c|}{$<0.1 \mathrm{GeV}$} \\
\hline \multirow[t]{2}{*}{ Track $\left|d_{0}\right|$} & \multirow{2}{*}{\multicolumn{2}{|c|}{$\begin{array}{l}<0.2 \mathrm{~cm}\left(\text { if } \mathrm{p}_{T}<20 \mathrm{GeV} / \mathrm{c} \text { or \#SVX Hits }=0\right) \\
<0.02 \mathrm{~cm}\left(\text { if } \mathrm{p}_{T}>20 \mathrm{GeV} / \mathrm{c} \text { and \#SVX Hits }>0\right)\end{array}$}} \\
\hline & & \\
\hline COT Axial Segments & \multicolumn{2}{|c|}{$\geq 3$ (with 5 hits each) } \\
\hline COT Stereo Segments & \multicolumn{2}{|c|}{$\geq 2($ with 5 hits each $)$} \\
\hline$E_{T}^{i s o} / \mathrm{p}_{T}$ & \multicolumn{2}{|c|}{$<0.1$} \\
\hline \multirow[t]{2}{*}{ CMU Stub Cuts } & $|\Delta X|<3 \mathrm{~cm} \mathrm{or}$ & - \\
\hline & $\chi^{2}<9$ for $\mathrm{p}_{T}<20 \mathrm{GeV} / \mathrm{c}$ & \\
\hline \multirow[t]{2}{*}{ CMP Stub Cuts } & $|\Delta X|<5 \mathrm{~cm} \mathrm{or}$ & - \\
\hline & $\chi^{2}<9$ for $\mathrm{p}_{T}<20 \mathrm{GeV} / \mathrm{c}$ & \\
\hline \multirow[t]{2}{*}{ CMX Stub Cuts } & - & $|\Delta X|<6 \mathrm{~cm} \mathrm{or}$ \\
\hline & - & $\chi^{2}<9$ for $\mathrm{p}_{T}<20 \mathrm{GeV} / \mathrm{c}$ \\
\hline \multirow[t]{2}{*}{ Exit Radius $(\rho)$} & - & $>140 \mathrm{~cm}$ \\
\hline & - & \\
\hline
\end{tabular}

Table 4.3: Lepton identification requirements for stubbed muons. For formulas with mixed units, $\mathrm{E}_{T}$ is measured in units of $\mathrm{GeV}$ and $p_{T}$ is measured in units of $\mathrm{GeV} / c$. 


\begin{tabular}{|l|c|}
\hline \multicolumn{2}{|c|}{ Stubless Muon Identification Requirements } \\
\hline ID Cut & CMIO Muons \\
\hline $\mathrm{p}_{T}$ & $\geq 10 \mathrm{GeV} / \mathrm{c}$ \\
Track $\left|Z_{0}\right|$ & $<60 \mathrm{~cm}$ \\
EM Energy & $<2 \mathrm{GeV}$ \\
Had Energy & $<3.5+\mathrm{p}_{T} / 8 \mathrm{GeV}$ for $\mathrm{p}_{T}<20 \mathrm{GeV} / \mathrm{c}$ \\
& $<6$ for $\mathrm{p}_{T}>20 \mathrm{GeV} / \mathrm{c}$ \\
Total Energy & $>0.1 \mathrm{GeV}$ \\
Track $\left|d_{0}\right|$ & $<0.2 \mathrm{~cm}\left(\right.$ if $\mathrm{p}_{T}<20 \mathrm{GeV} / \mathrm{c}$ or \#SVX Hits $\left.=0\right)$ \\
& $<0.02 \mathrm{~cm}$ (if $\mathrm{p}_{T}>20 \mathrm{GeV} / \mathrm{c}$ and \#SVX Hits $\left.>0\right)$ \\
COT Axial Segments & $\geq 3($ with 5 hits each) \\
COT Stereo Segments & $\geq 3$ (with 5 hits each) \\
$E_{T}^{\text {iso } / \mathrm{p}_{T}}$ & $<0.1$ \\
\hline
\end{tabular}

Table 4.4: Lepton identification requirements for stubless muons. For formulas with mixed units, $\mathrm{E}_{T}$ is measured in units of $\mathrm{GeV}$ and $p_{T}$ is measured in units of $\mathrm{GeV} / c$. 


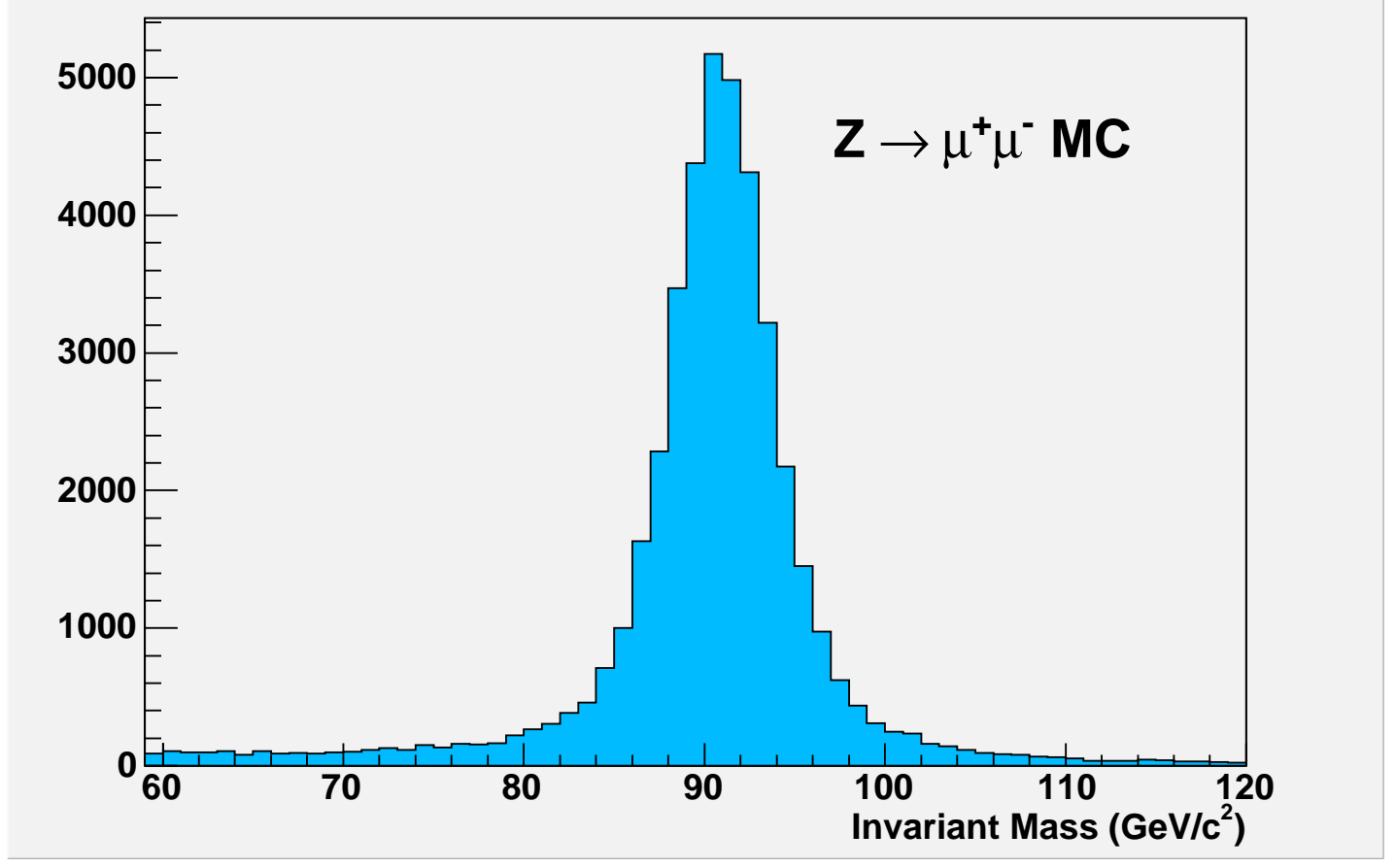

Figure 4.1: Invariant mass distribution for $Z \rightarrow \mu^{+} \mu^{-}$events peaked at the $Z$ boson mass of $91 \mathrm{GeV} / c^{2}$.

$\epsilon_{I D}$ is approximately equal to the number of probe leptons passing all the ID cuts divided by the total number of probe leptons. This is only an approximate value since, even though the background is small, it still must be taken into account. In addition to $\mathrm{Z}$ decays which produce high- $p_{T}$ leptons, $\mathrm{J} / \psi(c \bar{c}$ meson with a mass of $\left.3.1 \mathrm{GeV} / c^{2}\right)$ and $\Upsilon\left(b \bar{b}\right.$ meson with a mass of $\left.9.5 \mathrm{GeV} / c^{2}\right)$ decays may also be used to study the $\epsilon_{I D}$ of lower $p_{T}$ leptons. Lepton ID efficiencies are measured separately for different lepton types and $\mathrm{p}_{T}$ ranges [47, 48, 49, 50, 51, 52, 53]. 


\subsection{Jets Misidentified as Leptons}

As described in Sec. 2.6, a jet is the result of a quark or gluon which undergoes hadronization. Depending on the components within the jet, there can be several identified tracks in addition to a calorimeter cluster. Many of the cuts described in Sec. 4.3 are designed to differentiate electrons and muons from jets. Since jet production rates are much higher than lepton production rates, even if only a small fraction of jets behave identically to electrons or muons, they could significantly reduce the purity of the lepton samples. Jets can be misidentified as electrons, for example, if there is a $\pi^{0}$ overlapping a track, and can be misidentified as muons by jets that "punch-through" the calorimeter.

The probability that a jet is misidentified as a lepton $\left(P_{j \rightarrow \ell}\right)$ is calculated by the following formula:

$$
P_{j \rightarrow \ell}=\frac{\# \text { Jet candidates passing lepton ID requirements }}{\# \text { Jet candidates }}
$$

where the jet candidate sample should be a sample with a high purity of unbiased jets. For this purpose, four triggers are used: Jet20, Jet50, Jet70, and Jet100, where the number indicates the energy threshold needed to fire the trigger. For instance, the Jet50 trigger requires at least one EM calorimeter cluster with $E_{T}>50 \mathrm{GeV}$. Since the $E_{T}$ requirement biases the highest energy calorimeter cluster in the event, this cluster is removed from the jet candidate sample. In addition, the calorimeter clusters may be pre-classified as electrons and not jets. Therefore, to increase the jet purity of the sample, events with $\mathbb{E}_{T}>15 \mathrm{GeV}$ are rejected since they may be a result of $W \rightarrow \ell \nu$ decays, and possible $Z$ events are removed if there are two clusters, each associated with a track, where the two-track invariant mass is consistent with the $Z$ mass, i.e. between 76 and $106 \mathrm{GeV} / c^{2}$. The denominator of the $P_{j \rightarrow \ell}$ calculation is slightly different for 
electrons and muons. For calculating the probability that a jet is misidentified as an electron, the denominator jet candidate is required to have $E_{T}>4 \mathrm{GeV}$. In calculating the probability that a jet is misidentified as a muon, the denominator jet candidate is required to have a track with $p_{T}>4 \mathrm{GeV} / c$ and isolation $<0.1$, as defined in Sec. 4.3.

The lepton ID requirements are then applied to the denominator jet candidates. Those that pass the lepton ID criteria are defined to be jets misidentified as leptons and the numerator of the $P_{j \rightarrow \ell}$ calculation. $P_{j \rightarrow \ell}$ is shown as a function of $E_{T}$ for LCEs in Fig. 4.2 and for TCEs in Fig. 4.3. The colored points correspond to $P_{j \rightarrow \ell}$ as measured using the four different jet samples. The total probabilities (solid black line) are determined by fitting the average value of the four samples, defined in Table 4.5. The dashed black lines, corresponding to $\pm 50 \%$ of the average value, cover most of the variation between the four jet samples and are therefore used as systematic uncertainties (see Sec. 6.4). The rise in $P_{j \rightarrow L C E}$ as a function of $E_{T}$ in Fig. 4.2, is due to the isolation cut, which becomes less stringent as the jet $E_{T}$ increases. On the other hand, $P_{j \rightarrow T C E}$ which is shown in Fig. 4.3, is relatively flat in $E_{T}$, indicating that the additional $E / p$ and CES based cuts are better at removing jet candidates that are misidentified as central electrons at higher $E_{T} \cdot P_{j \rightarrow L C E}$ is approximately 3 times larger than $P_{j \rightarrow T C E}$

The probability that a jet is misidentified as a stand-alone plug electron depends not only on $E_{T}$ but also on $|\eta|$. Therefore, this probability was calculated separately for "low-eta" $(1.1<|\eta|<1.3)$, where the tracks include COT hits, and for "high-eta" $(|\eta|>1.3)$, where the tracks do not include COT hits. This probability is shown for low-eta in Fig. 4.4 and for high-eta in Fig. 4.5. $P_{j \rightarrow \text { phoenix }}$, shown in Fig. 4.6, does not depend on $|\eta|$. For each type of plug electron, 


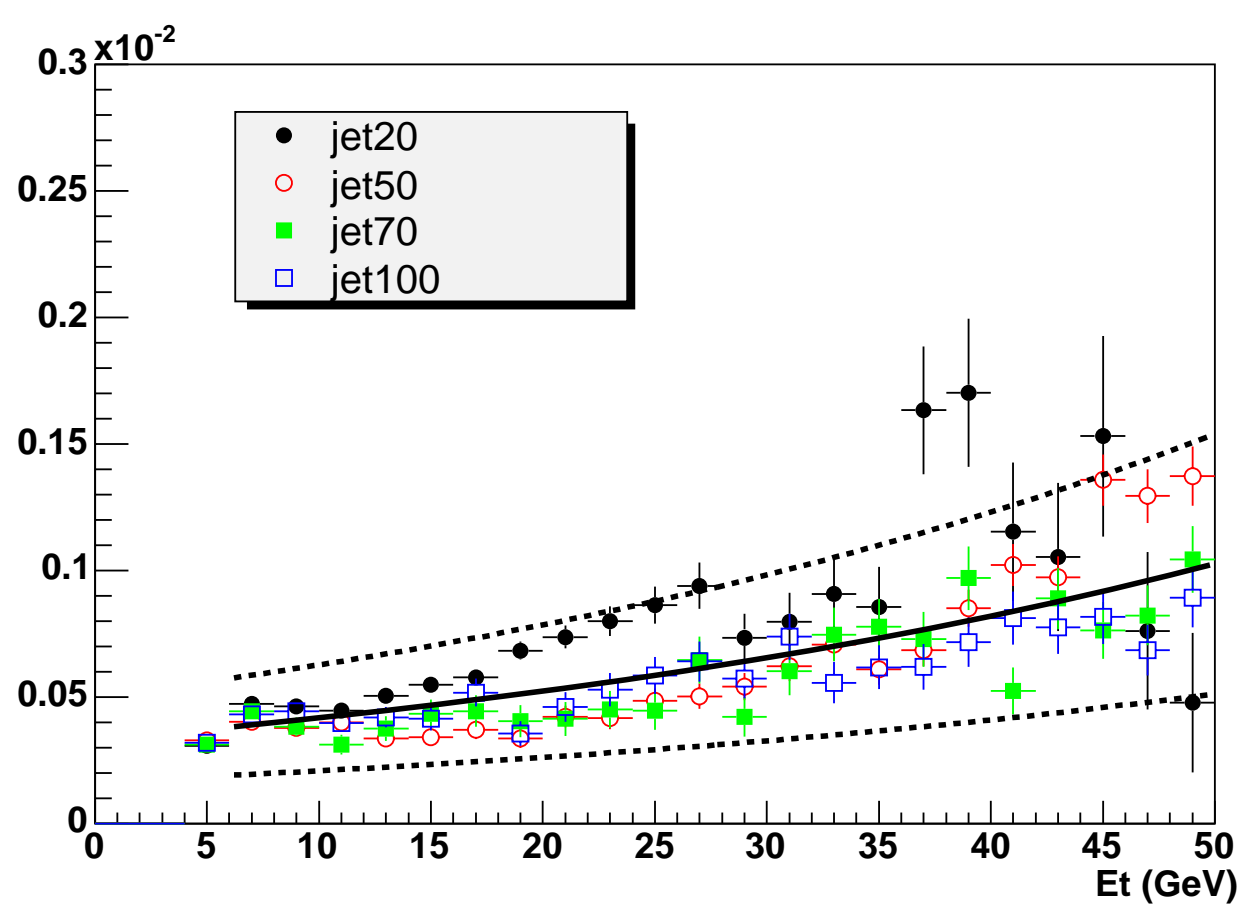

Figure 4.2: Probability for a CEM cluster with $E_{T}>4 \mathrm{GeV}$ to be misidentified as a loose central electron (LCE), as measured in four samples with high concentrations of jets.

the $P_{j \rightarrow \ell}$ 's are parameterized by a straight line, defined in Table 4.5 , where the systematic uncertainties are determined using the same method as for central electrons.

$P_{j \rightarrow \mu}$ is shown for CMUP muons in Fig. 4.7, for CMX muons in Fig. 4.8, and for CMIO muons in Fig. 4.9. For each category of muon, the probability increases as a function of $p_{T}$ because higher energy jet showers are more likely to reach the muon subdetectors. $P_{j \rightarrow \mu}>P_{j \rightarrow e}$ since the jet candidate definition is more selective for muons than it is for electrons due to the track and isolation requirements. This probability is smaller for CMUP and CMX muons than it is for CMIO muons due to the stub requirement. 


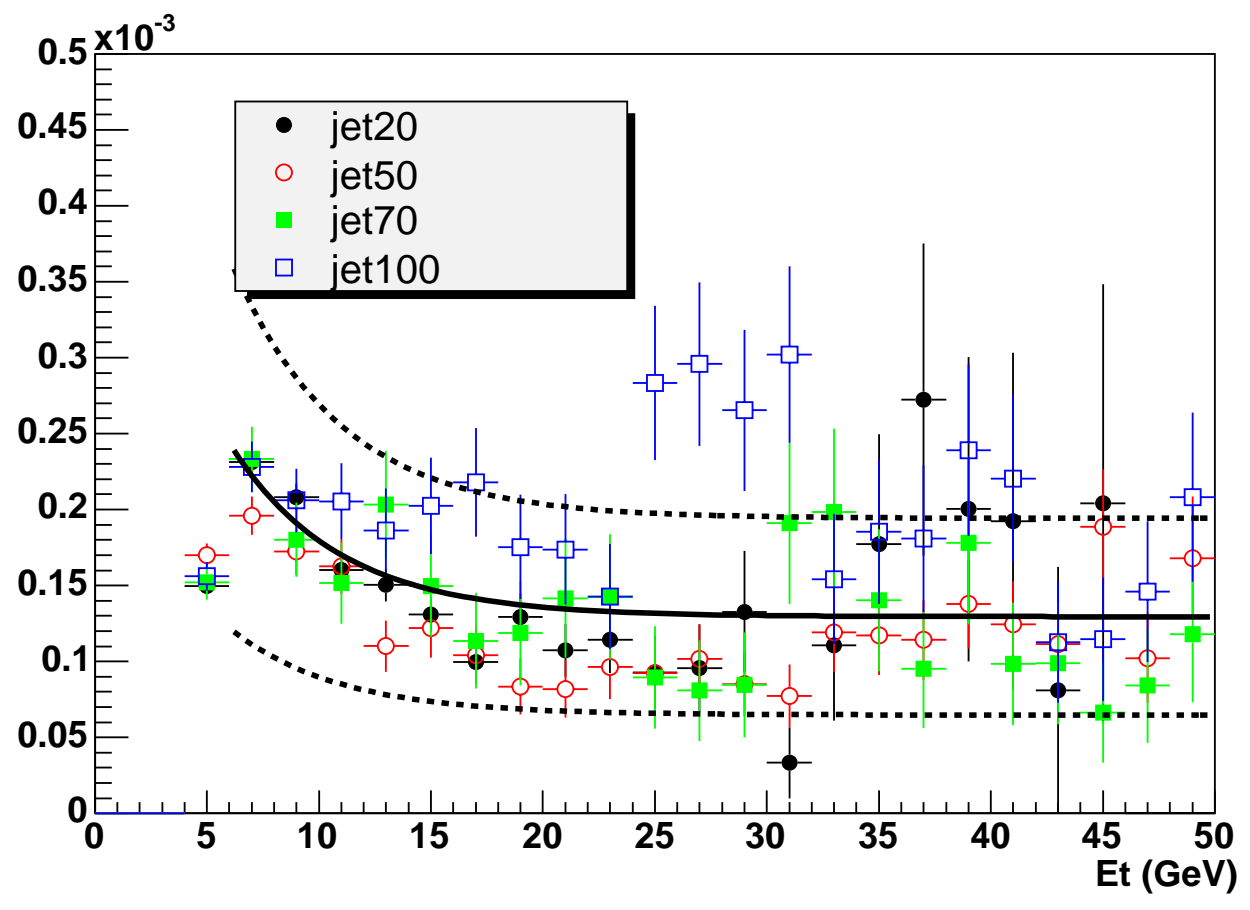

Figure 4.3: Probability for a CEM cluster with $E_{T}>4 \mathrm{GeV}$ to be misidentified as a tight central electron (TCE), as measured in four samples with high concentrations of jets. 


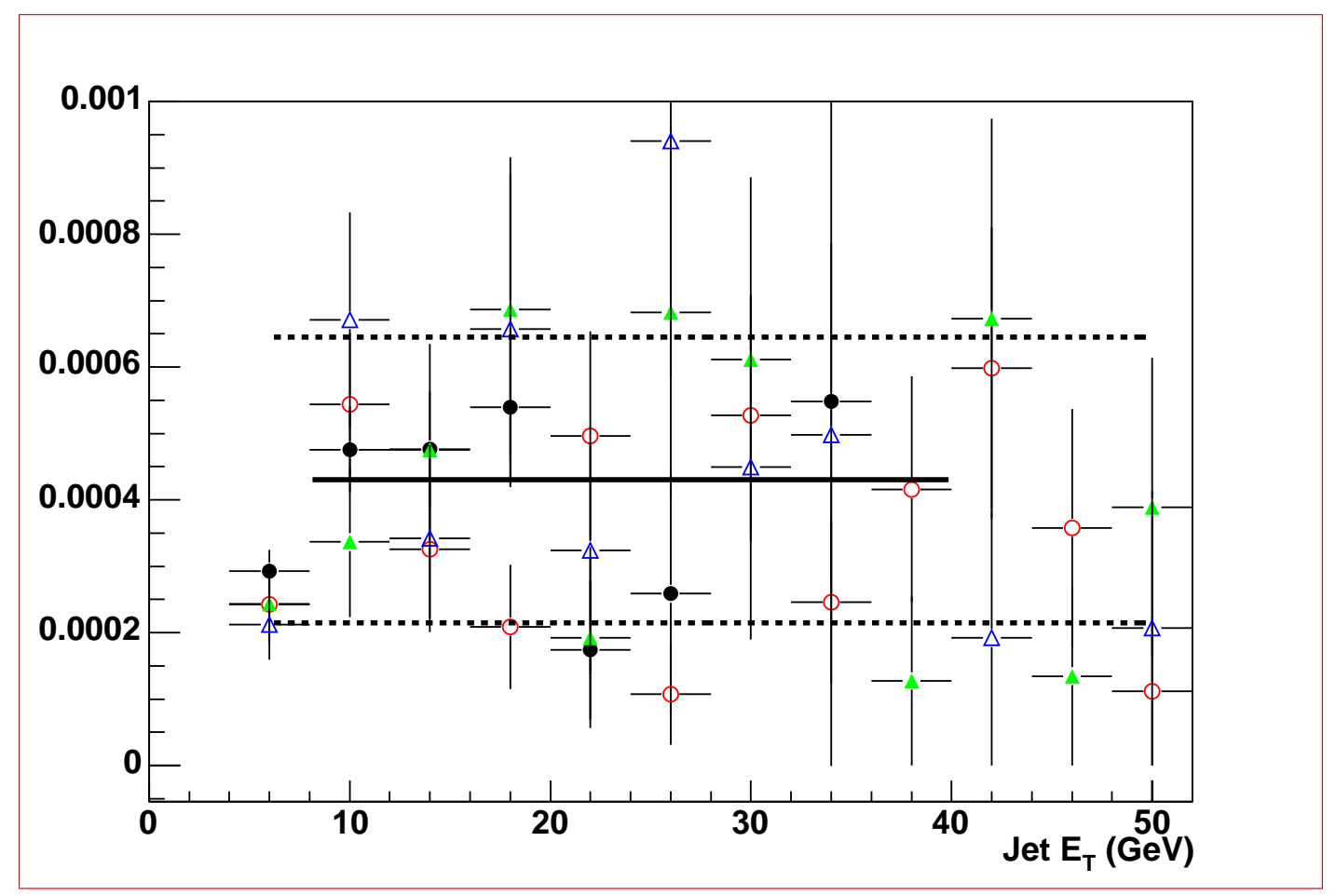

Figure 4.4: Probability for a PEM cluster with $E_{T}>8 \mathrm{GeV}$ to be misidentified as a stand-alone plug electron with $1.1<|\eta|<1.3$, as measured in four samples with high concentrations of jets. 


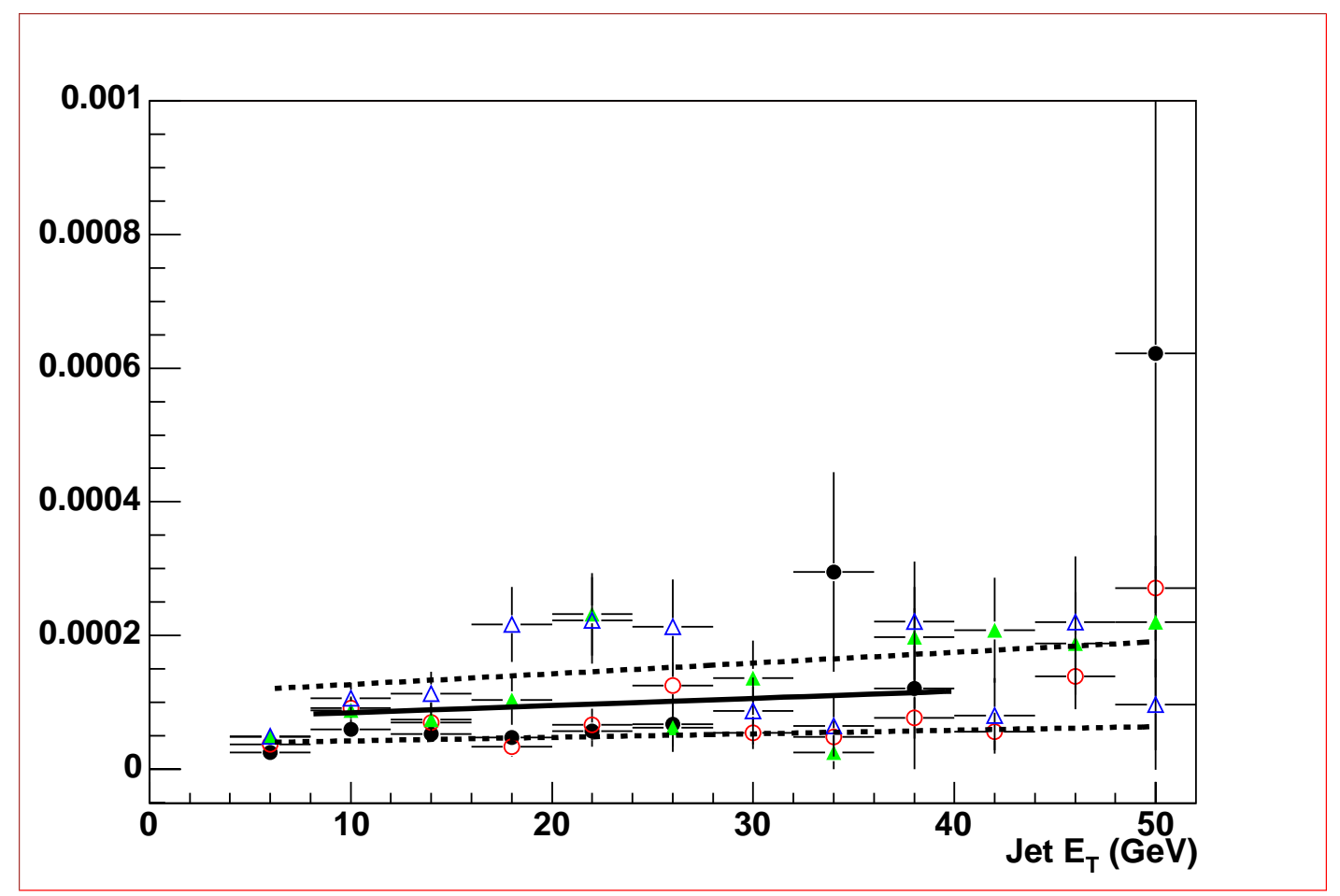

Figure 4.5: Probability for a PEM cluster with $E_{T}>8 \mathrm{GeV}$ to be misidentified as a stand-alone plug electron with $|\eta|>1.3$, as measured in four samples with high concentrations of jets. 


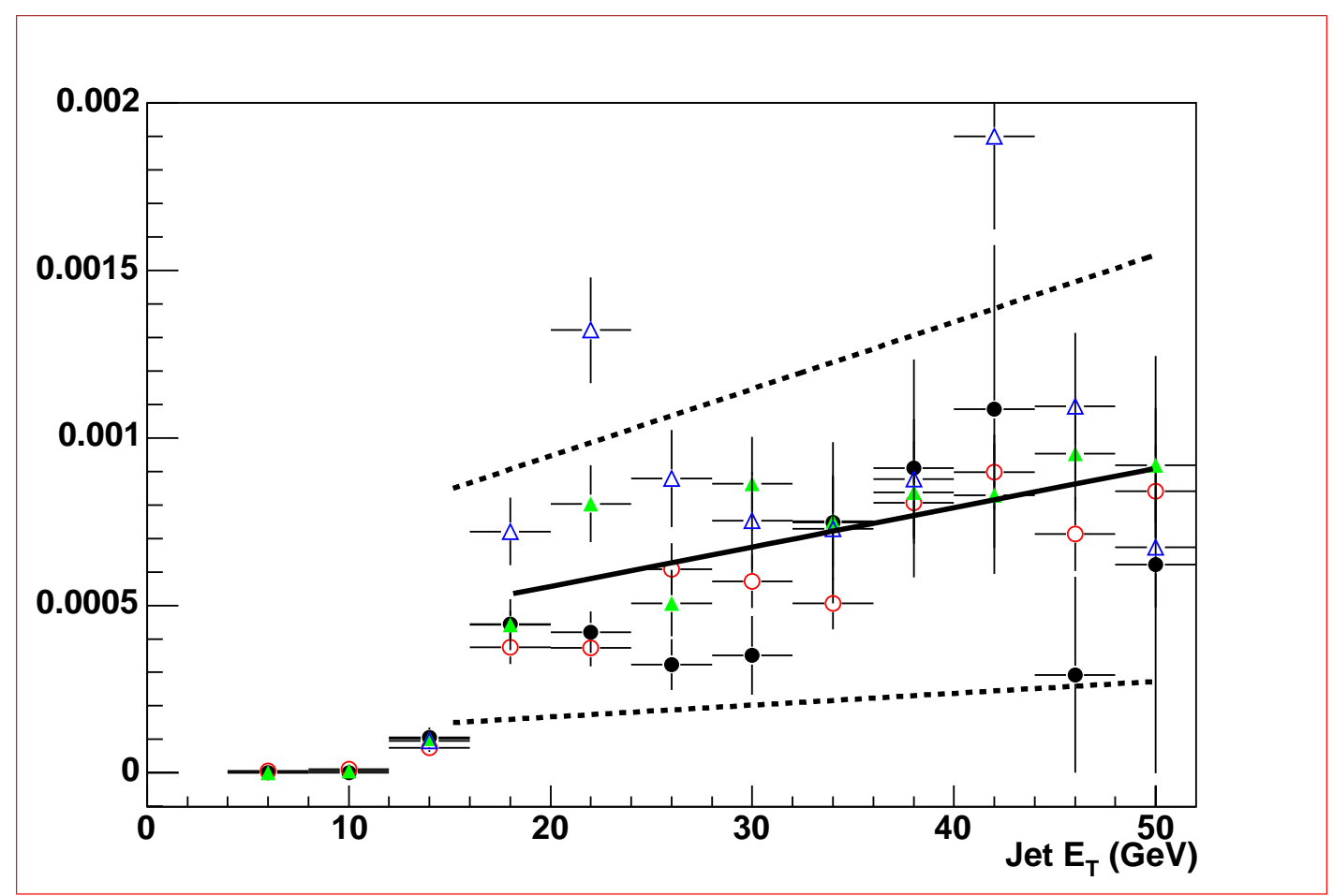

Figure 4.6: Probability for a PEM cluster with $E_{T}>15 \mathrm{GeV}$ to be misidentified as a phoenix electron, as measured in four samples with high concentrations of jets. 


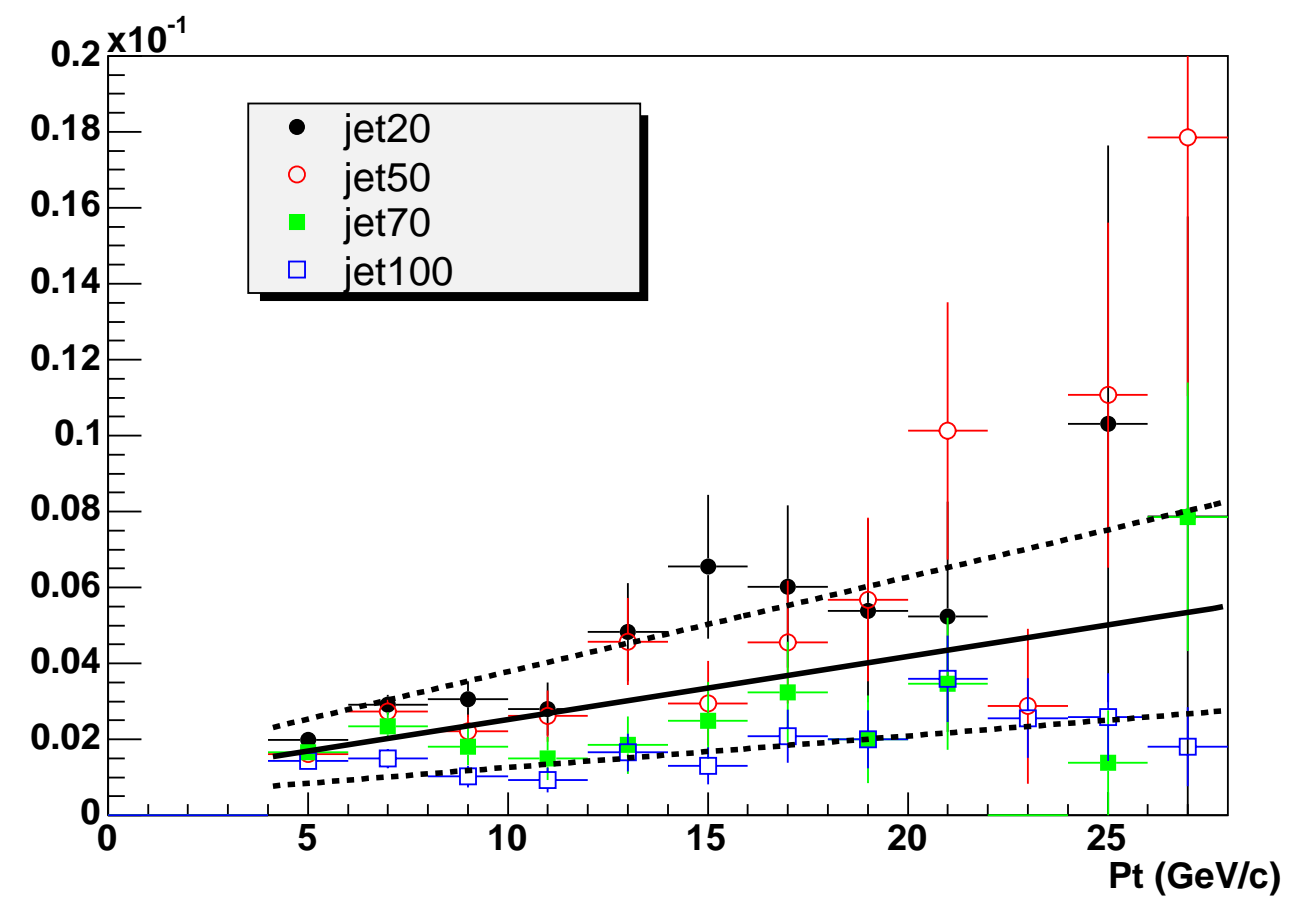

Figure 4.7: Probability for an isolated track with $p_{T}>4 \mathrm{GeV} / c$ to be misidentified as a CMUP muon, as measured in four samples with high concentrations of jets. 


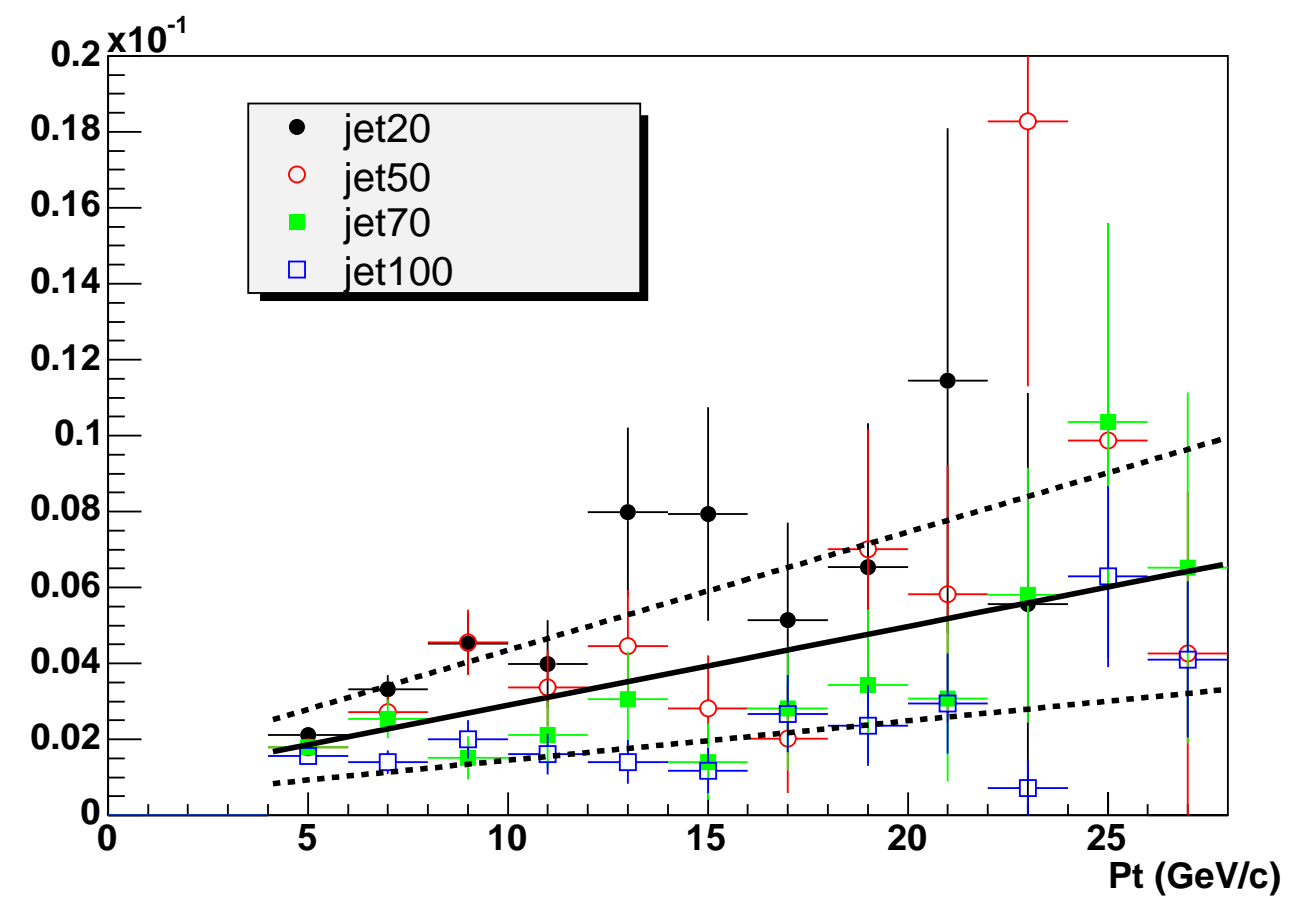

Figure 4.8: Probability for an isolated track with $p_{T}>4 \mathrm{GeV} / c$ to be misidentified as a CMX muon, as measured in four samples with high concentrations of jets. 


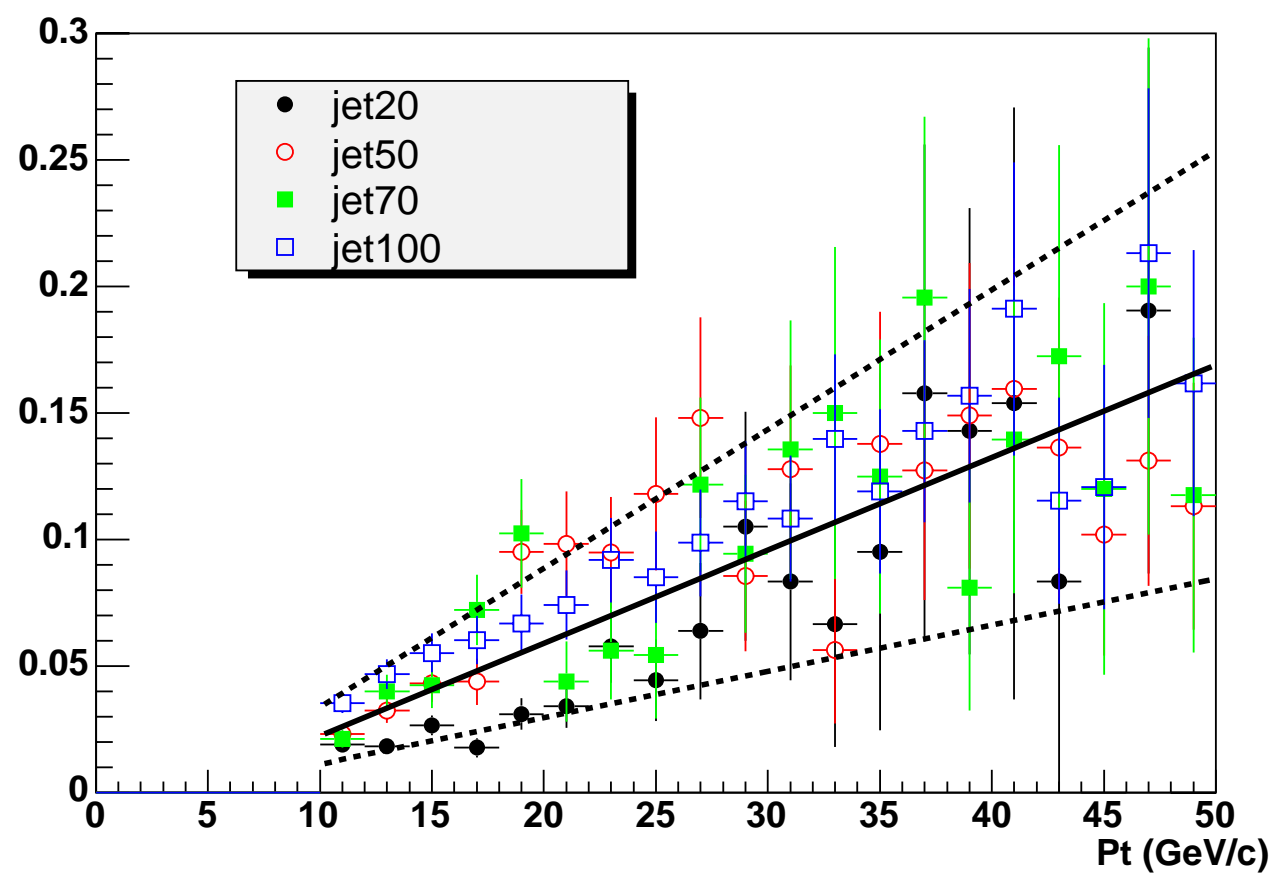

Figure 4.9: Probability for an isolated track with $p_{T}>10 \mathrm{GeV} / c$ to be misidentified as a CMIO muon, as measured in four samples with high concentrations of jets.

\subsection{Photon Conversion Identification}

Photon conversions occur when a photon converts into an electron and positron pair through interaction with material, as shown in Fig. 4.10. To identify these events, the following information can be used: the tracks belonging to the electron and positron should have opposite sign; since momentum transfer is small, most conversion pairs will initially travel in the same direction as the parent photon; and since off-shell photons tend to have small mass, the invariant mass of the two tracks should be approximately zero [72]. Electrons are first identified using the selection criteria described in Sec. 4.3.1, then a second track is sought 


\begin{tabular}{lllll}
\hline Lepton Type & Function & $a$ & $b$ & $c$ \\
\hline LCE & $e^{b+c E_{T}}$ & - & -7.98 & 0.022 \\
TCE & $a+e^{b+c E_{T}}$ & $1.3 \times 10^{-4}$ & -7.94 & -0.194 \\
\hline Plug stand-alone, $|\eta|<1.3$ & $a+b E_{T}$ & $4.3 \times 10^{-4}$ & - & - \\
Plug stand-alone, $|\eta|>1.3$ & $a+b E_{T}$ & $7.4 \times 10^{-5}$ & $1.1 \times 10^{-6}$ & - \\
Phoenix & $a+b E_{T}$ & $3.2 \times 10^{-4}$ & $1.2 \times 10^{-5}$ & - \\
\hline CMUP muon & $a+b p_{T}$ & $8.6 \times 10^{-4}$ & $1.7 \times 10^{-4}$ & - \\
CMX muon & $a+b p_{T}$ & $8.2 \times 10^{-4}$ & $2.0 \times 10^{-4}$ & - \\
CMIO muon & $a+b p_{T}$ & $-1.4 \times 10^{-2}$ & $3.7 \times 10^{-3}$ & - \\
\hline
\end{tabular}

Table 4.5: Parameterizations and fit values for the probabilities for jet candidates to be misidentified as leptons, for each lepton type. $\mathrm{E}_{T}$ and $p_{T}$ are measured in units of $\mathrm{GeV}$ and $\mathrm{GeV} / c$, respectively.

for which possesses the opposite sign and satisfies the photon conversion requirements, identifying the electron as part of a photon conversion. For CDF Run II, the standard photon conversion requirements are: $\mathrm{D}_{x y}<0.2 \mathrm{~cm}$, where $\mathrm{D}_{x y}$ is the distance between the two tracks in the transverse plane when the tracks are parallel, and $\Delta \cot \theta<0.4$. If the electron is tagged as coming from a photon conversion, it is rejected. This requirement is only applied to central electrons where the tracking reconstruction is optimal.

\subsection{Cosmic Ray Muon Identification}

Not all particles traversing the detector originate from proton-antiproton collisions. For instance, a constant flux of neutrinos come from the sun, but are virtually undetectable since they are not charged, have very little mass, and only 


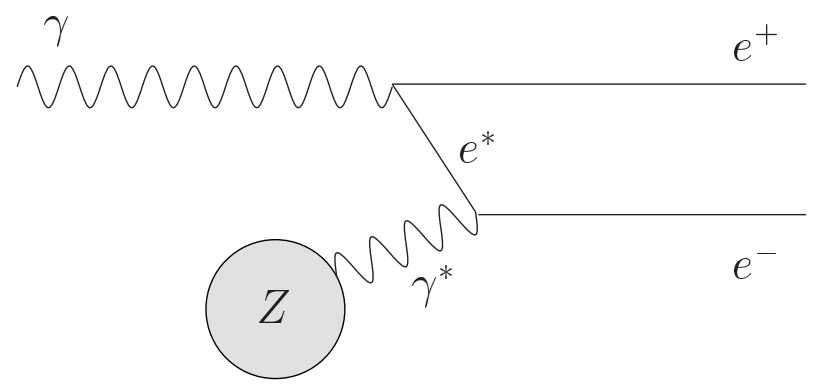

Figure 4.10: Photon conversion $\left(\gamma \rightarrow e^{+} e^{-}\right)$in the presence of an atomic nucleus (Z).

interact through the weak force. On the other hand, muons from cosmic rays can be detected. In order to distinguish between cosmic ray muons and muons coming from a primary vertex, two quantities are used: the direction of flight; and the time of incidence $\left(t_{0}\right)$, the difference in time between the beam collision and the time the track passes the primary vertex. Since the CDF software expects particles to originate from a primary vertex, in general only one track will be reconstructed traveling outward. To identify cosmic ray muons the outgoing track is matched with COT hits on the opposite side of the beamline that are consistent with a particle traveling toward the primary vertex. If this combination of a single track plus COT hits is consistent with the path of a single particle, then it is identified as a cosmic ray muon. In addition, if the acollinearity between a muon and any other track is greater than 3.1 radians, or $2.4^{\circ}$ from being back-to-back, the muon is identified as a cosmic ray muon. 


\section{CHAPTER 5}

\section{Simulated and CDF Run II Data Collections}

In this chapter, the process of selecting CDF events to be used for validation and searching for new physics is described. The simulated data samples created by the Monte Carlo (MC) event generators are also described.

\subsection{R-Parity Violating Supersymmetric Event Simulation}

- What will $R_{p}$ SUSY events look like within the CDF II detector?

- What is the best way to identify these events?

- What regions of the mSUGRA parameter space is this analysis sensitive to?

- How large is the deviation, if any, from the standard model?

To answer these questions, an accurate representation of $\ell_{p}$ SUSY events is needed. This representation is achieved by first determining the production cross sections for SUSY particle pair production in proton antiproton collisions. The PROSPINO2 [26] program is used since it calculates the next-to-leading order (NLO) cross section, which is more precise than the leading order (LO) cross section used by other programs. Second, the spectrum of SUSY masses and couplings are calculated by ISAJET version 7.51 [30], which provides a detailed solution of 
the SUSY renormalization group equations. Third, events are generated using a Monte Carlo (MC) technique with PYTHIA version 6.216 [44] using the previously determined masses and couplings as input. PYTHIA models the interactions of all the particles in the event from the initial collision down to the final state according to input from the user. For simplicity, since chargino-neutralino and chargino-chargino production dominate the inclusive SUSY pair production cross section, they are the only ones generated. In addition all $R_{p}$ decays are turned off except for $\tilde{\chi}_{1}^{0} \rightarrow e^{+} e^{-} \nu_{\mu}+$ charged conjugate (c.c.) and $\tilde{\chi}_{1}^{0} \rightarrow e^{+} \mu^{-} \nu_{e}+$ c.c. for $\lambda_{121}$ couplings, and $\tilde{\chi}_{1}^{0} \rightarrow \mu^{+} \mu^{-} \nu_{e}+$ c.c. and $\tilde{\chi}_{1}^{0} \rightarrow \mu^{+} e^{-} \nu_{\mu}+$ c.c. for $\lambda_{122}$ couplings. Fourth, the events are reconstructed using the full CDF simulation package.

The points in the mSUGRA parameter space are selected based on previous searches for $\not_{p}$ SUSY $[57,58]$. Enough points are needed to cover the region starting from the LEP limits until the signal becomes too small for any chance of detection. The most significant parameter is $M_{1 / 2}$, which affects the mass of the lightest neutralino $\left(\tilde{\chi}_{1}^{0}\right)$ along with the masses of the other supersymmetric fermions. As $M_{1 / 2}$ increases, so does the $\tilde{\chi}_{1}^{0}$ mass, while the production cross section decreases. Signal MC samples with 20,000 events each are generated for the $\lambda_{121}$ and $\lambda_{122}$ couplings, both signs of $\mu$, and for several values of $M_{1 / 2}$ differing by $10 \mathrm{GeV} / c^{2}$ with an additional point such that $\tilde{\chi}_{1}^{0}$ is below the LEP limit of $\sim 53 \mathrm{GeV} / c^{2}$. The selected mSUGRA points, resulting $\tilde{\chi}_{1}^{0}$ and $\tilde{\chi}_{1}^{ \pm}$masses, and the predicted NLO production cross sections, are listed in Table 5.1.

The generated properties of the $\tilde{\chi}_{1}^{0}$ and its decay products can be read directly from PYTHIA. These generated variables are slightly different from the reconstructed variables, which are affected by the detector coverage, detector resolution, and particle overlap. The two most interesting variables are the trans- 
verse momentum $\left(\mathrm{p}_{T}\right)$ and pseudorapidity $(\eta)$, since they have a big impact on the acceptance, the percentage of the events that the detector reconstructs. The generated $\mathrm{p}_{T}$ distributions are shown in Fig. 5.1 for the four leptons originating from the $\tilde{\chi}_{1}^{0}$ for a representative point in SUSY parameter space. Only the leptons from the $\tilde{\chi}_{1}^{0}$ decay and not from the "cascade" decays are shown, since the number and momentum of the leptons from the cascade decays may be model dependent. Three out of the four leptons have a mean $p_{T}$ in the high-range (> $20 \mathrm{GeV} / c$ ) while the fourth has a mean $p_{T}$ of $12 \mathrm{GeV} / c$. The generated $\eta$ distributions are displayed in Fig. 5.2 for the same events. Electrons are identified out to $|\eta|<2$ while muons are identified out to $|\eta|<1$. Leptons with large $\mathrm{p}_{T}$ and $\eta$ in the central region of the calorimeter are the easiest to reconstruct and have smaller backgrounds. As the $\tilde{\chi}_{1}^{0}$ mass increases, so do the lepton $\mathrm{p}_{T}$ and detector acceptance. The two figures also demonstrate how higher $\mathrm{p}_{T}$ leptons have a narrower distribution in $\eta$.

\subsection{Data Collection}

Based on the $\mathrm{p}_{T}$ distribution of the leading lepton in Fig. 5.1, the choice was made to use the inclusive high- $p_{T}$ trigger. There are three high- $p_{T}$ trigger paths: one for central electrons, one for CMUP muons, and one for CMX muons that require an electron (muon) with $\mathrm{E}_{T}\left(\mathrm{p}_{T}\right)>18 \mathrm{GeV}(\mathrm{GeV} / c)$. The full trigger paths are listed in Table 5.2. The events that pass this trigger form the high$p_{T}$ electron dataset while the events that pass the CMUP and CMX triggers are combined to form a single high- $p_{T}$ muon dataset. These datasets include a total of $346 \mathrm{pb}^{-1}$ of data collected between March 2002 and August 2004.

The high- $p_{T}$ electron and muon datasets are combined to form the final dataset used in this analysis. Events with multiple leptons may appear in both of the 
datasets, introducing the possibility of double-counting. In the cases where two events with the same run and event numbers are present, one of those events is removed. All events are required to meet good run requirements for electrons and muons. Since events with plug electrons require SVX subdetector information, the dataset is separated into two categories: runs that meet the good run requirements for the silicon microstrip detectors, corresponding to $318 \mathrm{pb}^{-1}$; and runs that do not, corresponding to $28 \mathrm{pb}^{-1}$.

\subsection{Background MC Samples}

Some people may remember a children's book titled "Where's Waldo?" [59] in which the object is to find Waldo among the detailed scene drawn on the page. Even though Waldo wore bright clothes and glasses, this task was not necessarily easy due to all the other objects and people appearing in the scene. To make it even more difficult, some of the people appearing in the scene would wear clothes very similar to Waldo's. The same idea can be applied to particle physics searches where the goal is to distinguish a particular process from standard model (SM) processes. These SM processes are referred to as backgrounds. What makes particle physics searches more difficult is the uncertainty of whether the signal process even exists in the first place. In order to claim with any certainty that the signal process exists, one must understand the backgrounds.

In this search, the signature from $R_{p}$ SUSY decay is events with multiple leptons and the backgrounds are SM processes that also result in multiple leptons. Multi-lepton backgrounds come from the following sources: Drell-Yan (DY), misidentified jets, photon conversions, diboson production, $t \bar{t}$ production, and heavy flavor production. Except for those due to misidentified jets, the backgrounds are estimated using MC simulation. Together, the backgrounds will be 
used in order to make predictions on the number of events in control samples and signal samples containing the $k_{p}$ SUSY signature.

In order to compare the background expectation with the observed events in data, the effective luminosity of each MC sample must be known. The luminosity, $\mathcal{L}$, can be determined by the cross section $(\sigma)$, branching ratio $(\mathrm{BR})$, and the number of events $(\mathrm{N})$ :

$$
N=\mathcal{L} \times \sigma \times B R
$$

In some cases, decay products are forced to lie below a certain $|\eta|$ value or have a minimum amount of transverse momentum. These restrictions allow for the creation of larger luminosity samples with less generated events as long as they do not affect the event acceptance.

The datasets which are used to calculate the backgrounds are described in more detail below and information on the simulated background samples is provided in Table 5.3. Additional information on computing the background prediction is provided in Sec. 5.4.

\subsubsection{Drell-Yan Background}

DY [60] is the process $q \bar{q} \rightarrow Z / \gamma^{*} \rightarrow \ell^{+} \ell^{-}$, where $\ell$ corresponds to a lepton of any flavor. This process produces the largest number of event with high- $p_{T}$ leptons. DY is simulated by one large dataset corresponding to $7,000 \mathrm{pb}^{-1}$ produced by the PYTHIA generator. Only events where the dilepton invariant mass is greater than $5 \mathrm{GeV} / c^{2}$ are produced. The leading order (LO) cross section is obtained directly from PYTHIA, which is multiplied by a $k$ factor of 1.4 to correct $\sigma$ for higher order terms [61]. 


\subsubsection{Backgrounds From Misidentified Jets}

Misidentified jets can be a significant background due to the large jet production cross section. This background is defined and determined from data using the probabilities for a jet to be misidentified as a lepton $\left(P_{j \rightarrow \ell}\right)$, defined in Sec. 4.5.

To estimate the number of events with $n$ leptons in the final state in which a jet has been misidentified as a lepton, $P_{j \rightarrow \ell}$ is applied to events containing $n-1$ leptons. Since $P_{j \rightarrow \ell}$ is much smaller than the number of jet candidates in each event and the $n$ lepton sample may already contain jets misidentified as leptons, this procedure automatically includes events in which multiple jets have been misidentified as leptons.

The background calculation is done in three steps according to the formula:

$$
N_{\text {background events }}=\sum_{\ell}\left(\# \text { jet candidates } \times P_{j \rightarrow \ell}\right),
$$

where $\ell$ represents the different types of identified leptons. First, a sample of events is collected with at least one jet candidate, either an electromagnetic cluster with $E_{T}>4 \mathrm{GeV}$ or an isolated track with $p_{T}>4 \mathrm{GeV} / c$ depending on whether $\ell$ is an electron or muon. The EM cluster or track can not be associated with an identified electron or muon. Second, the number of misidentified leptons per event is determined by multiplying the total number of jet candidates by the probability for a jet to be misidentified as a lepton for each lepton type. Third, the prediction for each lepton type is added together to get the total number of jets misidentified as leptons in the event.

\subsubsection{Backgrounds From Photon Conversions}

In Section 4.7, a method of identifying photons converting into an electron and positron pair was introduced. This method is not $100 \%$ efficient and therefore a 
fraction of conversion electrons will be left in the signal samples. For example, if one of the conversion electrons has a $p_{T}<1 \mathrm{GeV} / c$, it may not be reconstructed by the tracking system. Since the photon conversion identification criteria requires reconstruction of both tracks, the other electron will not be tagged as originating from a photon conversion.

Photons can be produced through EM radiation or through hadronic decays, i.e. $\pi^{0} \rightarrow \gamma \gamma$. Since hadrons are produced primarily in jets, the latter is included in the estimation of backgrounds with jets misidentified as leptons. EM radiation can occur in the initial state (initial state radiation, or ISR), i.e. one of the quarks within the proton or antiproton radiates a photon, or a photon can be radiated from a final state particle such as an electron or muon (final state radiation, or FSR). Backgrounds including conversion electrons are estimated from $\mathrm{W}+\gamma$, $Z / \gamma^{*}+\gamma$, and $Z / \gamma^{*}+\gamma \gamma \mathrm{MC}$ [62], where the $\gamma$ may be produced through ISR or FSR. For each MC sample, the boson is required to decay leptonically, either into a lepton plus a neutrino for W events or two opposite sign leptons for $Z / \gamma^{*}$ events. If the photon converts and is not identified by the photon conversion requirements, then there will be an additional electron to those already coming from the $\mathrm{W}$ or $\mathrm{Z}$ boson. This background is one of the most dominant for events with three or more leptons.

\subsubsection{Diboson Backgrounds}

Diboson production includes $W W, W Z / \gamma^{*}$, and $Z / \gamma^{*} Z / \gamma^{*}$ events. $W$ bosons can decay into leptons by $W^{ \pm} \rightarrow \ell^{ \pm}+\nu$. $W W$ and $Z / \gamma^{*} Z / \gamma^{*}$ are generated with PYTHIA, while $W Z / \gamma^{*}$ is generated using the MADGRAPH program [63]. 


\subsubsection{Top Quark Background}

Top quarks decay into a $W$ boson and a $b$ quark. Top-antitop pairs may result in final state leptons through leptonic $W$ decay into a lepton plus a neutrino, or through leptonic $b$ quark decays. A sample of $t \bar{t}$ events are generated with PYTHIA using a theoretical cross section of $6.7 \mathrm{pb}$ [64]. This corresponds to a luminosity of $143,000 \mathrm{pb}^{-1}$.

\subsubsection{Heavy Flavor Backgrounds}

Production of $b \bar{b}$ and $c \bar{c}$ events is referred to as heavy flavor production. These quarks hadronize into baryons and mesons that may in turn decay semileptonically, although the resulting leptons tend to be less isolated. Heavy flavor production can occur in three main processes: direct production $(q \bar{q} \rightarrow b \bar{b} / c \bar{c})$, flavor excitation $(g b \rightarrow g b, q b \rightarrow q b$, or $\bar{q} b \rightarrow \bar{q} b+$ c.c. $)$, and gluon splitting $(g \rightarrow$ $b \bar{b} / c \bar{c})$. All three production mechanisms are generated with ISAJET in three different transverse momentum ranges: low $(10-25 \mathrm{GeV} / \mathrm{c})$, medium $(25-50 \mathrm{GeV} / \mathrm{c})$, and high $(>50 \mathrm{GeV} / \mathrm{c})$.

\subsection{Background and Signal Predictions}

Using the background samples described above, we can predict the number of expected events due to the SM or due to hypothetical $\ell_{p}$ SUSY signals for a particular final state. This prediction has already been discussed for backgrounds including misidentified jets in Sec. 5.3.2, and therefore in this section we will focus on estimations from MC simulation.

In principle, making predictions based on MC simulation is very similar to determining the number of events in data: choosing a set of event selection cri- 
teria and counting the number of events that pass. For MC events, we also must multiply the number of events by a "scale factor" to account for differences between the MC and data samples. These scale factors are described below:

Luminosity: The integrated luminosity of each sample $\left(\mathcal{L}_{M C}\right)$ is listed in Table 5.3. The MC events are scaled by $\mathcal{L}_{\text {Data }} / \mathcal{L}_{M C}$.

Trigger Efficiency: The trigger efficiencies listed in Table 5.2 are not included in the MC and have to be applied by hand. The listed efficiencies are for events with one high- $p_{T}$ TCE, CMUP, or CMX lepton $>20 \mathrm{GeV}$. If there is more than one such lepton in a single event, there is a much greater chance for that event to pass the trigger. For two of them, the trigger efficiency $\left(\epsilon_{\text {trig }}\right)$ is determined by:

$$
\epsilon_{\text {trig }}=\epsilon_{\text {trig }}^{1}+\epsilon_{\text {trig }}^{2}-\epsilon_{\text {trig }}^{1} \times \epsilon_{\text {trig }}^{2}
$$

If there are three or more high- $p_{T}$ TCE, CMUP, or CMX leptons, then the trigger efficiency used is $\epsilon_{\text {trig }}=1$. Each MC event that passes the selection criteria is scaled by the appropriate $\epsilon_{\text {trig }}$.

Lepton ID Efficiency: The efficiency for identifying a lepton is different in $\mathrm{MC}$ events than in data events. For each type of lepton and for various $E_{T}$ or $p_{T}$ ranges, a scale factor is determined equal to $\epsilon_{\text {Data }}^{I D} / \epsilon_{M C}^{I D}$. The lepton ID scale factors, listed in Table 5.4, are applied to each identified lepton in the simulated event.

Photon Conversion ID Efficiency: How often a photon converts greatly depends on the amount and position of material in the CDF detector. For this reason, the position and type of material is described in the detector simulation. 
Yet due to the complexity of the detector, there are some differences in the simulation from the actual detector that may lead to misrepresentations in the boson + photon background samples. To account for this, the efficiency to detect photon conversions is studied in both data and MC (see Appendix B). The result of this study is an $E_{T}$ dependent scale factor determined to be approximately $\max \left[\left(1.581-0.0152 \times E_{T}\right), 1\right]$, which is primarily due to track reconstruction at low $p_{T}$. It is only applied to identified electrons, which are not removed by the conversion cuts but can be traced to a photon conversion at generator level.

If all the scale factors except for luminosity are combined, the value ranges from 0.6 to 1.0, mostly depending on the number and types of leptons in the event. The uncertainty on the background and $\ell_{p}$ SUSY predictions will be discussed in Sec. 6.4.

Since the MC samples contain jets within the simulated events, there can be an overlap between the $\mathrm{MC}$ background predictions and the predicted number of events containing misidentified jets. To remove this overlap, we require that the number of identified leptons must be less than or equal to the number of generated leptons in the MC samples. Although it is still possible for an event to pass this requirement by having a lepton which fails the identification criteria and a jet which is misidentified as a lepton simultaneously, the chance of this occurring is very small. 


\begin{tabular}{|c|c|c|c|c|c|c|c|}
\hline \multicolumn{5}{|c|}{ Input Parameters } & \multicolumn{3}{|c|}{ Outputs } \\
\hline$\lambda$ & $\begin{array}{c}\mathrm{M}_{0} \\
\left(\mathrm{GeV} / c^{2}\right)\end{array}$ & $\begin{array}{c}\mathrm{M}_{1 / 2} \\
\left(\mathrm{GeV} / c^{2}\right)\end{array}$ & $\tan \beta$ & $\mu$ & $\begin{array}{c}\mathrm{M} \tilde{\chi}_{1}^{0} \\
\left(\mathrm{GeV} / c^{2}\right)\end{array}$ & $\begin{array}{c}\mathrm{M} \tilde{\chi}_{1}^{ \pm} \\
\left(\mathrm{GeV} / c^{2}\right)\end{array}$ & $\begin{array}{c}\mathrm{NLO} \sigma \\
(\mathrm{pb})\end{array}$ \\
\hline 121,122 & 250 & 110 & 5 & $<0$ & 44.28 & 83.45 & 4.29 \\
\hline 121 & 250 & 200 & 5 & $<0$ & 80.11 & 151.45 & 0.322 \\
\hline 121 & 250 & 210 & 5 & $<0$ & 84.09 & 158.98 & 0.251 \\
\hline 121 & 250 & 220 & 5 & $<0$ & 88.07 & 166.81 & 0.197 \\
\hline 121,122 & 250 & 230 & 5 & $<0$ & 92.06 & 174.83 & 0.154 \\
\hline 121,122 & 250 & 240 & 5 & $<0$ & 96.02 & 182.27 & 0.124 \\
\hline 121,122 & 250 & 250 & 5 & $<0$ & 100.03 & 190.15 & 0.098 \\
\hline 122 & 250 & 260 & 5 & $<0$ & 104.18 & 198.13 & 0.077 \\
\hline 122 & 250 & 270 & 5 & $<0$ & 108.20 & 205.68 & 0.064 \\
\hline 122 & 250 & 280 & 5 & $<0$ & 112.89 & 213.73 & 0.050 \\
\hline 121,122 & 250 & 130 & 5 & $>0$ & 42.70 & 75.08 & 7.29 \\
\hline 121 & 250 & 230 & 5 & $>0$ & 86.67 & 156.97 & 0.269 \\
\hline 121,122 & 250 & 240 & 5 & $>0$ & 90.80 & 164.65 & 0.213 \\
\hline 121,122 & 250 & 250 & 5 & $>0$ & 95.03 & 173.09 & 0.164 \\
\hline 121,122 & 250 & 260 & 5 & $>0$ & 99.37 & 181.35 & 0.128 \\
\hline 121,122 & 250 & 270 & 5 & $>0$ & 103.53 & 189.12 & 0.101 \\
\hline 121,122 & 250 & 280 & 5 & $>0$ & 107.69 & 197.44 & 0.082 \\
\hline 122 & 250 & 290 & 5 & $>0$ & 112.01 & 205.76 & 0.063 \\
\hline 122 & 250 & 300 & 5 & $>0$ & 116.93 & 213.84 & 0.050 \\
\hline
\end{tabular}

Table 5.1: The mSUGRA input parameters (left) and resulting output quantities (right) for each MC sample generated for this analysis. $A_{0}=0$ for all points. Masses are calculated with ISAJET v7.51. The last column is the NLO $\sigma\left(q \bar{q} \rightarrow \tilde{\chi}_{2}^{0} \tilde{\chi}_{1}^{ \pm}\right)+\sigma\left(q \bar{q}^{\prime} \rightarrow \tilde{\chi}_{1}^{ \pm} \tilde{\chi}_{1}^{\mp}\right)$ as determined by PROSPINO2. 

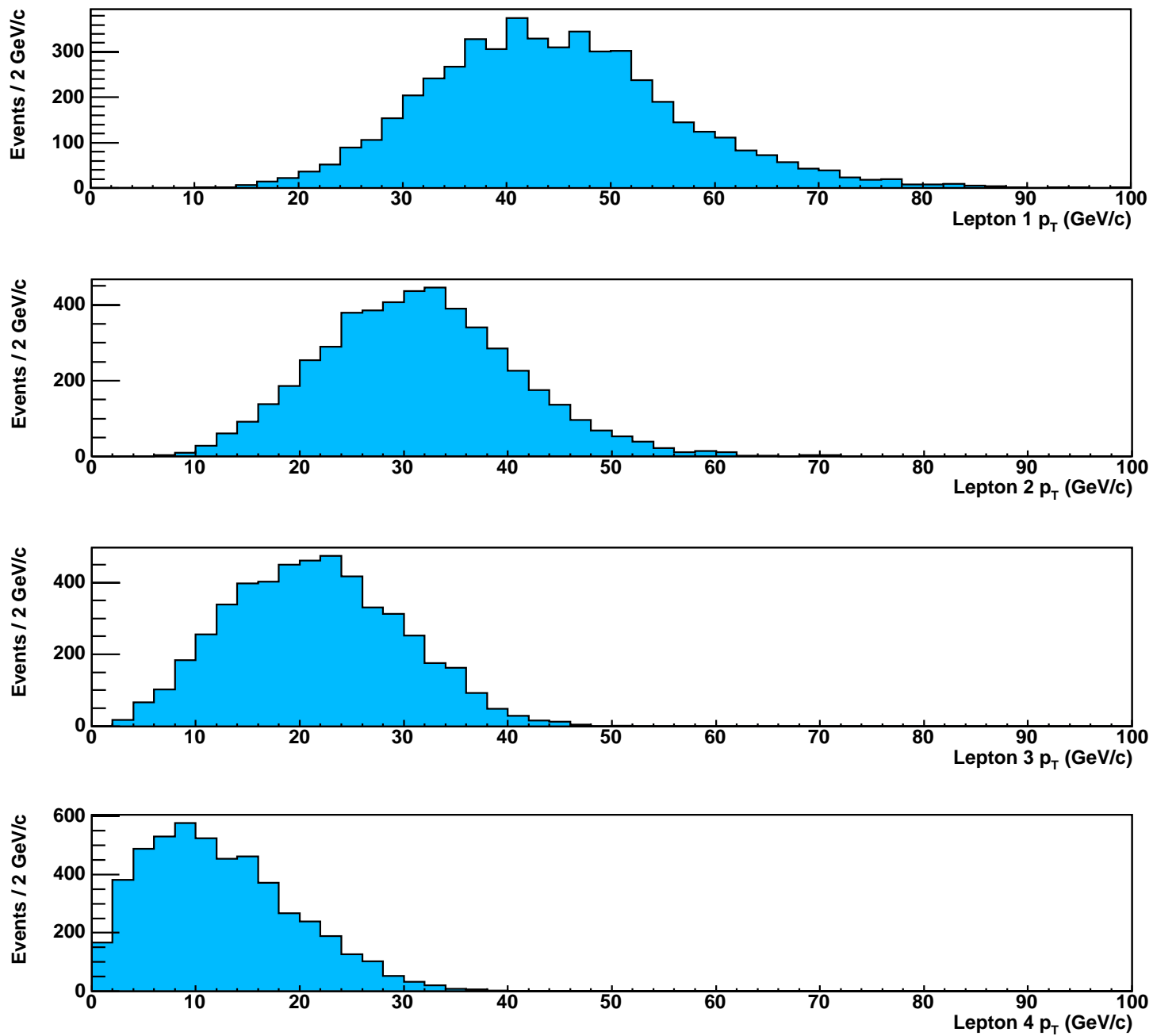

Figure 5.1: Generated $\mathrm{p}_{T}$ distribution of the four leptons from the $\tilde{\chi}_{1}^{0}$ decays for $\tilde{\chi}_{1}^{0}$ mass $=99.4 \mathrm{GeV} / c^{2}$. In each event, the leptons are ordered in $p_{T}$ with largest plotted on top and smallest plotted on bottom. The mean of the distributions are: $45,32,21$, and $12 \mathrm{GeV} / c$. 

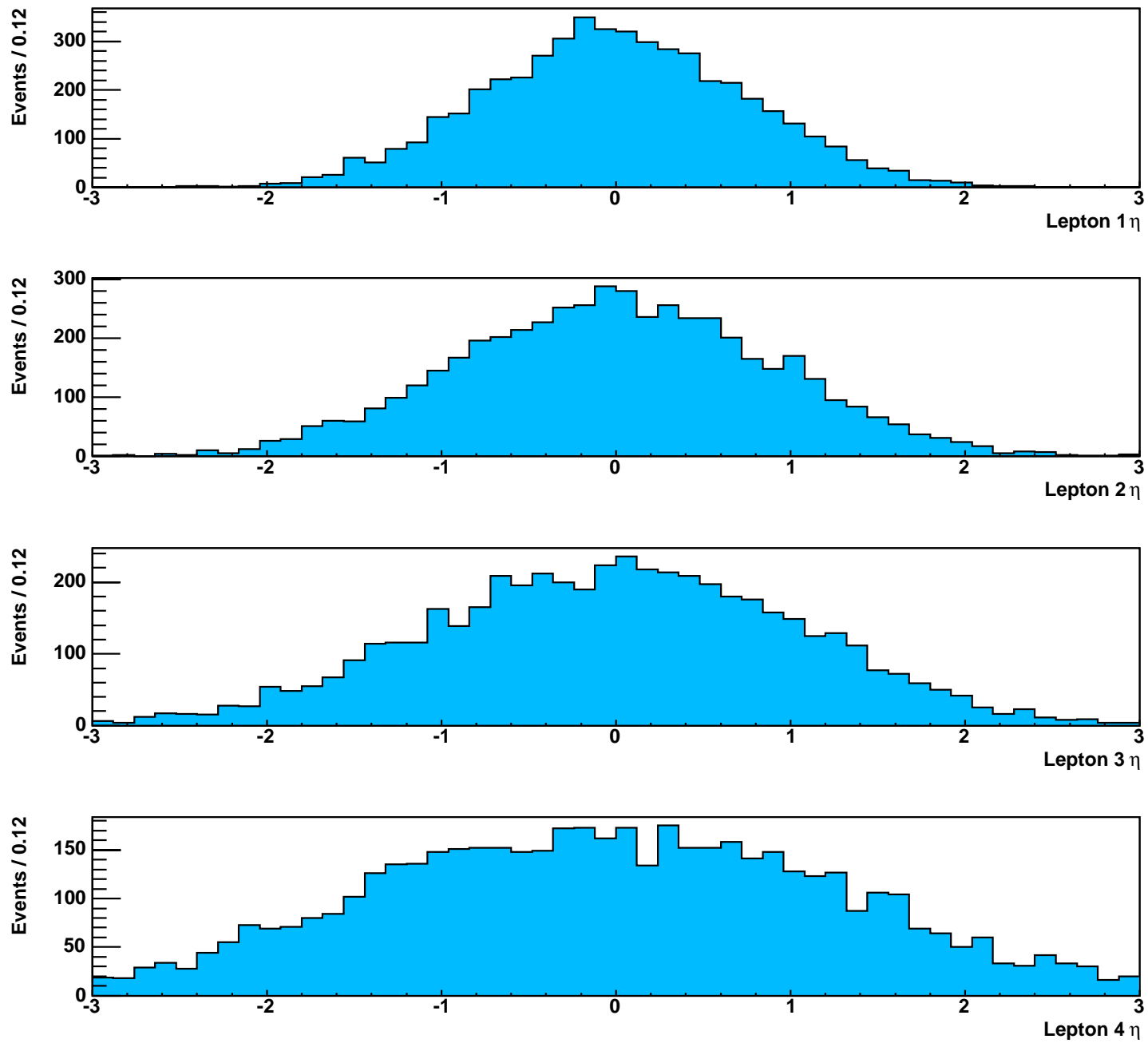

Figure 5.2: Generated $\eta$ distribution of the four leptons from the $\tilde{\chi}_{1}^{0}$ decays for $\tilde{\chi}_{1}^{0}$ mass $=99.4 \mathrm{GeV} / c^{2}$. In each event, the leptons are ordered in $p_{T}$ with largest plotted on top and smallest plotted on bottom. 


\begin{tabular}{cccc}
\hline & CEM & CMUP & CMX \\
\hline L-1 & $8 \mathrm{GeV}$ CEM Tower & $6 \mathrm{GeV}$ CMU Stub & 6 GeV CMX Stub \\
& $8 \mathrm{GeV}$ XFT Track & $4 \mathrm{GeV}$ XFT Track & $4 \mathrm{GeV}$ XFT Track \\
\hline L-2 & $16 \mathrm{GeV}$ CEM Cluster & $8 \mathrm{GeV}$ XFT Track & - \\
\hline L-3 & $18 \mathrm{GeV}$ CEM Electron & $18 \mathrm{GeV}$ CMUP Muon & $18 \mathrm{GeV}$ CMX Muon \\
\hline$\epsilon_{\text {trig }}$ & $(85.2-96.5 \pm 0.1) \%$ & $(90.78 \pm 0.47) \%$ & $(96.49 \pm 0.40) \%$ \\
\hline
\end{tabular}

Table 5.2: Trigger paths used in this analysis and their efficiencies. 


\begin{tabular}{lccc}
\hline Process & Generator & NLO $\sigma \times \mathrm{BR}(\mathrm{pb})$ & $\mathcal{L}\left(\mathrm{pb}^{-1}\right)$ \\
\hline $\mathrm{Z} / \gamma^{*} \rightarrow l l$ & PYTHIA & 17,354 & 7,000 \\
$\mathrm{Z} / \gamma^{*} \rightarrow e e+\gamma$ & BAUR & 14.5 & 204,000 \\
$\mathrm{Z} / \gamma^{*} \rightarrow \mu \mu+\gamma$ & BAUR & 14.5 & 224,000 \\
$\mathrm{Z} / \gamma^{*} \rightarrow e e+\gamma \gamma$ & MADGRAPH & 0.09 & $2,223,000$ \\
$\mathrm{Z} / \gamma^{*} \rightarrow \mu \mu+\gamma \gamma$ & MADGRAPH & 0.09 & $2,178,000$ \\
$\mathrm{~W} \rightarrow e \nu+\gamma$ & BAUR & 30.1 & 99,000 \\
$\mathrm{~W} \rightarrow \mu \nu+\gamma$ & BAUR & 30.1 & 94,000 \\
$\mathrm{~W}+\mathrm{W}$ & PYTHIA & 13.3 & 34,000 \\
$\mathrm{~W}+\mathrm{Z} / \gamma^{*}$ & MADGRAPH & 0.3 & 430,000 \\
$\mathrm{Z} / \gamma^{*}+\mathrm{Z} / \gamma^{*}$ & PYTHIA & 0.3 & 535,000 \\
$t \bar{t}$ & PYTHIA & 6.7 & 143,000 \\
$b \bar{b} / c \bar{c}$ & ISAJET & various & various \\
\hline
\end{tabular}

Table 5.3: Background MC samples used in this analysis. The first column is the background process generated from the proton antiproton collision. The second column is the MC event generator used to simulate the events. The third column is the cross section times branching ratio of the particular process, and the last column is the integrated luminosity of the sample. 


\begin{tabular}{lcc}
\hline Lepton Type & $E_{T}$ or $p_{T}$ Range & Scale Factor \\
\hline TCE & $>5 \mathrm{GeV}$ & 1.0 \\
LCE & $>5 \mathrm{GeV}$ & 1.0 \\
Phoenix & $>15 \mathrm{GeV}$ & 0.949 \\
Plug Stand-Alone & $<15 \mathrm{GeV}$ & $0.925 \times \min \left[\left(0.896+0.0018 \times E_{T}\right), 1\right]$ \\
& & $\times \min \left[\left(0.826+0.0059 \times E_{T}\right), 1\right]$ \\
\hline CMUP & $>20 \mathrm{GeV} / c$ & 0.892 \\
CMUP & $<20 \mathrm{GeV} / c$ & 0.855 \\
CMX & $>20 \mathrm{GeV} / c$ & 0.999 \\
CMX & $<20 \mathrm{GeV} / c$ & 0.906 \\
CMIO & $>10 \mathrm{GeV} / c$ & 1.0 \\
\hline
\end{tabular}

Table 5.4: Lepton identification scale factors $\left(\epsilon_{\text {Data }}^{I D} / \epsilon_{M C}^{I D}\right)$ for each lepton type and $E_{T}$ or $p_{T}$ range. Scale factors are used to correct the MC event predictions due to differences with the data sample. 


\section{CHAPTER 6}

\section{Analysis}

This chapter will describe how the analysis tools presented in the previous chapters are combined to make the final measurement, which consists of counting the number of events in the data signal sample and comparing it with the SM background expectation. Several steps are involved:

- The event selection, specifying exactly which events are accepted and which ones are rejected, must be optimized and finalized.

- Each experimental uncertainty must be determined and combined in order to report an accuracy on the final measurement.

- Tests are run in order to find any faults that may be present in the analysis methods. After all known problems are corrected, then the analysis is considered to be validated.

- Complete the final measurement.

These steps are not independent and often take several iterations before the final measurement is completed. Throughout the process, we attempt to keep the data in the signal sample "blind" in order to prevent knowledge of the events within this sample from biasing the results. 


\subsection{Event Selection}

Choosing event selection criteria involves a balance between maximizing the signal acceptance and minimizing the backgrounds. Ideally, these criteria are chosen based on achieving the best expected result, but this is not always practical since determining the expected result is time consuming and several criteria must be decided before the calculation is even possible. For this thesis, the analysis strategy is to use a minimum of selection cuts to reduce the backgrounds while maintaining a sensitivity not just for $k_{p}$ SUSY events but for other new physics models that produce events with multiple charged leptons in the final state.

To determine the event selection criteria, we start by identifying all leptons within the events that are not tagged as coming from a photon conversion, keeping those events that have at least two leptons. Events that are consistent with cosmic ray muons are removed from consideration. Since the events are required to pass the high- $p_{T}$ lepton triggers, one of the leptons ("trigger lepton") must be either a tight central electron (TCE), or a stubbed muon (either a CMUP or CMX muon), and have $\mathrm{E}_{T}\left(\mathrm{p}_{T}\right)>20 \mathrm{GeV}(\mathrm{GeV} / \mathrm{c})$. The trigger lepton is considered the first lepton in the event while the remaining leptons must have $\mathrm{E}_{T}\left(\mathrm{p}_{T}\right)>8$ and $5 \mathrm{GeV}(\mathrm{GeV} / \mathrm{c})$ for the second and additional leptons, respectively. The plot in Fig. 6.1 shows the expected background contributions of these events. By far, the largest backgrounds are due to Drell-Yan (DY) and those including misidentified jets. In fact the jet background for events with three or more leptons primarily comes from $Z / \gamma^{*}+$ jet events and so the two backgrounds are primarily from the same DY source. To reduce the DY background, events are removed if oppositesign, same-flavor pairs satisfy the following conditions: invariant mass between 76-106 $\mathrm{GeV} / \mathrm{c}^{2}$ (also known as a $Z$ veto), invariant mass below $15 \mathrm{GeV} / \mathrm{c}^{2}$, or $160^{\circ}<\Delta \phi<200^{\circ}$. An event is also rejected if the invariant mass of the two 
highest transverse energy leptons is below $20 \mathrm{GeV} / c^{2}$ to reduce heavy flavor and radiative photon conversion backgrounds. To reduce backgrounds from jets, the leptons are required to be separated from each other by $\Delta \mathrm{R}=\sqrt{(\Delta \eta)^{2}+(\Delta \phi)^{2}}$ $<0.4$. If there are two leptons within $\Delta \mathrm{R}=0.4$, then one of the leptons is removed. In the cases where only one of the leptons is a TCE, CMUP muon, or a CMX muon, then that one is kept, otherwise the lepton with the highest $\mathrm{E}_{T}\left(\mathrm{p}_{T}\right)$ is kept. Since the signal and control samples distinguish between events where the second lepton is an electron or muon, if the second lepton is a CMIO muon it is required to travel through the calorimeter.

\subsection{Signal Samples}

The signal samples define the exact signature used to determine the final result, incorporating the event selecting criteria described in the previous section. The primary signal sample includes all events with four or more leptons and is the

same for both $\lambda_{121} \neq 0$ and $\lambda_{122} \neq 0 \not R_{p}$ SUSY scenarios. In addition, two trilepton samples are defined: one for $\lambda_{121} \neq 0$ in which at least one of the two leading leptons is an electron, and one for $\lambda_{122} \neq 0$ in which at least one of the two leading leptons is a muon. This definition of the trilepton signal samples reduces the background with respect to the signal, since at least two of the four charged leptons must be either electrons or muons for the $\lambda_{121}$ and $\lambda_{122} \ell_{p}$ SUSY scenarios, respectively. Note that these are not exclusive event samples, since events with two electrons and one muon, or two muons and one electron can be included in both trilepton signal samples.

The number of expected events in the signal samples, $N_{\text {exp }}$, is determined for 


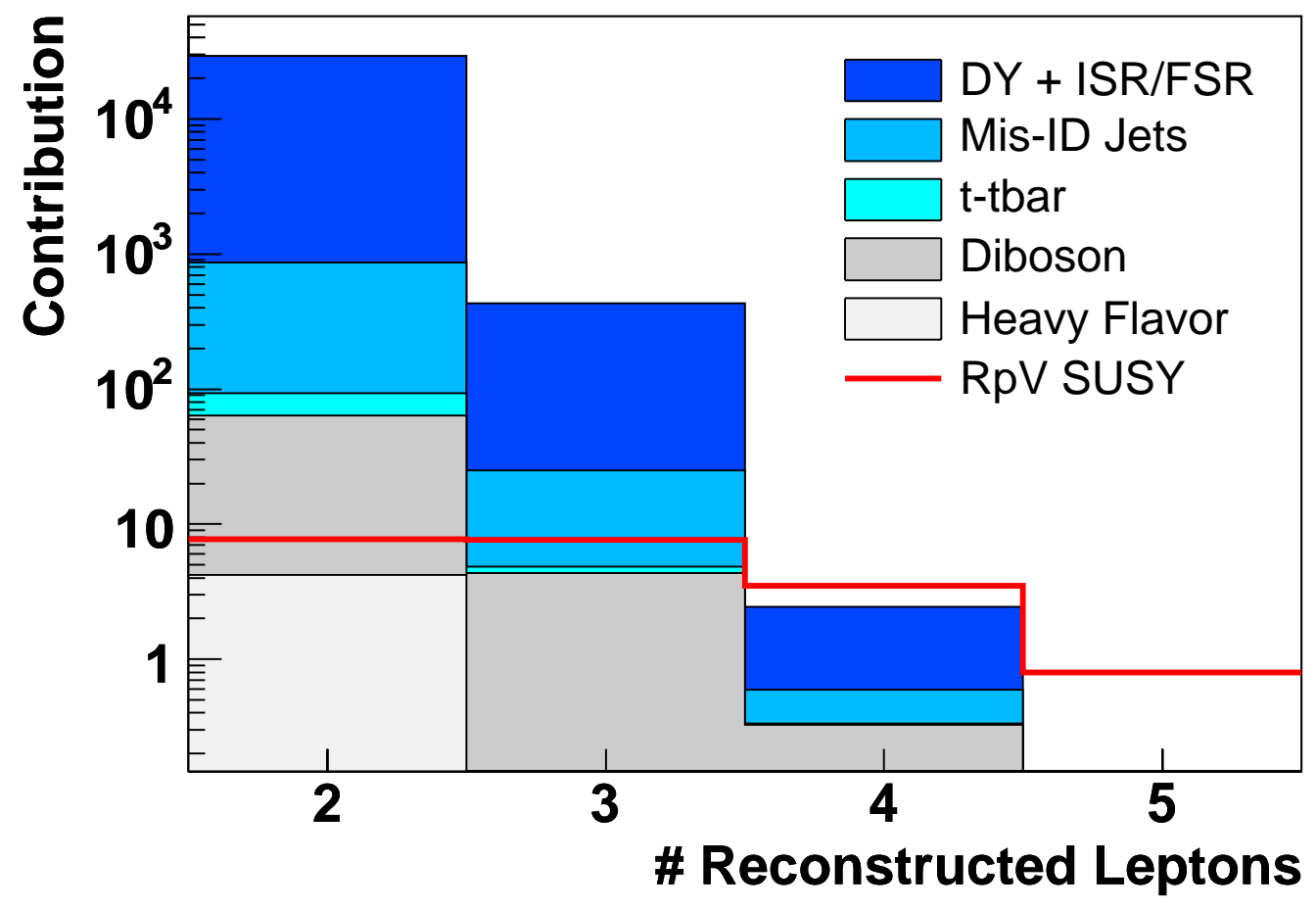

Figure 6.1: Background contributions as a function of the number of reconstructed leptons in each event before the event selection cuts are applied. Each event must have at least one TCE, CMUP, or CMX lepton with $E_{T}\left(p_{T}\right)>20$ $\mathrm{GeV}(\mathrm{GeV} / c)$. The signal $\left(M_{0}=250, M_{1 / 2}=260, \tan \beta=5, A_{0}=0\right.$, and $\left.\mu>0\right)$ and background samples are normalized to $346 \mathrm{pb}^{-1}$. 
the SM background and $\not_{p}$ SUSY by Equation 6.1 :

$$
N_{\text {exp }}=\sigma \times \mathcal{L} \times A
$$

using the corrections described in Sec. 5.4, where $\sigma$ is the appropriate production cross section, $\mathcal{L}$ is the luminosity, and $A$ is the detector acceptance equal to the product of the trigger, reconstruction, and lepton identification efficiencies. $A$ is a measure of how often a certain type of event will be recognized in the detector and is typically $11.0 \pm 0.2 \%$ in the trilepton signal samples and $4.0 \pm 0.1 \%$ in the four or more lepton signal sample for the $\ell_{p}$ SUSY signal. In general, the acceptance increases for larger $\tilde{\chi}_{1}^{0}$ masses. If the observed events within the signal samples significantly exceed the background expectation, then it indicates evidence of previously unidentified particles, possibly supersymmetric particles.

\subsection{Validation}

For any experimental result to be taken seriously, it is necessary to prove that the experimental procedures are done correctly. A simple but powerful way to conduct such a proof is to compare background predictions with observations in control samples, defined as samples in which at least one of the signal event selection requirements fail. For example, an event with only two leptons would not be selected in the signal sample since at least three are required, yet the event could be included inside a control sample. The use of control samples is an effective test since it incorporates the same procedures that are used to find the final result. The main difference is that in the control samples the expected events from the SM backgrounds dominate those expected from $\not_{p}$ SUSY sim-

ulation while in the signal samples the expected $\ell_{p}$ SUSY signal is comparable or much greater than the estimated backgrounds. Since the SM backgrounds are 
well defined, the background prediction should agree with the observation in the control samples, otherwise there is an indication that something is wrong and needs to be corrected.

In this analysis, a total of 26 control samples are used. Control samples are differentiated by lepton types and number of leptons. In most cases the control samples are completely exclusive, meaning that a single event can not appear in two different control samples. The exception is for tight central electrons (TCEs) since it is by definition also a loose central electron (LCEs). The predicted $\mathrm{MC}$ events are compared to the number of observed data events for the $e e, \mu \mu$, and $e \mu$ dilepton control samples in Table 6.1. The background prediction is dominated by DY for the $e e$ and $\mu \mu$ control samples. In the case of $e \mu$ control samples, the biggest background is from $Z / \gamma^{*} \rightarrow \tau \tau$ but there are also significant contributions from misidentified jets, $W W$, and $t \bar{t}$ backgrounds. An excellent agreement between the background prediction and the data is observed for all the dilepton control samples.

The control samples containing events with three or more leptons are listed in Table 6.2. For trilepton samples that fail the $Z$ veto cut, the largest backgrounds are from $Z / \gamma^{*}+\gamma$ and jets misidentified as leptons, as shown in Table 6.3. In the control samples which fail the $\Delta \phi$ cut, the MC prediction is dominated by the $Z / \gamma^{*}+\gamma$ background, as shown in Table 6.4. Since the number of predicted events is small for the trilepton control samples, there is more variation between the background estimation and the data. Still, the overall agreement is good. Besides comparing numbers of events, lepton kinematic distributions are also compared between the data and the MC background prediction. The invariant mass of the first two leptons and the $p_{T}$ of the first lepton are shown in Fig. 6.2 , the $p_{T}$ of the second and third leptons are shown in Fig. $6.3, \Delta \phi$ of the 
first two leptons and the trilepton invariant mass are shown in Fig. 6.4, and the $\mathbb{E}_{T}$ distribution is shown in Fig 6.5. Figs. $6.6-6.8$ demonstrate how well each control sample agrees for the given errors.

Given that there is reasonable agreement between the SM background prediction and the observed events in the control samples, we have confidence that by using the analysis procedures defined in this analysis we can accurately predict the number of expected events in the signal sample.

\subsection{Uncertainties}

There are two major types of uncertainties that factor into this search: statistical and systematic.

\subsubsection{Statistical Uncertainty}

Statistical uncertainties deal with the element of randomness for a particular measurement. Suppose we want to measure the probability of getting heads by flipping a coin. If we flip the coin only once it will come up either heads or tails corresponding to a probability of $0 \%$ or $100 \%$. By repeating this procedure over and over again, we would observe that the probability converges at $50 \%$

reflecting the "true" probability. By increasing the number of measurements, the uncertainty is reduced but can never be completely eliminated. In this analysis, statistical uncertainties are involved in the background predictions. For each background, there is an uncertainty on the probability that an event will pass the selection criteria. Each event may either pass or fail, just like with the coin toss, although the true probability for this to occur may be very different from $50 \%$. Since the background samples are large (millions of events), we expect 


\begin{tabular}{lcll}
\hline Control Sample & Cut Failed & MC Prediction & Data \\
\hline$e e:$ TCE Z & $Z$ Veto & $5682 \pm 612$ & 5703 \\
$e e: 2$ TCE No Z & - & $1117 \pm 119$ & 1041 \\
$e e:$ TCE + LCE Z & $Z$ Veto & $7318 \pm 800$ & 7483 \\
$e e:$ TCE + LCE No Z & - & $1426 \pm 154$ & 1416 \\
$e e:$ TCE + Plug Z & $Z$ Veto & $6630 \pm 735$ & 6536 \\
$e e:$ TCE + Plug No Z & - & $716 \pm 80$ & 709 \\
\hline$\mu \mu:$ TM + CMUP Z & $Z$ Veto & $2388 \pm 244$ & 2425 \\
$\mu \mu:$ TM + CMUP No Z & - & $453 \pm 49$ & 518 \\
$\mu \mu:$ TM + CMX Z & $Z$ Veto & $1809 \pm 183$ & 1845 \\
$\mu \mu:$ TM + CMX No Z & - & $306 \pm 33$ & 325 \\
$\mu \mu:$ TM + CMIO Z & $Z$ Veto & $3277 \pm 400$ & 3229 \\
$\mu \mu:$ TM + CMIO No Z & - & $505 \pm 61$ & 496 \\
\hline$e \mu:$ TM + TCE & - & $72.7 \pm 7.3$ & 76 \\
$e \mu:$ TM + LCE & - & $137.2 \pm 19.9$ & 152 \\
$e \mu:$ TM + PLUG & - & $51.6 \pm 7.2$ & 51 \\
$e \mu:$ TCE + CMUP & - & $42.1 \pm 4.7$ & 45 \\
$e \mu:$ TCE + CMX & - & $24.8 \pm 2.8$ & 25 \\
$e \mu:$ TCE + CMIO & - & $50.7 \pm 7.3$ & 42 \\
\hline
\end{tabular}

Table 6.1: List of the dilepton control samples used in this analysis. control samples are split up by lepton type and number of leptons. TM = trigger muon, either CMUP or CMX. Errors include systematic uncertainties and statistical uncertainty based on the number of generated MC events. 


\begin{tabular}{|c|c|c|c|}
\hline Control Sample & Cut Failed & MC Prediction & Data \\
\hline eel & Z Veto & $8.8 \pm 1.9$ & 12 \\
\hline$\mu \mu \ell$ & Z Veto & $5.1 \pm 1.2$ & 2 \\
\hline$e \mu \ell$ & Z Veto & $0.55 \pm 0.04$ & 0 \\
\hline$\ell \ell \ell$ & Z Veto & $14.4 \pm 2.9$ & 14 \\
\hline eel & $\Delta \phi$ & $2.1 \pm 0.3$ & 2 \\
\hline$\mu \mu \ell$ & $\Delta \phi$ & $1.2 \pm 0.2$ & 4 \\
\hline$e \mu \ell$ & $\Delta \phi$ & $0.35 \pm 0.04$ & 0 \\
\hline$\ell \ell \ell$ & $\Delta \phi$ & $3.7 \pm 0.3$ & 6 \\
\hline 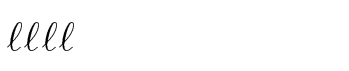 & Z Veto & $0.15 \pm 0.02$ & 0 \\
\hline 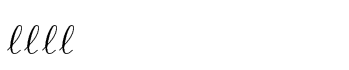 & $\Delta \phi$ & $0.006 \pm 0.003$ & 0 \\
\hline
\end{tabular}

Table 6.2: List of the $\geq 3$-lepton control samples used in this analysis. Control samples are split up by lepton type and number of leptons. The variable $\ell$ refers to either an electron or muon. Errors include systematic uncertainties and statistical uncertainty based on the number of generated MC events. 


\begin{tabular}{|c|c|c|c|}
\hline \multicolumn{4}{|c|}{ Trilepton Z Control Sample } \\
\hline & eel & $\mu \mu \ell$ & $e \mu \ell$ \\
\hline$Z / \gamma^{*} \rightarrow e e+\gamma$ & $4.6 \pm 0.8$ & 0 & 0 \\
\hline$Z / \gamma^{*} \rightarrow \mu \mu+\gamma$ & 0 & $2.6 \pm 0.5$ & $0.15 \pm 0.02$ \\
\hline$W+Z / \gamma^{*}$ & $0.80 \pm 0.06$ & $0.35 \pm 0.03$ & $0.36 \pm 0.03$ \\
\hline Total mis-ID jets & $3.5 \pm 1.7$ & $2.1 \pm 1.1$ & $0.04 \pm 0.02$ \\
\hline Total Background & $8.8 \pm 1.9$ & $5.1 \pm 1.2$ & $0.55 \pm 0.04$ \\
\hline Data & 12 & 2 & 0 \\
\hline
\end{tabular}

Table 6.3: Numbers of predicted and observed trilepton events containing two opposite-sign same-flavor leptons that form an invariant mass between 76 and 106 $\mathrm{GeV} / \mathrm{c}^{2}$. The control sample is broken down by lepton channel and background type.

\begin{tabular}{|c|c|c|c|}
\hline \multicolumn{4}{|c|}{ Trilepton $\Delta \phi$ Control Sample } \\
\hline & $e e \ell$ & $\mu \mu \ell$ & $e \mu \ell$ \\
\hline$Z / \gamma^{*} \rightarrow e e+\gamma$ & $1.9 \pm 0.3$ & 0 & 0 \\
\hline$Z / \gamma^{*} \rightarrow \mu \mu+\gamma$ & 0 & $1.0 \pm 0.1$ & $0.34 \pm 0.05$ \\
\hline$W+Z / \gamma^{*}$ & $0.030 \pm 0.005$ & $0.011 \pm 0.002$ & $0.008 \pm 0.002$ \\
\hline Total mis-ID jets & $0.21 \pm 0.11$ & $0.14 \pm 0.07$ & 0 \\
\hline Total Background & $2.1 \pm 0.3$ & $1.2 \pm 0.2$ & $0.35 \pm 0.04$ \\
\hline Data & 2 & 4 & 0 \\
\hline
\end{tabular}

Table 6.4: Numbers of predicted and observed trilepton events containing two opposite-sign same-flavor leptons that have $160^{\circ}<\Delta \phi<200^{\circ}$. The control sample is broken down by lepton channel and background type. 
this number of passing events to obey Poisson statistics [65]. Therefore, the statistical uncertainty on the background prediction from an individual process is proportional to $\sqrt{N}$ for $N \gtrsim 10$, where $N$ is the number of events that pass the selection criteria.

In this analysis, statistics makes up the largest uncertainty for the background prediction in the signal samples. In particular, the largest contribution is due to the $Z / \gamma^{*}+\gamma$ background. As described in Sec. 5.3.3, for a photon to be misidentified as a prompt electron, it must convert into an electron-positron pair where one of the tracks is not reconstructed. Unfortunately, these events can not be simulated directly. In the $\mathrm{MC}$ event generators, we are limited to producing events with photons, which may or may not convert. Therefore, in order to make a background prediction for trilepton events where a photon conversion is misidentified as an electron, a large sample of $Z / \gamma^{*}+\gamma$ events must be generated.

The size of these samples, which is $\sim 3$ million events for both $Z / \gamma^{*} \rightarrow e e+\gamma$ and $Z / \gamma^{*} \rightarrow \mu \mu+\gamma$ samples, makes it impractical to considerably lower the statistical uncertainty by generating additional events.

\subsubsection{Systematic Uncertainty}

Systematic uncertainties reflect the limitations on our knowledge of all the individual pieces that go into the final result. These types of uncertainties are much harder to estimate since it is difficult to quantify the unknown. For the most part, the process of assigning systematic uncertainties is subjective because it relies on the experimenter's prior knowledge. In order to prevent systematic uncertainties from varying wildly between different experiments and to reduce the differences based on subjectivity, particle physicists attempt to define standard procedures for identifying and determining these uncertainties. Often these 
procedures involve varying analysis methods and input values, and taking the variation in the result as the uncertainty. For example, in measuring the length of book using a ruler, one could change rulers to see if there is any variation in length. Since the book's length is independent of the ruler used to measure it, any variation reflects an uncertainty in the accuracy of the ruler and therefore on the measurement itself.

In this analysis, systematic uncertainties are determined for the following categories: jets misidentified as leptons, lepton identification requirements, photon conversion identification, luminosity measurement, choice of parton distribution functions (PDFs), cross section measurement for both $\not_{p}$ SUSY and backgrounds, QCD initial state radiation (ISR), and QCD final state radiation (FSR). The uncertainty on the trigger efficiencies, listed in Table 5.2, are known to better than $0.5 \%$ and are not considered.

The uncertainty associated with the probability for a jet to be misidentified as a lepton is determined to be $50 \%$ of the misidentification rate, as described in Section 4.5. This uncertainty is applied to the predicted number of jets misidentified as leptons. For example, if 1.0 events are predicted, then the uncertainty is \pm 0.5 events.

The process for measuring lepton identification efficiencies $\left(\epsilon_{I D}\right)$ was described in Section 4.4. Uncertainties on this measurement come from how well the lepton ID cuts are understood and how well the lepton sample is understood, where the lepton sample refers to the denominator of the $\epsilon_{I D}$ measurement. Since the number of events in the signal sample is based on the detector simulation, another source of uncertainty is derived from how well the simulation is understood. Uncertainties on the lepton ID efficiency are determined by first modifying one of the criteria in selecting the lepton sample or by modifying one of the lepton identifica- 
tion cuts, and second measuring the resulting change in efficiency. For example, the lepton sample can be changed by using a different method of background subtraction. If the new background subtraction method results in a different $\epsilon_{I D}$, then the variation between the two values of $\epsilon_{I D}$ can be used to determine a systematic uncertainty on the efficiency. The uncertainties associated with lepton identification are listed in Table 6.5 for each lepton type and $\mathrm{p}_{T}$ range. Lepton identification uncertainties, which are assumed to be correlated, are applied per lepton in each event. Therefore, for events with three leptons, the total lepton ID uncertainty is obtained by adding the three individual uncertainties.

Photon conversion identification was discussed in Section 4.7. Uncertainties related to identifying photon conversions include how well the photon conversion sample is understood, how well the photon conversion selection is understood, and how well the simulation models the detector. A complete description of this uncertainty is described in Appendix B. The total uncertainty is determined to be $28.8 \%$ per photon conversion in the simulation which is misidentified as a single electron.

There is a $6 \%$ uncertainty on the luminosity measurement due to how well the CLC detector is understood and how well the proton antiproton inelastic cross section is understood at a center of mass energy of $1.96 \mathrm{TeV}$.

PDFs, which describe structure of hadrons (Sec. 2.6), are obtained by parameterizing experimental data that is sensitive to the parton structure. Various sets of PDFs, have been compiled by the CTEQ [66] and MRST [67] collaborations. In this analysis the CTEQ6M PDFs are used, consisting of the best fit $\left(S_{0}\right)$ and 20 eigenvector basis sets in the plus $\left(S_{i}^{+}\right)$and minus $\left(S_{i}^{-}\right)$directions along each eigenvector. To calculate the uncertainty on the acceptance $(\Delta A)$ due to the 
PDFs, the following formula provided by the CTEQ collaboration is used:

$$
\Delta A=\frac{1}{2}\left(\sum_{i=1}^{20}\left[A\left(S_{i}^{+}\right)-A\left(S_{i}^{-}\right)\right]^{2}\right)^{1 / 2}
$$

where $\mathrm{A}\left(S_{i}^{ \pm}\right)$is the acceptance based on the plus and minus PDF set $i$. The systematic uncertainty based on the PDFs is determined to be $0.3 \%$ for the $\not k_{p}$ SUSY simulation and $2.0 \%$ for each background in the signal sample.

The $q \bar{q} \rightarrow \tilde{\chi}_{2}^{0} \tilde{\chi}_{1}^{ \pm}$and $q \bar{q}^{\prime} \rightarrow \tilde{\chi}_{1}^{ \pm} \tilde{\chi}_{1}^{\mp}$ cross section calculations involve perturbative QCD, which only holds for small values of the strong coupling constant $\left(\alpha_{s}\right)$. Due to asymptotic freedom, small values of $\alpha_{s}$ correspond to large values of the energy scale $\left(\mathrm{Q}^{2}\right)$. Therefore, the PROSPINO2 program only calculates the cross section down to a cutoff value of $\mathrm{Q}^{2}$ chosen to be the average value of the $\tilde{\chi}_{1}^{ \pm}$and $\tilde{\chi}_{2}^{0}$ masses. This cutoff value is somewhat arbitrary and so we assign a systematic uncertainty due to changes in the cross section when the cutoff value is doubled and halved. The $\mathrm{Q}^{2}$ dependence is done for the backgrounds using the same method. In addition, changing the PDFs may also result in a change in cross section. This is a different effect from the previous uncertainty, where it was only determined how the PDF affected the acceptance and not the cross section. The uncertainties on the cross sections are listed in Table 6.6.

ISR and FSR systematic uncertainties are determined with PYTHIA using a parton shower approach [44] to determine the amount and nature of QCD radiation. For ISR and FSR, parameters controlling the parton shower process within the $\mathrm{MC}$ event generator are tuned such that the $p_{T}$ spectrum in DY events match the data. By changing the parameters listed in Table 6.7, events are produced with more or less ISR or FSR, which changes the kinematic distributions of the jets in the events. Systematic uncertainties are assigned for ISR and FSR by taking half the percent difference in acceptance between samples with more or less ISR and FSR. For the $\not_{p}$ SUSY simulation the ISR systematic uncertainty 


\begin{tabular}{llc}
\hline Lepton Type & $\mathrm{E}_{T}$ or $\mathrm{p}_{T}$ Range & Uncertainty (\%) \\
\hline CMUP Muon & $\mathrm{p}_{T}>20 \mathrm{GeV} / \mathrm{c}$ & 1.1 \\
CMUP Muon & $\mathrm{p}_{T}<20 \mathrm{GeV} / \mathrm{c}$ & 6.1 \\
CMX Muon & $\mathrm{p}_{T}>20 \mathrm{GeV} / \mathrm{c}$ & 0.7 \\
CMX Muon & $\mathrm{p}_{T}<20 \mathrm{GeV} / \mathrm{c}$ & 6.1 \\
CMIO Muon & $\mathrm{All}_{T}$ & 6.0 \\
TCE & $\mathrm{E}_{T}>8 \mathrm{GeV}$ & 2.0 \\
LCE & $\mathrm{E}_{T}>8 \mathrm{GeV}$ & 4.0 \\
TCE or LCE & $\mathrm{E}_{T}<8 \mathrm{GeV}$ & 15 \\
Plug Electron & $\mathrm{E}_{T}>15 \mathrm{GeV}$ & 2.8 \\
Plug Electron & $\mathrm{E}_{T}<15 \mathrm{GeV}$ & 11.0 \\
\hline
\end{tabular}

Table 6.5: Systematic uncertainties associated with lepton identification for each lepton type and $\mathrm{p}_{T}$ range.

is determined to be $1.5 \%$ independent of the mSUGRA parameters. For the background, an ISR systematic uncertainty of $4 \%$ is used based on different measurements with similar backgrounds $[61,68]$. The FSR systematic uncertainty is negligible for both the signal and background samples.

The individual uncertainties described above are treated as independent quantities and are combined by adding each one in quadrature. In the $\lambda_{121}\left(\lambda_{122}\right)$ trilepton signal samples, the total systematic uncertainty is $11.7 \%$ (12.0\%) for the $\not R_{p}$ SUSY MC and $15.7 \%(17.0 \%)$ for the background. In the $\geq 4$-lepton signal sample, the total systematic uncertainty is either $14.3 \%\left(\lambda_{121}\right)$ or $15.0 \%$ $\left(\lambda_{122}\right)$ for the $\not_{p}$ SUSY MC and $42.6 \%$ for the background. The individual and combined systematic uncertainties are shown in Table 6.8 for each of the signal samples. 


\begin{tabular}{lc}
\hline Process & Uncertainty (\%) \\
\hline DY & 8 \\
Diboson & 5 \\
$t \bar{t}$ & 10 \\
$k_{p}$ SUSY & 6.1 \\
\hline
\end{tabular}

Table 6.6: Systematic uncertainties associated with the theoretical cross section for each process. The numbers include uncertainties from both $Q^{2}$ variation and variation between $\mathrm{PDF}$ sets.

\begin{tabular}{lc}
\hline ISR/FSR & Pythia Parameters \\
\hline Default ISR & $\operatorname{PARP}(61)=0.146 \mathrm{GeV}, \operatorname{PARP}(64)=1.0$ \\
More ISR & $\operatorname{PARP}(61)=0.292 \mathrm{GeV}, \operatorname{PARP}(64)=0.5$ \\
Less ISR & $\operatorname{PARP}(61)=0.073 \mathrm{GeV}, \operatorname{PARP}(64)=2.0$ \\
\hline Default FSR & $\operatorname{PARP}(72)=0.146 \mathrm{GeV}, \operatorname{PARP}(71)=4.0$ \\
More FSR & $\operatorname{PARP}(72)=0.292 \mathrm{GeV}, \operatorname{PARP}(71)=8.0$ \\
Less FSR & $\operatorname{PARP}(72)=0.073 \mathrm{GeV}, \operatorname{PARP}(71)=2.0$ \\
\hline
\end{tabular}

Table 6.7: PYTHIA parameters used in determining the ISR and FSR systematic uncertainties for the $\not R_{p}$ SUSY signal samples. 

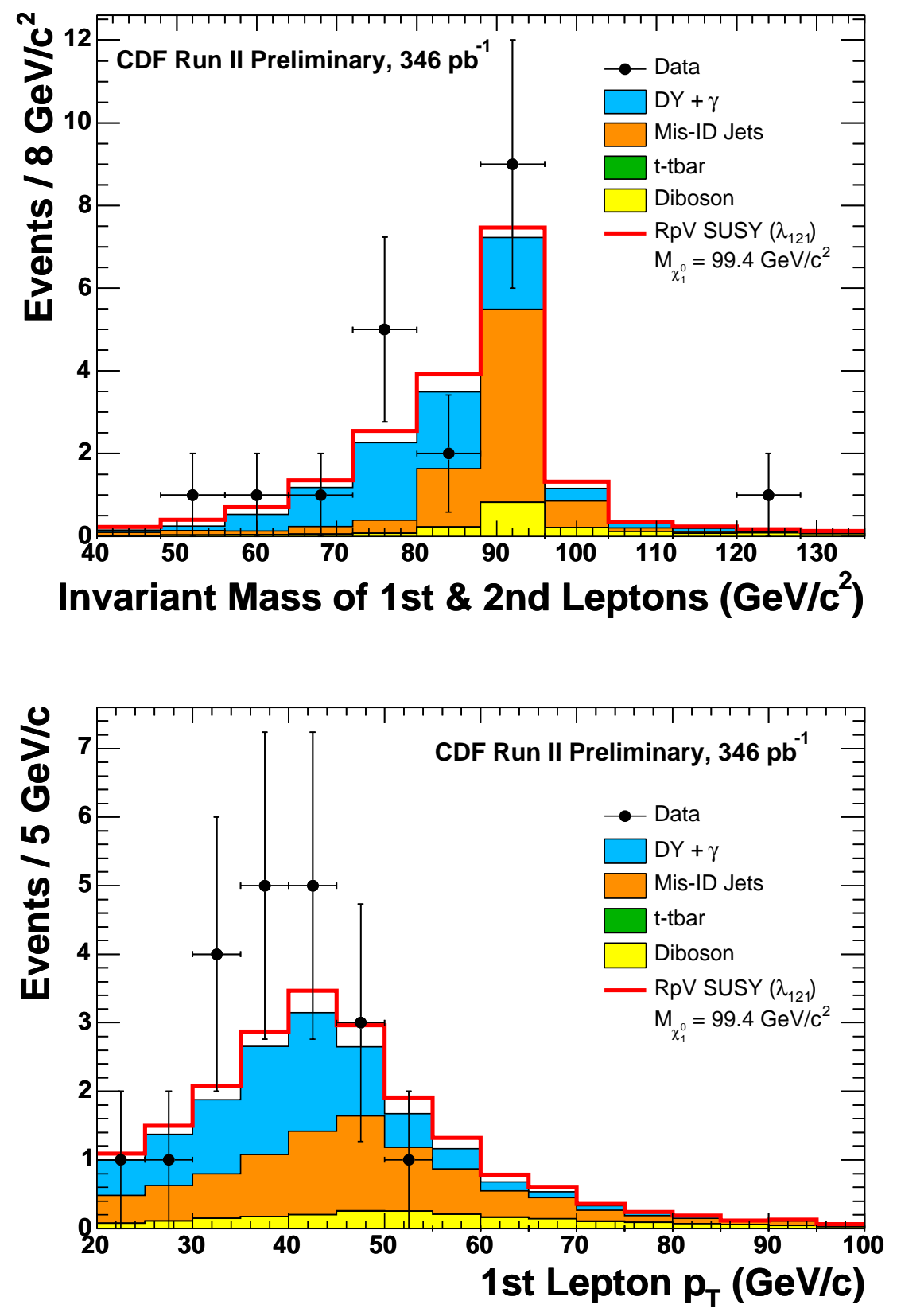

Figure 6.2: Invariant mass, and $\mathrm{p}_{T}$ distributions for the events within the trilepton control samples. The $\not R_{p}$ SUSY signal $\left(M_{0}=250, M_{1 / 2}=260, \tan \beta=5, A_{0}=0\right.$, and $\mu>0)$ is stacked on top of the backgrounds. 

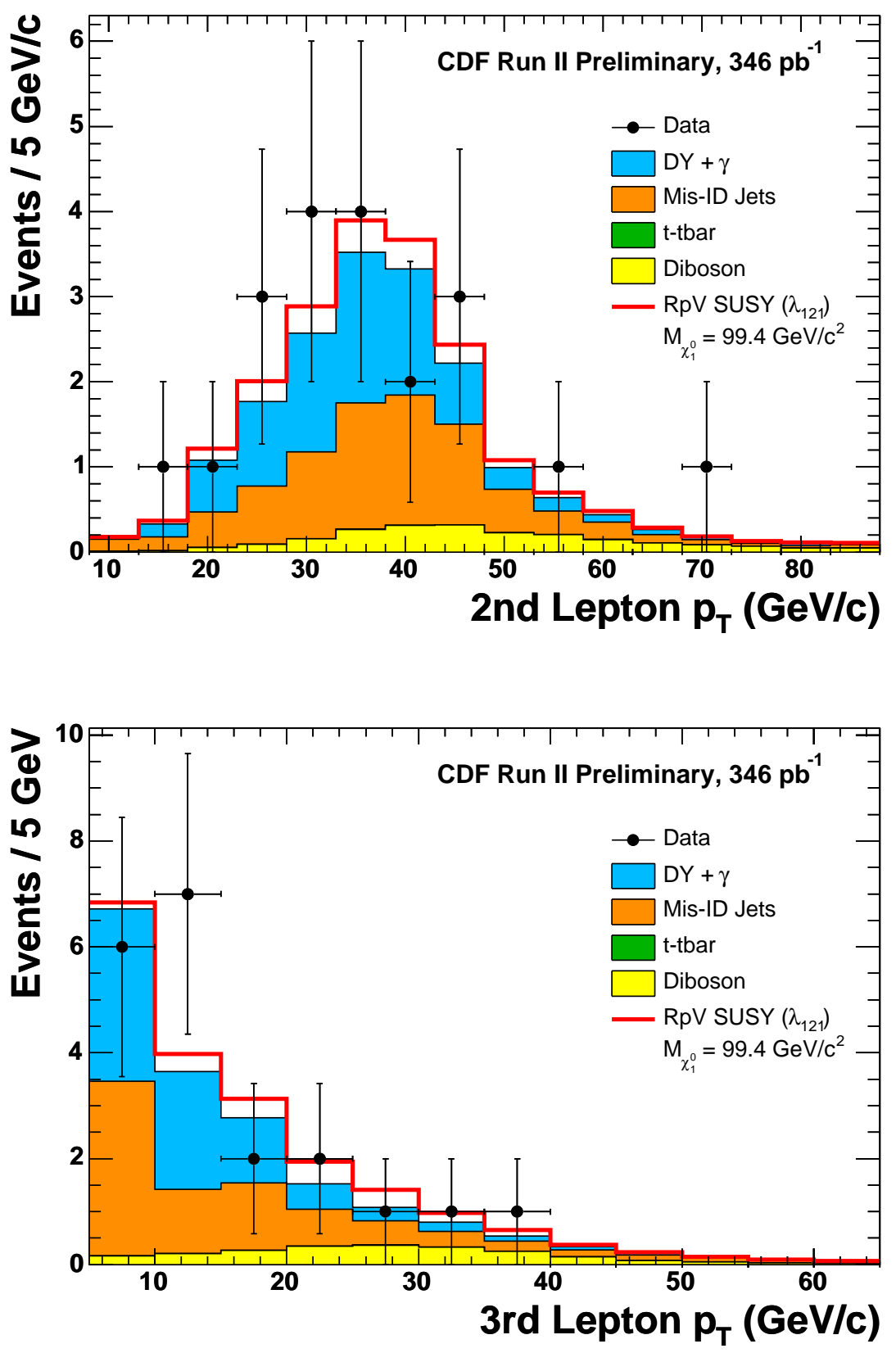

Figure 6.3: 2nd and 3rd lepton $\mathrm{p}_{T}$ distributions for the events within the trilepton control samples. The $\not R_{p}$ SUSY signal $\left(M_{0}=250, M_{1 / 2}=260, \tan \beta=5, A_{0}=0\right.$, and $\mu>0)$ is stacked on top of the backgrounds. 

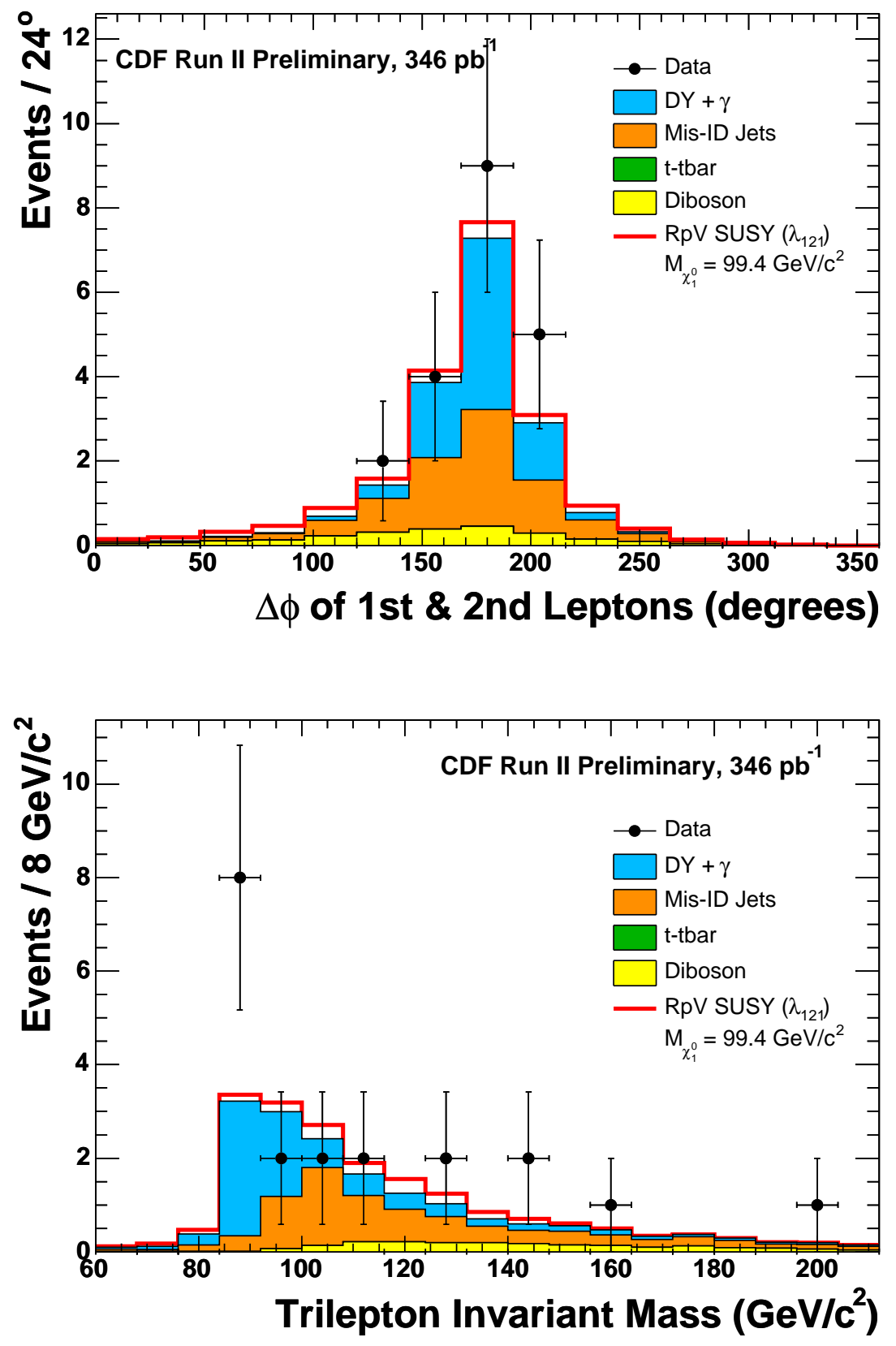

Figure 6.4: $\Delta \phi$ (top plot) and trilepton invariant mass (bottom plot) distributions for the events within the trilepton control samples. The $\not_{p}$ SUSY signal $\left(M_{0}=250, M_{1 / 2}=260, \tan \beta=5, A_{0}=0\right.$, and $\left.\mu>0\right)$ is stacked on top of the backgrounds. 


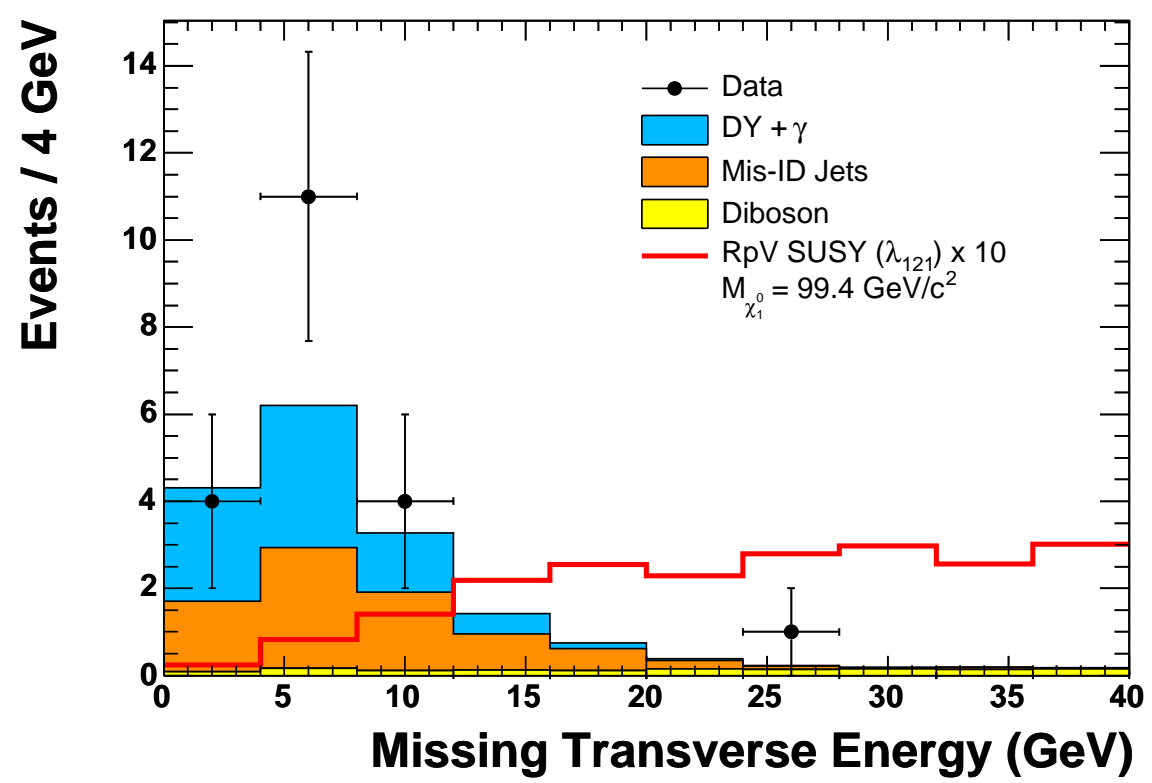

Figure 6.5: Missing transverse energy distribution for the events within the trilepton control samples. The $R_{p}$ SUSY signal $\left(M_{0}=250, M_{1 / 2}=260, \tan \beta=5\right.$, $A_{0}=0$, and $\mu>0$ ) is multiplied by a factor of 10 for easier visibility and plotted independently of the backgrounds. 


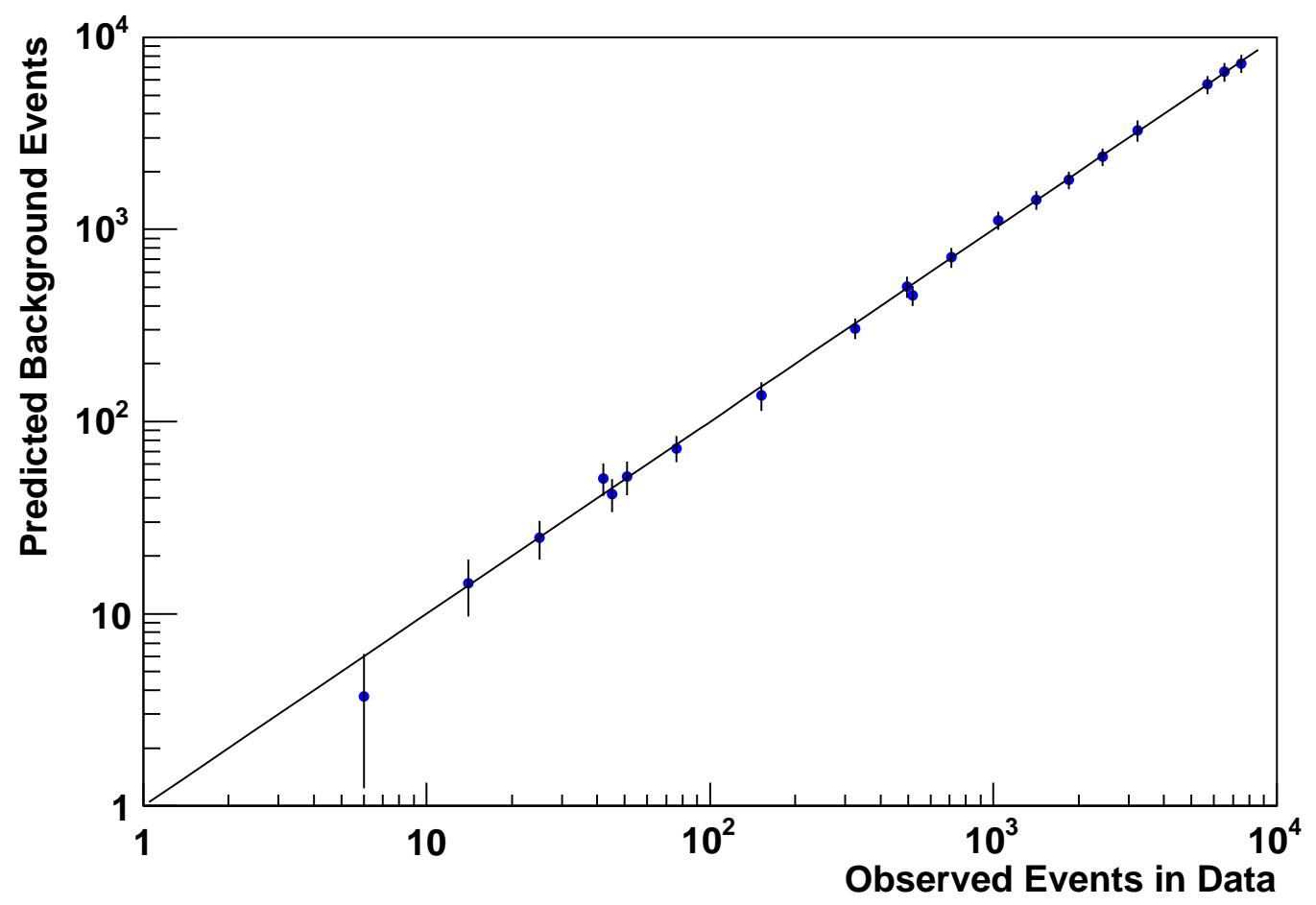

Figure 6.6: Scatter plot of the predicted background events ( $y$ axis) versus the number of observed events in data ( $x$ axis) for each control sample. The points correspond to the control samples while the vertical error bars corresponds to the combined uncertainty on the data and MC. Due to lack of statistics, the trilepton control samples are not broken down by lepton family. The line is a visual guide signifying perfect agreement between data and MC. 

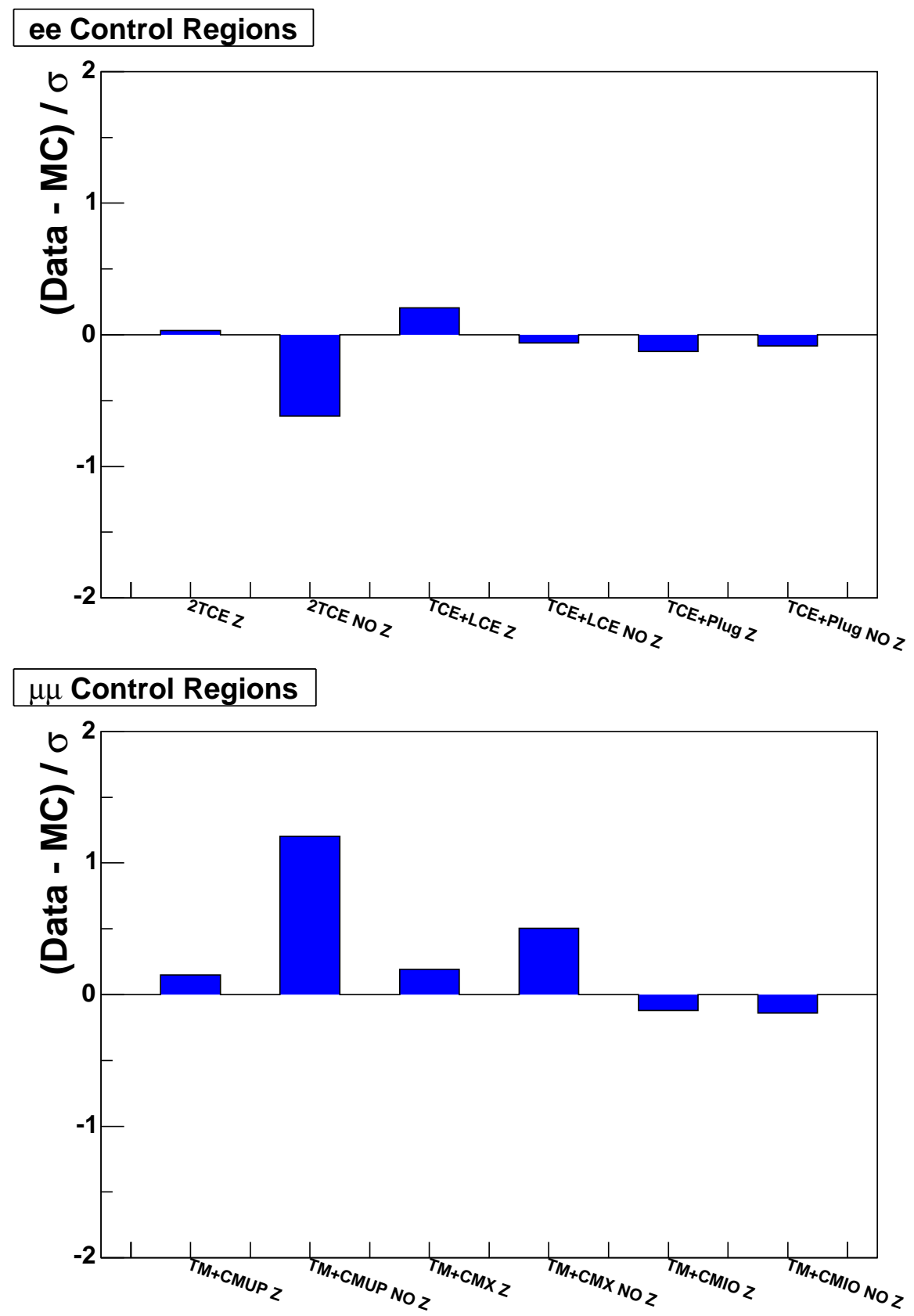

Figure 6.7: Agreement between data and simulation in standard deviations per control sample. The statistical uncertainty in data and the MC uncertainty are added in quadrature. Dielectron control samples (top) and dimuon control samples (bottom). 

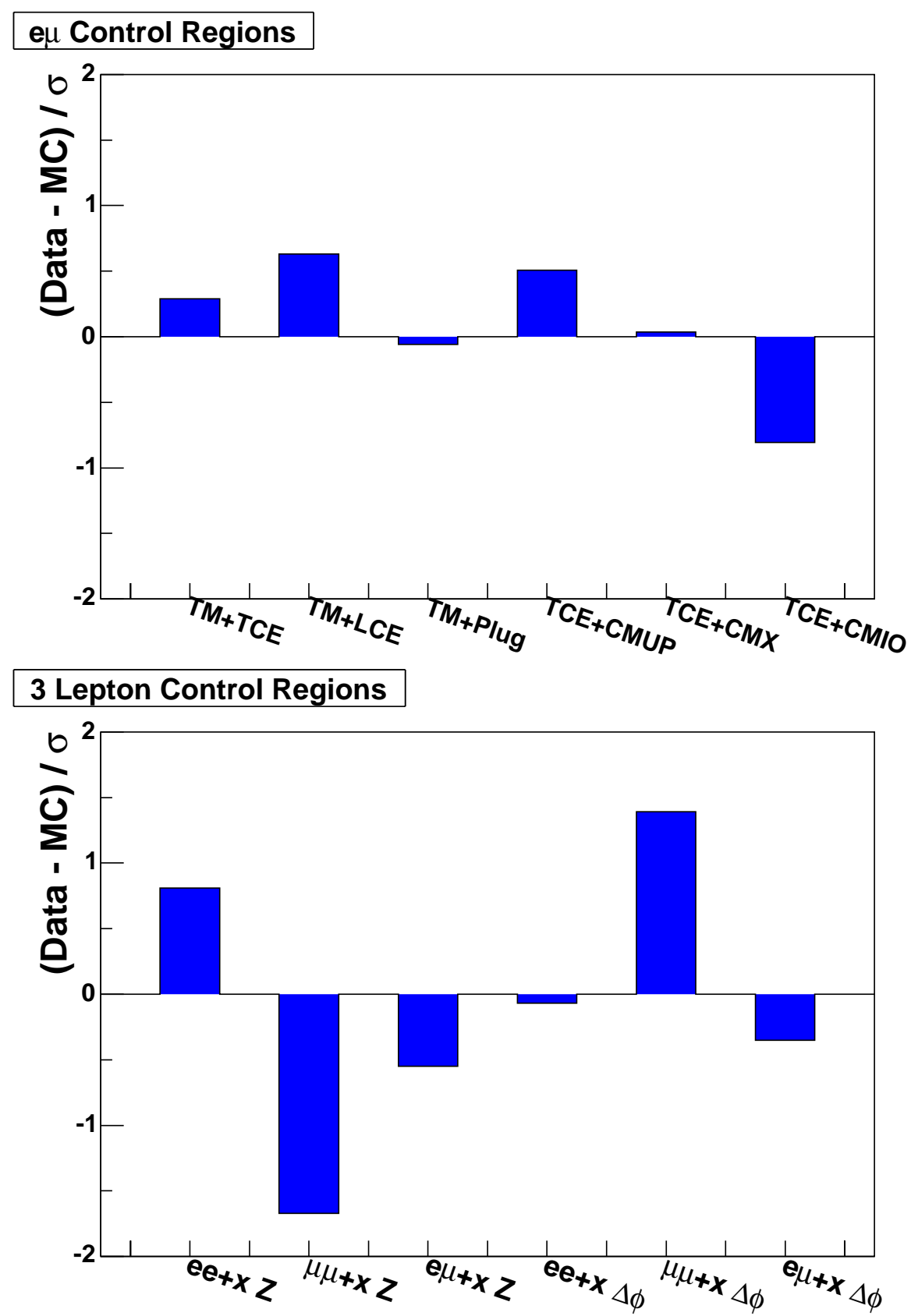

Figure 6.8: Agreement between data and simulation in standard deviations per control sample. The statistical uncertainty in data and the MC uncertainty are added in quadrature. Dilepton - e $\mu$ control samples (top) and trilepton control samples (bottom). TM = trigger muon, either CMUP or CMX. 


\begin{tabular}{lcccccccc}
\hline \multicolumn{7}{c}{ Systematic Uncertainty (\%) } \\
\hline & 3 lep $\lambda_{121}$ & & 3 lep & $\lambda_{122}$ & $\geq 4$ lep $\lambda_{121}$ & $\geq 4$ lep $\lambda_{122}$ \\
\hline & Bg. & SUSY & Bg. & SUSY & Bg. & SUSY & Bg. & SUSY \\
\hline Luminosity & 6.0 & 6.0 & 6.0 & 6.0 & 6.0 & 6.0 & 6.0 & 6.0 \\
PDF & 2.0 & 0.3 & 2.0 & 0.3 & 2.0 & 0.3 & 2.0 & 0.3 \\
ISR & 4.0 & 1.5 & 4.0 & 1.5 & 4.0 & 1.5 & 4.0 & 1.5 \\
Jet mis-ID & 11.5 & - & 12.8 & - & 41.4 & - & 41.4 & - \\
$\gamma \rightarrow$ ee & 2.6 & - & 3.3 & - & 0 & - & 0 & - \\
Lepton ID & 4.7 & 8.0 & 5.8 & 8.4 & 5.9 & 11.4 & 5.9 & 12.3 \\
Cross Section & 5.5 & 6.1 & 5.1 & 6.1 & 2.3 & 6.1 & 2.3 & 6.1 \\
\hline Total & $\mathbf{1 5 . 7}$ & $\mathbf{1 1 . 7}$ & $\mathbf{1 7 . 0}$ & $\mathbf{1 2 . 0}$ & $\mathbf{4 2 . 6}$ & $\mathbf{1 4 . 3}$ & $\mathbf{4 2 . 6}$ & $\mathbf{1 5 . 0}$ \\
\hline
\end{tabular}

Table 6.8: Contribution from each source of systematic uncertainty to the total uncertainty for both $\not R_{p}$ SUSY and background in each signal sample. 


\section{CHAPTER 7}

\section{Results}

In Section 6.2, the signal samples were defined such that the number of expected $R_{p}$ SUSY events for a particular choice of parameters are at least comparable to the number of expected SM background events. To prevent any personal bias from influencing the final result, the process of comparing the data to the background expectation in the signal samples should be the last step of the analysis. In actuality, this plan was not realized since the event selection was slightly changed to the one defined in Section 6.1 after looking at the trilepton data inside the signal sample, although care was still taken in order to prevent biasing the results. The full explanation for changing the event selection can be found in Appendix A. In this section, only the results corresponding to the final event selection will be shown.

In the trilepton signal sample, there are a total of 6 events, 5 with an expected SM background of $3.1 \pm 0.7$ (stat.) \pm 0.4 (syst.) events in the $\lambda_{121} \neq 0$

scenario, and one event with an expected SM background of $1.9 \pm 1.0$ (stat.) \pm 0.3 (syst.) events in the $\lambda_{122} \neq 0$ scenario, as shown in Table 7.1. In the four lepton signal sample, no events were observed with an expected background of $0.008 \pm 0.003$ (stat.) \pm 0.003 (syst.) events, as shown in Table 7.2. These findings are interpreted as being consistent with the hypothesis of SM background and no $\not_{p}$ SUSY signal. While there is an excess of data events in the $\lambda_{121}$ trilepton signal sample, there is a $20 \%$ chance that the expected background could 
fluctuate to the observed number of events or more. A tell-tale sign of physics beyond the SM would be an excess of two or more events in the four or more lepton signal sample where the background prediction is much smaller.

Of the six CDF data events within the trilepton signal samples, four are eee events, one is an ee $\mu$ event, and one is a $\mu \mu e$ event. Each event is in either the $\lambda_{121}$ or $\lambda_{122}$ signal samples but not both. The first three events are described in Table 7.3 and the last three events are described in Table 7.4. Four of these events (3 eee and $1 \mu \mu e$ ) are consistent with the $Z / \gamma^{*}+\gamma$ background since the combined invariant mass of all the leptons is close to the $Z$ mass (Fig. 7.1). This suggests that all three final state particles originated from the $Z$ boson. If the three leptons originated from two separate decays of the neutralino as in Fig. 2.11, then we would expect the trilepton invariant mass to have a much broader distribution. One of these events, which contains three electrons, is displayed in Figure 7.2. In this event the first two electrons most likely originate from a $Z$ boson since they are opposite sign and form an invariant mass of $70 \mathrm{GeV} / c^{2}$ just below the cut of $76 \mathrm{GeV} / c^{2}$. The third electron, most likely comes from a photon conversion, where the photon was radiated from the second electron. The two trilepton events that do not seem to be due to $Z / \gamma^{*}+\gamma$ production may include jets misidentified as charged leptons, but this is unclear.

The $\mathbb{E}_{T}$ distribution in Fig. 7.3 is another indication that the events in the trilepton signal sample are due to DY events. For five of the events, $\mathbb{E}_{T}$ is lower than $13 \mathrm{GeV}$ while the other event contains $\boldsymbol{E}_{T}$ of $\sim 23 \mathrm{GeV}$ which is consistent with the SM background prediction. Additional distributions include the invariant mass of the first two leptons and the $p_{T}$ of the first lepton in Fig. 7.4, the $p_{T}$ of the second and third leptons in Fig. 7.5, and the $\Delta \phi$ of the first two leptons and the sum of the lepton $p_{T}$ 's in Fig. 7.6. 
Although no significant indication of physics beyond the SM was observed, this does not entirely rule out $\ell_{p}$ SUSY. For increasing superpartner masses, the $k_{p}$ SUSY cross section decreases. Therefore this analysis is only sensitive to superpartner masses below a certain value. The mass limits are calculated at the $95 \%$ confidence level (CL) by first obtaining the cross section limits. To determine the upper limit on the $\not_{p}$ SUSY cross section, the number of observed events, number of expected background events, $R_{p}$ SUSY acceptance, and all uncertainties for each signal sample are entered into a Bayesian limit program. The program is similar to [69], except that it incorporates signal and background predictions and observed results from multiple samples. The limit calculation treats uncertainties as nuisance parameters which are allowed to be correlated between the different samples. This procedure is done for each $k_{p}$ SUSY point combining the trilepton and four or more lepton signal samples, shown for $3 \times$ $10^{-3} \lesssim \lambda_{121} \lesssim 0.14$ in Table 7.5 and for $3 \times 10^{-3} \lesssim \lambda_{122} \lesssim 0.14$ in Table 7.6. It is interesting to note that the cross section limit is improved by splitting up the signal sample into two separate ones by $12 \%$ and $4 \%$ in the $\lambda_{121}$ and $\lambda_{122}$ signal samples, respectively, as shown in Table 7.7. The cross section limits for each $h_{p}$ SUSY point are then combined to calculate the lower limit on the $\tilde{\chi}_{1}^{0}$ and $\tilde{\chi}_{1}^{ \pm}$particles, by determining where the observed cross section limit intersects the $\not k_{p}$ SUSY cross section. Mass limit plots are shown in Figure 7.7 for $\lambda_{121}, \mu>$ 0 and for all other investigated scenarios in Figs. 7.8 - 7.10. Both the expected and observed mass limits are presented in Figure 7.11 and Table 7.8. 


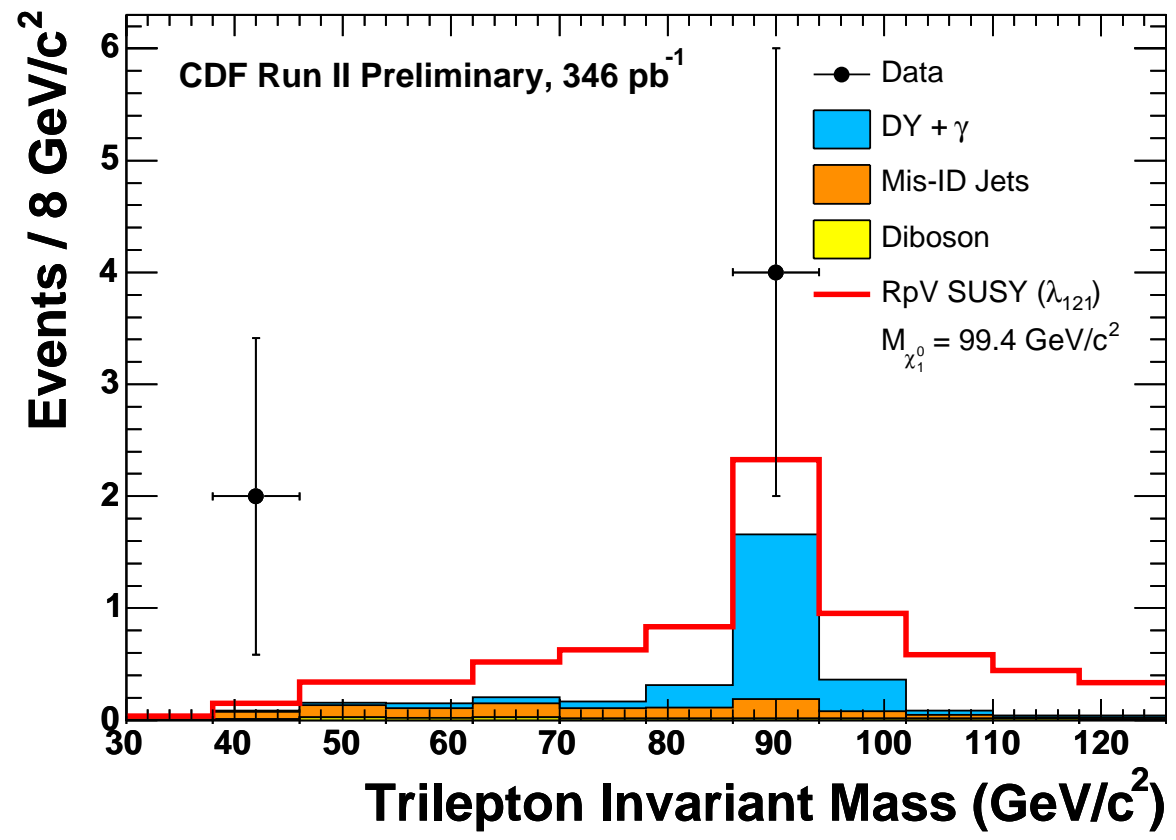

Figure 7.1: Combined invariant mass of the three identified leptons for the events within the trilepton signal samples. The $\not R_{p}$ SUSY signal $\left(M_{0}=250, M_{1 / 2}=260\right.$, $\tan \beta=5, A_{0}=0$, and $\mu>0$ ) is stacked on top of the backgrounds. 


\begin{tabular}{lcc}
\hline \multicolumn{3}{c}{ Trilepton Signal Samples $\left(\mathbf{3 4 6} \mathbf{~ p b}^{-1}\right)$} \\
\hline MC Sample & $\lambda_{121}(\mathbf{n o} \mu \mu l)$ & $\lambda_{122}($ no eel $)$ \\
\hline$Z / \gamma^{*}+\gamma$ & $2.1 \pm 0.7$ & $1.2 \pm 1.0$ \\
$W Z / \gamma^{*}$ & $0.17 \pm 0.01$ & $0.14 \pm 0.01$ \\
$Z / \gamma^{*} Z / \gamma^{*}$ & $0.066 \pm 0.008$ & $0.063 \pm 0.008$ \\
Jets misidentified as TCEs and LCEs & $0.34 \pm 0.17$ & $0.28 \pm 0.14$ \\
Jets misidentified as plug electrons & $0.16 \pm 0.08$ & $0.13 \pm 0.07$ \\
Jets misidentified as CMU muons & $0.02 \pm 0.01$ & $0.004 \pm 0.002$ \\
Jets misidentified as CMX muons & $0.02 \pm 0.01$ & $0.010 \pm 0.005$ \\
Jets misidentified as CMIO muons & $0.18 \pm 0.09$ & $0.07 \pm 0.04$ \\
Total mis-ID jets & $0.71 \pm 0.35$ & $0.49 \pm 0.25$ \\
\hline Total Background & $3.1 \pm 0.8$ & $1.9 \pm 1.0$ \\
\hline$\not R_{p}$ SUSY $\left(\lambda_{121}\right)$ & $4.3 \pm 0.4$ & - \\
$\not R_{p}$ SUSY $\left(\lambda_{122}\right)$ & -5 & $3.8 \pm 0.4$ \\
\hline Observed & 5 & 1 \\
\hline
\end{tabular}

Table 7.1: Comparison of expected numbers of background events and $\not R_{p}$ SUSY prediction within the trilepton signal sample. The $\not R_{p}$ SUSY samples are generated with the following parameters: $M_{0}=250, M_{1 / 2}=260, \tan \beta=5, A_{0}=0$, and $\mu>0$. 


\begin{tabular}{lc}
\hline \multicolumn{2}{c}{ 4+ Lepton Signal Sample $\left(\mathbf{3 4 6} \mathbf{p b}^{-1}\right)$} \\
\hline $\mathrm{Z} / \gamma^{*}+2 \gamma$ & $0.0001 \pm 0.0001$ \\
$\mathrm{Z} / \gamma^{*}+\mathrm{Z} / \gamma^{*}$ & $0.004 \pm 0.002$ \\
Total mis-ID jets & $0.004 \pm 0.003$ \\
\hline Total Background & $0.008 \pm 0.004$ \\
\hline$\not R_{p}$ SUSY $\left(\lambda_{121}\right)$ & $1.3 \pm 0.2$ \\
$\not R_{p}$ SUSY $\left(\lambda_{122}\right)$ & $1.3 \pm 0.2$ \\
\hline Observed & 0 \\
\hline
\end{tabular}

Table 7.2: Comparison of expected backgrounds and $\not R_{p}$ SUSY prediction within the four or more lepton signal sample. The $\not R_{p}$ SUSY samples are generated with the following parameters: $M_{0}=250, M_{1 / 2}=260, \tan \beta=5, A_{0}=0$, and $\mu>0$. 


\begin{tabular}{|l|l|l|l|}
\hline Date & $8 / 13 / 02$ & $12 / 12 / 02$ & $5 / 16 / 03$ \\
Run & 150820 & 155301 & 163009 \\
Event & 3985548 & 388905 & 110391 \\
Lepton 1 & TCE & TCE & TCE \\
Lepton 2 & TCE & TCE & LCE \\
Lepton 3 & CMIO* & TCE & TCE \\
Lepton $\mathrm{E}_{T} 1(\mathrm{GeV})$ & 21.2 & 40.3 & 29.8 \\
Lepton $\mathrm{E}_{T} 2(\mathrm{GeV})$ & 15.7 & 29.7 & 49.1 \\
Lepton $\mathrm{E}_{T} 3(\mathrm{GeV})$ & 10.1 & 20.0 & 13.2 \\
Lepton $\eta 1$ & -0.98 & 0.73 & -0.97 \\
Lepton $\eta 2$ & 0.34 & 0.29 & -0.78 \\
Lepton $\eta 3$ & 0.02 & 0.82 & -0.48 \\
Lepton $\phi 1(\mathrm{rad})$ & 4.83 & 1.40 & 4.62 \\
Lepton $\phi 2(\mathrm{rad})$ & 3.20 & 5.06 & 0.70 \\
Lepton $\phi 3(\mathrm{rad})$ & 3.49 & 3.64 & 3.60 \\
Lepton $\mathrm{Charge} 1$ & - & + & + \\
Lepton $\mathrm{Charge} 2$ & + & - & - \\
Lepton $\mathrm{Charge} 3$ & - & + & - \\
$\mathrm{M}_{\ell \ell} 1,2\left(\mathrm{GeV} / c^{2}\right)$ & 37.0 & 74.8 & 70.0 \\
$\mathrm{M}_{\ell \ell} 1,3\left(\mathrm{GeV} / c^{2}\right)$ & 23.5 & 51.5 & 21.0 \\
$\mathrm{M}_{\ell \ell} 2,3\left(\mathrm{GeV} / c^{2}\right)$ & 5.6 & 25.9 & 50.3 \\
$\mathrm{M}_{\ell \ell \ell}\left(\mathrm{GeV} / c^{2}\right)$ & 44.1 & 92.3 & 88.8 \\
E $_{T}(\mathrm{GeV})$ & 5.9 & 5.2 & 5.5 \\
\hline
\end{tabular}

Table 7.3: Kinematic properties of the first three events in the trilepton signal samples. (*) The CMIO does not pass through the calorimeter and may be a misidentified electron or jet. 


\begin{tabular}{|l|l|l|l|}
\hline Date & $6 / 26 / 03$ & $7 / 15 / 04$ & $7 / 24 / 04$ \\
Run & 165297 & 185201 & 185518 \\
Event & 5124167 & 5718803 & 23876 \\
Lepton 1 & CMX & TCE & TCE \\
Lepton 2 & CMX & TCE & TCE \\
Lepton 3 & Plug & Plug & LCE \\
Lepton $\mathrm{E}_{T} 1(\mathrm{GeV})$ & 21.5 & 62.9 & 23.4 \\
Lepton $\mathrm{E}_{T} 2(\mathrm{GeV})$ & 24.7 & 38.5 & 8.9 \\
Lepton $\mathrm{E}_{T} 3(\mathrm{GeV})$ & 12.5 & 13.3 & 6.5 \\
Lepton $\eta 1$ & -0.78 & 0.56 & 0.41 \\
Lepton $\eta 2$ & 0.85 & 0.79 & -0.35 \\
Lepton $\eta 3$ & -1.30 & -1.25 & -0.88 \\
Lepton $\phi 1(\mathrm{rad})$ & 2.81 & 1.75 & 2.03 \\
Lepton $\phi 2(\mathrm{rad})$ & 0.45 & 2.31 & 6.22 \\
Lepton $\phi 3(\mathrm{rad})$ & 4.58 & 2.29 & 4.60 \\
Lepton Charge 1 & + & + & - \\
Lepton Charge 2 & - & - & + \\
Lepton Charge 3 & + & - & + \\
$\mathrm{M}_{\ell \ell} 1,2\left(\mathrm{GeV} / c^{2}\right)$ & 59.7 & 29.7 & 28.3 \\
$\mathrm{M}_{\ell \ell} 1,3\left(\mathrm{GeV} / c^{2}\right)$ & 27.2 & 62.9 & 29.0 \\
$\mathrm{M}_{\ell \ell} 2,3\left(\mathrm{GeV} / c^{2}\right)$ & 58.0 & 55.3 & 11.9 \\
$\mathrm{M}_{\ell \ell \ell}\left(\mathrm{GeV} / c^{2}\right)$ & 87.6 & 88.8 & 42.2 \\
E $_{T}(\mathrm{GeV})$ & 23.66 & 10.1 & 12.3 \\
\hline
\end{tabular}

Table 7.4: Kinematic properties of the last three events in the trilepton signal samples. 

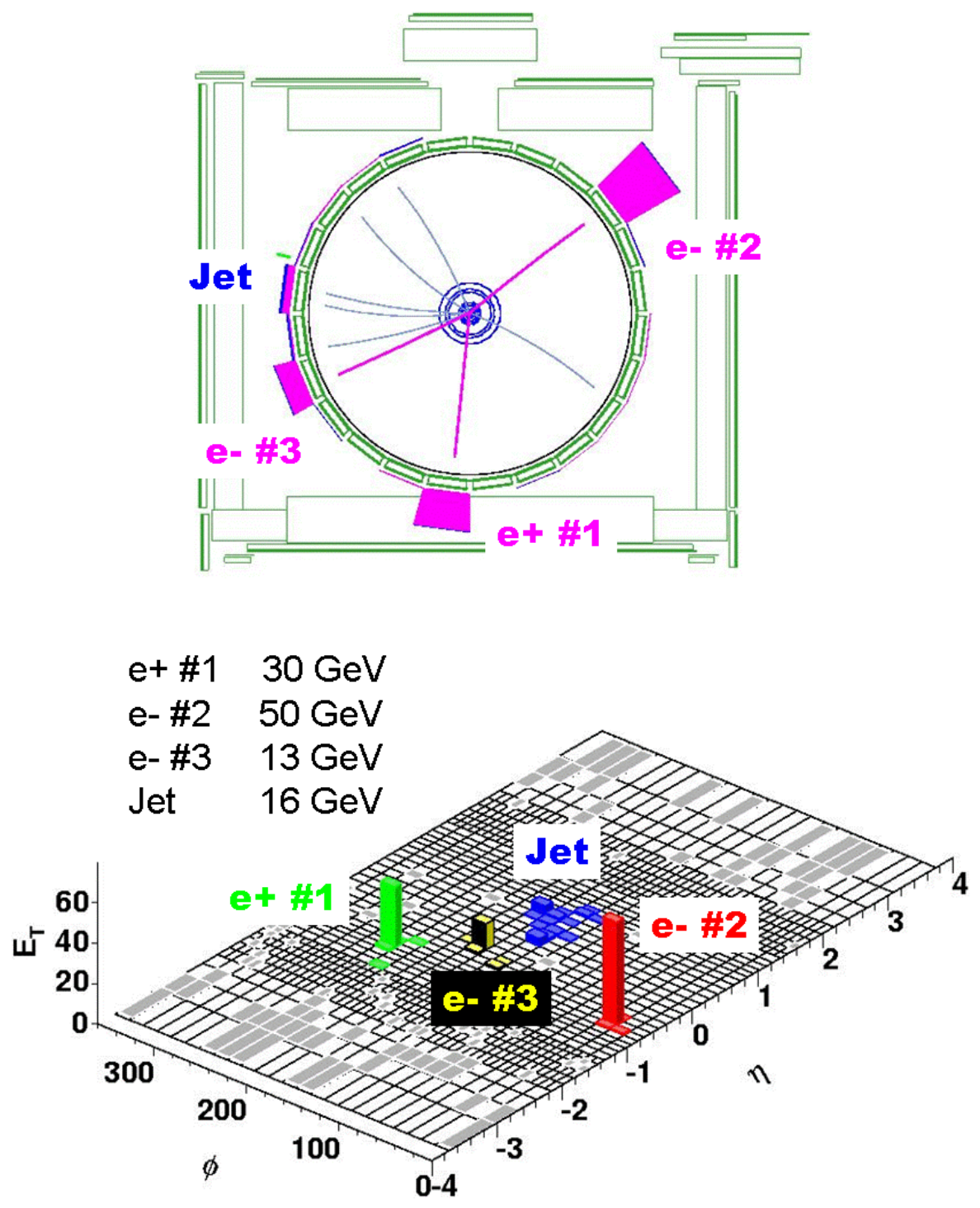

Figure 7.2: Event displays for trilepton signal event Run=163009, Event=110391. The top plot shows a cross section of the CDF detector including all tracks above $0.5 \mathrm{GeV} / c$ and calorimeter energy within the EM (purple) and the hadronic (blue) sections. The bottom plot displays the energy within each calorimeter cluster in the $\eta-\phi$ plane. The grid represents the calorimeter towers. 


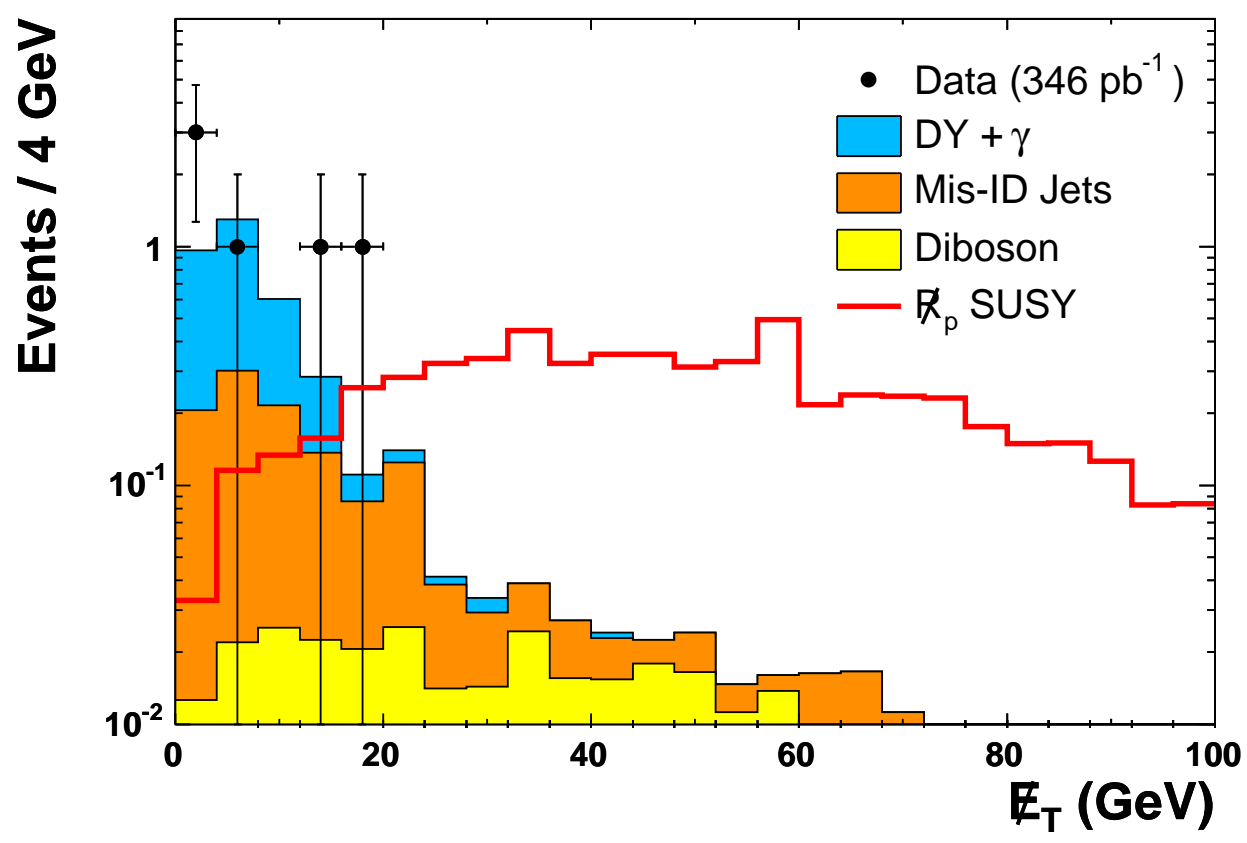

Figure 7.3: Missing transverse energy distribution of the events within the trilepton signal samples. The $R_{p}$ SUSY signal $\left(M_{0}=250, M_{1 / 2}=260, \tan \beta=5\right.$, $A_{0}=0$, and $\left.\mu>0\right)$ is plotted independently of the backgrounds. 

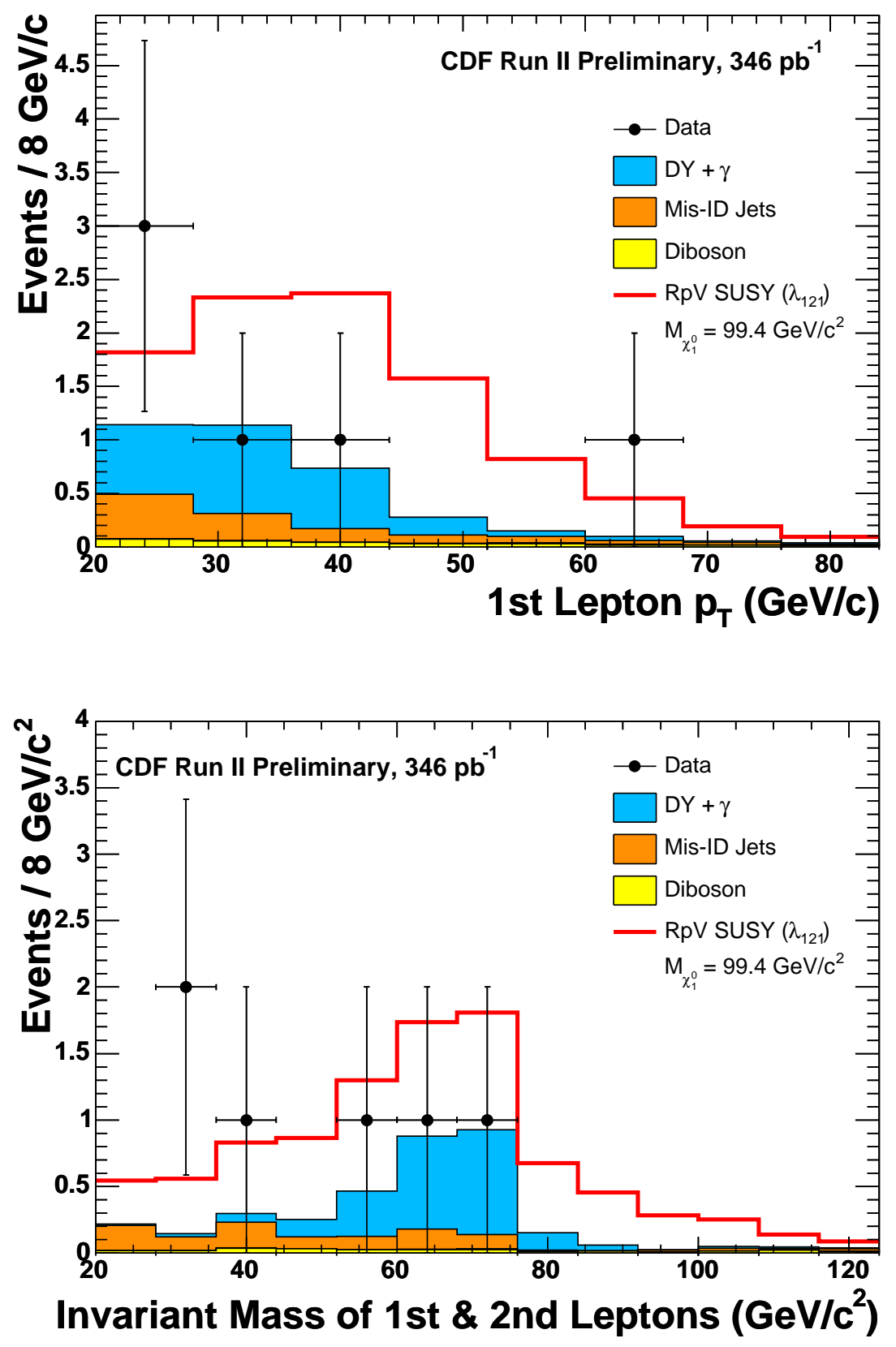

Figure 7.4: Invariant mass of the first two leptons (top) and 1st lepton $p_{T}$ (bottom) distributions for the events within the trilepton signal samples. The $R_{p}$ SUSY signal $\left(M_{0}=250, M_{1 / 2}=260, \tan \beta=5, A_{0}=0\right.$, and $\left.\mu>0\right)$ is stacked on top of the backgrounds. 

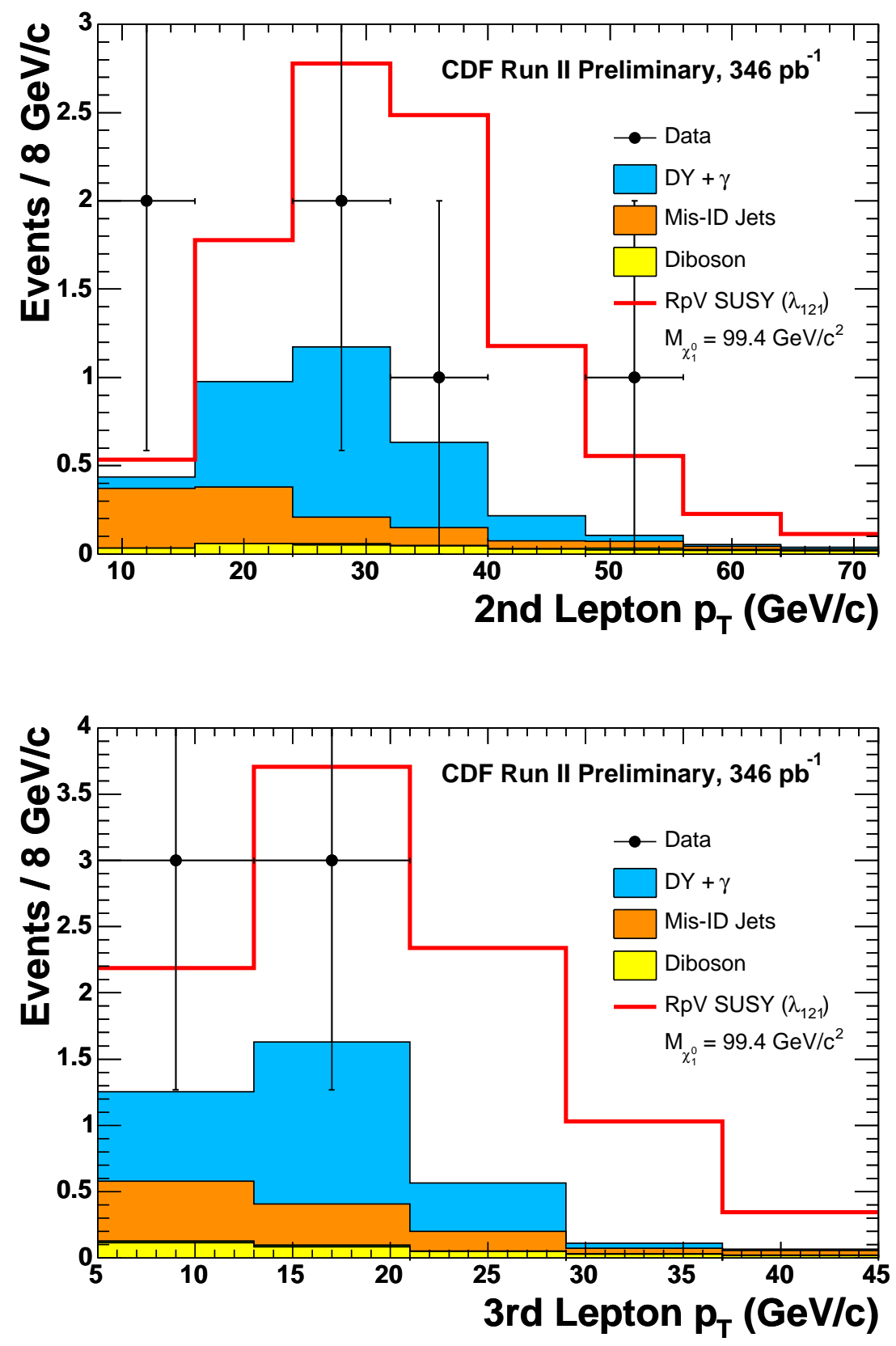

Figure 7.5: 2nd lepton $\mathrm{p}_{T}$ (top) and 3rd lepton $\mathrm{p}_{T}$ (bottom) distributions for the events within the trilepton signal samples. The $\not R_{p}$ SUSY signal $\left(M_{0}=250\right.$, $M_{1 / 2}=260, \tan \beta=5, A_{0}=0$, and $\left.\mu>0\right)$ is stacked on top of the backgrounds. 

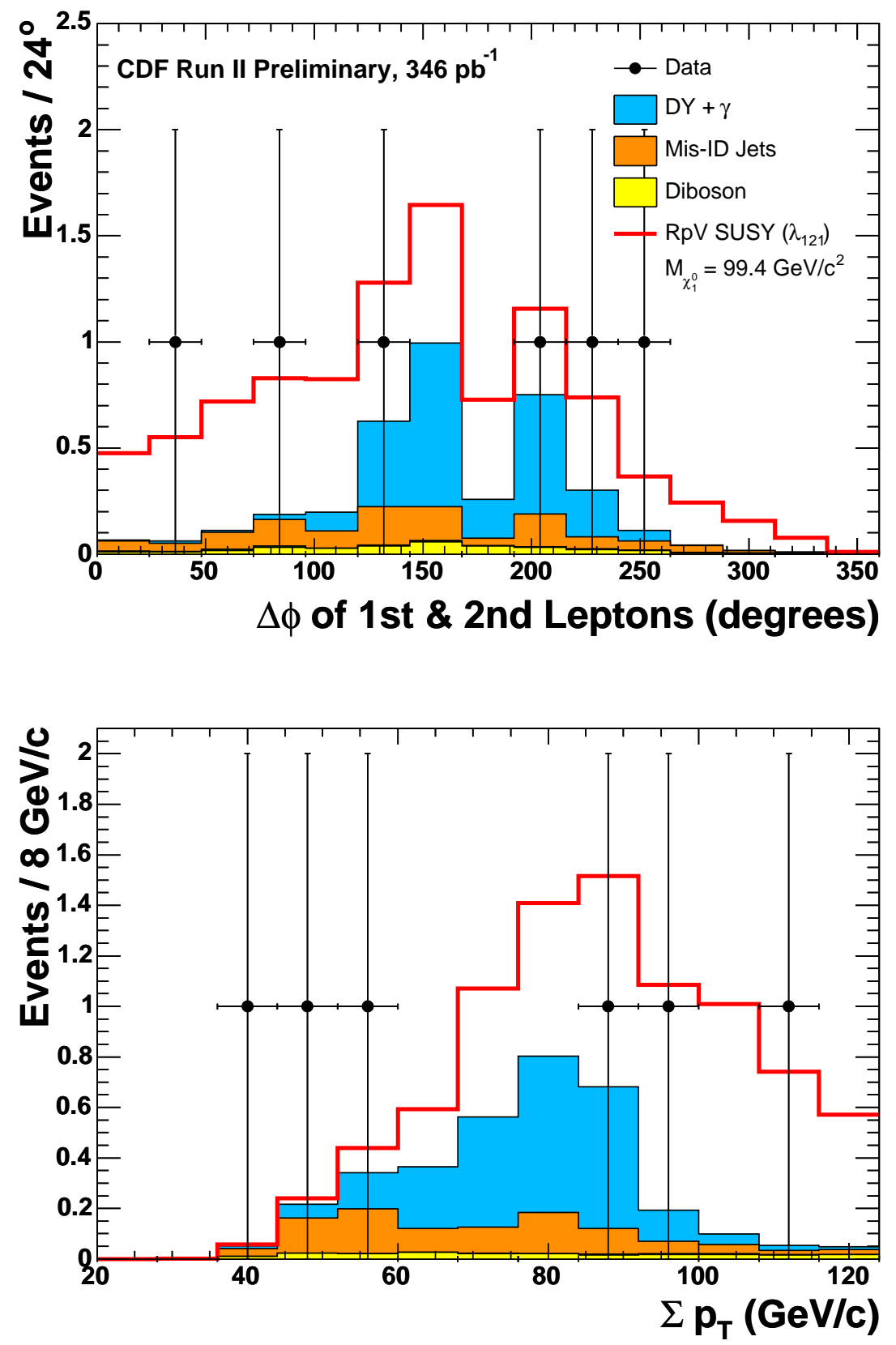

Figure 7.6: $\Delta \phi$ of the first two leptons (top) and sum of the lepton $\mathrm{p}_{T}$ (bottom) distributions for the events within the trilepton signal samples. The $\not k_{p}$ SUSY signal $\left(M_{0}=250, M_{1 / 2}=260, \tan \beta=5, A_{0}=0\right.$, and $\left.\mu>0\right)$ is stacked on top of the backgrounds. 


\begin{tabular}{|c|c|c|c|c|c|}
\hline \multicolumn{3}{|c|}{ Inputs } & \multicolumn{3}{|c|}{ Outputs } \\
\hline$\lambda$ & $\mu$ & $\begin{array}{c}M_{1 / 2} \\
\left(\mathrm{GeV} / c^{2}\right)\end{array}$ & $\begin{array}{l}\text { Expected Limit } \\
\qquad(\mathrm{pb})\end{array}$ & $\begin{array}{l}\text { Observed Limit } \\
\qquad(\mathrm{pb})\end{array}$ & $\begin{array}{c}\sigma \mathrm{NLO} \\
(\mathrm{pb})\end{array}$ \\
\hline 121 & $>0$ & 130 & 0.375 & 0.452 & 7.29 \\
\hline 121 & $>0$ & 230 & 0.091 & 0.111 & 0.269 \\
\hline 121 & $>0$ & 240 & 0.088 & 0.108 & 0.213 \\
\hline 121 & $>0$ & 250 & 0.089 & 0.107 & 0.164 \\
\hline 121 & $>0$ & 260 & 0.091 & 0.110 & 0.128 \\
\hline 121 & $>0$ & 270 & 0.095 & 0.116 & 0.101 \\
\hline 121 & $>0$ & 280 & 0.092 & 0.111 & 0.082 \\
\hline 121 & $>0$ & 290 & 0.092 & 0.112 & 0.063 \\
\hline 121 & $<0$ & 110 & 0.708 & 0.866 & 4.99 \\
\hline 121 & $<0$ & 200 & 0.123 & 0.150 & 0.322 \\
\hline 121 & $<0$ & 210 & 0.116 & 0.141 & 0.251 \\
\hline 121 & $<0$ & 220 & 0.103 & 0.125 & 0.197 \\
\hline 121 & $<0$ & 230 & 0.095 & 0.115 & 0.154 \\
\hline 121 & $<0$ & 240 & 0.093 & 0.112 & 0.124 \\
\hline 121 & $<0$ & 250 & 0.092 & 0.112 & 0.098 \\
\hline 121 & $<0$ & 260 & 0.093 & 0.113 & 0.077 \\
\hline 121 & $<0$ & 270 & 0.092 & 0.112 & 0.064 \\
\hline
\end{tabular}

Table 7.5: Expected and observed cross section upper limits determined at the $95 \%$ confidence level combining the 3 lepton and $\geq 4$ lepton signal samples for $\lambda_{121}$. 


\begin{tabular}{ccc|ccc}
\hline \multicolumn{2}{c|}{ Inputs } & \multicolumn{3}{c}{ Outputs } \\
\hline$\lambda$ & $\mu$ & $M_{1 / 2}$ & Expected Limit & Observed Limit & $\sigma$ NLO \\
& & $\left(\mathrm{GeV} / c^{2}\right)$ & $(\mathrm{pb})$ & $(\mathrm{pb})$ & $(\mathrm{pb})$ \\
\hline 122 & $>0$ & 130 & 0.340 & 0.285 & 7.29 \\
122 & $>0$ & 240 & 0.084 & 0.071 & 0.213 \\
122 & $>0$ & 250 & 0.085 & 0.072 & 0.164 \\
122 & $>0$ & 260 & 0.085 & 0.072 & 0.128 \\
122 & $>0$ & 270 & 0.086 & 0.073 & 0.101 \\
122 & $>0$ & 280 & 0.082 & 0.069 & 0.082 \\
122 & $>0$ & 290 & 0.083 & 0.070 & 0.063 \\
122 & $>0$ & 300 & 0.080 & 0.068 & 0.050 \\
\hline 122 & $<0$ & 110 & 0.532 & 0.499 & 4.49 \\
122 & $<0$ & 230 & 0.087 & 0.074 & 0.154 \\
122 & $<0$ & 240 & 0.084 & 0.071 & 0.124 \\
122 & $<0$ & 250 & 0.083 & 0.070 & 0.098 \\
122 & $<0$ & 260 & 0.084 & 0.071 & 0.077 \\
122 & $<0$ & 270 & 0.082 & 0.070 & 0.064 \\
122 & $<0$ & 280 & 0.082 & 0.070 & 0.050 \\
\hline & & & & & \\
\hline
\end{tabular}

Table 7.6: Expected and observed cross section upper limits determined at the $95 \%$ confidence level combining the 3 lepton and $\geq 4$ lepton signal samples for $\lambda_{122}$. 


\begin{tabular}{ccccc}
\hline \# Signal Samples & \# Leptons & $\lambda$ & $\begin{array}{c}\text { Expected Limit } \\
(\mathrm{pb})\end{array}$ & $\begin{array}{c}\text { Observed Limit } \\
(\mathrm{pb})\end{array}$ \\
\hline 1 & 3 & 121 & 0.131 & 0.165 \\
1 & $\geq 4$ & 121 & 0.250 & 0.249 \\
1 & $\geq 3$ & 121 & 0.099 & 0.125 \\
2 & $3, \geq 4$ & 121 & 0.091 & 0.110 \\
\hline 1 & 3 & 122 & 0.123 & 0.101 \\
1 & $\geq 4$ & 122 & 0.232 & 0.231 \\
2 & $\geq 3$ & 122 & 0.091 & 0.075 \\
\hline
\end{tabular}

Table 7.7: Benefits of splitting the signal sample into two, one with exactly three leptons and one with four or more leptons. Expected and observed cross section limits are determined at $95 \% \mathrm{CL}$ for $M_{0}=250 \mathrm{GeV} / c^{2}, M_{1 / 2}=260 \mathrm{GeV} / c^{2}$, $\mu>0$, and $A_{0}=0$. 

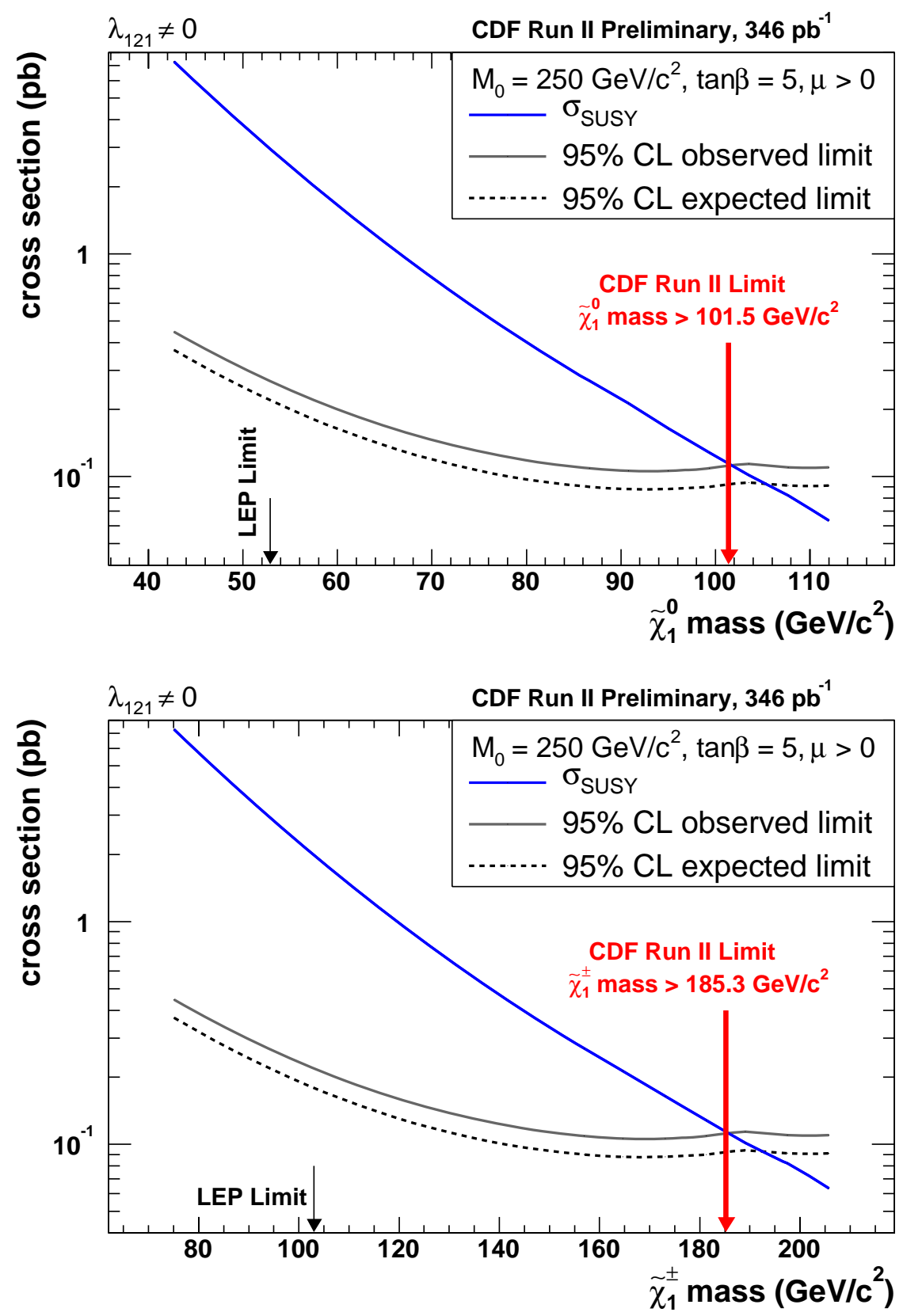

Figure 7.7: $\not \ell_{p}$ SUSY mass lower limits for $\lambda_{121}$ and $\mu>0$. Expected limits: $\operatorname{M}\left(\tilde{\chi}_{1}^{0}\right)$ $>99.8 \mathrm{GeV} / c^{2}, \mathrm{M}\left(\tilde{\chi}_{1}^{ \pm}\right)>182.1 \mathrm{GeV} / c^{2}$. Observed limits: $\mathrm{M}\left(\tilde{\chi}_{1}^{0}\right)>94.8 \mathrm{GeV} / c^{2}$, $\mathrm{M}\left(\tilde{\chi}_{1}^{ \pm}\right)>172.6 \mathrm{GeV} / c^{2}$. 

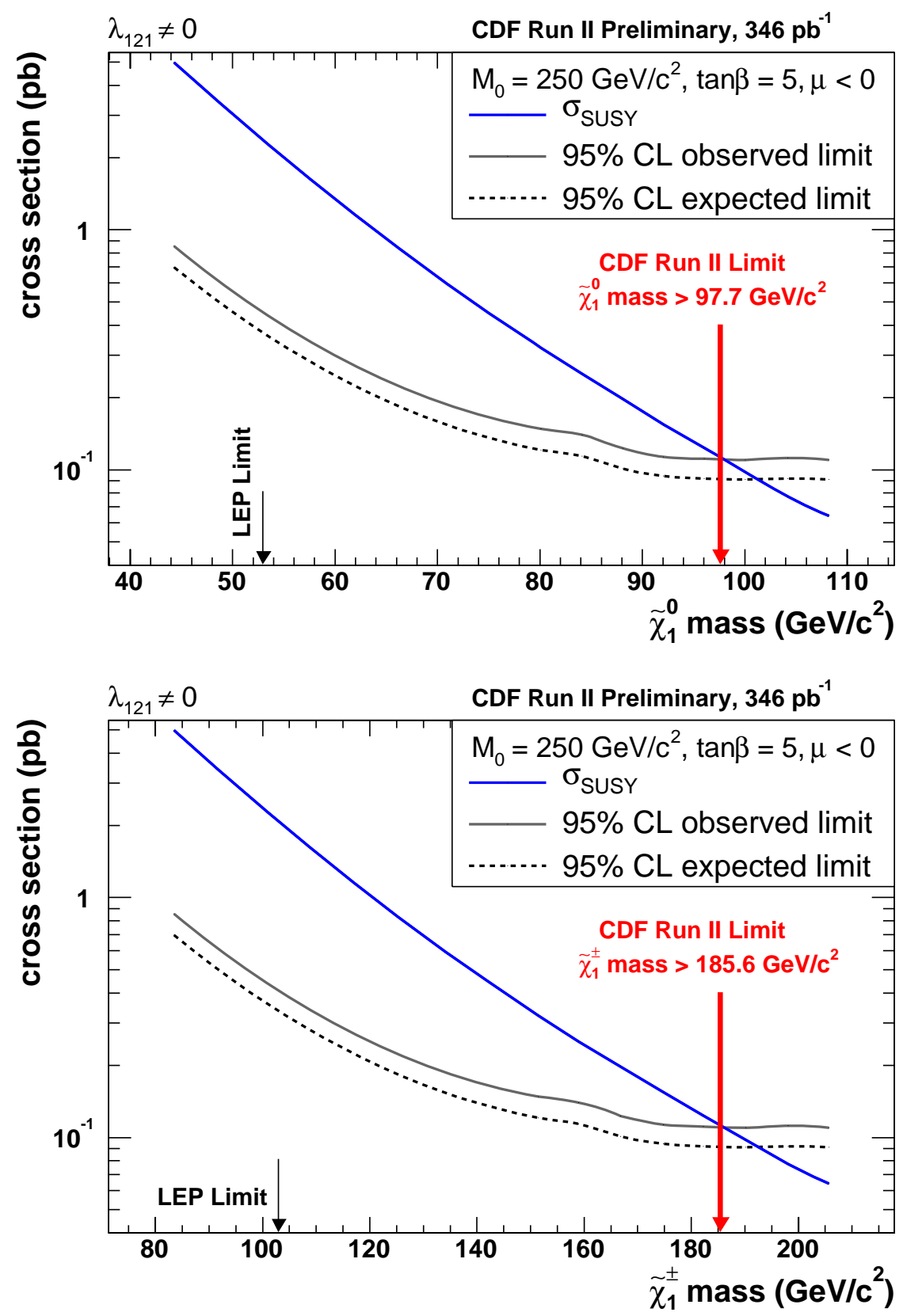

Figure 7.8: $\not \ell_{p}$ SUSY mass lower limits for $\lambda_{121}$ and $\mu<0$. Expected limits: $\operatorname{M}\left(\tilde{\chi}_{1}^{0}\right)$ $>95.5 \mathrm{GeV} / c^{2}, \mathrm{M}\left(\tilde{\chi}_{1}^{ \pm}\right)>181.3 \mathrm{GeV} / c^{2}$. Observed limits: $\mathrm{M}\left(\tilde{\chi}_{1}^{0}\right)>88.4 \mathrm{GeV} / c^{2}$, $\mathrm{M}\left(\tilde{\chi}_{1}^{ \pm}\right)>167.4 \mathrm{GeV} / c^{2}$. 

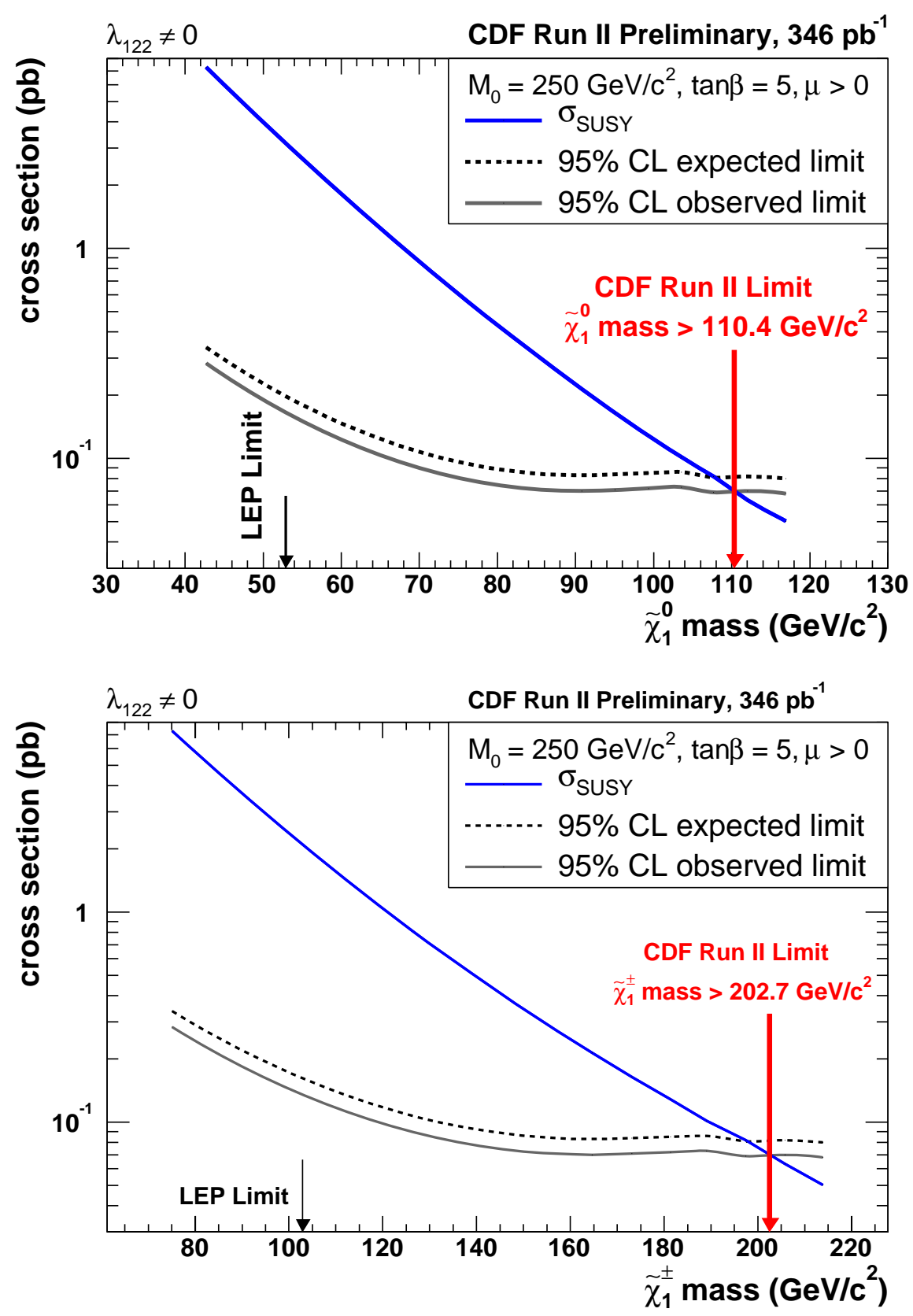

Figure 7.9: $R_{p}$ SUSY mass lower limits for $\lambda_{122}$ and $\mu>0$. Expected limits: $\mathrm{M}\left(\tilde{\chi}_{1}^{0}\right)>103.6 \mathrm{GeV} / c^{2}, \mathrm{M}\left(\tilde{\chi}_{1}^{ \pm}\right)>189.2 \mathrm{GeV} / c^{2}$. Observed limits: $\mathrm{M}\left(\tilde{\chi}_{1}^{0}\right)>$ $108.4 \mathrm{GeV} / c^{2}, \mathrm{M}\left(\tilde{\chi}_{1}^{ \pm}\right)>198.7 \mathrm{GeV} / c^{2}$. 

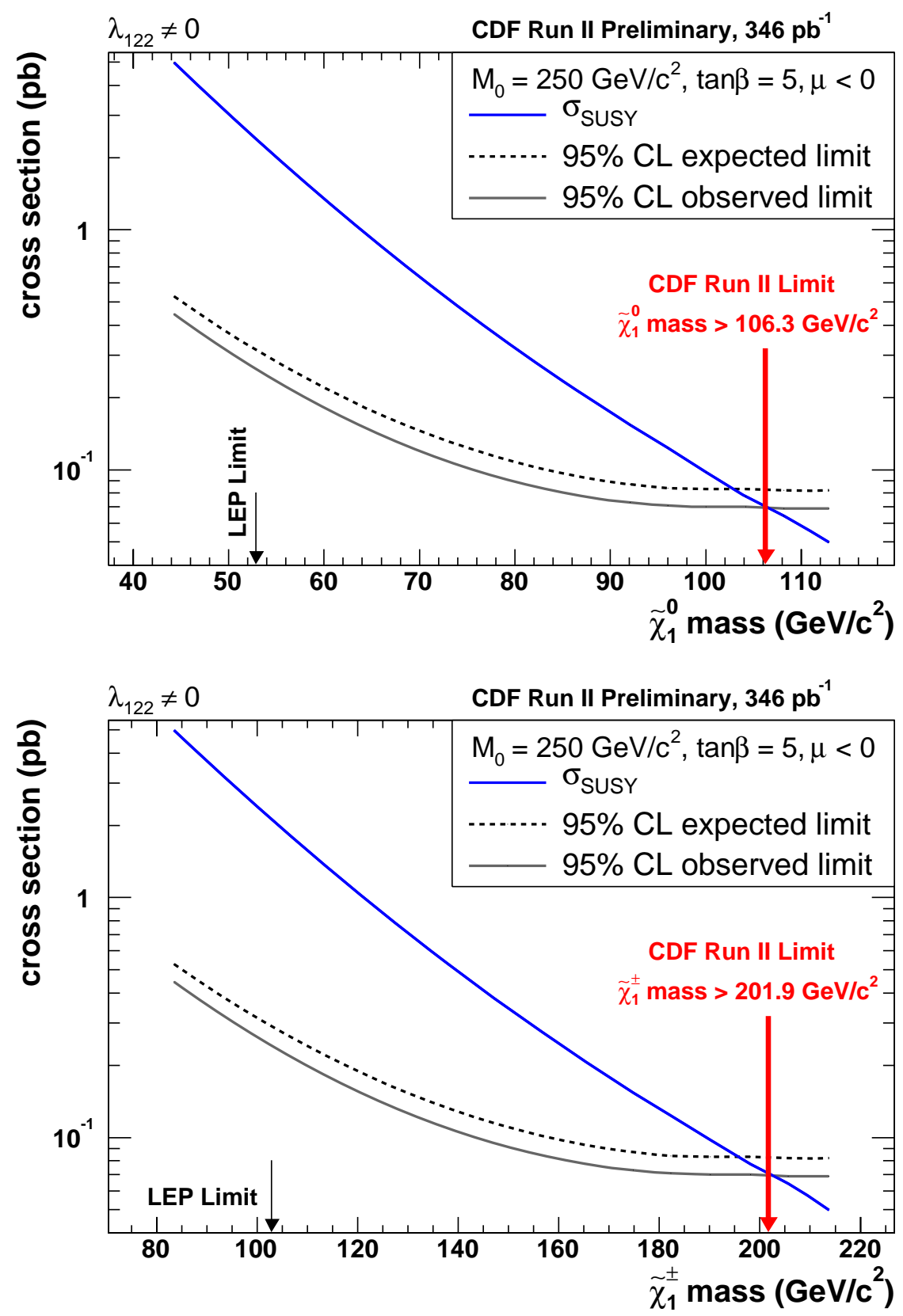

Figure 7.10: $\mathbb{R}_{p}$ SUSY mass lower limits for $\lambda_{122}$ and $\mu<0$. Expected limits: $\mathrm{M}\left(\tilde{\chi}_{1}^{0}\right)>100.2 \mathrm{GeV} / c^{2}, \mathrm{M}\left(\tilde{\chi}_{1}^{ \pm}\right)>190.4 \mathrm{GeV} / c^{2}$. Observed limits: $\mathrm{M}\left(\tilde{\chi}_{1}^{0}\right)>$ $103.3 \mathrm{GeV} / c^{2}, \mathrm{M}\left(\tilde{\chi}_{1}^{ \pm}\right)>196.5 \mathrm{GeV} / c^{2}$. 


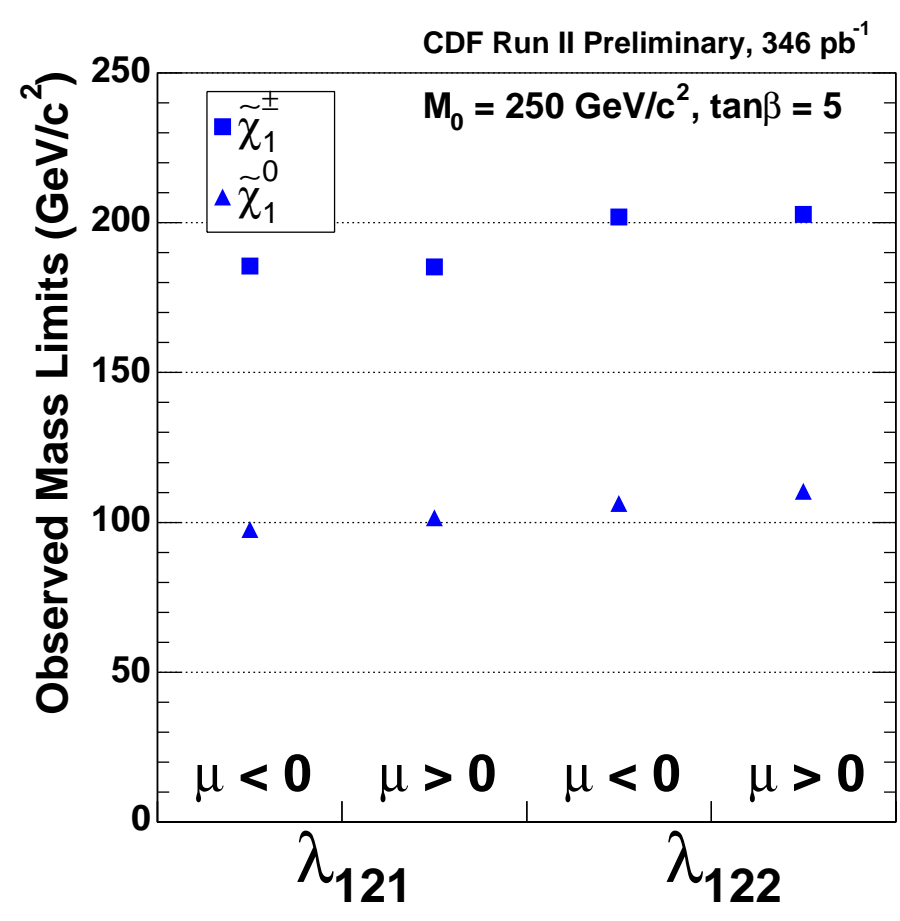

Figure 7.11: Observed 95\% CL limits on the $\tilde{\chi}_{1}^{0}$ and $\tilde{\chi}_{1}^{ \pm}$masses for $M_{0}=250 \mathrm{GeV} / c^{2}, \tan \beta=5, A_{0}=0$.

\begin{tabular}{ccccc}
\hline & \multicolumn{2}{c}{$\mathrm{M}\left(\tilde{\chi}_{1}^{0}\right)$} & {$\left[\mathrm{GeV} / c^{2}\right]$} & \multicolumn{2}{c}{$\mathrm{M}\left(\tilde{\chi}_{1}^{ \pm}\right)$} & {$\left[\mathrm{GeV} / c^{2}\right]$} \\
& exp. & obs. & exp. & obs. \\
\hline$\lambda_{121}, \mu>0$ & 105.0 & 101.5 & 191.9 & 185.3 \\
$\lambda_{121}, \mu<0$ & 101.1 & 97.7 & 192.2 & 185.6 \\
$\lambda_{122}, \mu>0$ & 107.7 & 110.4 & 197.5 & 202.7 \\
$\lambda_{122}, \mu<0$ & 102.7 & 106.3 & 195.3 & 201.9 \\
\hline
\end{tabular}

Table 7.8: Expected and observed 95\% CL limits on the $\tilde{\chi}_{1}^{0}$ and $\tilde{\chi}_{1}^{ \pm}$masses for $M_{0}=250 \mathrm{GeV} / c^{2}, \tan \beta=5, A_{0}=0$. 


\section{CHAPTER 8}

\section{Conclusions}

This thesis describes a search for physics beyond the standard model (SM) by looking for an excess of events with three or more charged leptons at CDF using an integrated luminosity of $346 \mathrm{pb}^{-1}$. The search is based on an $k_{p}$ minimal supergravity model, but is sensitive to other new physics models since there are no event selection requirements based on missing energy or jets. The analysis procedures are studied using data and simulated events and validated through control samples. In the end, no significant evidence for new physics is detected. In

order for $\not_{p}$ SUSY to be consistent with these findings, the superpartner masses must be larger than certain values. Lower bounds on the $\tilde{\chi}_{1}^{0}$ mass are placed between 97.7 and $106.3 \mathrm{GeV} / c^{2}$ and on the $\tilde{\chi}_{1}^{ \pm}$mass between 185.3 and 202.7 $\mathrm{GeV} / c^{2}$ valid for $3 \times 10^{-3} \lesssim \lambda_{12 k} \lesssim 0.14$. These limits are significantly better than those placed by the LEP experiment of $\sim 53 \mathrm{GeV} / c^{2}$ on the $\tilde{\chi}_{1}^{0}$ mass and $103 \mathrm{GeV} / c^{2}$ on the $\tilde{\chi}_{1}^{ \pm}$mass [70]. The results are similar but not as restrictive as the DØ limits using $360 \mathrm{pb}^{-1}$ of $115-119 \mathrm{GeV} / \mathrm{c}^{2}$ and $229-234 \mathrm{GeV} / \mathrm{c}^{2}$ for the $\tilde{\chi}_{1}^{0}$ and $\tilde{\chi}_{1}^{ \pm}$masses, respectively [28]. 


\section{APPENDIX A}

\section{Almost Discovering Supersymmetry}

In this thesis, I include the essential ingredients that are necessary to understand why the analysis is important, how it is carried out, and what the results are. But this is not the whole story. Another aspect of the analysis, which is not included in the main text is all the pitfalls and wrong turns along the way. This is left out intentionally, not to make it seem like I am perfect and make no mistakes, but because if it was included the thesis could easily be five times as

long. In some sense, it is a shame that the mistakes are not presented, because that knowledge could help other people avoid repeating the same undesired consequences. Therefore, in this section I describe my largest mistake: how I almost "discovered" supersymmetry, how the mistake was found, and how it was dealt with.

\section{A.1 Introduction}

To properly explain what happened, I need to first go over a few of the analysis techniques that were previously described within this thesis, in particular the event selection and validation. The event selection defines what events are included within the signal samples. As described in Sec. 6.1, choosing the event selection criteria is a process where I attempt to maximize the $k_{p}$ SUSY signal expectation compared to the background prediction. In order to ensure the ac- 
curacy of the SM background prediction and the $\not_{p}$ SUSY signal expectation in the signal samples, a method of validation is required. Control samples work well for this purpose since they involve the same procedure that is used to find the final result: comparing a background prediction to the data. The most effective control samples have the same dominant backgrounds as those within the signal sample and contain enough events so that statistical uncertainty does not become a large factor. Most of the control samples used to validate the analysis contain only two leptons and therefore the background prediction are not dominated by the same processes as in the signal samples. Control samples with three leptons are the most effective, yet have very little statistics, which makes it hard to judge whether there is adequate agreement between data and the background prediction. The problem that I am about to discuss, manifested itself due to an inaccuracy in the background prediction which was not uncovered by any of the control samples used at the time.

The process of validation started with comparing the background expectation to data in the dilepton control samples only, where the largest background is due to DY events. Once this was completed, I moved on to the trilepton control sample, consisting of events that have two opposite sign charged leptons within the $Z$ boson mass range, $76<\mathrm{M}_{\ell^{+} \ell^{-}}<106 \mathrm{GeV} / c^{2}$ ( $Z$ veto cut). At this point, the $160^{\circ}<\Delta \phi<200^{\circ}$ requirement on opposite sign leptons was not included in the event selection. It was only added later since a preliminary plot of $\Delta \phi$ in the trilepton control sample (Fig. A.1) showed a large difference between the distributions of the $\not_{p}$ SUSY and SM background. With the event selection finalized, I reexamined the trilepton control sample and saw good agreement, as shown in Table A.1. In this control sample, roughly half of the background prediction is composed of events with jets misidentified as leptons while the other half is composed of $Z / \gamma^{*}+\gamma$ events. Since the variation in the control sample is 
reasonable given the small statistics, it was determined that I was ready to look in the signal samples.

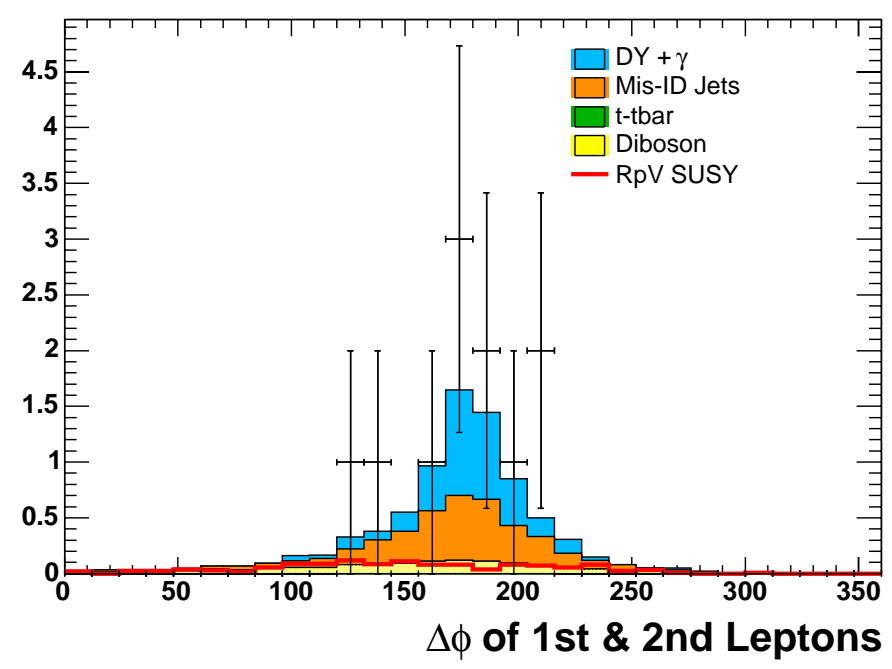

Figure A.1: Preliminary $\Delta \phi$ distribution including all trilepton events that fail the $Z$ veto cut.

\section{A.2 Discovery?}

Within the $\lambda_{121}$ trilepton signal sample, I expected $3.5 \pm 0.9$ events and observed 9! More information is shown in Table A.2. The probability of observing 9 or more events with 3.5 expected events of background is $\sim 1 \%$. Clearly, either I discovered physics beyond the SM, there was an improbable fluctuation of the background, or I made a mistake. Initial indications pointed to the latter conclusion. For instance, there were a couple of clues suggesting that the excess in data in the signal sample was due to $Z / \gamma^{*}+\gamma$ events, such as electrons barely passing the photon conversion requirements and missing silicon track hits, which could occur if a photon converts outside the silicon subdetector. Once it was realized that I 


\begin{tabular}{ccc}
\hline Control Sample & Background Prediction & Data \\
\hline$e e \ell$ & $8.1 \pm 2.4$ & 11 \\
$e \mu \ell$ & $0.8 \pm 0.2$ & 1 \\
$\mu \mu \ell$ & $4.3 \pm 1.4$ & 2 \\
$\ell \ell \ell$ & $13.2 \pm 4.2$ & 14 \\
\hline
\end{tabular}

Table A.1: Preliminary comparison between the background prediction and data for all trilepton events that fail the $Z$ veto cut. $\ell$ denotes electrons or muons.

did not discover supersymmetry, it was important to devise a plan to prevent my knowledge gained by looking at the data events from influencing the final result.

\section{A.3 The $\Delta \phi$ Control Sample}

Previously, the only trilepton control sample included events that failed the $Z$ veto cut. Now that the $\Delta \phi$ requirement had been introduced, another possible control sample existed: one that contains trilepton events failing the $\Delta \phi$ requirement, but passing the $Z$ veto cut. This sample was not previously studied purely due to an oversight, since this criteria was added late in the analysis. The data in this sample contains many more events than predicted from previous background estimates, as shown in Table A.3. In hindsight, it would have been nice to look at this control sample sooner, since the $Z / \gamma^{*}+\gamma$ background is at least twice as large as the misidentified jet background in the $\Delta \phi$ control sample, the same property seen in the trilepton signal samples, as shown in Table 7.1. On the bright side, the $\Delta \phi$ trilepton control sample does provide a method for deciding how to change the event selection while leaving the result unbiased. To prevent bias, all subsequent changes to the event selection must be based on improving 


\begin{tabular}{ccc}
\hline \multicolumn{3}{c}{ Signal Events } \\
\hline Sample & Trilepton $\left(\lambda_{121}\right)$ & Trilepton $\left(\lambda_{122}\right)$ \\
\hline$Z / \gamma^{*}+\gamma$ & $2.3 \pm 0.4$ & $1.2 \pm 0.2$ \\
Total mis-ID jets & $0.9 \pm 0.4$ & $0.4 \pm 0.2$ \\
$W Z / \gamma^{*}$ & $0.21 \pm 0.03$ & $0.13 \pm 0.02$ \\
$Z / \gamma^{*} Z / \gamma^{*}$ & $0.10 \pm 0.01$ & $0.070 \pm 0.007$ \\
\hline Total Background & $3.5 \pm 0.9$ & $1.8 \pm 0.5$ \\
\hline$\not k_{p}$ SUSY $\left(\lambda_{121}\right)$ & $5.1 \pm 0.6$ & - \\
$\not k_{p}$ SUSY $\left(\lambda_{122}\right)$ & - & $4.3 \pm 0.5$ \\
\hline Data & 9 & 2 \\
\hline
\end{tabular}

Table A.2: Preliminary comparison between the background prediction and data within the trilepton signal samples.

the agreement within the $\Delta \phi$ trilepton control sample without looking at how the changes affect the signal samples. Also I chose to not look at the data in the four or more lepton signal sample until resolving this issue in the trilepton signal sample.

\section{A.4 The Trilepton Invariant Mass Requirement}

One important question remained to be answered: How could I prove that the excess within the $\Delta \phi$ control sample is due to $Z / \gamma^{*}+\gamma$ events? $Z / \gamma^{*}+\gamma$ events may be produced in two ways at the Tevatron, either through initial state radiation (ISR) or final state radiation (FSR), shown in Fig. A.2. In the simulation, a majority of these events are due to FSR since the cross section is larger and more ISR events are rejected by the $Z$ veto cut. A signature of $Z / \gamma^{*}+\gamma$ events 


\begin{tabular}{|c|c|c|c|}
\hline Sample & eel & $\mu \mu \ell$ & $\ell \ell \ell$ \\
\hline$Z / \gamma^{*}+\gamma$ & $1.5 \pm 0.4$ & $0.5 \pm 0.2$ & $2.2 \pm 0.6$ \\
\hline Total mis-ID jets & $0.2 \pm 0.1$ & $0.2 \pm 0.1$ & $0.4 \pm 0.2$ \\
\hline$W Z / \gamma^{*}$ & $0.046 \pm 0.005$ & $0.017 \pm 0.004$ & $0.06 \pm 0.01$ \\
\hline$Z / \gamma^{*} Z / \gamma^{*}$ & $0.021 \pm 0.004$ & $0.015 \pm 0.003$ & $0.03 \pm 0.01$ \\
\hline Total Background & $1.8 \pm 0.8$ & $0.7 \pm 0.3$ & $2.7 \pm 0.9$ \\
\hline$\not R_{p} \operatorname{SUSY}\left(\lambda_{121}\right)$ & $0.7 \pm 0.1$ & $0.02 \pm 0.01$ & $0.8 \pm 0.2$ \\
\hline$\not R_{p} \operatorname{SUSY}\left(\lambda_{122}\right)$ & $0.17 \pm 0.04$ & $0.4 \pm 0.1$ & $0.6 \pm 0.2$ \\
\hline Data & 4 & 4 & 8 \\
\hline
\end{tabular}

Table A.3: Preliminary comparison between the background prediction and data for trilepton events which fail the $\Delta \phi$ requirement, yet pass the $Z$ veto cut.

produced through FSR is that the energy of the two charged leptons and the photon should add up to the energy of the $Z$. Therefore, the invariant mass of the three reconstructed leptons should be close to the $Z$ mass as well, as shown for the MC simulation in Fig. A.3. By rejecting events where there are two opposite sign lepton plus a third electron with $76<\mathrm{M}_{\ell^{+} \ell^{-} e}<106 \mathrm{GeV} / c^{2}$, all but one of the initial 8 events observed in the $\Delta \phi$ trilepton control sample are removed (see Table A.4), indicating that these are very probably $Z / \gamma^{*}+\gamma$ events.

\section{A.5 $Z / \gamma^{*}+\gamma$ Background Prediction}

The results of the previous section, leads to another important question: If the excess events are due to $Z / \gamma^{*}+\gamma$ production, then why were they not included in the background prediction? The only logical conclusion, was that my $Z / \gamma^{*}+\gamma$ prediction was underestimated. Since this prediction is calculated using purely 
a)

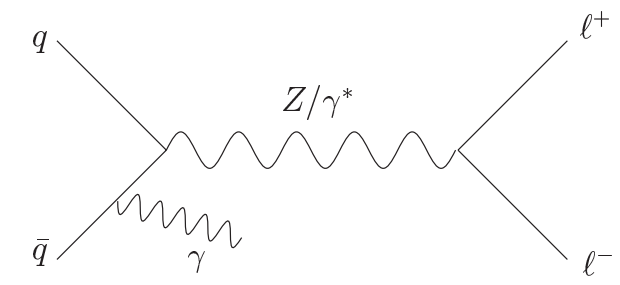

b)

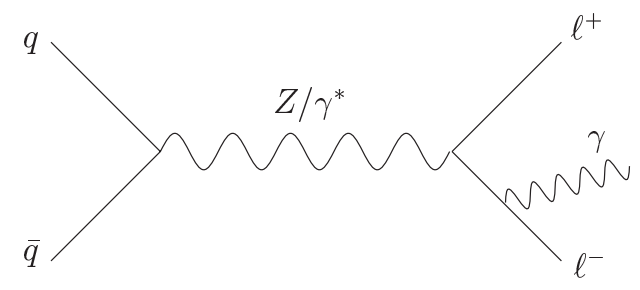

Figure A.2: $Z / \gamma^{*}+\gamma \rightarrow \ell^{+} \ell^{-}+\gamma$ production at the Tevatron. The $\gamma$ can be produced through a) initial state radiation or b) final state radiation.

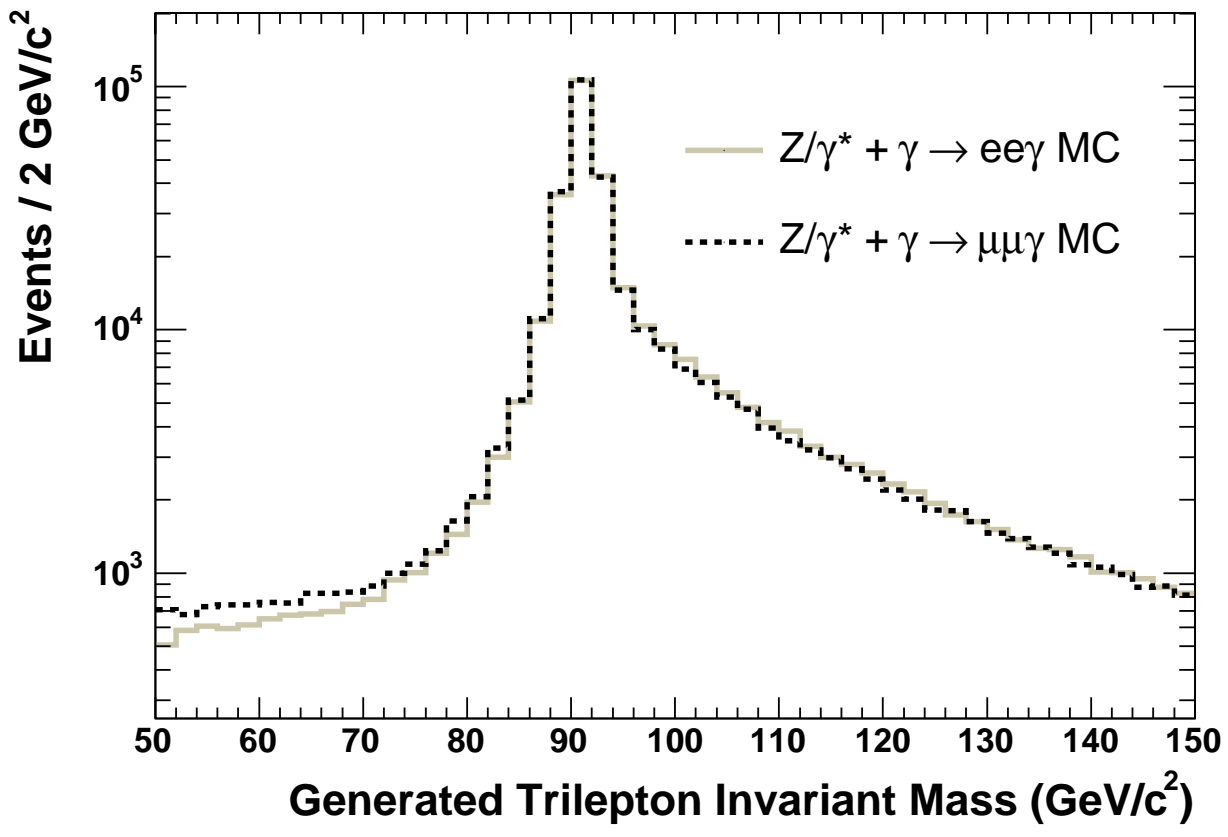

Figure A.3: Trilepton invariant mass for simulated $Z / \gamma^{*}+\gamma$ events. 


\begin{tabular}{cccc}
\hline Sample & eel & $\mu \mu \ell$ & $\ell \ell \ell$ \\
\hline$Z / \gamma^{*}+\gamma$ & $0.3 \pm 0.1$ & $0.06 \pm 0.04$ & $0.4 \pm 0.1$ \\
Total mis-ID jets & $0.2 \pm 0.1$ & $0.2 \pm 0.1$ & $0.4 \pm 0.2$ \\
$W Z / \gamma^{*}$ & $0.033 \pm 0.009$ & $0.016 \pm 0.003$ & $0.06 \pm 0.01$ \\
$Z / \gamma^{*} Z / \gamma^{*}$ & $0.015 \pm 0.008$ & $0.014 \pm 0.003$ & $0.035 \pm 0.005$ \\
\hline Total Background & $0.6 \pm 0.3$ & $0.3 \pm 0.1$ & $0.9 \pm 0.4$ \\
\hline$\not k_{p}$ SUSY $\left(\lambda_{121}\right)$ & $0.24 \pm 0.5$ & 0 & $0.45 \pm 0.07$ \\
$\not k_{p}$ SUSY $\left(\lambda_{122}\right)$ & 0 & $0.41 \pm 0.07$ & $0.52 \pm 0.07$ \\
\hline Data & 0 & 1 & 1 \\
\hline
\end{tabular}

Table A.4: Remaining events from Table A.2 after rejecting events with $76<$ $\mathrm{M}_{\ell^{+} \ell^{-} e}<106 \mathrm{GeV} / c^{2}$.

simulated events, the MC simulation must not model the data correctly. I suspected that this had something to do with low- $\mathrm{p}_{T}$ track reconstruction. Typically in simulations, there is much less "noise" in the detector corresponding to low energy particles from the proton antiproton collisions. This lack of noise could make it easier for the simulation to reconstruct tracks with large curvatures. Since photon conversion identification requires the reconstruction of both the electron and positron tracks, the MC simulation could be rejecting more conversion electrons than in the data. To determine if this is actually the case, the photon conversion identification efficiency must be measured in simulation and data. My study of this efficiency is described in Appendix B. In the end, I did find that the number of photon conversions events observed in data is underestimated by the MC depending on the $E_{T}$ of the reconstructed conversion electron. For electrons with $E_{T}=5 \mathrm{GeV}$, the MC underestimate is approximately $80 \%$ and decreases linearly to $0 \%$ at $E_{T}=30 \mathrm{GeV}$ (see Fig. B.17). As a result, the $Z / \gamma^{*}+\gamma$ prediction is 
scaled up according to the prescription detailed in the photon conversion study [71].

\section{A.6 Electron Impact Parameter Cut}

While the trilepton invariant mass requirement is effective in reducing the $Z / \gamma^{*}+\gamma$ background, it also reduces the acceptance in the $\lambda_{121}$ trilepton signal sample by over $50 \%$. Therefore, this requirement is not included in the event selection. Another requirement that was investigated as a means of reducing the $Z / \gamma^{*}+\gamma$ background was a track impact parameter cut $\left(d_{0}\right)$ on electrons. Up until this point, the $d_{0}$ cut was only applied to muons as a way to minimize the cosmic ray muon background. If the same $d_{0}$ cut is applied to electrons that is used for muons, 3 of the 8 events originally observed in data in the $\Delta \phi$ trilepton control sample are removed while leaving the background prediction nearly unchanged. With the electron $d_{0}$ cut and a more accurate prediction of $Z / \gamma^{*}+\gamma$ events in data, the final agreement in the $\Delta \phi$ control sample is $3.7 \pm 0.3$ background events compared to 6 events in data. There is a $17 \%$ probability for this background to fluctuate up to 6 or more events.

\section{A.7 Conclusions}

There are several things that I learned as a result of underestimating the $Z / \gamma^{*}+\gamma$ background and almost "discovering" supersymmetry. One thing is to double and triple check my work in the future because mistakes are inevitable, but if they are caught at an early stage then the results are the same as though they were never made in the first place. In hindsight, I could have looked in the $\Delta \phi$ trilepton control sample before examining the events in the signal sample, or even 
anticipated that the MC by itself was not sufficient to accurately determine the $Z / \gamma^{*}+\gamma$ background. If I had been more careful, I might have. When I look back, most of my mistakes were careless errors. And in each occurrence, it took more time to correct the problem than if I would have done things more carefully in the first place. I think there is tremendous pressure including internal, social, and financial to get things done within a certain amount of time. And this pressure can cause people to rush. But rushing our work is the most important thing to avoid.

I also learned that most mistakes can be corrected. It came as quite a shock, when I discovered 9 events in the $\lambda_{121}$ trilepton signal sample while expecting only $3.5 \pm 0.9$ events of background. Before I examined the events in the signal sample, I expected just to calculate the results and be finished, but instead I had to go through the process I described in this Appendix. Needless to say it was not something I wanted to do. Yet after taking a deep breath and seriously considering my options, I was able to come up with a solution.

The last important thing that I learned was not to blindly trust someone even if they have more experience. When it came time to estimate the $Z / \gamma^{*}+\gamma$ background prediction, I asked my collaborators who had performed a similar measurement before to instruct me. They advised me to make the prediction purely on the simulation. Although someone has done it before does not mean that it was done correctly. Without realizing it, I was repeating the same mistakes as others. It just happened to affect this analysis much more than in other analyses.

In conclusion, no analysis, including this one, is devoid of mistakes. Hopefully, by reflecting on them, we can avoid making the same ones in the future. 


\section{APPENDIX B}

\section{Conversion Removal Efficiency}

\section{B.1 Introduction}

Electrons originating from photon conversions represent a significant background for many lepton analyses. To reduce this background the standard tagging algorithm, developed in [72], requires two opposite sign tracks such that the $\Delta \cot \theta$ and the distance between the two tracks at closest approach $\left(D_{x y}\right)$ are less than user defined values. Both tracks must have at least one $\geq 5$ hit stereo and axial segment.

In SUSY multi-lepton searches the photon conversion background is estimated from Monte Carlo (MC), applying a scale factor for normalization to the data. In this note we report the measurement of the scale factor defined as the ratio of the photon conversion removal inefficiency $(1-\epsilon)$ in data over the inefficiency in MC. We use the inefficiency rather than the efficiency since the scale factor will be applied to the MC photon conversion events that are not removed by the conversion tagger.

The procedure for measuring the efficiency $\epsilon$ consists of the following steps:

- Select samples of photon conversion candidate events in data and MC.

- Apply the conversion tagging algorithm to the samples. 
- Calculate background subtracted efficiency as $\epsilon=\frac{N_{t c}}{N_{c c}}$, where $N_{c c}$ is the number of conversion candidates and $N_{t c}$ is the number of tagged conversion candidates.

\section{B.2 Datasets}

\section{B.2.1 Data}

We obtain the sample of photon conversion candidate events from the $8 \mathrm{GeV}$ electron calibration dataset collected via the ELECTRON_CENTRAL_8 trigger. In addition, we use the SUSY dilepton dataset, which triggers on a pair of leptons starting at $4 \mathrm{GeV}$ for electrons and $4 \mathrm{GeV} / c$ for muons.

\section{B.2.2 MC}

We use two inclusive photon samples generated with PYTHIA. One has a minimum photon $\mathrm{p}_{T}$ of $8 \mathrm{GeV}$ and the other has a minimum photon $\mathrm{p}_{T}$ of $12 \mathrm{GeV}$. For understanding the contribution of high- $\mathrm{p}_{T}$ electrons, we study a PYTHIA inclusive Drell-Yan sample with a minimum invariant mass of $5 \mathrm{GeV}$.

\section{B.3 Photon Conversion Selection}

Due to the magnetic field along the direction of the beam line, the $\mathrm{e}^{+} \mathrm{e}^{-}$from photon conversions curve in opposite directions in the transverse plane while keeping approximately the same $Z$ position measured at the CES plane. This feature allows us to predict the relative $\phi$ position of the electron with respect to the positron when the leptons reach the calorimeter. Such method of selecting the photon conversion candidates does not require any tracking cuts and therefore is 
independent of the tagging criteria and prevents the results from being biased.
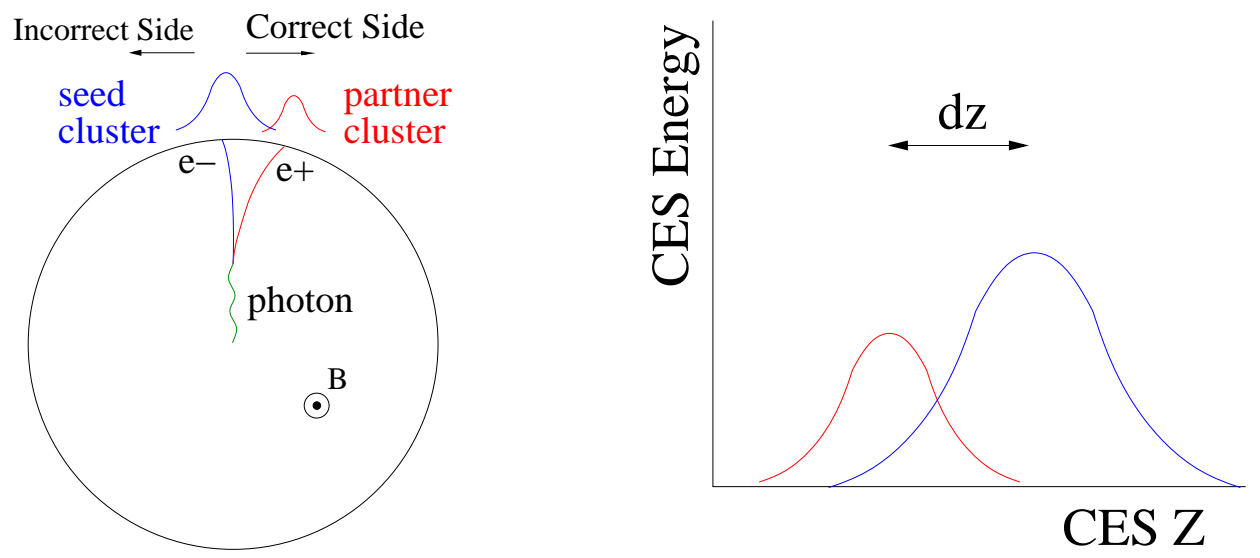

Figure B.1: Method of selecting conversion candidates and determining correct and incorrect side.

We first identify seed electrons ${ }^{1}$ with $\mathrm{E}_{T}>8 \mathrm{GeV}$ following the prescription for central loose electrons provided by the CDF Joint Physics Group [73] . Then we look for a partner CES cluster located up to $2 \phi$ wedges away from the seed wedge but not including the seed wedge. When the SUSY dilepton dataset is used, if there are more than one triggered electrons at Level 3, then they are required to be separated by 5 or more $\phi$ wedges to remove any trigger bias. If there is more than one partner cluster then the one with the highest energy is selected. Depending on the charge of the seed electron, we define the neighboring wedge as either "correct" or "incorrect" as shown in Fig. B.1. For example, if the charge of the seed electron is negative, we expect $\phi_{\text {seedEle }}>\phi_{C E S}$, whereas if it is positive, $\phi_{\text {seedEle }}<\phi_{C E S}$. If the seed electron-CES cluster pair is due to a conversion, we expect the partner cluster to be located in the correct wedges and the $\Delta Z$ between the seed electron and the CES cluster to be small. We select the conversion candidates by requiring $|\Delta Z|<20 \mathrm{~cm}$. Events where the partner

\footnotetext{
${ }^{1}$ We refer to electrons and positrons as "electrons".
} 

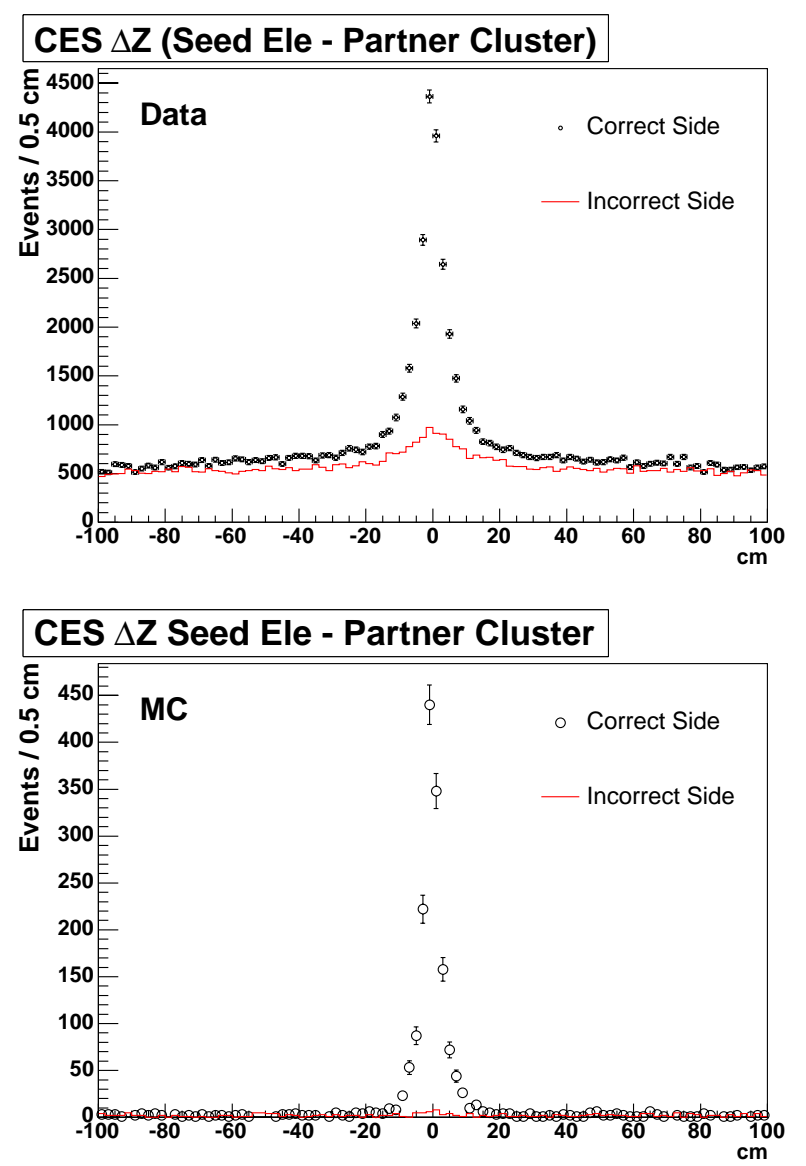

Figure B.2: $\Delta Z$ between the seed electron and the CES partner cluster for the correct (circles) and incorrect (red line) side. The top plot shows the data and the bottom shows the MC.

cluster is found in the incorrect wedges are treated as background. In Fig. B.2 we show the distribution of $\Delta Z$ in the data and MC samples: when the partner cluster is found in the correct wedge, $\Delta Z$ peaks at small values. This method of selecting conversion candidates is based on a similar study described in [74].

The energy sharing between the two electrons in photon conversions is shown in Fig. B.3. When the energy sharing is asymmetric, which is typical when we require the seed electron to be greater than $8 \mathrm{GeV}$, one of the electrons may 


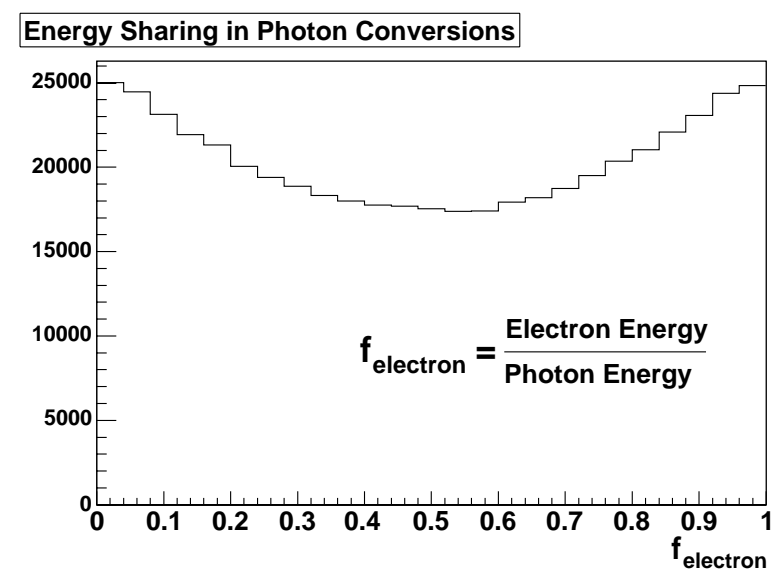

Figure B.3: Energy sharing between electron and positron in a photon conversions. A value of 0.5 corresponds to the electron and positron having equal energies.

not be reconstructed due to tracking inefficiency at low $p_{T}$. In order to include conversion candidates where one of the two tracks is not reconstructed, we use seed based CES clusters. As a result, $\epsilon$ will include the tracking efficiency.

Distributions of the conversion candidate samples can be found in Figs. B.4, B.5, B.6, and B.7.

\section{B.4 Background}

As mentioned in the previous section, a seed electron accompanied by a partner CES cluster in the correct wedge is identified as a candidate conversion if $|\Delta Z|<$ $20 \mathrm{~cm}$. In addition to photon conversions, there may be backgrounds which satisfy the conversion candidate criteria:

- electron + min bias

- electron + underlying event 

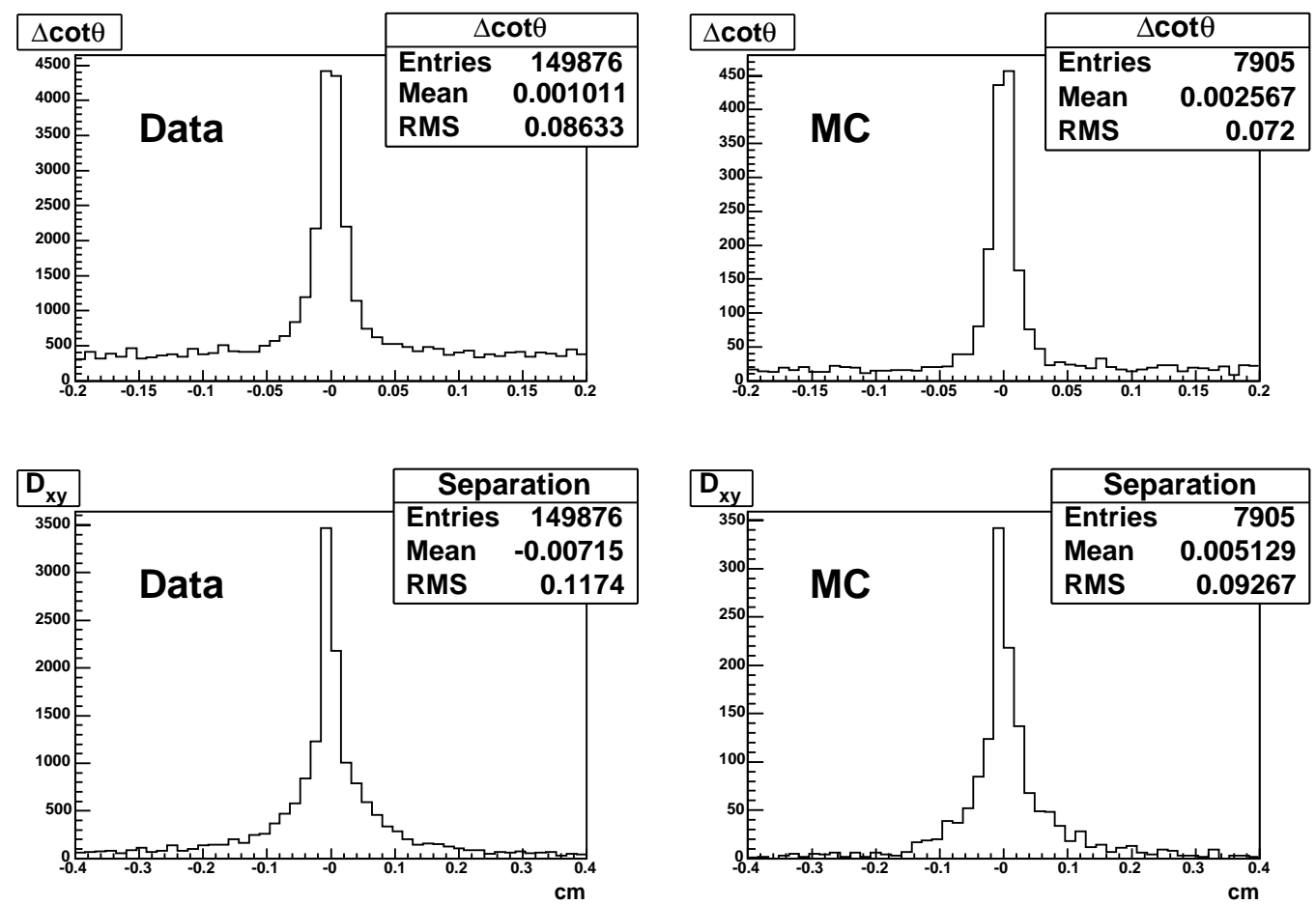

Figure B.4: Background subtracted $\Delta \cot \theta$ and $D_{x y}$ for Data and MC. Each plot is filled once for each conversion candidate that has a nearby track. For both variables the width in MC is narrower than the width in data.

- electron $+\pi^{0}$ or $K^{ \pm}$

- $e+\gamma$ ("brem")

- $e+\left(\gamma \rightarrow e^{+} e^{-}\right)$("tridents")

- jets (e.g. $\left.\pi^{ \pm}+\left(\pi^{0} \rightarrow \gamma \gamma\right)\right)$

The background is estimated by the number of events in the incorrect wedge. This method will take care of the first three backgrounds which are expected to be flat in $\Delta Z$, and to populate the correct and incorrect wedges with equal probability. For events where an electron radiates a photon, there may be more 

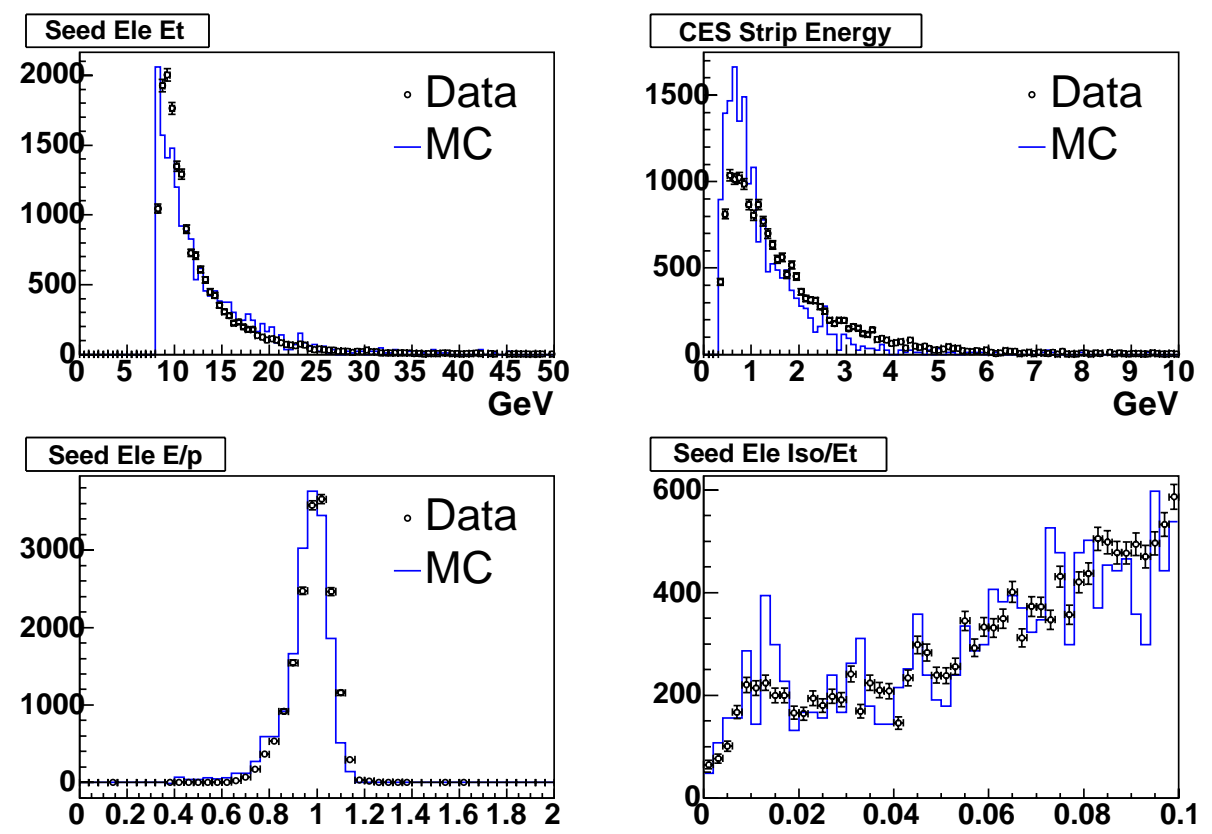

Figure B.5: Background subtracted Data and MC distributions of photon conversion candidates: seed electron transverse energy (top left), CES partner cluster energy (top right), seed electron $E / p$ (bottom left), and seed electron fractional isolation (bottom right). The $\mathrm{MC}$ is normalized to the data by the number of events. The plots are filled once per conversion candidate.

events in either the correct or incorrect side. To determine how this may impact the conversion removal efficiency, we first describe how we expect the electron + photon events to be identified (correct or incorrect side, tagged as a conversion or untagged) and then we measure these numbers in DY MC:

- $e+\gamma$ ("brem") - In this case the electron curves while the photon propagates along the original electron direction and creates a CES cluster on the correct side as shown in Fig. B.8. For most events we expect the electron to retain most of its original energy and therefore the photon should be relatively close to the electron. Since there is no track associated with the photon, 


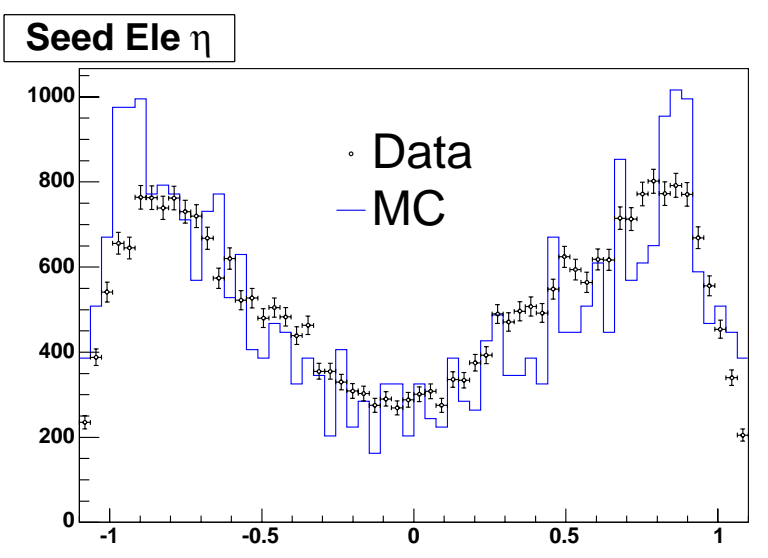

Figure B.6: Seed electron $\eta$ for data and MC. Plot is filled once for each candidate conversion event.

such an event is not expected to be identified as a conversion.

- $e+\left(\gamma \rightarrow e^{+} e^{-}\right)$("tridents") - When the photon converts, either conversion electron may create a CES cluster. Typical trident events are shown in Fig. B.9. If only the opposite sign electron is reconstructed then we expect the partner on the correct side and the seed to be tagged as a conversion. Otherwise if only the same sign electron is selected then the partner cluster could be on either side but the event should be untagged. In the scenario when both tracks are reconstructed we expect the event to be tagged regardless of which cluster is chosen.

To reduce the electron + photon background, we apply cuts to reject electrons that do not originate from photon conversions. We place a cut on the transverse missing energy $\left(\mathscr{E}_{T}<15 \mathrm{GeV}\right)$ to remove $W$ events and a cut on the dielectron invariant mass cut $\left(50<m_{e e}<106 \mathrm{GeV}\right)$ to reject on-shell $Z$ events. On top of the kinematic cuts, we also require the $E / p$ of the seed electron to be less than 1.1 to reduce the electron background. If the electron radiates a photon, 


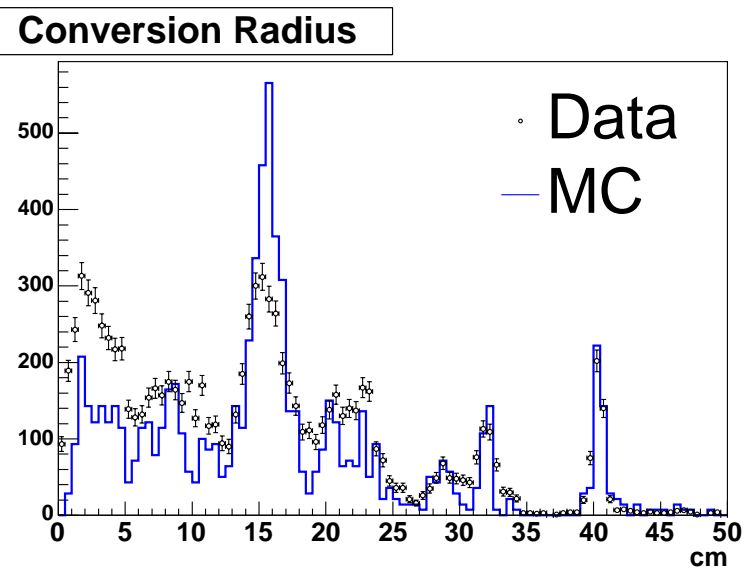

Figure B.7: Background subtracted radius of conversion for Data and MC. Plot is filled once for each seed electron that is tagged as a conversion. MC is normalized to data based on the number of events that convert at the COT endplate.

the track $p_{T}$ decreases by the energy given off to the photon while the $E_{T}$ does not decrease since the calorimeter can not distinguish between the electron and the photon, resulting in an $E / p>1$.

To estimate the electron + photon background contribution, brem and trident events are selected by looking at the generation and simulation information in DY MC. First there must be a seed electron matching one at the generator level. Second a photon with $\mathrm{p}_{T}>0.5 \mathrm{GeV}$ must originate from the electron at either generator or simulator level. If no such photon is found, the seed electron is rejected. And third we check to see if the photon converts into an electronpositron pair in the simulation. If the photon converts, the electron is selected as a trident, otherwise it is classified as a brem electron. For each sample, we see how often there is a partner cluster on the correct and incorrect sides and how often the electron is tagged as a conversion as shown in Table B.1. The $\Delta Z$ distributions for the correct and incorrect side are plotted in Fig. B.10. 


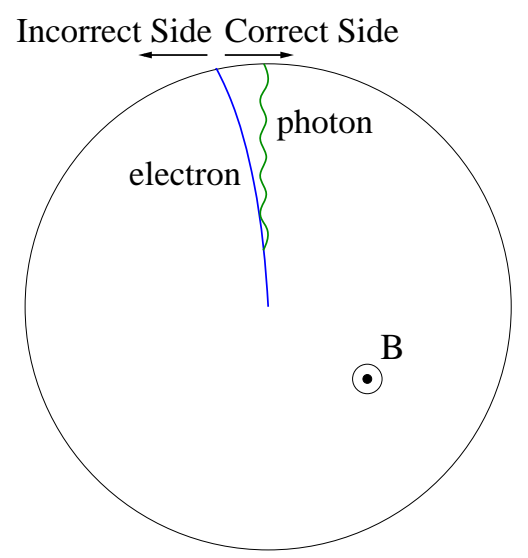

Figure B.8: A "brem" event where an electron radiates a photon. The photon will be on the correct side.
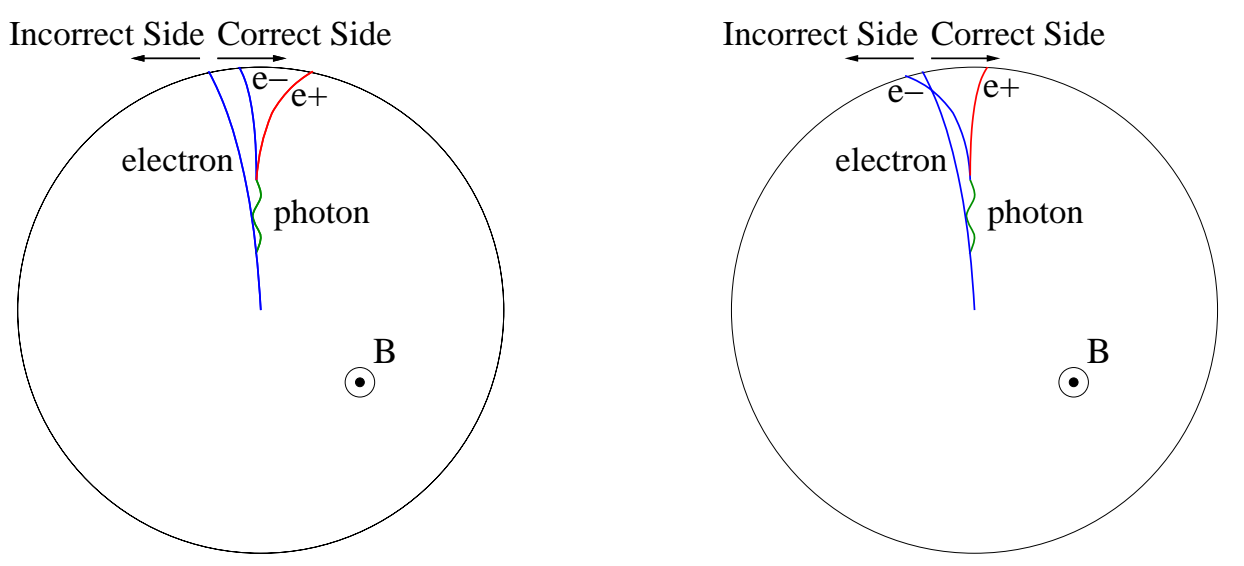

Figure B.9: Two "trident" events where an electron radiates a photon that converts. 

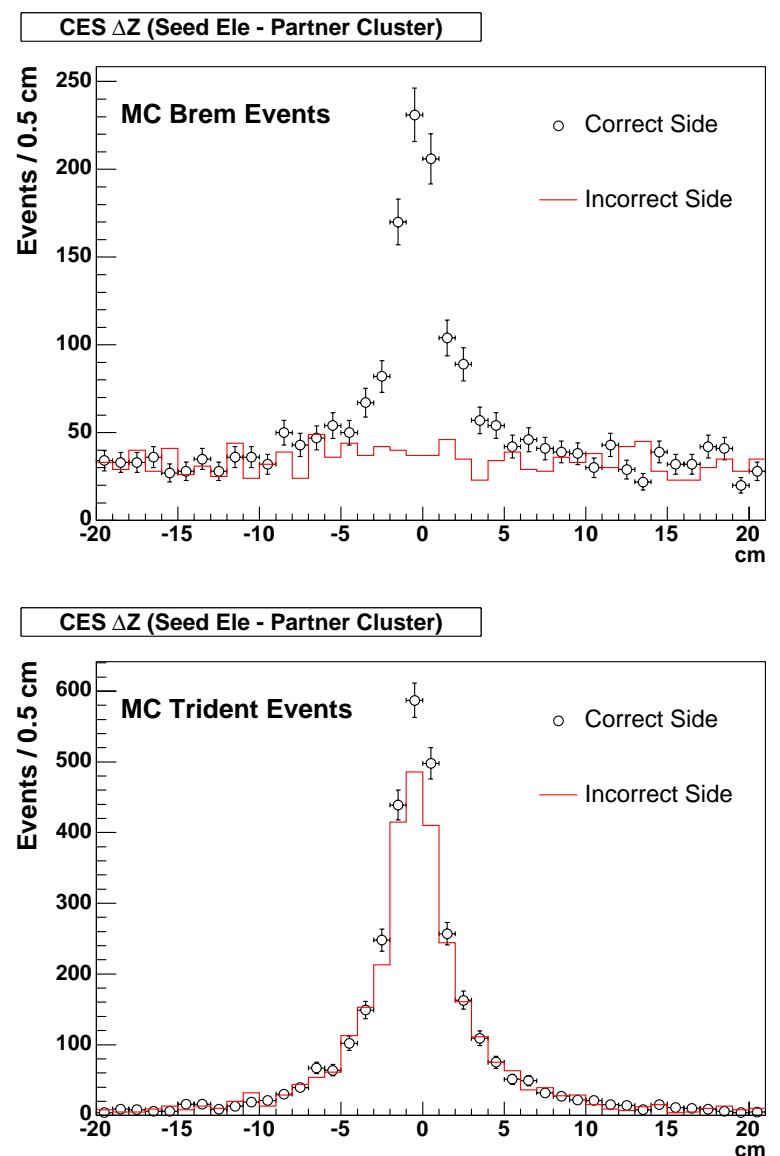

Figure B.10: $\Delta Z$ between the seed electron and the CES partner cluster for the correct (circles) and incorrect (red line) side. The top plot shows MC Brem. events and the bottom shows MC trident. 


\begin{tabular}{l|cc} 
& Brem Electrons & Trident Electrons \\
\hline Correct Side \& Tagged & 21 & 2168 \\
Correct Side \& Untagged & 2177 & 1080 \\
Incorrect Side \& Tagged & 4 & 883 \\
Incorrect Side \& Untagged & 1359 & 2115
\end{tabular}

Table B.1: Number of brem and trident events in DY MC that have a seed electron + CES partner cluster. We split the events into correct (conversion candidate) and incorrect side (background candidate) depending on the relative position of the partner cluster and whether the seed electron is tagged as a conversion. A conversion candidate (or background) is considered tagged if it passes the conversion removal cuts.

In Fig. B.10 we see that the trident events have a peak at $\Delta Z=0$ for both the correct and incorrect sides. The overall number of events is the same for the correct and incorrect sides but only when we consider tagged and untagged events together as shown in Table B.1. Therefore having a significant number of trident events will skew the conversion removal efficiency. We can get a rough estimate of how many trident events make it into our signal sample by looking for a peak at $\Delta Z=0$ in the incorrect wedge in data. When we fit the small peak in the incorrect side of Fig. B.2 we obtain $\sigma_{\text {data }}=10.2 \mathrm{~cm}$. This is larger than what we would expect from MC trident events (Fig. B.10) where the same fit returns $\sigma_{M C}=2.3$ $\mathrm{cm}$. We do not believe that this is due to extreme smearing in the data since we do not see the same behavior in the $\Delta Z$ peak for tagged conversion candidates: $\sigma_{M C}=2.4 \approx \sigma_{\text {data }}=2.7 \mathrm{~cm}$. As an additional cross check, we plot $\Delta Z$ for events where the CES partner appears in both the unbiased and the track based CES collections. This sample should have significantly less background since we are 
requiring a second track making the trident background more prominent. In Fig. B.11 we see that the incorrect side has no peak at $\Delta Z=0$. We conclude that the trident contribution is negligible.

For the brem background, there is no way to estimate how large it is by looking at $\Delta Z$ since the distribution looks identical to the signal. In most of the events, the radiated photon occupies the same wedge as the seed electron. Since we do not accept this configuration, this background is suppressed. Also the number of brem events is of the same order as trident events as in Table B.1, so we conclude that the brem background is negligible.

We ran over the Pythia dijet sample to select conversion candidates where a jet fakes a seed electron as well as a partner cluster. None were selected, indicating that the luminosity of the sample is insufficient to determine the jet background fraction. Regardless, we expect jets to populate the correct and incorrect side with equal probability and therefore will be removed when we apply the background subtraction. Since jets are localized, they could account for the $\Delta Z$ peak on the incorrect side (Fig. B.2).

\section{B.4.1 Background Subtraction}

Background is removed by subtracting the incorrect side from the correct side. We expect that there should be very little signal with $\Delta Z>20 \mathrm{~cm}$ and therefore the tails of the $\Delta Z$ distribution should be identical for the incorrect and correct sides. In Fig. B.2, we see that this is not the case and therefore there must be additional background that is not being taken into account in the incorrect side. To remove additional background, we scale up the incorrect side so that the tails of the $\Delta Z$ distribution agree before it is subtracted from the correct side. 


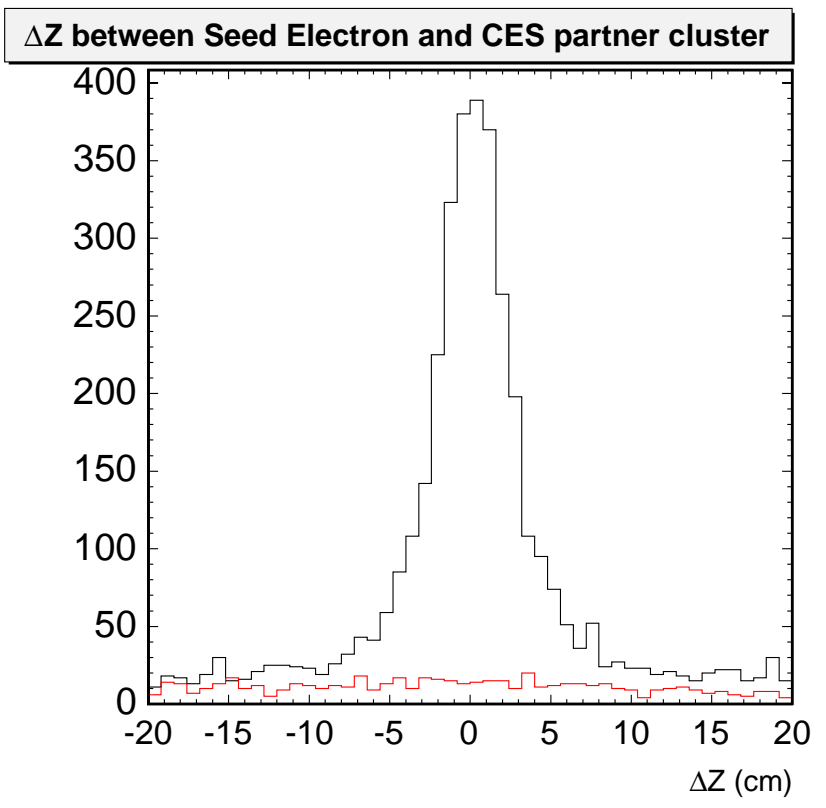

Figure B.11: $\Delta Z$ for events where the partner cluster has both an unbiased CES cluster and a track based CES cluster. The black line displays the correct side and the red line displays the incorrect side. 


\begin{tabular}{cc|cc}
$\Delta \cot \theta$ & $\mathrm{D}_{x y}$ & $\epsilon_{\text {Data }}$ & $\epsilon_{M C}$ \\
\hline 0.02 & 0.1 & $64.6 \pm 0.4 \%$ & $72.6 \pm 1.2 \%$ \\
0.04 & 0.2 & $83.8 \pm 0.3 \%$ & $88.2 \pm 0.8 \%$
\end{tabular}

Table B.2: Conversion removal efficiency in Data and MC.

\section{B.5 Efficiency Measurement}

Once the candidate events are selected in both data and MC, the tagging algorithm is applied and the efficiency is measured as:

$$
\epsilon=\frac{N_{t}^{\text {signal }}-N_{t}^{\text {background }}}{N_{c c}^{\text {signal }}-N_{c c}^{\text {bockground }}}
$$

where $N_{c c}$ is the number of conversion candidates and $N_{t c}$ is the number of tagged conversion candidates. The overall efficiency is shown in Table B.2, while in Fig. B.12 the efficiency is shown as a function of seed electron $\mathrm{E}_{T}$.

The conversion candidate sample (CES sample) that we used to measure the efficiency is not inclusive since events where the partner electron does not create a CES cluster within two wedges from the seed electron are not selected. There are several reasons why the cluster is not found using the procedure described in Section B.3:

- Electron $\mathrm{p}_{T}$ is small so that it reaches a $\phi$ wedge which is more than two wedges from the seed ( $a$ in Fig. B.13).

- Electron $\mathrm{p}_{T}$ is so small that it never reaches the calorimeter( $b$ in Fig. B.13).

- Electron $\mathrm{p}_{T}$ is large enough so that the partner cluster is in the seed wedge ( $c$ in Fig. B.13). 


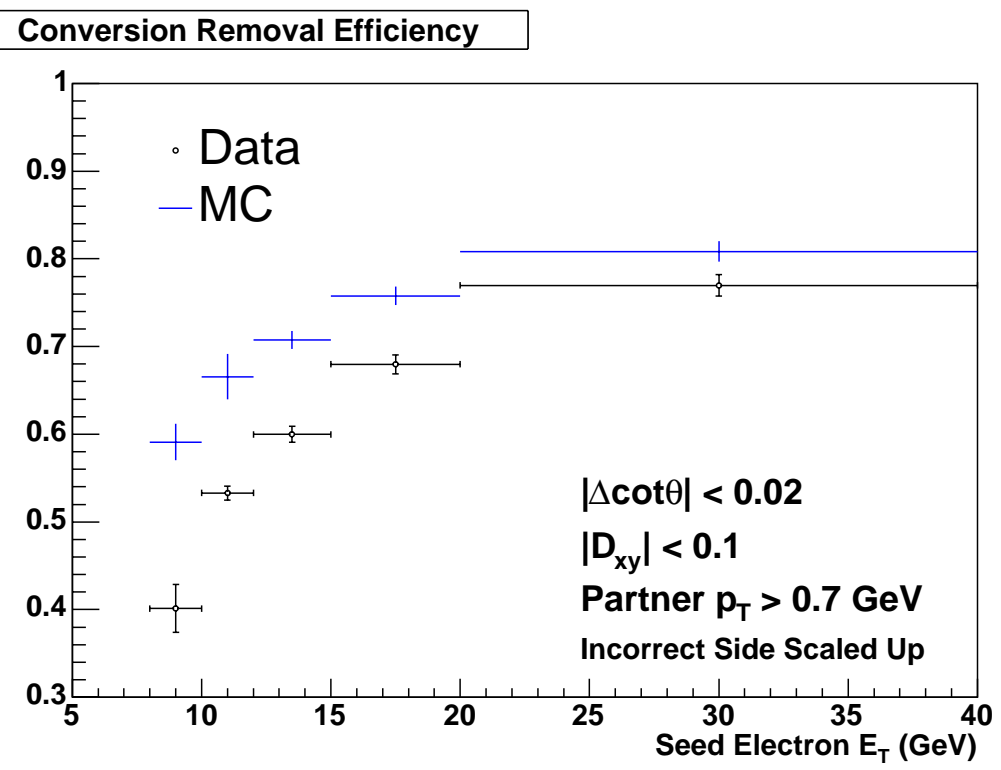

Figure B.12: Conversion removal efficiency for data and $\mathrm{MC}$ as a function of the seed electron $\mathrm{E}_{T}$.

- Electron transverses a region which is not fiducial to the CES or there is a dead channel.

To determine the efficiency of these events in the MC, we select all conversions at simulation level that are matched to a seed electron, removing events that would be classified as CES sample. We refer to this new sample as the very low $\mathrm{p}_{T}$ sample. The $\mathrm{p}_{T}$ of the two electrons coming from the photon conversion is plotted in Fig. B.14 for this sample. Applying the conversion filter, we measure $\epsilon_{M C}=36.4 \pm 0.6 \%$.

The tagging efficiency of the very low $\mathrm{p}_{T}$ sample is lower than the efficiency measured in the CES sample. This is most likely due to lower tracking reconstruction for low $\mathrm{p}_{T}$ tracks since there must be at least two tracks for an electron to be tagged as a conversion. In the data, we can not measure the efficiency for the events that do not make it into the CES sample therefore we proceed as 


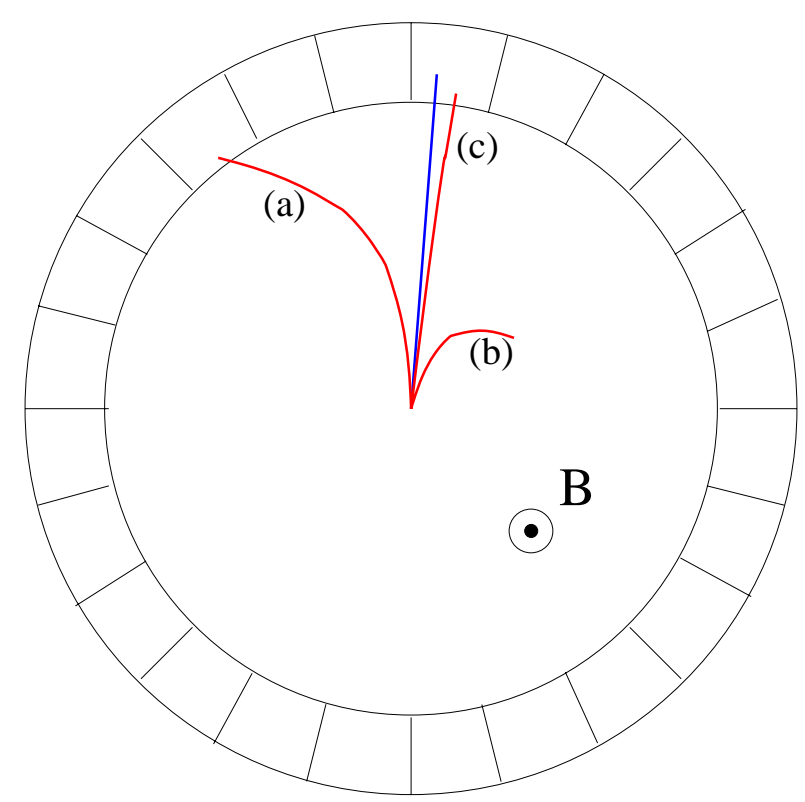

Figure B.13: CES clusters for photon conversions belonging to the very low $\mathrm{p}_{T}$ sample: the blue line represents the seed electron, the red lines represent different scenario for the CES partner cluster.

follows:

- We measure the efficiency for the very low $\mathrm{p}_{T}$ sample as a function of the partner track minimum $\mathrm{p}_{T}$. We choose a $\mathrm{p}_{T}$ threshold such that the efficiency in the very low $\mathrm{p}_{T}$ sample equals the efficiency in the CES sample without $\mathrm{p}_{T}$ requirement.

- We restrict the tagging algorithm such that it only uses tracks above the determined $\mathrm{p}_{T}$ threshold.

First the reconstructed seed electron is matched to a photon conversion identified at simulation level. Second we select the reconstructed track with the minimum distance $\left(d=\sqrt{(5 \times \Delta \cot \theta)^{2}+D_{x y}^{2}}\right)$ to the simulated partner track. 


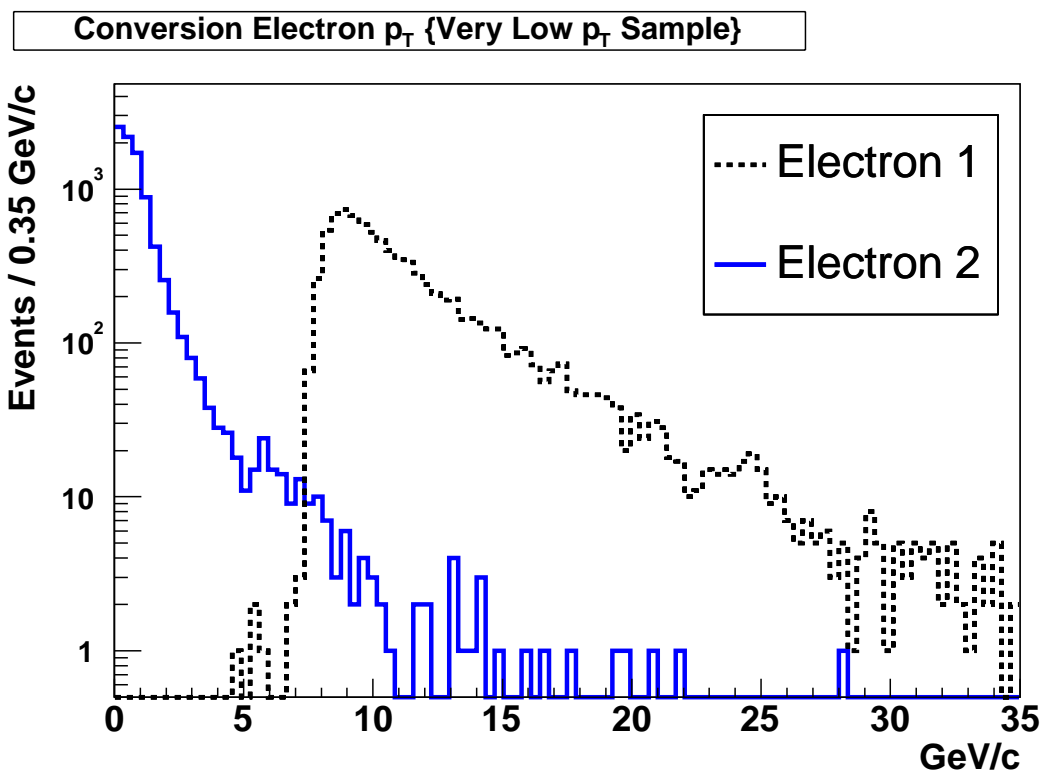

Figure B.14: Conversion electron $\mathrm{p}_{T}$ for the very low $\mathrm{p}_{T}$ sample.

\begin{tabular}{cc|cc}
$\Delta \cot \theta$ & $\mathrm{D}_{x y}$ & $\epsilon_{\text {Data }}$ & $\epsilon_{M C}$ \\
\hline 0.02 & 0.1 & $59.4 \pm 0.4 \%$ & $66.9 \pm 1.2 \%$ \\
0.04 & 0.2 & $72.2 \pm 0.4 \%$ & $79.7 \pm 1.0 \%$
\end{tabular}

Table B.3: Conversion removal efficiency in Data and $\mathrm{MC}$ when a min $\mathrm{p}_{T}$ cut is applied.

If the simulated $\mathrm{p}_{T}$ is small than there may not be a reconstructed track and the event is not considered in the efficiency. The efficiency as a function of the minimum reconstructed $\mathrm{p}_{T}$ is shown in Fig. B.15. Based on this plot we obtain the $\mathrm{p}_{T}$ threshold of $0.7 \mathrm{GeV}$.

Placing a cut of $\mathrm{p}_{T}>0.7 \mathrm{GeV}$ on the partner track on the very low $\mathrm{p}_{T}$, we obtain the results in Table B.3.

We expect the reconstruction efficiency of very low $\mathrm{p}_{T}$ electrons to be worse for data than it is for MC due to more noise in the tracking volume and calorimeter. 


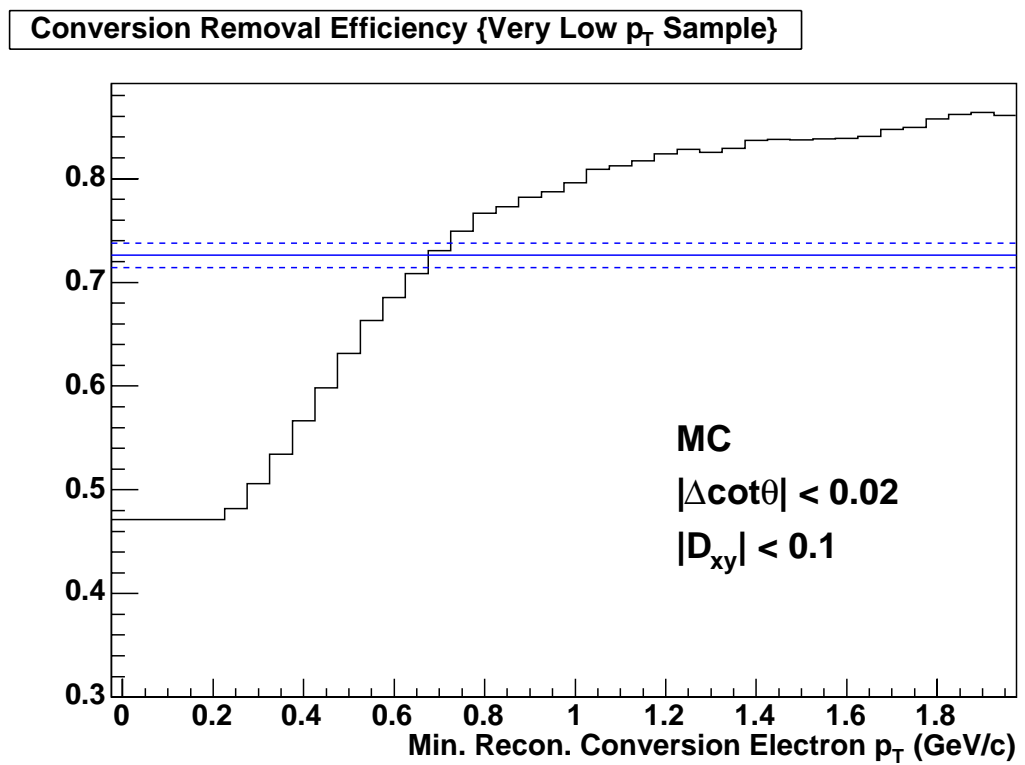

Figure B.15: Efficiency for the very low $\mathrm{p}_{T}$ sample as a function of the partner track minimum $\mathrm{p}_{T}$.

This means that the conversion filter will be able to tag and reject more of these events in MC than in data. By discarding tracks below a certain $\mathrm{p}_{T}$, such a discrepancy between data and $\mathrm{MC}$ will no longer be a factor in the conversion removal efficiency. As a result the conversion background estimated from MC will increase but this expectation should be in better agreement with the observed number in data.

There are three sources of conversion removal inefficiency: track reconstruction, $\Delta \cot \theta$ cut, and $D_{x y}$ cut. To estimate the contribution of each source to the total inefficiency we select events requiring a track based CES cluster for each selected unbiased CES partner cluster. In this way we are certain that each candidate cluster has a track associated to it and we can measure the efficiency excluding the tracking reconstruction. We then apply the other cuts sequentially on the track based sample and the results are listed in Table B.4. Note that track 


\begin{tabular}{l|cc} 
Source & Data & MC \\
\hline$\Delta \cot \theta$ & $76.6 \pm 0.4 \%$ & $80.6 \pm 1.0 \%$ \\
$D_{x y}$ & $78.4 \pm 0.4 \%$ & $84.5 \pm 1.0 \%$
\end{tabular}

Table B.4: Efficiency of each conversion requirement. Cuts are applied sequentially.

based CES clusters have a minimum track $\mathrm{p}_{T}$ requirement of $1 \mathrm{GeV}$ therefore the inefficiency that we attribute to the tracking reconstruction is underestimated.

\section{B.6 Scale Factor Measurement and Implementation}

The scale factor is calculated as the ratio of the inefficiencies in data and MC for each $E_{T}$ bin. The scale factor as a function of seed electron $E_{T}$ is shown in Fig. B.16. We see that the scale factor decreases with $\mathrm{E}_{T}$, signifying that the $\mathrm{MC}$ models the data better at higher values of electron energy. For electron $\mathrm{E}_{T}$ where the fit is $<1$, we use a scale factor $=1$ corresponding to perfect agreement between data and MC.

This scale factor must be applied to every electron originating from a photon that is not removed by the conversion tagger. For example, if we are estimating the trilepton background in $\mathrm{Z}+\gamma \rightarrow \mu \mu+\gamma \mathrm{MC}$, then we accept events with two muons and one electron where the electron is matched to a generated photon. The total number of events is multiplied by the scale factor to get the final prediction in data. 


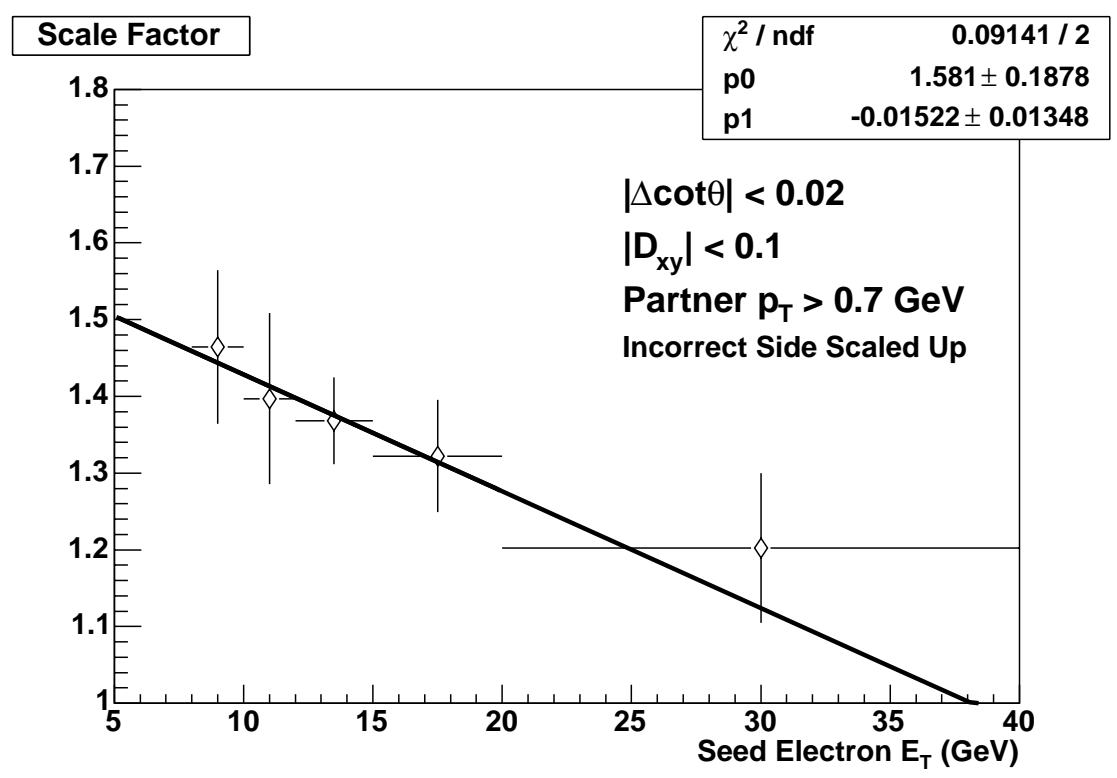

Figure B.16: Conversion removal scale factor as a function of the seed electron $\mathrm{E}_{T}$. Only the first $4 \mathrm{E}_{T}$ bins are used in the fit. The last bin is not used since the mean $\mathrm{E}_{T}=25 \mathrm{GeV}$ does not equal the bin center and would bias the fit.

\section{B.7 Uncertainty}

The systematic uncertainties affecting the conversion removal efficiency are:

- Choice of partner track $p_{T}$ threshold.

- Choice of $\Delta Z$ cut.

- Background subtraction.

- CES cluster reconstruction efficiency at low energies.

- Eta dependence. 


\section{B.7.1 $p_{T}$ Threshold}

We estimate the systematic uncertainty due to the choice of the $\mathrm{p}_{T}$ threshold $=$ $0.7 \mathrm{GeV}$ by measuring the scale factor for minimum $\mathrm{p}_{T}$ values of $0.6 \mathrm{GeV}$ and 0.8 $\mathrm{GeV}$. The uncertainty per $\mathrm{E}_{T}$ bin is defined as: $\delta_{\text {scalefactor }}=\left(S F^{0.6}-S F^{0.8}\right) /(2 *$ $\left.S F^{0.7}\right)$. Averaging over all the $\mathrm{E}_{T}$ bins we get: $\delta_{\text {scalefactor }}=12.7 \%$.

\section{B.7.2 $\Delta Z$ Cut}

To estimate the uncertainty due to the $\Delta Z$ cut, we measure the scale factor for values above and below the nominal value of $20 \mathrm{~cm}$. The uncertainty is calculated as: $\delta_{\text {scalefactor }}=\left(S F^{25}-S F^{15}\right) /\left(2 * S F^{20}\right)=3.3 \%$.

\section{B.7.3 Background Subtraction}

In Section B.4.1, we described how the incorrect side is scaled up to account for additional background. This represents an uncertainty in our understanding of the background. To account for this, we vary the scale up factor by $10 \%$ and observe how the conversion removal scale factor changes. $\delta_{\text {scalefactor }}=$ $\left(S F^{\text {scale } * 0.9}-S F^{\text {scale } * 1.1}\right) /\left(2 * S F^{\text {scale } * 1.0}\right)=19.6 \%$.

\section{B.7.4 CES Reconstruction Efficiency}

We estimate the systematic uncertainty due to the CES reconstruction efficiency by comparing the scale factors for photon candidates with CES cluster E $>0.7$ $\mathrm{GeV}$ and $\mathrm{E}<0.7 \mathrm{GeV}$. We obtain: $\delta_{S F}=S F^{E>0.7}-S F^{E<0.7}=5.4 \%$. 


\section{B.7.5 Eta Dependence}

We expect the scale factor to be the same for conversions in the east and west

sides. Any difference is taken as a systematic error: $\delta_{\text {scalefactor }}=\left(S F^{\text {East }}-\right.$ $\left.S F^{\text {West }}\right) /\left(2 * S F^{\text {Nominal }}\right)=15.7 \% . S F^{\text {Nominal }}$ is the scale factor measured in Section B.6.

\section{B.7.6 Total Uncertainty}

We obtain the total systematic uncertainty by combining all of the previous measurements assuming that they are uncorrelated: $\delta_{\text {scalefactor }}=28.8 \%$.

\section{B.8 Conclusions}

We have measured the photon conversion removal scale factor for generation 5 using $\Delta \cot \theta<0.02$ and $D_{x y}<0.1 \mathrm{~cm}: \mathrm{SF}=\operatorname{Max}\left[1,\left(1.581-0.0152^{*} \mathrm{E}_{T}\right)\right]$.

With the looser conversion cuts, $\Delta \cot \theta<0.04$ and $D_{x y}<0.2 \mathrm{~cm}$, the $\mathrm{SF}=$ $\operatorname{Max}\left[1,\left(1.979-0.0320^{*} \mathrm{E}_{T}\right)\right]$. The scale factor is shown in Fig. B.17.

In both cases, only conversion tracks with $\mathrm{p}_{T}>0.7 \mathrm{GeV}$ are considered. The total uncertainty on the conversion removal scale factor $=28.8 \%$. 


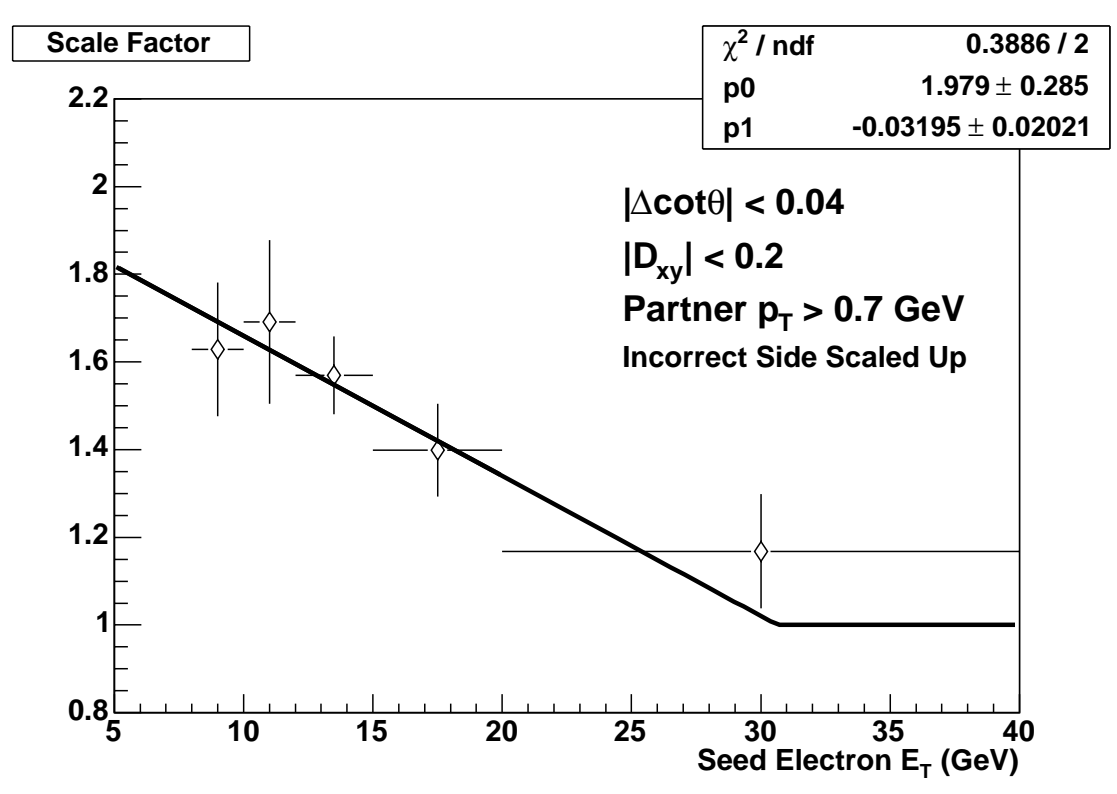

Figure B.17: Conversion removal scale factor as a function of the seed electron $\mathrm{E}_{T}$. Only the first $4 \mathrm{E}_{T}$ bins are used in the fit. The last bin is not used since the mean $\mathrm{E}_{T}=25 \mathrm{GeV}$ does not equal the bin center and would bias the fit. 


\section{REFERENCES}

[1] B. Watterson, There's Treasure Everywhere!, Andrews and McMeel, Kansas City, 1996.

[2] G. Gamow, Thirty Years That Shook Physics: The Story of Quantum Theory, World Scientific, New Jersey, 1966.

[3] The Particle Adventure: http://particleadventure.org/particleadventure/.

[4] M. Veltman, Facts and Mysteries in Elementary Particle Physics, Anchor Books, New York, 2003.

[5] D. Griffiths, Introduction to Elementary Particles, John Wiley \& Sons, New York, 1987.

[6] P.D.B. Collins and A.D. Martin, Hadron Interactions, Adam Hilger Ltd, Bristol, Great Britain, 1984.

[7] T. Sjöstrand, L. Lönnblad, and S. Mrenna, hep-ph/0108264, 2001.

[8] M. A. Tavel's English translation of Emmy Noether's 1918 publication, physics/0503066.

[9] V. V. Belokurov and D. V. Shirkov, The Theory of Particle Interactions, American Institute of Physics, New York, 1991.

[10] T.D. Lee and C.N. Yang, Phys. Rev. 104:254, 1956; C. S. Wu et. al., ibid., 105:1413, 1957.

[11] V. Barger, D. Marfatia, and A. Tregre, Phys. Rev. lett. B595:55-59, 2004.

[12] A. Abulencia et. al., Phys. Rev. Lett. 96:022004, 2006.

[13] P.W. Higgs, Phys. Rev. Lett. 13:508, 1964.

[14] P.W. Higgs, Phys. Rev. 145:1156, 1966.

[15] S. L. Glashow, Nucl. Phys. 22:579, 1961; S. Weinberg, Phys. Rev. Lett. 19: 1264, 1967; A. Salam, Weak and Electromagnetic Interactions, Proceedings of the Nobel Symposium held at Lerum, Sweden, 1968.

[16] S. Pokorski, hep-ph/0502132, 2005.

[17] http://superstringtheory.com/. 
[18] http://map.gsfc.nasa.gov/.

[19] G. Gamow, Phys. Rev. 70:572, 1946; R. A. Alpher et. al., Phys. Rev. 73:803, 1948.

[20] R.A. Alpher and R. C. Herman, Phys. Rev. 74:1737, 1948; R.A. Alpher and R. C. Herman, ibid., 75:1089, 1949.

[21] S.P. Martin, hep-ph/9709356, 1999.

[22] L. Girardello and M. Grisaru, Nucl. Phys. B194:65, 1982.

[23] W.-M. Yao et. al. (Particle Data Group), J. Phys. G 33:1, 2006.

[24] H.P. Nilles, Phys. Rep. 110:1, 1984.

[25] R. Barbier et. al., Phys. Rep. 420:1, 2005.

[26] W. Beenakker et. al., Phys. Rev. Lett. 83:3780-3783, 1999.

[27] A. Köngeter, Fermilab-Thesis-1999-33.

[28] V. M. Abazov et. al., (DØ Collaboration), Phys. Lett. B638:441, 2006.

[29] F. Abe et. al. (CDF Collaboration), Phys. Rev. Lett. 76:4307, 1996; F. Abe et al. (CDF Collaboration), ibid., 80:5275, 1998; S. Abachi et. al. (DØ Collaboration), ibid., 76:2228, 1996; B. Abbott et. al. (DØ Collaboration), ibid., 80:1591, 1998; V.M. Abazov et. al. (DØ Collaboration), ibid., 95:151805, 2005.

[30] H. Baer et. al., hep-ph/0312045, 2003.

[31] C.S. Aulakh, A. Melfo, and G. Senjanovic, Phys. Rev. D57:4174, 1998; Z. Chacko and R. N. Mohapatra, ibid., D58:015003, 1998.

[32] V. Barger, P. Langacker, and H.-S. Lee, hep-ph/0508027, 2005.

[33] D. Acosta et. al., Nucl. Instrum. Meth. A461:540-544, 2001.

[34] D. Acosta et. al., Nucl. Instrum. Meth. A494:57-62, 2002.

[35] P. Gatti, Fermilab-Thesis-2001-23.

[36] A. Sill, Nucl. Instrum. Meth. A447:1-8, 2000.

[37] T. Affolder et. al., Nucl. Instrum. Meth. A526:249-299, 2004.

[38] L. Balka et. al., Nucl. Instrum. Meth. A267:272-279, 1998. 
[39] S. Bertolucci et. al., Nucl. Instrum. Meth. A267:301-314, 1998.

[40] M. Albrow et. al., Nucl. Instrum. Meth. A480:524-545, 2002.

[41] R. Blair et. al. (CDF Collaboration), Fermilab Report No. FERMILABPub-96-390-E, 1996, Section 9.

[42] G. Apollinari et. al., Nucl. Instrum. Meth. A412:515-526, 1998.

[43] A. Artikov et. al., Nucl. Instrum. Meth. A538:358-371, 2005.

[44] T. Sjöstrand et. al., Computer Physics Commun. 135:238, 2001.

[45] S. Agostinelli et. al., Nucl. Instrum. Meth. A506:250-303, 2003.

[46] G. Grindhammer and S. Peters, hep-ex/0001020, 1993.

[47] C. Hill, J. Incandela, and C. Mills, CDF/DOC/ELECTRON/CDFR/7309.

[48] M. Griffiths, B. Heinemann, and G. Manca, CDF/ANAL/EXOTIC/CDFR/7233.

[49] S. Dube, J. Zhou, and S. Somalwar, CDF/DOC/EXOTIC/CDFR/7379.

[50] A. Attal, CDF/ANAL/EXOTIC/CDFR/7345.

[51] V. Martin, CDF/DOC/MUON/7367.

[52] D. Bortoletto et. al., CDF/ANAL/EXOTIC/CDFR/7197.

[53] M. Gold and V. Rekovic, CDF/ANAL/EXOTIC/CDFR/7210.

[54] R. Blair et. al. (CDF Collaboration), Fermilab Report No. FERMILABPub-96-390-E, 1996, Section 12.

[55] R.G. Wagner, CDF/DOC/ELECTRON/CDFR/6249, 2003.

[56] T. LeCompte, CDF/ANAL/MUON/CDFR/6114, 2002.

[57] The DØ Collaboration, "Search for Supersymmetry with R-parity Violation in the eel Final State", Aug. 4, 2004, DØ Note 4522-CONF.

[58] The DØ Collaboration, "A Search for R-parity Violating Supersymmetry in the $\mu \mu \ell(\ell=e, \mu)$ Channel", Aug. 4, 2004, DØ Note 4490-CONF.

[59] M. Handford, Where's Waldo?, Candlewick Press, Massachusetts, 1997.

[60] S. Drell and T.M. Yan, Phys. Rev. Lett. 25:316-320, 1970. 
[61] G. Manca, M Griffiths, and B. Heinemann, CDF/ANAL/EXOTIC/CDFR/7499, 2005.

[62] U. Baur and E.L. Berger, Phys. Rev. D41:1476-1488, 1990; U. Baur and E.L. Berger, Phys. Rev. D47:4889-4904, 1993.

[63] F. Maltoni and T. Stelzer, hep-ph/0208156; T. Stelzer and W.F. Long, Phys. Commun. 81:357, 1994.

[64] M. Cacciari et. al. JHEP 404:68, 2004; N. Kidonakis and R. Vogt, Phys. Rev. D68:114014, 2003.

[65] J.R. Taylor, An Introduction to Error Analysis, University Science Books, Sausalito, CA, 1982; G. Cowan, Statistical Data Analysis, Oxford University Press, Oxford, 1998.

[66] J. Pumplin et. al. hep-ph/0201195, 2002; http://www.phys.psu.edu/〜 cteq/.

[67] A.D. Martin, R.G. Roberts, and W.J. Stirling, Phys. Rev. D50:6734-6752, 1994; http://durpdg.dur.ac.uk/hepdata/mrs.html.

[68] D. Bortoletto, A. Canepa, and E. Lytken, CDF/ANAL/EXOTIC/CDFR/8114, 2006.

[69] J. Heinrich et. al., physics/0409129, 2004.

[70] Aleph Collaboration, Eur. Phys. J. C31:1, 2003; L3 Collaboration, Phys. Lett. B524:65, 2002; R. Barbier et. al. (Delphi Collaboration), DELPHI 2002-036 CONF 570, Contributed Paper for ICHEP 2002.

[71] A. Attal, A. Canepa, CDF/ANAL/ELECTRON/CDFR/8073, 2006.

[72] D. Gerdes, CDF/ANAL/TOP/CDFR/2903, 1994.

[73] http://www-cdf.fnal.gov/internal/physics/joint_physics/instructions/electron_cuts.html.

[74] H. Kobayashi, K. Yamamoto, and Y. Seiya, CDF/ANAL/EXOTIC/CDFR/7261, 2004. 